\author{
Universidade de São Paulo \\ Instituto de Física
}

\title{
Estudo do espectro contínuo de raios-X induzido pela passagem de prótons de alguns $\mathrm{MeV}$ na matéria
}

\section{Rafael Minatogau Ferro}

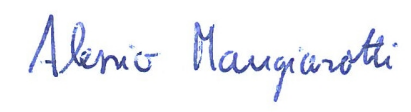

Orientador: Prof. Dr. Alessio Mangiarotti

Tese de doutorado apresentada ao Instituto de Física da Universidade de São Paulo, como requisito parcial para a obtenção do título de Doutor em Ciências.

Banca Examinadora:

Prof. Dr. Alessio Mangiarotti - Orientador (IFUSP)

Profa. Dra. Doris Jakubassa-Amundsen (LMU)

Prof. Dr. Johnny Ferraz Dias (UFRGS)

Prof. Dr. Massimo Chiari (INFN)

Prof. Dr. Vito Roberto Vanin (IFUSP) 
FICHA CATALOGRÁFICA

Preparada pelo Serviço de Biblioteca e Informação do Instituto de Física da Universidade de São Paulo

\section{Ferro, Rafael Minatogau}

Estudo do espectro contínuo de raios- $X$ induzido pela passagem de prótons de alguns MeV na matéria. São Paulo, 2021.

Tese (Doutorado) - Universidade de São Paulo, Instituto de Física, Depto. de Física Experimental.

Orientador: Prof. Dr. Alessio Mangiarotti

Área de Concentração: Física Nuclear

Unitermos: 1. Radiação contínua; 2. Colisões; 3. Íons; 4. Espalhamento. 


\author{
University of São Paulo \\ Physics Institute
}

\title{
Investigation on the continuous X-ray spectrum induced by protons of some $\mathrm{MeV}$ in matter
}

\author{
Rafael Minatogau Ferro
}

Supervisor: Prof. Dr. Alessio Mangiarotti

Thesis submitted to the Physics Institute of the University of São Paulo in partial fulfillment of the requirements for the degree of Doctor of Science.

Examining Committee:

Prof. Dr. Alessio Mangiarotti - Supervisor (IFUSP)

Prof. Dr. Doris Jakubassa-Amundsen (LMU)

Prof. Dr. Johnny Ferraz Dias (UFRGS)

Prof. Dr. Massimo Chiari (INFN)

Prof. Dr. Vito Roberto Vanin (IFUSP) 



\section{Acknowledgment}

We would like to thank Professor Tiago Fiorini da Silva for suggesting the project, his help on the modeling of the detector effects and response function (see Chapter 4), his efforts on measuring the PIXE spectra at LAMFI (see Chapter 5), and his assistance on the interpretations of such data.

We would like to thank Professor José Maria Fernández-Varea for providing the numerical DHFS wave functions and the velocity distributions employed in this work (see Chapter 5). We are also in debt for his help in understanding the theoretical results and for his important contributions to both the published article and the poster presented at the IBA2019 Conference.

The present work has been supported by Conselho Nacional de Desenvolvimento Científico e Tecnológico (CNPq) with the Ph.D. fellowship number 141193/2016-6.

Aos meus pais. 



\begin{abstract}
The bremsstrahlung emission in in proton-atom collisions is studied. For proton energies of a few $\mathrm{MeV}$, five processes are considered: Nuclear Bremsstrahlung (NB), Quasi-Free Electron Bremsstrahlung (QFEB), Atomic Bremsstrahlung (AB), Radiative Ionization (RI), and Secondary Electron Bremsstrahlung (SEB). The concepts involved in these models are reviewed and the expressions currently in use are presented. To evaluate the current models of proton bremsstrahlung and confront the changes proposed in this work, a FORTRAN code has been developed and tested according to the results available in the references. In particular, an alternative expression for the SEB doubly differential cross section (DDCS) is proposed in this work. The ionization DDCS by impact of the proton, which is present in the calculations of the SEB and the $A B+R I D D C S s$, is described in terms of the Binary Encounter Approximation (BEA), being sensitive to the model of the velocity distributions (VD) of the orbital electrons in the target material. In this sense, the calculations of the ionization, SEB, and AB+RI DDCSs are improved in two ways: applying the corresponding hydrogenic VD for the subshells $\mathrm{K}$, L, and M; implementing the numerical VDs from the Dirac-Hartree-Fock-Slater (DHFS) method. Additionally, the PIXE analytical method is presented as the experimental context for the study of proton bremsstrahlung theories, and the relevant aspects of the technique are reviewed. Two PIXE experiments have been performed at LAMFI, USP, for a gold target and proton energies of 2 and $3 \mathrm{MeV}$. In order to compare the theoretical models with the PIXE spectra, the detection effects are analyzed and applied to the calculated spectra. Finally, the theoretical calculations with the DHFS and the hydrogenic VDs are compared to the experimental spectra, and the results obtained with the former model are show to be in better agreement with the data.
\end{abstract}

Keywords:

proton bremsstrahlung; secondary electron bremsstrahlung; proton-impact ionisation; binary encounter approximation; Dirac-Hartree-Fock-Slater; PIXE 



\section{Resumo}

A emissão de bremsstrahlung em colisões próton-átomo é estudada. No caso de prótons com energias da ordem de alguns $\mathrm{MeV}$, cinco processos foram considerados: Nuclear Bremsstrahlung (NB), Quasi-Free Electron Bremsstrahlung (QFEB), Atomic Bremsstrahlung (AB), Radiative Ionization (RI) e Secondary Electron Bremsstrahlung (SEB). Os conceitos necessários para o desenvolvimento desses modelos são revisados e as expressões em uso atualmente são apresentadas. Para calcular os modelos de bremsstrahlung de prótons atuais e validar as mudanças propostas neste trabalho, um código em FORTRAN foi desenvolvido e testado de acordo com os resultados teóricos disponíveis nas referências. Em particular, uma expressão alternativa para a seção de choque duplamente diferencial (DDCS) da SEB é proposta neste trabalho. A DDCS de ionização por impacto do próton, presente nos cálculos das DDCSs da SEB e da AB+RI, é descrita em termos da Binary Encouter Approximation (BEA), sendo sensível ao modelo para a distribuição de velocidades (VD) dos elétrons orbitais no material alvo. Neste sentido, os cálculos das DDCSs de ionização, SEB e AB+RI são melhoradas de duas formas: aplicando a VD hidrogenoide correspondente das subcamadas K, L e M; implementado as VDs numéricas obtidas através do método de Dirac-Hartree-Fock-Slater (DHFS). Ademais, o método analítico PIXE é apresentado como o contexto experimental para o estudo de bremsstrahlung de prótons e os aspectos mais relevantes dessa técnica são revisados. Dois experimentos foram realizados no LAMFI, USP, para um alvo de ouro e energias do feixe de 2 e $3 \mathrm{MeV}$. Para que se possa comparar os modelos teóricos com os espectros PIXE, os efeitos de detecção são analisados e aplicados aos espectros de bremsstrahlung calculados. Por fim, os cálculos teóricos utilizando as VDs de DHFS e hidrogenoide são comparadas aos espectros experimentais e os resultados obtidos com o primeiro modelo apresentam melhor acordo com os dados experimentais.

Palavras-chave:

bremsstrahlung de prótons; secondary electron bremsstrahlung; ionização por impacto de prótons; Dirac-Hartree-Fock-Slater; PIXE 



\section{Sumário}

List of Symbols $\quad$ i

List of Acronyms

1 Introduction 1

2 Bremsstrahlung 9

2.1 General aspects . . . . . . . . . . . . . . . . . 10

2.2 Electron-induced bremsstrahlung . . . . . . . . . . . . . . . 13

2.3 Proton-induced bremsstrahlung . . . . . . . . . . . . . . . . 15

2.3.1 Secondary Electron Bremsstrahlung . . . . . . . . . . . . . . . . . 16

2.3.1.1 Ionization by impact of a proton . . . . . . . . . . . 23

2.3.1.2 Velocity distribution of the electrons . . . . . . . . . 25

2.3.1.3 Energy loss . . . . . . . . . . . . . . . . 29

2.3.2 Atomic Bremsstrahlung and Radiative Ionization . . . . . . . . . . . 32

2.3.3 Quasi-Free Electron Bremsstrahlung . . . . . . . . . . . . . . . 36

2.3.4 Nuclear Bremsstrahlung . . . . . . . . . . . . . . . . . . . . 40

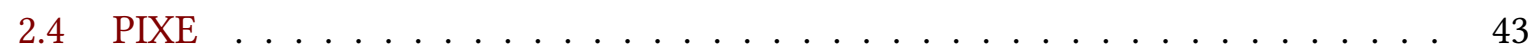

2.4.1 Characteristic x-rays ...................... 44

2.4 PIXE spectroscopy . . . . . . . . . . . . . . . . 46

2.4 .3 Interactions of $\mathrm{x}$-rays with matter $\ldots \ldots \ldots \ldots$

$3 \quad$ Theoretical calculations $\quad 53$

3.1 Secondary Electron Bremsstrahlung . . . . . . . . . . . . . . . 53

3.1 .1 Ionization DDCS . . . . . . . . . . . . . . . . . 54

3.1.2 Comparison between the models for the VD . . . . . . . . . . . 56

3.1.3 Electron bremsstrahlung DDCS . . . . . . . . . . . . . . 57

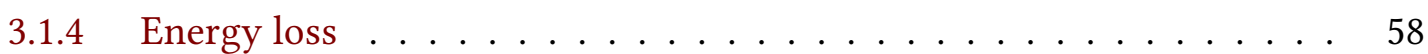

3.1.5 Calculation of the SEB DDCS . . . . . . . . . . . . . 60 
3.2 Code implementation . . . . . . . . . . . . . . . . 63

3.3 Code validation . . . . . . . . . . . . . . . . 67

3.3.1 Ionization DDCS . . . . . . . . . . . . . . . 69

3.3.2 Secondary Electron Bremsstrahlung DDCS . . . . . . . . . . . . . 70

3.3.3 Atomic Bremsstrahlung and Radiative Ionization DDCS . . . . . . . 71

3.3.4 Quasi-Free Electron Bremsstrahlung DDCS . . . . . . . . . . . . 72

3.3.5 Nuclear Bremsstrahlung DDCS . . . . . . . . . . . . . . . . . . 75

4 Experimental measurements $\quad 79$

4.1 Experimental setup . . . . . . . . . . . . . . . 80

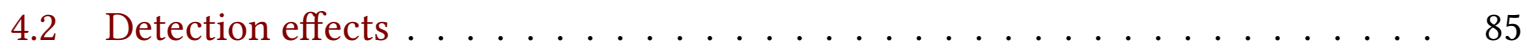

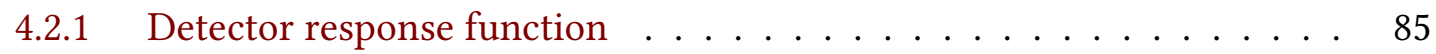

4.2 .2 Detector efficiency . . . . . . . . . . . . . 88

$4.2 .3 \quad$ Pile-up effect . . . . . . . . . . . . . . . . . . 90

4.3 Determination of the parameters of the response function from experiment . . 93

4.4 Estimation of the thickness of the target for future PIXE experiments . . . . . 95

5 Results $\quad 101$

5.1 Calculations with DHFS VDs . . . . . . . . . . . . . . . 102

5.1 .1 Ionization cross sections . . . . . . . . . . . . . . 103

$5.1 .2 \quad$ SEB DDCSs . . . . . . . . . . . . . . . . . 105

$5.1 .3 \mathrm{AB}+\mathrm{RI} \mathrm{DDCSs} \ldots \ldots \ldots \ldots . \ldots \ldots . \ldots \ldots$

5.2 Comparison with experimental data . . . . . . . . . . . . . . . 113

6 Conclusions $\quad 121$

$\begin{array}{ll}\text { Referências Bibliográficas } & 125\end{array}$ 


\title{
List of Symbols
}

\author{
$m_{\mathrm{p}} \quad$ Mass of the proton \\ $E_{\mathrm{p}} \quad$ Kinetic energy of the projectile (proton) \\ $v_{\mathrm{p}}, \beta_{\mathrm{p}} \quad$ Speed of the projectile (proton) \\ $Z_{\mathrm{p}} \quad$ Atomic number of the projectile \\ $m_{\mathrm{e}} \quad$ Mass of the electron \\ $E_{\mathrm{e}}, E_{\mathrm{e}}^{\prime} \quad$ Kinetic energy of the ejected electron \\ $v_{\mathrm{e}}, \beta_{\mathrm{e}} \quad$ Speed of the ejected electron \\ $v, \beta \quad$ Speed of the orbital electron \\ $\Omega_{\mathrm{e}} \quad$ Solid angle of the ejected electron in the reference frame of the laboratory \\ $\theta_{\mathrm{e}} \quad$ Polar angle of the ejected electron in the reference frame of the laboratory \\ $\varphi_{\mathrm{e}} \quad$ Azimuthal angle of the ejected electron in the reference frame of the laboratory \\ $\hbar \omega, E \quad$ Energy of the photon \\ $\Omega \quad$ Solid angle of the photon in the reference frame of the laboratory \\ $\theta \quad$ Polar angle of the photon in the reference frame of the laboratory \\ $\Omega_{\mathrm{br}} \quad$ Solid angle of the photon relative to the direction of the emitting electron \\ $\theta_{\mathrm{br}} \quad$ Polar angle of the photon relative to the direction of the emitting electron \\ $Z \quad$ Atomic number of the target atom \\ $U_{i} \quad$ Binding energy of the $i$-th atomic subshell \\ $N_{i} \quad$ Number of electrons in the $i$-th subshell \\ $\mathcal{N}$ Number of atoms per unit volume of the target material \\ $\bar{I} \quad$ Mean excitation energy \\ $a_{0} \quad$ Bohr radius
}


$r_{0} \quad$ Classical electron radius

$\alpha \quad$ Fine-structure constant 


\title{
List of Acronyms
}

\author{
$\mathrm{AB} \quad$ Atomic bremsstrahlung \\ NB Nuclear bremsstrahlung \\ QFEB Quasi-free electron bremsstrahlung \\ RI Radiative ionization \\ SEB Secondary electron bremsstrahlung \\ BEA Binary encounter approximation \\ PWBA Plane wave Born approximation \\ ERD Elastic recoil detection \\ IBA Ion beam analysis \\ PIXE Particle induced x-ray emission \\ RBS Rutherford backscattering \\ DDCS Doubly differential cross section \\ DHFS Dirac-Hartree-Fock-Slater \\ PDF Probability density function \\ VD Velocity distribution \\ LAMFI Laboratório de Análise de Materiais e Feixes Iônicos
}





\section{Capítulo 1}

\section{Introduction}

The process of emission of radiation by a scattered particle is referred to by the German term bremsstrahlung, which means braking radiation. The name was proposed by Sommerfeld, in reference to the first observations of the phenomenon when high-energy electrons were braked by a thick metallic target. The first interpretation, suggested by Sommerfeld in 1909, was that of a linear deceleration of the charged particle producing radiation according to Maxwell equations.

One interesting classical interpretation of this phenomenon is that described by Purcell and Morin (2013), based on the postulate of the limit imposed by the speed of light, $c$, on the propagation of electric and magnetic field configurations.

Consider, for example, the case of an electron which has been moving with a constant velocity $v_{0} \ll c$ along an axis $x$. When the electron reaches $x=0$, at time $t=0$, it is decelerated. If the deceleration is abrupt and the electron remains at rest, its electric field lines suddenly transform into those of an electron at rest. However, after a time $T$, a point in space at a distance of the electron further than $R=c T$ still has the field lines of a moving particle, pointing at the location where the electron would be had it not been stopped. In this case, the field lines inside and outside the sphere of radius $R$ can be connected by considering the Gauss law.

Figure 1.1 (Purcell and Morin, 2013) illustrates the situation described above. The dashed path defined by $E A B C D F$, when rotated around the $x$ axis, generates a surfaces through which the total flux must be zero. In this way, it is possible to relate the angles $\theta_{0}$ and $\phi_{0}$ by the expression $\tan \theta_{0}=\gamma \tan \phi_{0}$ (Purcell and Morin, 2013), where $\gamma=\left(1-v_{0}^{2} / c^{2}\right)^{-1 / 2}$. The surface of the sphere defined by the transition of the field lines from $\theta_{0}$ to $\phi_{0}$ expands with the speed of light. With that, a transverse magnetic field emerges, forming the electromagnetic radiation emitted by the electron.

On the other hand, if the electron takes a time $\tau \ll T$ to be completely stopped, it experiences a constant deceleration of magnitude $a=v_{0} / \tau$. In this case, there are three regions 


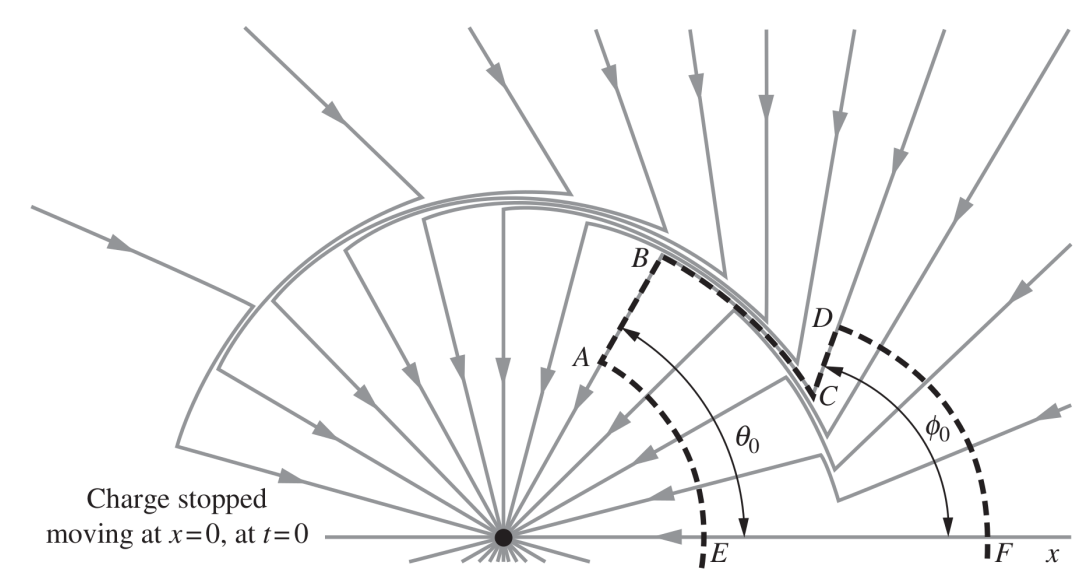

Figura 1.1: Electric field lines of an electron that has been moving with constant velocity $v_{0} \ll c$ up to $x=0$, where it is abruptly stopped. Source: Purcell and Morin (2013)

in space, depicted in Figure 1.2 (Purcell and Morin, 2013), in which the field lines have different configurations:

- Region I, beyond the sphere with radius $R=c T$, in which the field lines correspond to those of the electron in motion with position $x=v_{0} T$, i.e. where it would be if it had not been decelerated;

- Region II, inside the sphere with radius $R=c(T-\tau)$, where the field lines are those of the actual position of the electron at rest in $x=\frac{1}{2} v_{0} \tau$; and

- Region III, the Shell between Regions I and II, in which the field lines connect the outer and inner lines.

The field lines are thus indicated by the path $A B C D$ in Figure 1.2, and the line $B C$ corresponds to the field $E$ inside the shell. The field $E$ is composed of a radial component $E_{r}$ and a transverse component $E_{\theta}$, the ratio between them being

$$
\frac{E_{\theta}}{E_{r}}=\frac{v_{0} T \sin \theta}{c \tau}
$$

But the radial component is simply given by $E_{r}=q / 4 \pi \epsilon_{0} R^{2}$, in SI, where $q$ is the charge of the electron and $\epsilon_{0}$ is the dielectric constant of vacuum. Therefore,

$$
E_{\theta}=\frac{v_{0} T \sin \theta}{c \tau} E_{r}=\frac{q a \sin \theta}{4 \pi \epsilon_{0} c^{2} R}
$$

With Equation (1.0.2), it is possible to calculate the energy density in the shell, which is proportional to $|E|^{2}$. Then, the energy density can be integrated over the solid angle in order to obtain the total radiated power. Finally, by multiplying the result by a factor of 2 , which 


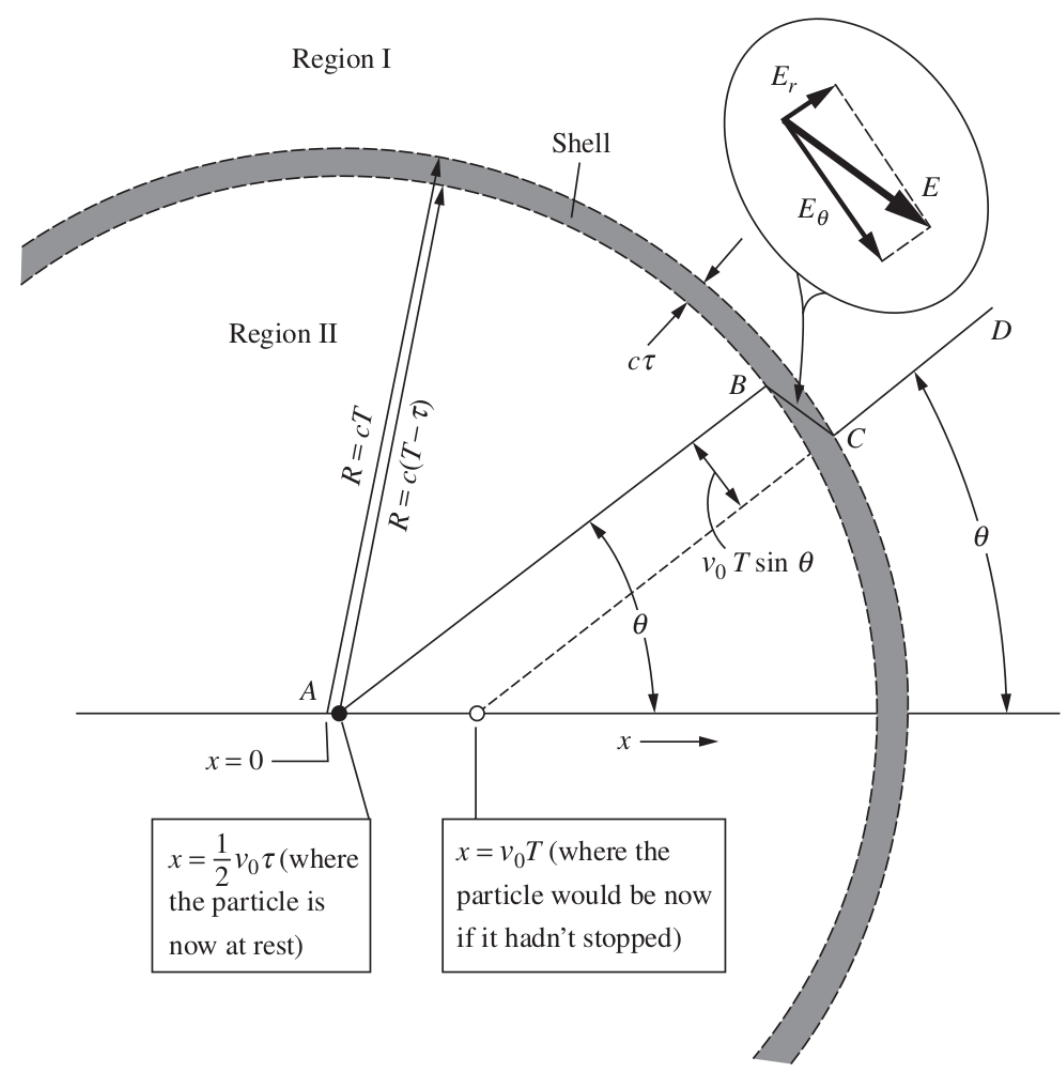

Figura 1.2: Electric field lines of an electron that has been decelerated within a time $\tau \ll T$. Source: Purcell and Morin (2013)

takes into account the same amount of energy from the corresponding magnetic field, the Larmor formula is obtained. The Larmor formula is valid for nonrelativistic particles and is reviewed in Section 2.1.

With the advent of the old quantum mechanics, based on the Plank postulate and the correspondence principle, Sommerfeld tried to relate the breaking distance, the main unknown of the theory, to Plank constant. To produce a bremsstrahlung radiation with an intensity compatible with experiment, the breaking distance had to be much smaller than the atomic dimensions. When the Bohr atomic model was introduced, this became untenable, and, around 1926, Sommerfeld developed a theory closely mimicking that of the Bohr atom, where the bremsstrahlung radiation is emitted during transition between hyperbolic (open) motions. With the establishment of the new quantum mechanics, and in particular the Schrödinger equation, Sommerfeld could finally put the theory of bremsstrahlung emission on firm grounds and developed the first exact calculation of the cross section starting from the exact solution of the Schrödinger equation for a pure Coulomb field (Sommerfeld, 1931). The extension of bremsstrahlung calculations to the Dirac equation to account for relativistic electrons and then to modern quantum electrodynamics to include radiative corrections is too long to be reviewed 
here, but can be found elsewhere (Haug and Nakel, 2004; Mangiarotti and Martins, 2017). Sommerfeld actively participated in these efforts as well, introducing the famous SommerfeldMaue wave functions, that are an approximate solution of the Dirac equation for a pure Coulomb field.

The problem of the lack of bremsstrahlung by particles heavier than the electron was actually already tackled in the Ph.D. dissertation by one of Sommerfeld students, Scherzer (1932), who employed the semi-classical theory introduced by Sommerfeld in 1926. It became clear that, since the forces have similar magnitudes in electron-nucleus and proton-nucleus bremsstrahlung, but the accelerations are smaller, the emission intensity in the former case ends up being approximately suppressed by the ratio of the electron to proton mass squared.

However the situation is not so simple from the experimental point of view: targets are typically made of neutral atoms. Thus, when light ions, like protons, impinge on them, the projectile interacts not only with the Coulomb filed of the nucleus but also with the atomic electrons and induces several bremsstrahlung processes. In particular, the impact ejects electrons from the atoms: these particles can also emit radiation. Because of the mentioned suppression factor, this radiation by secondary electrons is actually much more intense than the direct proton bremsstrahlung in the Coulomb field of the nucleus. The experimental difficulty of having a clear access to proton bremsstrahlung alone and the theoretical complexity of having to deal with the sum of several processes explains the sporadic interest that proton bremsstrahlung has received over the years. We hope the present work contributes to revert such a tendency: as it will become clear, there are ample margins for progress on the theoretical description and a great lack of accurate data to benchmark those advances.

The subject is also of great significance for many areas of physics, besides having multiple practical applications. As an example, it can be mentioned that some methods of material analysis impinge charged particles, such as electrons and ions with appropriate energies, towards a solid target, thereby producing a radiation that gives some information about the specimen. Such an approach gives origin to an important family of modern analytical techniques called Ion Beam Analysis (IBA) (Jeynes et al., 2011). Among several options available in IBA, one can cite the Rutherford Backscattering (RBS), which is sensitive to heavy elements in the specimen; the Elastic Recoil Detection (ERD), which is applicable to light elements; and the Particle Induced X-ray Emission (PIXE), which uses light ions to analyze the target material. Bremsstrahlung emission represents a background process in PIXE and, in many cases, sets the ultimate limit to the minimum element concentration that can be detected.

In this context, the object of study of the present work are the bremsstrahlung processes induced by the protons of a few $\mathrm{MeV}(1-4 \mathrm{MeV})$, when impinging on thin targets. This energy has been selected because it allows to compare with data collected at the Laboratório de Análise 
de Materiais e Feixes Iônicos (LAMFI) of the Institute of Physics of the University of São Paulo (IF USP). Therefore, five radiative processes are considered in this work (Ishii, 2006): Nuclear Bremsstrahlung (NB), Quasi-Free Electron Bremsstrahlung (QFEB), Atomic Bremsstrahlung (AB), Radiative Ionization (RI), and Secondary Electron Bremsstrahlung (SEB).

Among these processes of bremsstrahlung production, SEB and $\mathrm{AB}$ give the greatest contributions. SEB was first investigated by Folkmann et al. (1974), Jakubaßa-Amundsen and Kleber (1975) and Ishii et al. (1976). Later work by Yamadera et al. (1981) presents a more explicit expression for the SEB DDCS, based on the same formalism by Ishii et al. (1976), which reduces the dimension of the final integral to be performed numerically needed in the previous works.

In the present work, an expression for the SEB DDCS in terms of the exponential integral function is proposed. With this approach, the dimension of the numerical integration is reduced from a $5 \mathrm{D}$ integral to a $3 \mathrm{D}$ integral and the results are shown to agree with those by Ishii et al. (1976).

The SEB DDCSs implicitly rely on the ionization process, which, in the approach by Ishii et al. (1976), has been calculated within the binary encounter approximation (BEA) (Bonsen and Vriens, 1970; Folkmann et al., 1975). These DDCSs for the ejected electron angle and energy are interesting by themselves and are investigated as well in the present work.

In the BEA, the target electron is assumed to be free and the nucleus establishes its initial momentum distribution, i.e. its velocity distribution (VD). It is thus expected that the ionization DDCS in the BEA might be sensitive to the choice of the VD of the target electrons. As a consequence, the SEB DDCS must change according to the VD implemented in the calculations of the ionization DDCS. Similarly, the AB and RI DDCSs are connected to the ionization process and are thus affected by the VD model.

Current calculations of the SEB and the AB+RI DDCSs assume the VD of the orbital electrons to be given approximately by the nonrelativistic hydrogenic 1s wave function (Folkmann et al., 1974; Ishii et al., 1976; Yamadera et al., 1981). This approximation, however, may not be accurate for elements with higher atomic number in the cases of outer shells, where the screening of the Coulomb field of the nucleus by inner ones is important and of inner shells as well, where relativistic effects are not negligible. In the present work, the VD of the orbital electrons from the wave functions obtained numerically by the Dirac-Hatree-Fock-Slater (DHFS) method are employed. They have been provided by J. M. Fernández-Varea, from Universitat de Barcelona ${ }^{1}$. Moreover, for the K, L, and M shells, the 1s hydrogenic VD has been replaced with the corresponding hydrogenic VD of each subshell. The calculations of the ionization, the

\footnotetext{
${ }^{1}$ Facultat de Física (FQA and ICC), Universitat de Barcelona. Diagonal 645, ES-08028 Barcelona, Catalonia, Spain
} 
SEB, and the AB+RI DDCSs based on DHFS VDs are compared with the results relying on the hydrogenic VDs.

In order to implement the calculations of the proton bremsstrahlung DDCSs, a FORTRAN code has been developed. The program is intended to perform different types of calculations within the validity of the implemented theories and has been validated through direct comparisons with the data available in the reference papers.

Additionally, the calculations are compared with data obtained at LAMFI. The experiment consists of the PIXE technique, which focuses a beam of protons on a target and relies on spectroscopy techniques to analyze the characteristic x-rays produced thereby. The measurements have been conducted by T. F. da Silva, at LAMFI. However, in order to compare the calculated bremsstrahlung cross sections with the experimental spectra, a convolution with the response function and the efficiency of the detector used must be performed. It is thus necessary to consider the intrinsic detection effects, as well as some electronic aspects of the equipment available at LAMFI.

A lithium-drift silicon detector, $\mathrm{Si}(\mathrm{Li})$, is employed in the LAMFI setup. Therefore, a model for its response function is presented, along with the methods used to calibrate the necessary parameters. The attenuation in the target, the detector efficiency, and the effect of pile-up in the electronic chain also need to be described.

Finally, these effects are applied to the theoretical calculations of the bremsstrahlung and characteristic x-rays cross sections (which involves the ionization cross sections, fluorescence yields, Coster-Kronig transition rates, and line emission rates), thus enabling a qualitative comparison between the theories and the data. The main purpose is to analyze the impact of the DHFS VDs on the bremsstrahlung spectrum in comparison with those obtained using the hydrogenic VDs.

The content of this thesis is organized as follows.

In Chapter 2, the general aspects of the bremsstrahlung process are reviewed. The five proton bremsstrahlung mechanisms are discussed therein, along with the concepts related to the available theories and the expressions currently in use. Particularly when addressing the SEB process, the concepts of ionization by impact of a proton, velocity distribution of the orbital electrons, and energy loss are discussed.

The PIXE method is also presented in Chapter 2, as it provides the experimental data to be compared with the proposed theoretical calculations. In this context, the mechanism of characteristic x-ray emission, the photoelectric absorption, and the Compton scattering effects are briefly studied. These subjects are important for later discussing the detection effects involved in the PIXE spectroscopy and thus analyzing the experimental spectra.

Chapter 3 presents the main object of this thesis: the calculation of the SEB DDCS in 
terms of an exponential integral function and the calculation of the ionization DDCS in the BEA based on the DHFS VDs. These implementations are compared with the currently accepted expressions for the SEB DDCS adopting the hydrogenic VDs. Besides, the main aspects of the numerical calculations implemented in this work are presented, along with the steps performed to validate them with published literature. Moreover, the DHFS and the hydrogenic VDs are compared and analyzed, and the numerical implementation of the total ionization cross sections calculated with the hydrogenic VDs are verified against equivalent results obtained separately by J. M. Fernández-Varea.

The experimental setup employed for the measurements performed at LAMFI is presented in Chapter 4. The detection effects inherent to the PIXE spectroscopy and needed for analyzing the current results are introduced. Then, the method utilized for the determination of the parameters involved in the modeling of the detector effects is presented. Basically, the $\mathrm{x}$-ray peaks are calculated and the results are compared to the data adjusting the parameters mentioned.

Finally, in Chapter 5, the results of the implementation of the DHFS VDs are compared to the calculations with the hydrogenic VDs. The analysis is presented separately for the ionization, the SEB, and the AB+RI DDCSs, showing the individual shells and the final sum over all of them. Furthermore, the confrontation between the experimental spectra and the calculated DDCSs is discussed in details. Some of the results presented in this chapter, namely those concerning the influence of the VDs on the ionization and the SEB DDCSs, along with the proposed expression for the SEB DDCSs, have been presented at the IBA 2019 conference and are already published (Ferro et al., 2020).

At last, a brief conclusion is presented in Chapter 6 and further perspectives for the subject of this work are considered. 



\section{Capítulo 2}

\section{Bremsstrahlung}

Bremsstrahlung is the process of radiation emission by a charged particle, typically when it is scattered; but other contributions are possible, as it will be discussed below. This phenomenon has many applications in experimental research, including multiple techniques of analysis of materials. One technique is the PIXE method, where a beam of protons (light ions, in general) is focused on the target material to be analyzed. As a consequence, the impinging proton induces the production of bremsstrahlung, creating a continuous spectrum, which is an important factor contributing to the detection limit in the PIXE spectroscopy.

Nonetheless, in proton-atom collisions, besides the production of bremsstrahlung in the scattering of the proton itself, electrons are ejected from the target atom by the impact of the projectile. Consequently, the ejected electrons, called secondary electrons, also produce bremsstrahlung. In fact, five mechanisms of proton-induced bremsstrahlung are considered for proton energies of 0.5-40 MeV: Nuclear Bremsstrahlung, Secondary Electron Bremsstrahlung, Atomic Bremsstrahlung, Radiative Ionization, and Quasi-Free Electron Bremsstrahlung. These processes of bremsstrahlung production are studied in more detail in this Chapter.

Moreover, in the PIXE technique, the impact of the proton ejects an electron from the inner shell of a target atom. The vacancy thus created can be filled by an electron from an outer shell, possibly generating a cascade of rearrangements, in which case the process can be followed by the emission of one or more characteristic x-ray photons or one or more atomic electrons (called Auger electrons in such a context) to eliminate the excess excitation energy. In this way, the analysis of the PIXE spectrum can give information about the composition of the sample. Some theoretical aspects involved in characteristic x-ray production must be reviewed as well, since they have to be taken into account for a meaningful comparison of proton bremsstrahlung calculations to experiment.

Therefore, in this Chapter, the processes of proton bremsstrahlung are reviewed and the PIXE analytical method is discussed as the experimental context to the present work. 
In Section 2.1, a brief review of the aspects of radiation emission by accelerated charges is presented. The electron bremsstrahlung is reviewed in Section 2.2 and the processes of protoninduced bremsstrahlung are studied in Section 2.3, besides the concepts necessary to their calculations. Finally, Section 2.4 discuss the PIXE analytical method as the main technique where the proton bremsstrahlung processes might be of great importance.

\subsection{General aspects}

In classical electrodynamics, it can be shown that charged particles emit radiation when they are submitted to accelerations. A usual situation for radiation emission by accelerated charges is that of scattering. In the collision of two charged particles, the main force acting on them is due to the Coulomb field, and they both emit radiation.

The simplest understanding of the emission of radiation by moving charges can be grasped considering the case of a point charge in a linear accelerated motion. In classical theory, the total radiated power is proportional to the squared charge and the squared acceleration, as can be seen by the Larmor formula for nonrelativistic particles

$$
P=\frac{2}{3} \frac{q^{2} a^{2}}{c^{3}}
$$

where $q$ is the charge and $a$ is the acceleration, in cgs units. The angular distribution of radiation emitted by an accelerated charge is given by

$$
\frac{\mathrm{d} P}{\mathrm{~d} \Omega}=\frac{q^{2} a^{2}}{4 \pi c^{3}} \sin ^{2} \theta
$$

where $\theta$ is the emission angle relative to the direction of acceleration. As expected, Equation (2.1.1) is obtained by integrating Equation (2.1.2) over the solid angle. It can be seen that the maximum radiation emission occurs at right angles to the acceleration, but no radiation is emitted along that axis.

This approach is only acceptable for low velocities of the incident particles $\left(\beta_{\mathrm{p}} \approx 0\right)$. For higher energies, the radiation fields also depend on the velocity of the particle, and so does the angular distribution. Again, the simplest instance is to consider a linear motion, where the velocity and the acceleration vectors are parallel. In this case, the relativistic angular distribution is given by (Jackson, 1999)

$$
\frac{\mathrm{d} P\left(t^{\prime}\right)}{\mathrm{d} \Omega}=\frac{q^{2} a^{2}}{4 \pi c^{3}} \frac{\sin ^{2} \theta}{\left(1-\beta_{\mathrm{p}} \cos \theta\right)^{5}},
$$




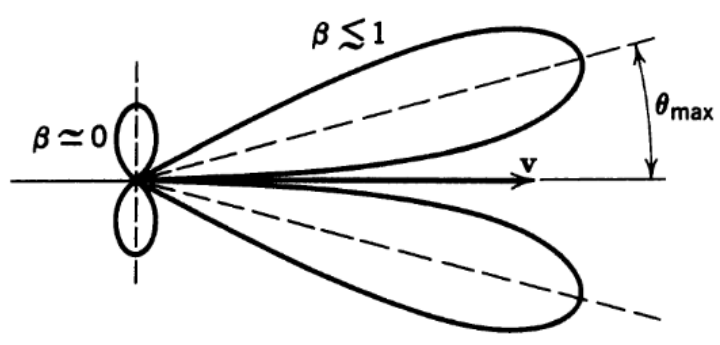

Figura 2.1: Radiation patterns for $\beta \approx 0$ and $\beta \leq 1$, with parallel velocity and acceleration directions. Not to scale. Source: Jackson (1999).

which is the relativistic generalization of Equation (2.1.2) and thus reduces to the nonrelativistic result when $\beta_{\mathrm{p}} \ll 1$.

Equation (2.1.3) shows that the angular distribution is no longer symmetric about $\theta=90^{\circ}$. It actually becomes forward peaked and its magnitude increases, as represented in Figure 2.1 (Jackson, 1999). The maximum intensity occurs at

$$
\theta_{\max }=\cos ^{-1}\left[\frac{1}{3 \beta_{\mathrm{p}}}\left(\sqrt{1+15 \beta_{\mathrm{p}}^{2}}-1\right)\right]
$$

As $\beta_{\mathrm{p}} \rightarrow 1, \theta_{\max }$ is very small, and the angular distribution is peaked in the direction of motion. The first interpretation of bremsstrahlung emission was given by Sommerfeld in 1909 considering a linearly decelerated charge. However, it was soon realized that, to explain the characteristic of the emission, the electrons would have to be stopped over distances of the order of a small fraction of the atomic or molecular dimensions, as mentioned in Chapter 1.

A large acceleration is actually present during the deflection of a charged particle by the Coulomb field of the nucleus. This realization by Sommerfeld around 1926 was a great breakthrough in bremsstrahlung theory. In the scattering of a light particle, charge $Z_{\mathrm{p}} e$, mass $m_{\mathrm{p}}$, and initial velocity $c \beta_{\mathrm{p}}$, by a heavy nucleus, charge $Z e$, the recoil taken by the latter is negligible, due to its large mass. Since the acceleration of the incident particle is much larger than that of the recoiling nucleus, the radiation emitted from the former is more intense and the collision can be treated as the scattering of a charged particle by a fixed Coulomb field. In this case the elastic scattering can be expressed as a cross section per momentum transfer, $Q$, given by the Rutherford formula (Jackson, 1999)

$$
\frac{\mathrm{d} \sigma_{\mathrm{s}}}{\mathrm{d} Q}=8 \pi\left(\frac{Z Z_{\mathrm{p}} e^{2}}{c \beta_{\mathrm{p}}}\right)^{2} \frac{1}{Q^{3}}
$$

where $Q^{2}=\left|\boldsymbol{p}^{\prime}-\boldsymbol{p}\right|^{2}=4 p^{2} \sin ^{2}\left(\theta^{\prime} / 2\right), E=p^{2} / 2 m_{\mathrm{p}}$ and $E^{\prime}=p^{\prime 2} / 2 m_{\mathrm{p}}$ are the kinetic energies of the incident particle before and after the collision, and $\theta^{\prime}$ is the scattering angle of the particle. 
The differential radiation cross section can be described as

$$
\frac{\mathrm{d}^{2} \chi}{\mathrm{d} \omega \mathrm{d} Q}=\frac{\mathrm{d} I}{\mathrm{~d} \omega} \frac{\mathrm{d} \sigma_{\mathrm{s}}}{\mathrm{d} Q}
$$

where $d I / d \omega$ is the radiated energy per unit frequency given by (Jackson, 1999)

$$
\frac{\mathrm{d} I}{\mathrm{~d} \omega}=\frac{2}{3 \pi} \frac{Z_{\mathrm{p}}^{2} e^{2}}{m_{\mathrm{p}}^{2} c^{3}}
$$

which is valid for small frequencies and momentum transfers. Combining the above equations, (2.1.5)-(2.1.7), and integrating over the momentum transfer gives the radiation cross section

$$
\begin{aligned}
\frac{\mathrm{d} \chi}{\mathrm{d} \omega} & =\frac{16}{3} \frac{Z^{2} e^{2}}{c}\left(\frac{Z_{\mathrm{p}}^{2} e^{2}}{m_{\mathrm{p}} c^{2}}\right)^{2} \frac{1}{\beta_{\mathrm{p}}^{2}} \int_{Q_{\min }}^{Q_{\max }} \frac{\mathrm{d} Q}{Q} \\
& =\frac{16}{3} \frac{Z^{2} e^{2}}{c}\left(\frac{Z_{\mathrm{p}}^{2} e^{2}}{m_{\mathrm{p}} c^{2}}\right)^{2} \frac{1}{\beta_{\mathrm{p}}^{2}} \ln \left(\frac{Q_{\max }}{Q_{\min }}\right)
\end{aligned}
$$

In Equation (2.1.8), the maximum and minimum momentum transfer are determined by the kinematics, and are given by $Q_{\max }=p+p^{\prime}$ and $Q_{\min }=p-p^{\prime}$. Additionally, because of the conservation of energy, $E=E^{\prime}+\hbar \omega$, where $\hbar \omega$ is the energy of the emitted photon. With these expressions, the ratio $Q_{\max } / Q_{\min }$ can be written as

$$
\frac{Q_{\max }}{Q_{\min }}=\frac{p+p^{\prime}}{p-p^{\prime}}=\frac{(\sqrt{E}+\sqrt{E-\hbar \omega})^{2}}{\hbar \omega} .
$$

Finally, the above result can be used in Equation (2.1.8) to obtain

$$
\frac{\mathrm{d} \chi}{\mathrm{d} \omega}=\frac{16}{3} \frac{Z^{2} e^{2}}{c}\left(\frac{Z_{\mathrm{p}}^{2} e^{2}}{m_{\mathrm{p}}^{2} c^{2}}\right)^{2} \frac{1}{\beta_{\mathrm{p}}^{2}} \ln \left[\frac{(\sqrt{E}+\sqrt{E-\hbar \omega})^{2}}{\hbar \omega}\right]
$$

In classical electrodynamics, radiation is always emitted when a charged particle is accelerated, but the energy and momentum of the irradiated electromagnetic wave are, of course, not subject to the quantization conditions expected for the emission of a photon. Quantum mechanics is needed to correctly describe this effect and then the bremsstrahlung process is treated as a certain probability of photon emission by the scattered particle. Nevertheless, the previous result agrees with the calculations from the Born approximation obtained by Bethe and Heitler (1934) for the scattering of an electron, as discussed in the next Section 2.2. 


\subsection{Electron-induced bremsstrahlung}

As mentioned above, the scattering of an electron in the Coulomb field of a target atom leads to bremsstrahlung production, denoted as the electron-nucleus bremsstrahlung. The incident electron may also directly interact with atomic electrons and, in this case, the radiative process is called electron-electron bremsstrahlung. The latter is proportional to $Z$, while the electron-nucleus contribution is proportional to $Z^{2}$. For a first attempt, electron-electron bremsstrahlung is neglected, because the treatment is much more complicated than that of the electron-nucleus process. Although this approximation is possibly not accurate for low- $\mathrm{Z}$ samples, the electron-electron bremsstrahlung cross section is considerably smaller than that of electron-nucleus bremsstrahlung for relatively high atomic numbers of the target.

Therefore, in this work, only the electron-nucleus radiative collisions are considered, and it will be henceforward referred to as electron bremsstrahlung only, so it can be easily distinguished in the context of proton-induced bremsstrahlung processes. Additionally, in the present section only, the subscript $p$ denoting the variables related to the projectile is dropped, simplifying the notations, in contrast with the definition in the List of Symbols, which is used in all other parts of the text.

The electron bremsstrahlung cross section was derived by Bethe and Heitler (1934) on the basis of the first Born approximation. In this approach, the interaction between the electron and the Coulomb field of the target atom is treated as a perturbation, so the distortion to the initial and final electron wave functions is considered to be weak (Haug and Nakel, 2004; Mangiarotti and Martins, 2017). In the case of a pure Coulomb field, without the screening due to the orbital electrons of the target atom, the potential is $V=-Z e^{2} / r$. Then, the wave function $\psi(\boldsymbol{r})$ is expanded as a series in powers of $Z e^{2}$

$$
\psi=\phi_{0}+\left(Z e^{2}\right) \phi_{1}+\left(Z e^{2}\right)^{2} \phi_{2}+\cdots
$$

where $\phi_{0}$ is the initial plane wave and the other terms represent the distortion caused by the interaction with the field.

In this approximation, the transition matrix elements can be calculated and the electron bremsstrahlung cross section, in the nonrelativistic limit, is given by (Heitler, 1954)

$$
\frac{\mathrm{d} \sigma}{\mathrm{d}(\hbar \omega)}=\frac{16}{3} \frac{e^{2}}{\hbar c}\left(\frac{Z e^{2}}{m_{\mathrm{e}} c^{2}}\right)^{2} \frac{1}{\beta^{2}} \frac{1}{\hbar \omega} \ln \left[\frac{(\sqrt{E}+\sqrt{E-\hbar \omega})^{2}}{\hbar \omega}\right]
$$

This expression is related to Equation (2.1.10) by a factor $\hbar^{2} \omega$, so that it is expressed in terms 
of the energy of the photon, whereas Equation (2.1.10) is defined for the frequency.

A necessary condition for the Born series to converge and hence for Equation (2.2.2) to be valid is given by

$$
\frac{\alpha Z}{\beta_{0}} \ll 1 \quad, \quad \frac{\alpha Z}{\beta} \ll 1,
$$

with $\beta_{0}$ and $\beta$ the initial and final electron velocities. However, as discussed in Section 2.1, an equivalent result has been demonstrated by Jackson (1999) using semi-classical arguments and the aspects of the kinematics (see Equation (2.1.10)). Thus, Equation (2.2.2) has an applicability that goes somewhat beyond the convergence condition of the Born series.

Equation (2.2.2) shows that the bremsstrahlung cross section decreases roughly as $1 / \hbar \omega$ and vanishes at the high-energy limit $\hbar \omega=E$. Equation (2.2.2) is valid in the first Born approximation for the pure Coulomb field of a target nucleus of charge $Z e$, without considering the screening, due to the orbital electrons of the target atom, and the Coulomb correction, accounting for the strong distortion of wave functions close to the atomic nucleus.

For small initial or final electron energies, the assumption of plane waves for the electron wave function is no longer valid. In this case, it is necessary to use the wave functions for the continuous spectrum of the Schrödinger equation, as first demonstrated by Sommerfeld (1931). An approximate approach is to multiply Equation (2.2.2) by the correction term (Heitler, 1954; Sommerfeld, 1931)

$$
g\left(\xi_{0}, \xi\right)=\frac{\xi\left(1-e^{-2 \pi \xi_{0}}\right)}{\xi_{0}\left(1-e^{-2 \pi \xi}\right)},
$$

where $\xi_{0}=\alpha Z / \beta_{0}$ and $\xi=\alpha Z / \beta$. With this factor, for small $k$ (i.e. $\left.\beta_{0} \approx \beta\right), g\left(\xi_{0}, \xi\right) \approx 1$, except near the high-energy limit, when $\beta \rightarrow 0, g\left(\xi_{0}, \xi\right) \rightarrow \infty$ and the cross section tends to a finite value.

Bethe and Heitler (1934) also derived the electron bremsstrahlung cross section for the relativistic case and including the screening effect. The resulting expression is more general and it is exact within the validity of the first Born approximation. Other approaches to solving the transition matrix element are available, including a numerical procedure, based on the S-matrix formalism, which uses an expansion in partial waves (Haug and Nakel, 2004; Mangiarotti and Jakubaßa-Amundsen, 2017; Mangiarotti and Martins, 2017). This approach led to the most accurate values of electron bremsstrahlung cross section, especially those calculated by Pratt et al. (1977). In this method, however, as the number of partial waves grows with the electron energy, the total number of terms required to obtain reliable results increases exponentially. Consequently, the calculations performed by Pratt et al. (1977) have been limited to energies of 
the electron up to $2 \mathrm{MeV}$.

As will be seen in Section 2.3.1, despite its limitations, an approximation of Equation (2.2.2) has been used in all works published until the present date for the calculations of secondary electron bremsstrahlung induced by the impact of protons.

\subsection{Proton-induced bremsstrahlung}

When heavy charged particles are accelerated against a solid target, production of bremsstrahlung and characteristic x-rays or Auger electrons from target atoms is induced. While the incident particles can be protons, $\alpha$ particles, or heavy ions, the current work intends to study the continuous $\mathrm{x}$-rays produced in proton-atom collisions. Hence, in the following text, terms like "projectile" or "incident beam" refer generically to the case of protons.

The impact of the incident particles eject electrons from the innermost shells of the target atoms. Then, electrons from outer shells fill those vacancies, thus resulting in an excess excitation energy. Such an energy must be released for the atom to return to its ground state. This is achieved by emitting either x-ray photons or atomic electrons, termed Auger electrons, in accordance with the selection rules of quantum mechanics. Experimentally, it is typically easier to observe the ensuing $\mathrm{x}$-rays. Therefore, the characteristic $\mathrm{x}$-ray spectrum is determined by the energy levels of the elements in the target.

In addition to characteristic x-rays or Auger electrons, projectile scattering in the Coulomb field of target atoms results in bremsstrahlung production. Moreover, electrons participate in the emission of radiation. The resulting spectrum is thus composed of characteristic peaks superimposed on a continuous background.

In this sense, five radiative processes are considered to explain the bremsstrahlung production in proton-atom collisions, for projectile energies between 0.5 and $40 \mathrm{MeV}$ (Ishii, 2006). These processes account for the proton-nucleus bremsstrahlung itself, as well as electron bremsstrahlung mechanisms. Briefly, the five radiative processes are:

Nuclear Bremsstrahlung (NB) : is the radiation produced by the projectile through Coulomb scattering in the field of the target atoms;

Quasi-Free Electron Bremsstrahlung (QFEB) : is the radiation produced by target electrons in the Coulomb field of the incident proton;

Atomic Bremsstrahlung (AB) : is the radiation produced by an electron bound to an atomic field when excited to highly bound states or to the continuum, sequentially returning to its original state; 
Radiative Ionization (RI) : as in the previous process, $A B$, the electron bound to an atomic field is excited to the continuum and produces bremsstrahlung radiation, but does not return to its bound state;

Secondary Electron Bremsstrahlung (SEB) : when the electron is ejected to the continuum, it loses energy as it passes through the target material, subsequently producing radiation when scattered by the Coulomb field of another target atom.

When considering bremsstrahlung production in heavy-ion-atom collisions, other processes might be taken into account, such as Molecular Orbital x-rays (MO) (Anholt, 1985) and Radiative Electron Capture (REC) (Schnopper et al., 1974). They are important bremsstrahlung mechanisms for heavy ions due to their strong dependence on the projectile atomic number, but are negligible in light-ion-atom collisions. Therefore, such processes are not included in this work.

The proton-induced bremsstrahlung processes are calculated as doubly differential cross sections (DDCS), in terms of the energy and the solid angle of emission of the photon, for the collision with a single atom. In order to study the calculation of these DDCSs, it is important to review the concepts and the state-of-the-art of the ionization by impact of a proton, the probability density function (PDF) of the speed of the orbital electrons, or the velocity distribution (VD) of the electrons, and, particularly for the SEB process, the energy loss of a particle (electron) as it passes through the material. Although these concepts will be discussed in the context of the SEB mechanism, the first two are also important for the calculation of the $\mathrm{AB}+\mathrm{RI}$ and QFEB DDCS.

As will be studied throughout the text, the VD of the electrons is an important aspect of the calculation of the ionization DDCS, which has direct effects on the SEB DDCS. In Subsection 2.3.1.2, the theoretical aspects of the calculation of the VD of electron from the nonrelativistic and relativistic wave functions are reviewed. In Chapter 3, a comparison between the models for the VD is presented.

Finally, the five aforementioned bremsstrahlung processes in proton-atom collision will be discussed in detail in the following Sections.

\subsubsection{Secondary Electron Bremsstrahlung}

The Secondary Electron Bremsstrahlung (SEB) was first studied by Folkmann et al. (1974), Jakubaßa-Amundsen and Kleber (1975), and Ishii et al. (1976), and it is described in two steps: first, the projectile ejects an electron from a target atom, which loses energy as it passes through the material; then, the secondary electron produces bremsstrahlung when scattered by the Coulomb field of another target nucleus, as illustrated in Figure 2.2. 


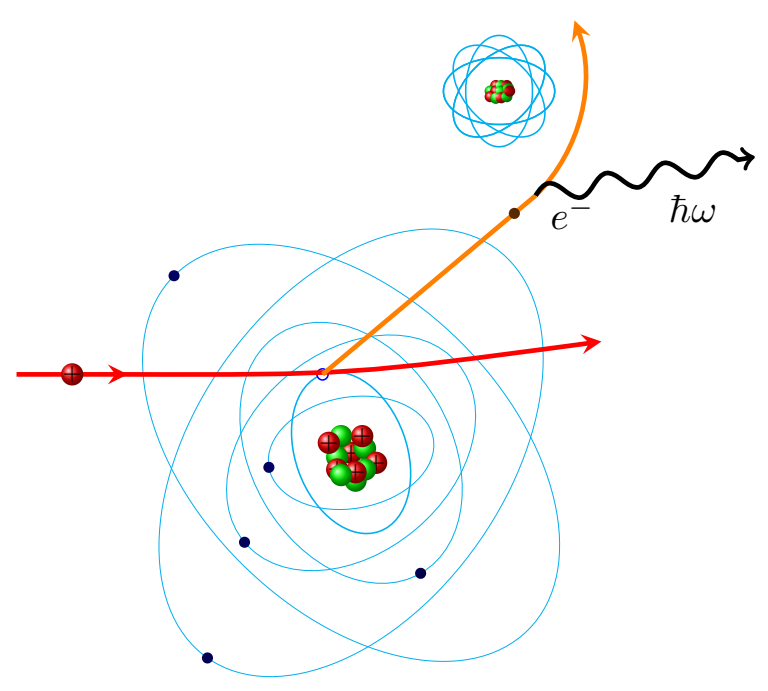

Figura 2.2: Schematic representation of the secondary electron bremsstrahlung (SEB) process.

More precisely, in the SEB process, the impinging proton, energy $E_{\mathrm{p}}$, ejects an electron from a target atom with energy $E_{\mathrm{e}}$ in the direction $\hat{e}$, making an angle $\theta_{\mathrm{e}}$ relative to the direction of the beam. Afterwards, the ejected electron loses energy down to $E_{\mathrm{e}}^{\prime}$ while passing through the medium. It thus produces bremsstrahlung in the Coulomb field of another nucleus.

The photon is emitted in the direction $\hat{k}$, which makes the angles $\theta_{\mathrm{br}}$, relative to $\hat{e}$, and $\theta$, with respect to the beam, assumed to be in the $z$ direction. Figure 2.3 illustrates the angles relating the directions of the ejected electron, the emitted photon, and the incident beam. As can be deduced from the figure, these angles are related by the expression

$$
\sin ^{2} \theta_{\mathrm{br}}=1-\left(\cos \theta_{\mathrm{e}} \cos \theta+\sin \theta_{\mathrm{e}} \sin \theta \cos \varphi_{\mathrm{e}}\right)^{2}
$$

Therefore, the SEB DDCS for an impinging proton of energy $E_{\mathrm{p}}$ can be generally expressed by (Ishii et al., 1976)

$$
\begin{aligned}
\frac{\mathrm{d}^{2} \sigma^{\mathrm{SEB}}}{\mathrm{d}(\hbar \omega) \mathrm{d} \Omega} & =\mathcal{N} \sum_{i} \int_{\hbar \omega}^{E_{\mathrm{p}}-U_{i}} \mathrm{~d} E_{\mathrm{e}} \int \mathrm{d} \Omega_{\mathrm{e}} \frac{\mathrm{d}^{2} \sigma_{i}^{\text {ion }}\left(E_{\mathrm{p}}, E_{\mathrm{e}}, \theta_{\mathrm{e}}\right)}{\mathrm{d} E_{\mathrm{e}} \mathrm{d} \Omega_{\mathrm{e}}} \\
& \times \int_{\hbar \omega}^{E_{\mathrm{e}}} \frac{\mathrm{d} E_{\mathrm{e}}^{\prime}}{S_{\mathrm{cs}}\left(E_{\mathrm{e}}^{\prime}\right)} \frac{\mathrm{d}^{2} \sigma^{\mathrm{br}}\left(E_{\mathrm{e}}^{\prime}, \hbar \omega, \theta_{\mathrm{br}}\right)}{\mathrm{d}(\hbar \omega) \mathrm{d} \Omega_{\mathrm{br}}},
\end{aligned}
$$

where $U_{i}$ is the binding energy of the $i$-th atomic subshell, $\mathcal{N}$ is the number of atoms per unit volume of the target material, $\mathrm{d}^{2} \sigma_{i}^{\text {ion }} / \mathrm{d} E_{\mathrm{e}} \mathrm{d} \Omega_{\mathrm{e}}$ is the ionization DDCS of the $i$-th subshell by proton impact, $S_{\mathrm{cs}}\left(E_{\mathrm{e}}^{\prime}\right)$ is the stopping cross section of the ejected electron, and $\mathrm{d}^{2} \sigma^{\mathrm{br}} / \mathrm{d}(\hbar \omega) \mathrm{d} \Omega_{\mathrm{br}}$ is the electron bremsstrahlung DDCS. 
Figura 2.3: Relation between the angles in the SEB DDCS. The direction of the incident beam is chosen to be $\hat{z}$, while $\hat{e}$ and $\hat{k}$ are the directions of the ejected electron and photon emission, respectively. Then, the angles are related by Equation (2.3.1).

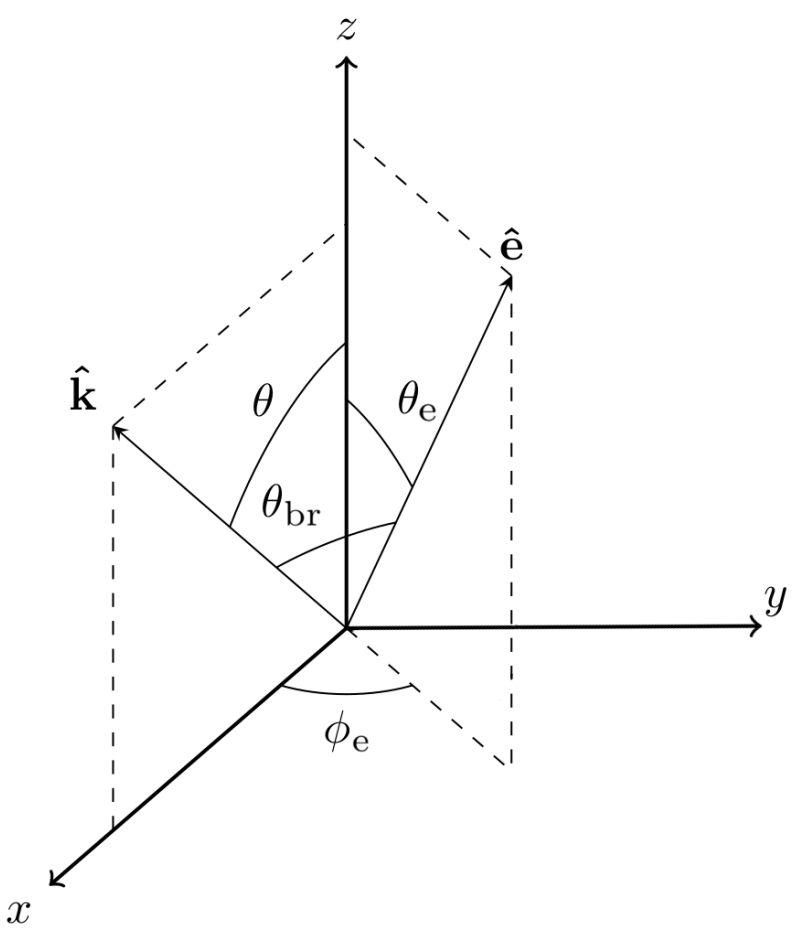

The ionization cross section, $\mathrm{d}^{2} \sigma_{i}^{\text {ion }} / \mathrm{d} E_{\mathrm{e}} \mathrm{d} \Omega_{\mathrm{e}}$, as described by Folkmann et al. (1974) and Ishii et al. (1976), is calculated in the binary encounter approximation (BEA) (Bonsen and Vriens, 1970; Folkmann et al., 1975). The BEA provides a DDCS for the ejection of an electron, from a given subshell of the target atom, and depends on the probability density function (PDF) of the speed of the electron, abbreviated as VD (velocity distribution) in the present work. It is therefore expected that the model used for the VD of the electrons must affect the ionization DDCS in the BEA (Mukoyama, 2015, 2018). In this sense, the analytical expression of the 1s hydrogenic VD has been used in all the literature published previously to the present work for the calculation of the proton bremsstrahlung processes (Ishii, 2006). These subjects will be discussed in more details in Section 2.3.1.1, for the ionization cross section, and Section 2.3.1.2, for the VD of the electrons.

In the second step of the SEB process, the ejected electron loses energy from $E_{\mathrm{e}}$ to $E_{\mathrm{e}}^{\prime}$ as it passes through the material. The expression for energy loss of the electron, $S\left(E_{\mathrm{e}}^{\prime}\right)$, can be calculated using the Bethe formula, as reviewed in Section 2.3.1.3. However, Ishii et al. (1976) present an approximation for that formula, whose validity is studied in Section 3.1.3.

Finally, the electron with energy $E_{\mathrm{e}}^{\prime}$ produces bremsstrahlung in the Coulomb field of another target nucleus. The bremsstrahlung production cross section for an electron of energy $E_{\mathrm{e}}^{\prime}, \mathrm{d}^{2} \sigma^{\mathrm{br}} / \mathrm{d}(\hbar \omega) \mathrm{d} \Omega_{\mathrm{br}}$, where $\hbar \omega$ and $\Omega_{\mathrm{br}}$ are the energy and the emission angle of the photon, can be expressed by Equation (2.2.2), if the result from the PWBA without screening is taken in the nonrelativistic limit. Ishii et al. (1976) further simplify Equation (2.2.2) using it in the 
low energy limit $\hbar \omega \ll E_{\mathrm{e}}^{\prime}$. This approximation for the electron-bremsstrahlung cross section results in

$$
\frac{\mathrm{d}^{2} \sigma^{\mathrm{br}}\left(E_{\mathrm{e}}^{\prime}, \hbar \omega, \theta_{\mathrm{br}}\right)}{\mathrm{d}(\hbar \omega) \mathrm{d} \Omega_{\mathrm{br}}}=\frac{2}{\pi} Z^{2} \alpha r_{0}^{2} \frac{1}{\beta_{\mathrm{e}}^{\prime 2}} \frac{1}{\hbar \omega} \ln \left(4 \frac{E_{\mathrm{e}}^{\prime}}{\hbar \omega}\right) \sin ^{2} \theta_{\mathrm{br}},
$$

where $\beta_{\mathrm{e}}^{\prime}=\left(2 E_{\mathrm{e}}^{\prime} / m_{\mathrm{e}} c^{2}\right)^{1 / 2}$. The validity of Equation (2.3.3) will be analyzed in Section 3.1.3.

It can be noted that the calculation of Equation (2.3.2) requires the evaluation of a $5 \mathrm{D}$ integral. Namely, the integrations are performed in the variables $\mathrm{d} E_{\mathrm{e}}, \mathrm{d} \theta_{\mathrm{e}}, \mathrm{d} \varphi_{\mathrm{e}}, \mathrm{d} v$ (see Section 2.3.1.1), and $d E_{\mathrm{e}}^{\prime}$. Nonetheless, a later work by Yamadera et al. (1981) presents a more explicit expression of the SEB DDCS, as an evolution of the formalism introduced in an earlier work (Ishii et al., 1976). The main advantage of the former approach by Yamadera et al. (1981) is that all integrals are performed analytically except the final one on the VD.

However, discrepancies appear when comparing results from the methods by (Ishii et al., 1976) and Yamadera et al. (1981) (see Section 3.3.2), besides differences with the formulae stated in the review article by Ishii (2006). It is important to note that these problems might have occurred due to typographical errors, considering the complexity of the equations involved.

To address this problem, an expression of the SEB DDCS in terms of an exponential integral function is proposed in the present work. With this approach, the numerical integration is reduced from 5D to 3D and the results are shown to agree with those by Ishii et al. (1976). This new approach will be discussed in Section 3.1, along with the implementation of the DHFS VDs in the calculation of the ionization DDCS in the BEA.

In this Section, the analytical expressions obtained by Yamadera et al. (1981) in terms of the BEA are presented. In this approach, the SEB DDCS is expressed as follows

$$
\frac{\mathrm{d}^{2} \sigma^{\mathrm{SEB}}}{\mathrm{d}(\hbar \omega) \mathrm{d} \Omega}=\sum_{i} N_{i} \frac{Z Z_{\mathrm{p}}^{2} a_{0}^{2}}{2 \pi}\left(\frac{e^{2}}{\hbar c}\right)^{5} \frac{m_{\mathrm{e}} c^{2}}{(\hbar \omega)^{2}}\left(C_{1 i}+C_{2 i} \sin ^{2} \theta\right)
$$

with

$$
\begin{aligned}
C_{1 i} & =\int_{\hbar \omega}^{\infty} \mathrm{d} E_{\mathrm{e}} \int_{0}^{\infty} f_{i}(v) \mathrm{d} v h_{1 i}\left(E_{\mathrm{e}}, v, U_{i}\right) \\
C_{2 i} & =\int_{\hbar \omega}^{\infty} \mathrm{d} E_{\mathrm{e}} \int_{0}^{\infty} f_{i}(v) \mathrm{d} v h_{2 i}\left(E_{\mathrm{e}}, v, U_{i}\right)
\end{aligned}
$$




$$
\begin{aligned}
& h_{1 i}\left(E_{\mathrm{e}}, v, U_{i}\right)=\frac{v_{i}}{v} \frac{g_{0}\left(3 f_{1} / 4-f_{2} / 4+f_{3} / 2\right)+g_{2}\left(-f_{1} / 4+3 f_{2} / 4-3 f_{3} / 2\right)}{\hbar \omega(E / \hbar \omega)^{3}} \\
& h_{2 i}\left(E_{\mathrm{e}}, v, U_{i}\right)=\frac{v_{i}}{v} \frac{g_{0}\left(-f_{1} / 8+3 f_{2} / 8-3 f_{3} / 4\right)+g_{2}\left(3 f_{1} / 8-9 f_{2} / 8+9 f_{3} / 4\right)}{\hbar \omega(E / \hbar \omega)^{3}} \\
& f_{1}=\int_{1}^{E_{\mathrm{e}} / \hbar \omega} \mathrm{d} t \frac{\ln [(1+T) /(1-T)]}{\ln [(1.16 \hbar \omega / \bar{I}) t]} \\
& f_{2}=\int_{1}^{E_{\mathrm{e}} / \hbar \omega} \mathrm{d} t \frac{\ln [(1+T) /(1-T)]}{\ln [(1.16 \hbar \omega / \bar{I}) t]} T^{2} \\
& f_{3}=\int_{1}^{E_{\mathrm{e}} / \hbar \omega} \mathrm{d} t \frac{T}{\ln [(1.16 \hbar \omega / \bar{I}) t]} \\
& g_{0}= \begin{cases}\frac{1}{6 x}\left[8+(s-x)^{3}\right] & \text { for } \quad\left|\frac{\gamma}{4}-1\right| \leq s \leq \infty, \\
\frac{s}{3 x}\left[s^{2}+3 x^{2}\right] & \text { for } \quad 1-\frac{\gamma}{4} \geq s \geq 0, \\
0 & \text { for } \quad s \leq \frac{\gamma}{4}-1 .\end{cases}
\end{aligned}
$$




$$
g_{2}= \begin{cases}\frac{\gamma}{2 x}\left[1-\left(\frac{\gamma-2 s}{2 x}\right)^{2}\right]+\frac{1}{7 \cdot 6 \cdot 5 \cdot 4 x^{3}} & \\ \times\left\{5 \cdot 4 \cdot 3\left[(1+s)(\gamma-2 s)^{3}-(x-1)(2 x)^{3}\right]\right. & \\ +9\left(\gamma+8 s^{2}+8\right)\left[(1+s)(\gamma-2 s)^{2}-(x-1)(2 x)^{2}\right] & \text { for }\left|\frac{\gamma}{4}-1\right| \leq s \leq \infty, \\ +4\left(8 \gamma-3 \gamma^{2}-27 \gamma s^{2}+22 s^{2}-24 s^{4}-24\right) & \\ \left.\times\left[2(x-1)\left(x^{2}+x+1\right)-(1+s)\left(2 x^{2}+2-2 s+\gamma\right)\right]\right\} & \\ \frac{s}{x^{3}}\left[\frac{\gamma^{3}}{4}+\left(\gamma^{2}+\frac{\gamma}{3}\right) s^{2}+\left(\frac{14}{15}+\frac{6 \gamma}{5}\right) s^{4}+\frac{16}{35} s^{6}\right] & \text { for } 1-\frac{\gamma}{4} \geq s \geq 0, \\ 0 & \text { for } s \leq \frac{\gamma}{4}-1,\end{cases}
$$

where the auxiliary variables are defined as

$$
\begin{aligned}
& \mathcal{E}=E_{\mathrm{e}}+U_{i}, \\
& T_{\mathrm{r}}=m_{\mathrm{e}} v_{\mathrm{p}}^{2} / 2=\left(m_{\mathrm{e}} / m_{\mathrm{p}}\right) E_{\mathrm{p}}, \\
& \gamma=\mathcal{E} / T_{\mathrm{r}}, \\
& s=v / v_{\mathrm{p}}, \\
& x^{2}=s^{2}+\gamma, \\
& T=\sqrt{1-1 / t} .
\end{aligned}
$$

In the above equations,

$\mathcal{E}$ is the energy transferred from the projectile to the electron;

$U_{i}=m_{\mathrm{e}} v_{i}^{2} / 2$ is the binding energy of the $i$-th shell;

$T_{\mathrm{r}}$ is the relative kinetic energy of the electron, i.e. the kinetic energy in the center-of-mass reference frame;

$v$ is the speed of the orbital electron in the target material;

$t$ is the energy of the electron (in units of $\hbar \omega$ ) as it passes though the target material; $f_{i}(v)$ is the VD of the electrons in the $i$-th subshell; and $N_{i}$ is the number of electrons in the $i$-th subshell. 


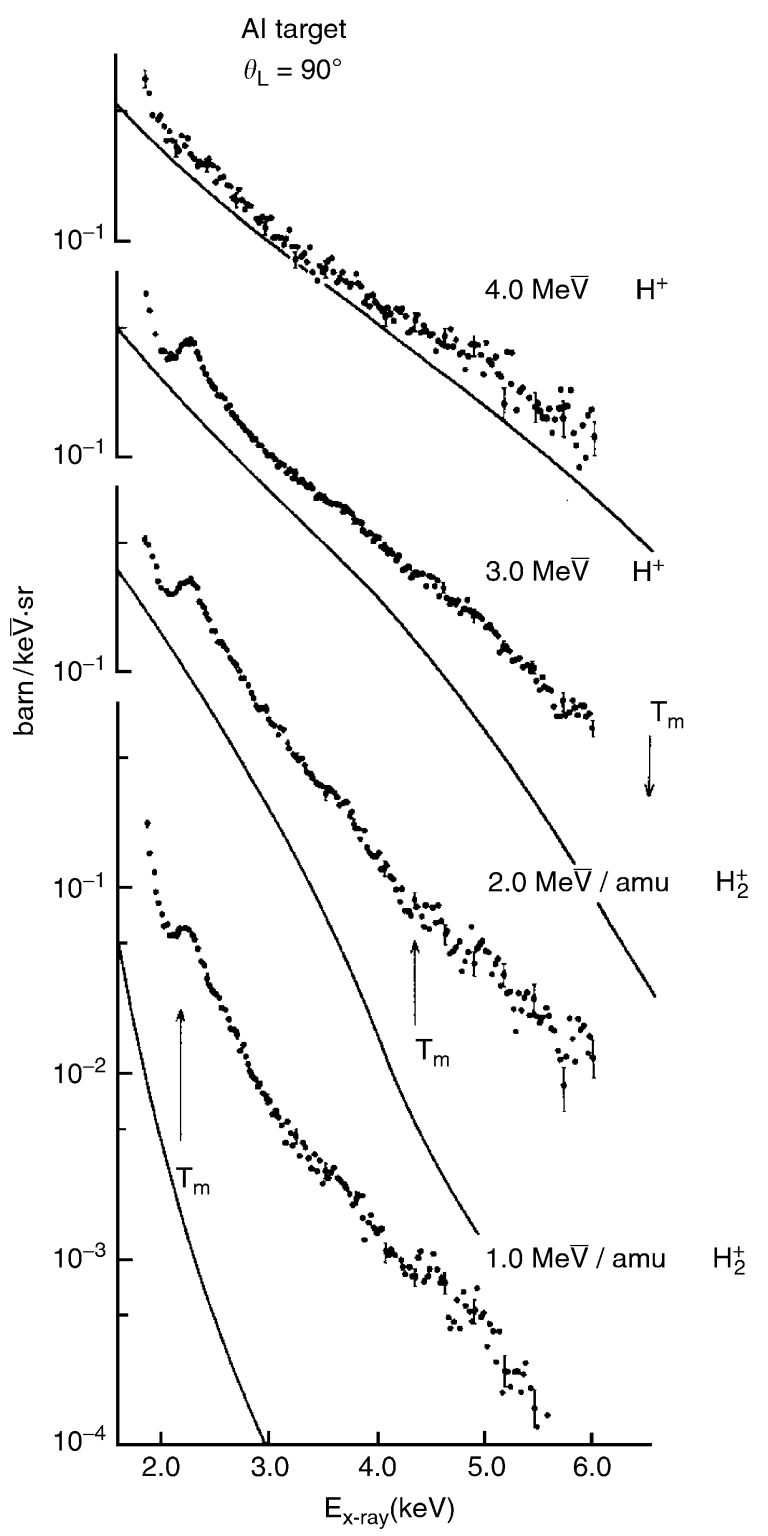

Figura 2.4: Comparison between theoretical (Equation (2.3.4)) SEB cross section and experimental results for an aluminum target, with 3 and $4 \mathrm{MeV}$ proton beams. The two lower graphs also show the results for 1 and $2 \mathrm{MeV} / \mathrm{amu} \mathrm{H}_{2}^{+}$beams. Source: Ishii and Morita (1990).

Moreover, in the BEA theory, the electrons are considered to be free, whilst the binding energy of the shell establishes its initial velocity. The maximum energy $T_{\max }$ transferred from the projectile of initial kinetic energy $E_{\mathrm{p}}$ to a free electron initially at rest is given by

$$
T_{\max }=\frac{4 m_{\mathrm{p}} m_{\mathrm{e}}}{\left(m_{\mathrm{p}}+m_{\mathrm{e}}\right)^{2}} E_{\mathrm{p}} \simeq 4 \frac{m_{\mathrm{e}}}{m_{\mathrm{p}}} E_{\mathrm{p}} .
$$

Consequently, $T_{\max }$ also defines the maximum energy of the photon that a secondary electron can emit. Therefore, the SEB cross section is characterized by the quantity $T_{\max }$. 
Nonetheless, the VD of the electrons introduces a tail beyond the energy $T_{\max }$ in the SEB DDCS. At low photon energies, where $\hbar \omega<T_{\max }$, the SEB contribution to the spectrum is most important, but it rapidly decreases at energies above $T_{\max }$, as can be seen in Figure 2.4 As a consequence, the SEB DDCS contributes more to the experimental data with increasing energies of the projectile, i.e. higher $T_{\max }$ values. Above $T_{\max }$, however, the SEB DDCS stays well below the experimental data, whereas the $A B$ process is predominant.

\subsubsection{Ionization by impact of a proton}

There are several theoretical models for the calculation of the ionization by impact of a proton, such as the Binary Encounter Approximation (BEA), the Plane Wave Born Approximation (PWBA), and its improvement, the ECPSSR theory, among others.

In the BEA theory, the collision between the projectile (proton) and the scattering center (electron) is treated in nonrelativistic quantum mechanics, and the cross section is calculated in terms of the exchanged momenta, applying the energy and momentum conservation laws of classical mechanics (Bonsen and Vriens, 1970; Folkmann et al., 1975). The target electrons are assumed to be free, for which the atomic nucleus establishes the initial momentum distribution. The binding energy is however taken into account in the energy conservation. Therefore, the $\mathrm{VD}$ of the electron is of fundamental importance in the calculation of the ionization process in the BEA.

In the PWBA approach, the initial state consists of a plane wave for the proton and a bound state wave function for the electron, while the final state assumes a plane wave for the deflected proton and the ejected electron. Then, perturbation theory is used to describe the transition between the initial and the final states submitted to Coulomb interactions. Both BEA and PWBA theories present good results for proton energies much greater than the binding energy of the electron in the atom. They reproduce important aspects of the experimental results.

Lastly, in order to refine the results and improve the agreement with the experimental results, a series of modifications were implemented to the PWBA theory resulting in the ECPSSR theory, whose acronym refers to each theoretical correction introduced in the formulation. Accordingly, the ECPSSR theory accounts for energy loss during the collision (E), deflection and velocity change of the projectile due to Coulomb interaction $(\mathrm{C})$, perturbation of the atomic stationary states by the projectile (PSS) and relativistic effects (R) (Brandt and Lapicki, 1979). These improvements are treated as an effective energy of the projectile and an effective binding energy of the target electron. This method of calculation of the ionization cross section presents excellent agreement with experimental data, specially for the K-shell (Cohen and Harrigan, 1985). 
As mentioned above, calculations of the SEB DDCS were performed by Folkmann et al. (1974) and Ishii et al. (1976) by considering the BEA as an approach to the ionization DDCS. In the SEB DDCS, Equation (2.3.2), the ionization DDCS is given in the BEA by (Bonsen and Vriens, 1970)

$$
\frac{\mathrm{d}^{2} \sigma_{i}^{\text {ion }}\left(E_{\mathrm{p}} ; E_{\mathrm{e}}, \theta_{\mathrm{e}}\right)}{\mathrm{d} E_{\mathrm{e}} \mathrm{d} \Omega_{\mathrm{e}}}=N_{i} \int_{v^{\min }\left(E_{\mathrm{p}} ; E_{\mathrm{e}}, \theta_{\mathrm{e}}\right)}^{v^{\max }\left(E_{\mathrm{p}} ; E_{\mathrm{e}}, \theta_{\mathrm{e}}\right)} \frac{\mathrm{d}^{2} \sigma^{\mathrm{e}}\left(E_{\mathrm{p}}, v ; E_{\mathrm{e}}, \theta_{\mathrm{e}}\right)}{\mathrm{d} E_{\mathrm{e}} \mathrm{d} \Omega_{\mathrm{e}}} f_{i}(v) \mathrm{d} v,
$$

where the limits of integration $v^{\min }$ and $v^{\max }$ are defined by applying the laws of momentum and energy conservation in the scattering process, as described by Bonsen and Vriens (1970). Moreover, the term $\mathrm{d}^{2} \sigma^{\mathrm{e}} / \mathrm{d} E_{\mathrm{e}} \mathrm{d} \Omega_{\mathrm{e}}$ is the DDCS for ejection of an electron by impact of a proton with energy $E_{\mathrm{p}}$. The DDCS for ejection of an electron is derived from the Rutherford scattering cross section in terms of the energy transfer $\mathcal{E}$, and it is expressed by (Bonsen and Vriens, 1970; Folkmann et al., 1974)

$$
\begin{aligned}
& \frac{\mathrm{d}^{2} \sigma^{\mathrm{e}}}{\mathrm{d} E_{\mathrm{e}} \mathrm{d} \Omega_{\mathrm{e}}}\left(E_{\mathrm{p}}, v ; E_{\mathrm{e}}, \theta_{\mathrm{e}}\right)=\frac{r_{\mathrm{e}}^{2} m_{\mathrm{e}} c^{2}}{2} \frac{v_{\mathrm{p}} v_{\mathrm{e}}}{v} \frac{1}{\mathcal{E}^{3}}\left\{m_{\mathrm{e}} c^{2} v_{\mathrm{e}}^{2} \sin ^{2} \theta_{\mathrm{e}}\right. \\
& \left.\quad-\mathcal{E}\left[1-\left(1+\frac{m_{\mathrm{e}}}{m_{\mathrm{p}}}\right) \frac{v_{\mathrm{e}}}{v_{\mathrm{p}}} \cos \theta_{\mathrm{e}}+\frac{m_{\mathrm{e}}}{m_{\mathrm{p}}} \frac{v_{\mathrm{e}}^{2}}{v_{\mathrm{p}}^{2}}\right]\right\}\left(v_{\mathrm{p}}^{2}+v_{\mathrm{e}}^{2}-2 v_{\mathrm{p}} v_{\mathrm{e}} \cos \theta_{\mathrm{e}}\right)^{-3 / 2},
\end{aligned}
$$

where $E_{e}=\mathcal{E}-U_{i}$ is the kinetic energy of the ejected electron, according to Equation (2.3.9a), and $\beta_{e}=\left(\beta^{2}+2 \mathcal{E} / m_{\mathrm{e}} c^{2}\right)^{1 / 2}$ is the velocity of the ejected electron.

The ionization DDCS in the BEA is defined for each subshell of the target atom separately. This means that the integration is performed for each subshell with its corresponding VD of the electrons, $f_{i}(v)$, which is an important aspect of the BEA. In the case of the protoninduced bremsstrahlung processes discussed here, and particularly the SEB mechanism, it can be noted that published works generally make use of the 1s hydrogenic PDF for all subshells (Ishii, 2006).

Figure 2.5 shows the results for Equation (2.3.12) obtained numerically by Bonsen and Vriens (1970). Each curve was calculated for a fixed energy of the ejected electron and uses the 1s hydrogenic VD of the orbital electrons. This data served as a reference for the validation of the implementation of Equation (2.3.12) in the calculations of secondary electron bremsstrahlung (see Section 3.2). In that same paper, the authors also provide a graph for the energy distribution of ejected electrons at a fixed angle in comparison to experimental data (see Fig. 5 in (Bonsen and Vriens, 1970)), which shows a good agreement for energies above $300 \mathrm{eV}$. 


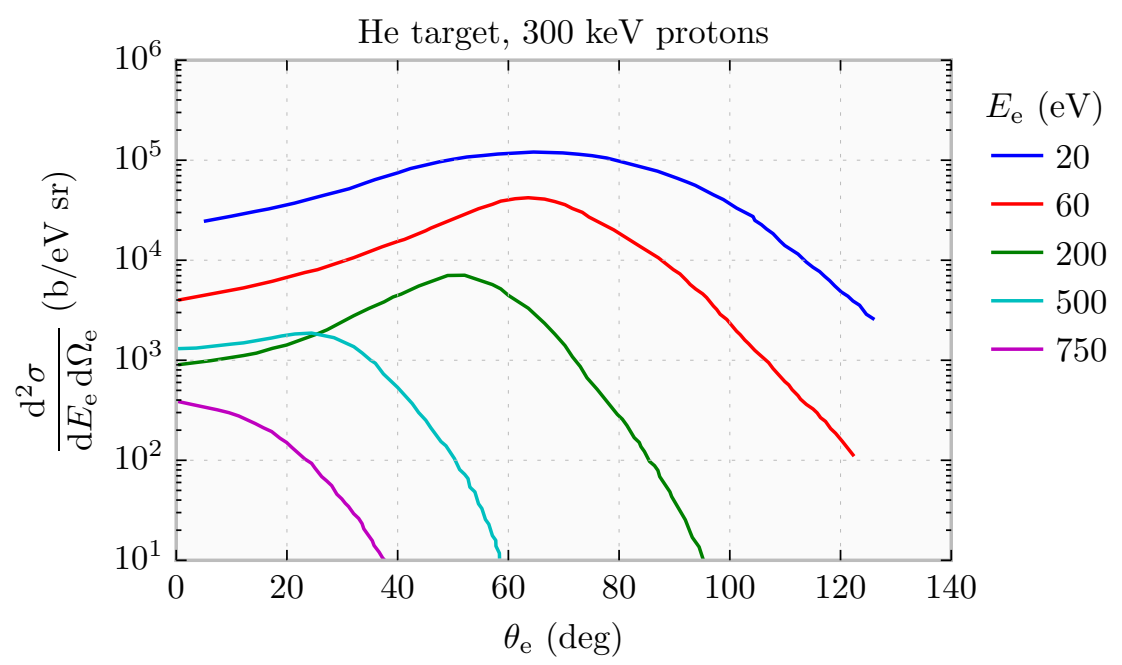

Figura 2.5: Ionization cross section by proton impact as a function of the electron ejection angle, for various energies of the ejected electron. The case considered is that of a He target bombarded with $300 \mathrm{keV}$ protons. Source: Bonsen and Vriens (1970).

\subsubsection{Velocity distribution of the electrons}

As mentioned above, current calculations of the SEB DDCS rely on the ionization DDCS in the BEA and are usually performed assuming that the VD of the electrons is provided by the hydrogenic 1s wave function for all shells. This is an important approximation, since it was shown that the ionization DDCS in the BEA is sensitive to the choice of the VD model (Mukoyama, 2015, 2018). However, as the atomic number of the target element increases, the hydrogenic VD cannot account for the screening effects in the outer shells, as the potential felt by the electrons is non Coulombian, and the relativistic effects in the inner shells, which have higher values of the binding energy. To approach these issues, use of numerical calculations of the VDs from the DHFS method is proposed in Chapter 3.

\section{Nonrelativistic wave functions}

The VD of the orbital electrons can be derived from the wave function in momentum space, $\Phi(\boldsymbol{p}, t)$, which is the Fourier transform of the wave function in position space, $\psi(\boldsymbol{r}, t)$. In particular, the simple case of the Hydrogen atom allows for an analytical expression of the 1s wave function $\psi_{1 s}(r)$, which is a solution of the time-independent Schrödinger equation. Therefore, the hydrogenic $1 \mathrm{~s}$ wave function in momentum space is given by

$$
\phi_{1 s}(\boldsymbol{p})=\frac{1}{(2 \pi \hbar)^{3 / 2}} \int e^{-i(\boldsymbol{p} \cdot \boldsymbol{r}) / \hbar} \psi_{1 s}(\boldsymbol{r}) \mathrm{d}^{3} \boldsymbol{r}=\frac{2^{3 / 2}}{\pi} \frac{p_{0}^{5 / 2}}{\left[p_{0}^{2}+p^{2}\right]^{2}}
$$


where $p_{0}=\hbar / a_{0}$, with $a_{0}=\hbar^{2} / m_{\mathrm{e}} e^{2}$ the Bohr radius. Since the wave function $\phi_{1 s}(\boldsymbol{p})$ is normalized to unity, the PDF in momentum space is obtained from

$$
f_{1 s}(p)=\left|\phi_{1 s}(\boldsymbol{p})\right|^{2} p^{2} 4 \pi=\frac{32}{\pi} \frac{p_{0}^{5} p^{2}}{\left[p_{0}^{2}+p^{2}\right]^{4}}
$$

For the VD of the electron, a transformation of variable shows

$$
\int_{0}^{\infty} f_{1 s}(p) \mathrm{d} p=\int_{0}^{\infty} f_{1 s}(m v) \frac{\mathrm{d} p}{\mathrm{~d} v} \mathrm{~d} v=1 \quad \Rightarrow \quad f_{1 s}(v)=\frac{32}{\pi} \frac{v_{0}^{5} v^{2}}{\left[v_{0}^{2}+v^{2}\right]^{4}}
$$

Another approach to obtain the momentum space wave function is to rewrite the Schrödinger equation in momentum space and explicitly solve it for $\phi(p)$. The method is described by Bethe and Salpeter (1957) and consists in expressing the Schrödinger equation as an integral equation in momentum space. Then, the explicit solution for the radial momentum space wave functions $F_{n \ell}(p)$ of the hydrogen atom is given by (Bethe and Salpeter, 1957)

$$
F_{n \ell}(p)=\left[\frac{2}{\pi} \frac{(n-\ell-1) !}{(n+\ell) !}\right]^{\frac{1}{2}} n^{2} 2^{2(\ell+1)} \ell ! \frac{n^{\ell} p^{\ell}}{\left(n^{2} p^{2}+1\right)^{\ell+2}} C_{n-\ell-1}^{\ell+1}\left(\frac{n^{2} p^{2}-1}{n^{2} p^{2}+1}\right)
$$

where $n$ and $\ell$ are the principal and the orbital angular momentum quantum numbers, respectively; the momentum $p$ is expressed in units of $p_{0}=\hbar / a_{0}$; and $C_{N}^{v}(x)$ is the Gegenbauer function. For $N=0,1,2$, the corresponding expressions for $C_{N}^{v}(x)$ are given by

$$
\begin{aligned}
& C_{0}^{v}(x)=1, \\
& C_{1}^{v}(x)=2 v x, \\
& C_{2}^{v}(x)=2 v(v+1) x^{2}-v .
\end{aligned}
$$

Thus with quantum numbers $n=1,2$, and $\ell=0,1$, Equation (2.3.16) results in the first three radial wave functions in momentum space

$$
\begin{aligned}
& F_{10}=4 \sqrt{\frac{2}{\pi}} \frac{1}{\left(p^{2}+1\right)^{2}}, \\
& F_{20}=\frac{32}{\sqrt{\pi}} \frac{4 p^{2}-1}{\left(4 p^{2}+1\right)^{3}}, \\
& F_{21}=\frac{128}{\sqrt{3 \pi}} \frac{p}{\left(4 p^{2}+1\right)^{3}} .
\end{aligned}
$$


The radial wave functions expressed by Equation (2.3.16) are normalized to unity

$$
\int_{0}^{\infty}\left|F_{n \ell}(p)\right|^{2} p^{2} \mathrm{~d} p=1
$$

from which the VD of the electron can be obtained. It can be verified that the particular case of Equation (2.3.18a) matches the result (2.3.14) obtained via Fourier transform of the corresponding position space wave function, noting that the former expresses momentum $p$ in units of $\hbar / a_{0}$. Moreover, Equation (2.3.16) can be extended for other values of the atomic number $Z$ by substituting $p$ by $Z p_{0}$ (Bethe and Salpeter, 1957).

\section{Relativistic wave functions}

When considering higher values of the atomic number $\mathrm{Z}$, relativistic effects can no longer be neglected, because the binding energy greatly increases for the inner shells of atoms with a larger $Z$. Moreover, in these cases, screening effects in the outer shells must also be taken into account. However, the analytical expression of the hydrogenic PDF, Equation (2.3.14), assumes that the electron interacts with a pure Coulomb potential. In this sense, numerical PDFs obtained from the DHFS method provide more consistent expressions accounting for those two aspects.

The relativistic wave equation, known as the Dirac equation, for an electron in a central potential $V(r)$ is given by (Liberman et al., 1965; Salvat and Fernández-Varea, 2019)

$$
\left[c \boldsymbol{\alpha} \cdot \boldsymbol{p}+(\beta-1) m_{\mathrm{e}} c^{2}+V(r)\right] \psi_{n \kappa m}(\boldsymbol{r})=E_{n \kappa} \psi_{n \kappa m}(\boldsymbol{r})
$$

where $\boldsymbol{p}=-i \hbar \nabla$ is the momentum operator; $E_{n \kappa}$ is the kinetic energy eigenvalue (i.e. without the rest energy contribution); $n, \kappa$, and $m$ are the principal, the relativistic angular momentum, and magnetic quantum numbers; $\boldsymbol{\alpha}$ and $\beta$ are $4 \times 4$ matrices, which can be represented in terms of the Pauli spin matrices and the $2 \times 2$ unit matrix $\mathbb{1}$ by (Bransden et al., 2000)

$$
\begin{aligned}
& \alpha=\left(\begin{array}{ll}
0 & \sigma \\
\sigma & 0
\end{array}\right), \\
& \beta=\left(\begin{array}{cc}
\mathbb{1} & 0 \\
0 & -\mathbb{1}
\end{array}\right) .
\end{aligned}
$$


The solutions $\psi_{n \kappa m}(\boldsymbol{r})$ of Equation (2.3.20) are given by the central-field orbitals

$$
\psi_{n \kappa m}(\boldsymbol{r})=\frac{1}{r}\left(\begin{array}{c}
P_{n \kappa}(r) \Omega_{\kappa, m}(\hat{\boldsymbol{r}}) \\
i Q_{n \kappa}(r) \Omega_{-\kappa, m}(\hat{\boldsymbol{r}})
\end{array}\right),
$$

where, as above, $\kappa$ and $m$ are the relativistic angular momentum and magnetic quantum numbers, respectively; $P_{n \kappa}(r)$ and $Q_{n \kappa}(r)$ are the large and small reduced radial wave functions; and $\Omega_{\kappa, m}(\hat{\boldsymbol{r}})$ are two-component spherical spinors.

The potential $V(r)$ in Equation (2.3.20) is given by (Salvat and Fernández-Varea, 2019)

$$
V(r)=V_{\mathrm{nuc}}(r)+V_{\mathrm{el}}(r)+V_{\mathrm{ex}}(r)
$$

where $V_{\text {nuc }}(r)$ is the nuclear potential, $V_{\mathrm{el}}(r)$ is the electronic potential, and $V_{\mathrm{ex}}(r)$ is the exchange potential. In the DHFS method, the multi-electron wave function is represented as a Slater determinant of the single electron wave functions of the form given in Equation (2.3.22). The multi-electron wave function is used to calculate the radially symmetric mean field associated to atomic electrons $V_{\mathrm{el}}$ and a self-consistent procedure is employed to achieve convergence. The exchange potential is modelled semi-empirically to simplify the numerical implementation. More details can be found in the work by Salvat and Fernández-Varea (2019).

The radial functions $P_{n \kappa}(r)$ and $Q_{n \kappa}(r)$ in the orbitals from Equation (2.3.22) satisfy the radial equations

$$
\begin{aligned}
& \frac{\mathrm{d} P_{n \kappa}}{\mathrm{d} r}=-\frac{\kappa}{r} P_{n \kappa}+\frac{E_{n \kappa}-V+2 m_{\mathrm{e}} c^{2}}{c \hbar} Q_{n \kappa} \\
& \frac{\mathrm{d} Q_{n \kappa}}{\mathrm{d} r}=-\frac{E_{n \kappa}-V}{c \hbar} P_{n \kappa}+\frac{\kappa}{r} Q_{n \kappa} .
\end{aligned}
$$

Equation (2.3.24) can be solved numerically for the reduced radial wave functions $P_{n \kappa}(r)$ and $Q_{n \kappa}(r)$. Hence, the corresponding wave functions in the momentum space can be obtained from the Fourier-Bessel transforms (omitting an irrelevant phase) (Segui et al., 2002)

$$
\begin{aligned}
& \widetilde{P}_{n \kappa}(p)=\hbar^{-3 / 2} \sqrt{\frac{2}{\pi}} \int_{0}^{\infty} j_{\ell}(p r / \hbar) P_{n \kappa}(r) r \mathrm{~d} r \\
& \widetilde{Q}_{n \kappa}(p)=\hbar^{-3 / 2} \sqrt{\frac{2}{\pi}} \int_{0}^{\infty} j_{\bar{\ell}}(p r / \hbar) Q_{n \kappa}(r) r \mathrm{~d} r
\end{aligned}
$$

where $j_{\ell}$ are the spherical Bessel functions; $\ell$ and $\bar{\ell} \equiv \ell-\frac{\kappa}{|\kappa|}$ are the orbital angular momenta corresponding to $\kappa$ and $-\kappa$, respectively. 
Finally, from $\widetilde{P}_{n \kappa}(p)$ and $\widetilde{Q}_{n \kappa}(p)$, the PDFs for the modulus of the linear momentum are given by

$$
f_{n \kappa}(p)=\left[\widetilde{P}_{n \kappa}^{2}(p)+\widetilde{Q}_{n \kappa}^{2}(p)\right] p^{2}
$$

which are normalized as

$$
\int_{0}^{\infty} f_{n \kappa}(p) \mathrm{d} p=1
$$

For the present work, the radial wave functions $P_{n \kappa}(r)$ and $Q_{n \kappa}(r)$ have been calculated by J. M. Fernández-Varea, using a program developed at the University of Barcelona (Salvat and Fernández-Varea, 2019). The VD have been calculated using Equation (2.3.27) from the numerical wave functions by J. M. Fernández-Varea. Finally, the DHFS VD have been used in the calculation of the ionization and the SEB DDCSs, in order to study the influence of the VD model in the SEB process. The results are discussed in Chapters 3 and 5.

\subsubsection{Energy loss}

When fast charged particles, such as electrons, protons, alpha particles, etc, pass through matter, they collide with atoms of the medium along their path, thus exciting or ionizing these atoms and losing energy. In this sense, the process of energy loss is also called ionization loss. Some of the incident particles undergo close collisions with the atoms of the target material and have their paths randomly deflected, thus they can also lose energy by bremsstrahlung. In the case of electrons, the energy loss by radiation emission is larger due to their relatively smaller mass and ample deflections. A critical energy of the incident electrons can be defined so that the energy loss due to bremsstrahlung surpasses the ionization loss. On the other hand, in the case of heavier particles, such as protons, the ionization loss is the predominant mechanism of energy loss.

The phenomenon of energy loss by a fast charged particle has important applications in experimental physics. For example, particles can be identified by the analysis of their paths in tracking detectors. The quantity of energy lost per distance traveled in the material depends on the particle velocity, so by measuring the energy loss and the momentum of a particle it is possible to determine its mass.

The theoretical formulation of energy loss per unit length, denoted by $\mathrm{d} E / \mathrm{d} x$, for nonrelativistic particles was described by Bethe (1930), and a relativistic version was derived by the same author in 1932. In both cases, the energy loss in each collision is properly treated by quantum mechanics. A simpler classical approach, however, can be discussed by considering 
Tabela 2.1: Mean excitation energy $\bar{I}$, in eV, for a few elements, used in the energy loss calculations. ${ }^{1}$

\begin{tabular}{|r|r|c|}
\hline $\mathrm{Z}$ & Element & $\bar{I}(\mathrm{eV})$ \\
\hline 13 & Aluminum & 166 \\
\hline 47 & Silver & 470 \\
\hline 79 & Gold & 790 \\
\hline
\end{tabular}

the energy transfer from an incident heavy ion, charge $Z_{p}$ and speed $\beta_{\mathrm{p}}$, to an essentially free and at rest electron. The particle gives an impulse to the electron in the direction perpendicular to its path (Jackson, 1999). The energy transfer depends on the impact parameter, $b$, and the integration over all possible values of $b$ results in the expression (Yuan and $\mathrm{Wu}, 1961$ )

$$
-\frac{\mathrm{d} E}{\mathrm{~d} x}=\frac{4 \pi \mathcal{N} Z Z_{p}^{2} e^{4}}{m_{\mathrm{e}} c^{2}} \frac{1}{\beta_{\mathrm{p}}^{2}} \ln \left(\frac{2 m_{\mathrm{e}} c^{2} \beta_{\mathrm{p}}^{2}}{\bar{I}\left(1-\beta_{\mathrm{p}}^{2}\right)}\right),
$$

where $\mathcal{N}, Z$, and $\bar{I}$ are the number of atoms per unit volume, the atomic number, and the mean excitation energy of the target material, respectively. The minus sign on the left hand side of the equation is due to the decrease in energy of the incident particle.

The mean excitation energy $\bar{I}$ is an atomic property fundamentally determined by experimental measurements of the energy loss. As a remark, some values of $\bar{I}$ are given in Table 2.1.

In addition to Equation (2.3.29), the total relativistic energy loss formula takes into account the density effect, being expressed by

$$
-\frac{\mathrm{d} E}{\mathrm{~d} x}=\frac{4 \pi N Z Z_{p}^{2} e^{4}}{m_{\mathrm{e}} c^{2}} \frac{1}{\beta_{\mathrm{p}}^{2}}\left[\ln \left(\frac{2 m_{\mathrm{e}} c^{2} \beta_{\mathrm{p}}^{2}}{\bar{I}\left(1-\beta_{\mathrm{p}}^{2}\right)}\right)-\beta_{\mathrm{p}}^{2}-\delta\right],
$$

where $\delta$ is the density effect, given by (Yuan and $\mathrm{Wu}, 1961$ )

$$
\delta=-\ln \left(1-\beta_{\mathrm{p}}^{2}\right)-2 \ln \left(\frac{\bar{I}}{\hbar \omega_{p}}\right)-1
$$

where $\omega_{p}^{2}=4 \pi N Z e^{2} / m_{\mathrm{e}}$ is the plasma frequency. The density effect was first treated by Fermi (1940) and occurs due to the polarization of the medium in dense materials, which alters the electromagnetic fields of the impinging particle. The values of free-space fields are no longer valid, and the characteristics of macroscopic fields in dielectrics must be considered.

However, in the calculations of the SEB process, Ishii et al. (1976) implemented an

\footnotetext{
${ }^{1}$ Source: https://physics.nist.gov/PhysRefData/XrayMassCoef/tab1.html. Accessed on March/2018.
} 


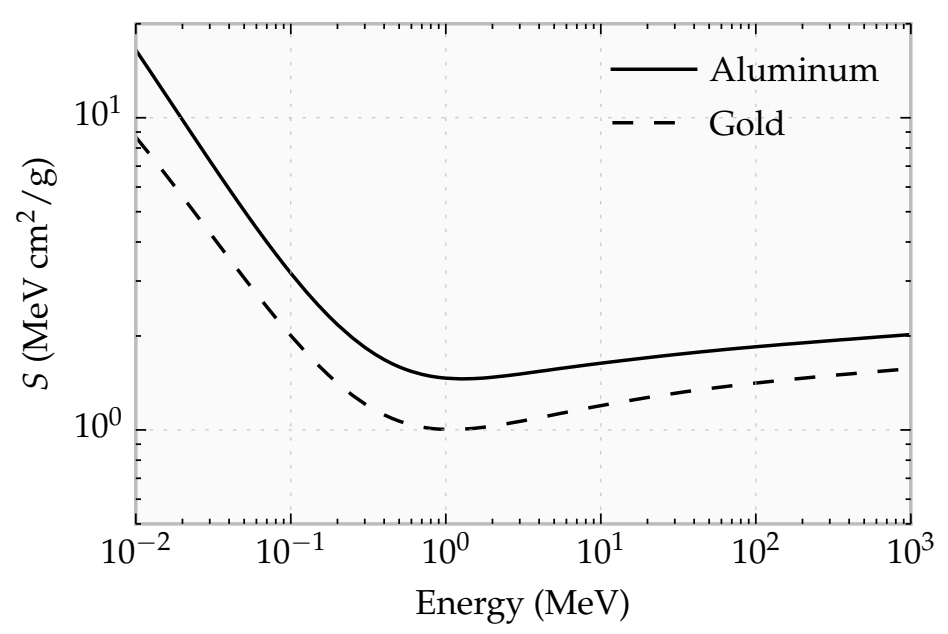

Figura 2.6: Reference values of the stopping power for electrons in aluminum and gold. Values retrieved from the NIST database ${ }^{2}$.

approximation to Equation (2.3.29) for an electron ejected with speed $\beta_{\mathrm{e}}$, given by

$$
-\frac{\mathrm{d} E}{\mathrm{~d} x}=\frac{4 \pi N Z e^{4}}{m_{\mathrm{e}} c^{2}} \frac{1}{\beta_{\mathrm{e}}^{2}} \ln \left(\frac{1.16}{\bar{I}} E_{\mathrm{e}}\right),
$$

with $E_{\mathrm{e}}=m_{\mathrm{e}} c^{2} \beta_{\mathrm{e}}^{2} / 2$. In Section 3.1.4, it will be shown that Equation (2.3.32) is a good approximation for small energies of the electron, mainly below $1 \mathrm{MeV}$.

Finally, the stopping force $S_{\mathrm{f}}(E)$ is defined as the retarding force acting on the particle and is numerically equal to the energy loss, that is

$$
S_{\mathrm{f}}(E)=-\frac{\mathrm{d} E}{\mathrm{~d} x}
$$

From that, the stopping power, $S(E)$, and the stopping cross section, $S_{\mathrm{cs}}$, can also be defined as $S(E)=S_{\mathrm{f}} / \rho$ and $S_{\mathrm{cs}}=S_{\mathrm{f}} / \mathcal{N}$, where $\rho$ and $\mathcal{N}$ are the mass and number density of the medium, respectively. Figure 2.6 shows the values of stopping power for electrons in aluminum and gold. These values were obtained from NIST online database (Berger et al., 2017).

The range $\Delta x$ of a particle traveling in a straight path in the material is thus calculated in the continuous slowing down approximation (CSDA) by

$$
\Delta x=\int_{0}^{E_{0}} \frac{1}{S_{\mathrm{f}}(E)} \mathrm{d} E
$$

where $E_{0}$ is the initial kinetic energy of the particle.

\footnotetext{
${ }^{2}$ https://www.nist.gov/pml/stopping-power-range-tables-electrons-protons-and-helium-ions. Accessed on March/2018.
} 


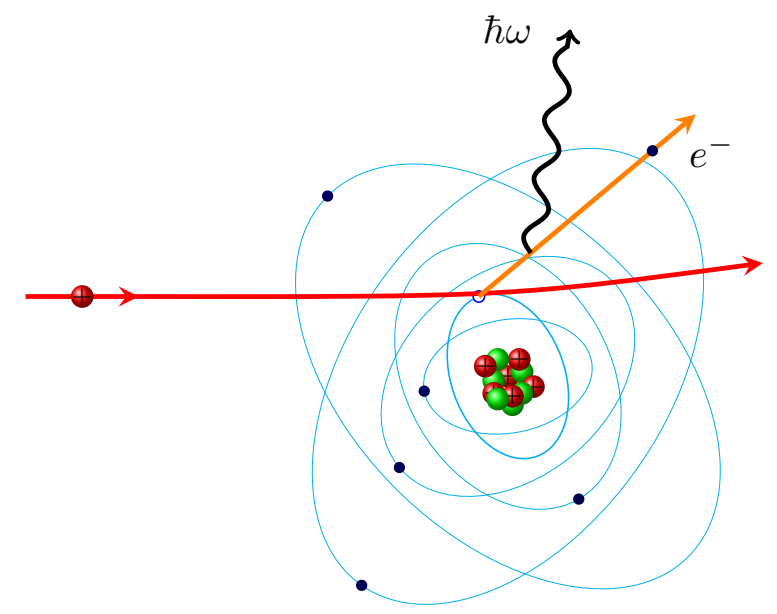

Figura 2.7: Schematic representation of the atomic bremsstrahlung $(\mathrm{AB})$ and radiative ionization (RI) processes (Ishii, 2006).

\subsubsection{Atomic Bremsstrahlung and Radiative Ionization}

In the Atomic Bremsstrahlung $(\mathrm{AB})$ process, the incident proton excites one of the electrons of the target atom to the continuum. The electron, bound by the field of the atom, produces bremsstrahlung when excited and, in this case, returns to its initial bound state. On the other hand, if the electron remains in the continuum, i.e. it is ionized, the bremsstrahlung process is called Radiative Ionization (RI), as illustrated in Figure 2.7.

The AB process was described in terms of the PWBA theory by Ishii and Morita (1984). They compared the results to experimental measurements from an aluminum target bombarded with 1 and $4 \mathrm{MeV}$ protons and for photons emitted at an angle of $90^{\circ}$ relative to the beam. The comparison showed good agreement in these cases; note that the atomic bremsstrahlung is more intense than other processes in the x-ray energy region of $\hbar \omega>T_{\max }, T_{\max }$ being the maximum energy that can be transferred from the projectile to a free electron at rest (see Equation (2.3.10)).

Later, Ishii et al. (2005) compared the predictions for $\mathrm{AB}$ within the PWBA theory for silver and gold targets and an incident beam of $1.5 \mathrm{MeV}$ protons, for which the results overestimated the experimental data by one order of magnitude. In the same work, this discrepancy was shown to be reduced by introducing the screening effect by the target electrons (Ishii et al., 2005).

However, the PWBA theory for $\mathrm{AB}$ does not fully agree with the experimental data (Ishii et al., 2008). In the case of an Ag target, the theoretical curve differs in shape from the experimental spectrum, as can be seen in the left panel of Figure 2.8. For the Au target, shown in the right panel of Figure 2.8, the theory still overestimates the experimental 
(a) Ag target

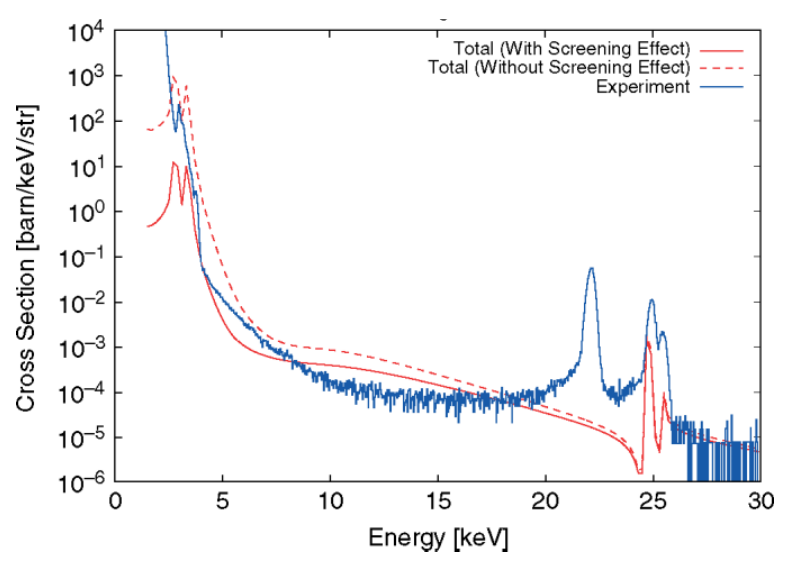

(b) Au target

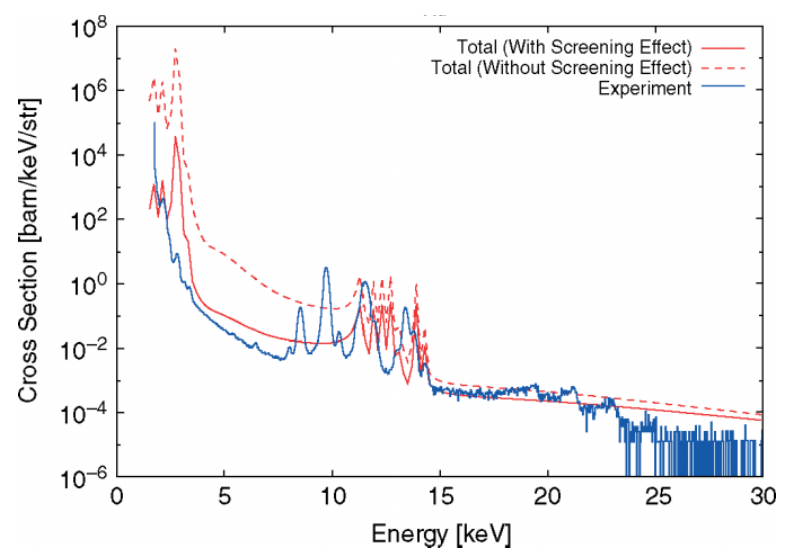

Figura 2.8: $\mathrm{AB}$ cross sections of (a) silver and (b) gold targets bombarded with $1.5 \mathrm{MeV}$ protons and measured at $90^{\circ}$. The solid (dashed) line shows the PWBA theory results with (without) screening effect. Source: Ishii et al. (2008).

measurements, since for the yields above $15 \mathrm{keV}$ the pile-up with Au L-x-rays must be taken into account.

Therefore, Ishii et al. (2008) calculated the AB cross section on the basis of the BEA approximation to compare with experimental data for the $\mathrm{Ag}$ and $\mathrm{Au}$ targets. In this approach, target electrons are assumed to be free and the ionization cross section, $\sigma_{i}^{\text {ion }}$, is given by

$$
\sigma_{i}^{\text {ion }}=\int_{0}^{\infty} \mathrm{d} v_{i} f_{i}(v) \int_{U_{i}}^{\infty} \mathrm{d} E \int \mathrm{d} q \sigma^{R},
$$

where $f_{i}(v)$ is the VD of the electrons, $U_{i}$ is the binding energy of the $i$-th subshell, $E$ and $q$ are the energy and momentum transfer between the projectile and the orbital electron, respectively, and $\sigma^{R}$ is the differential Rutherford cross section. On the other hand, the nonrelativistic formula for the electron bremsstrahlung cross section is expressed by (Ishii et al., 2008)

$$
\frac{\mathrm{d}^{2} \sigma^{\mathrm{elec}-\mathrm{br}}}{\mathrm{d}(\hbar \omega) \mathrm{d} \Omega}=\sigma^{R} \frac{1}{4 \pi^{2}\left(m_{\mathrm{e}} c\right)^{2}} \frac{e^{2}}{\hbar c} \frac{1}{\hbar \omega}\left(\boldsymbol{q} \times \hat{e}_{\omega}\right)^{2},
$$

where $\hat{e}_{\omega}$ is the unit vector in the direction of the emitted photon. By combining Equations (2.3.35) and (2.3.36), the AB cross section can be expressed by (Ishii et al., 2008)

$$
\frac{\mathrm{d}^{2} \sigma^{\mathrm{AB}+\mathrm{RI}}}{\mathrm{d}(\hbar \omega) \mathrm{d} \Omega}=\int_{0}^{\infty} \mathrm{d} v f_{i}(v) \int_{\hbar \omega}^{\infty} \mathrm{d} E \int \mathrm{d} q \sigma^{R} \frac{1}{4 \pi^{2}\left(m_{\mathrm{e}} c\right)^{2}} \frac{e^{2}}{\hbar c} \frac{1}{\hbar \omega}\left(\boldsymbol{q} \times \hat{e}_{\omega}\right)^{2} .
$$

Since the energy transfer is integrated from $\hbar \omega$ to $\infty$, both $\mathrm{AB}$ and RI processes are taken into account by this equation. Hence, in this approach, the $\mathrm{AB}$ calculations implicitly include the RI 
process.

Equation (2.3.37) can be integrated analytically over $\mathrm{d} E$ and $\mathrm{d} q$, and the result expressed as (Ishii et al., 2008)

$$
\frac{\mathrm{d}^{2} \sigma^{\mathrm{AB}+\mathrm{RI}}}{\mathrm{d}(\hbar \omega) \mathrm{d} \Omega}=C_{1 i}-C_{2 i}+\frac{1}{2}\left(3 C_{2 i}-C_{1 i}\right) \sin ^{2} \theta
$$

where the terms $C_{1 i}$ and $C_{2 i}$ are given by

$$
\begin{aligned}
C_{1 i}=\frac{1}{\pi\left(m_{\mathrm{e}} c\right)^{2}} & \frac{e^{6}}{\hbar c} \frac{1}{\hbar \omega} \frac{Z_{p}^{2}}{v_{\mathrm{p}}^{2}} \sum_{i} N_{i}\left\{\int _ { 0 } ^ { v _ { 1 } } \mathrm { d } v f _ { i } ( v ) \left[\ln \left(\frac{2 m_{\mathrm{e}} v_{\mathrm{p}}\left(v_{\mathrm{p}}-v\right)}{\hbar \omega}\right) \times\right.\right. \\
\times & \left.\frac{v_{\mathrm{p}}}{v}\left(1+\frac{v}{2 v_{\mathrm{p}}}\right) \ln \left(\frac{v_{\mathrm{p}}+v}{v_{\mathrm{p}}-v}\right)-\frac{1}{2}\left(4-\ln \left(1-\frac{v^{2}}{v_{\mathrm{p}}^{2}}\right)\right)\right]+ \\
& +\int_{v_{2}}^{\infty} \mathrm{d} v f_{i}(v)\left[\frac{1}{2}\left(1+2 \frac{v_{\mathrm{p}}}{v}\right) \ln \left(\frac{2 m_{\mathrm{e}} v_{\mathrm{p}}\left(v_{\mathrm{p}}+v\right)}{\hbar \omega}\right)-\right. \\
-(1+ & \left.\left.\left.\left.2 \frac{v_{\mathrm{p}}}{v}\right)+\sqrt{1+\frac{2 \hbar \omega}{m_{\mathrm{e}} v^{2}}-\frac{1}{2} \ln \left(\frac{2 \hbar \omega}{m_{\mathrm{e}} v^{2}}+1\right.}\right)\right]\right)
\end{aligned}
$$




$$
\begin{aligned}
& C_{2 i}=\frac{1}{\pi\left(m_{\mathrm{e}} c\right)^{2}} \frac{e^{6}}{\hbar c} \frac{1}{\hbar \omega} \frac{Z_{p}^{2}}{v_{\mathrm{p}}^{2}} \sum_{i} N_{i}\left\{\int _ { 0 } ^ { v _ { 1 } } \mathrm { d } v f _ { i } ( v ) \left[1-\frac{v}{v_{\mathrm{p}}}-\frac{\hbar \omega}{2 m_{\mathrm{e}} v_{\mathrm{p}}^{2}}+\right.\right. \\
& +\frac{1}{3}\left(\frac{v}{v_{\mathrm{p}}}\right)^{2} \ln \left(\frac{2 m_{\mathrm{e}} v_{\mathrm{p}}\left(v_{\mathrm{p}}-v\right)}{\hbar \omega}\right)+\frac{1}{3} \frac{v_{\mathrm{p}}}{v}\left(1+\frac{1}{2}\left(\frac{v}{v_{\mathrm{p}}}\right)^{3}\right) \ln \left(\frac{v_{\mathrm{p}}+v}{v_{\mathrm{p}}-v}\right)+ \\
& \left.+\frac{v_{\mathrm{p}}}{v}-\frac{1}{18}\left(12+\left(\frac{v}{v_{\mathrm{p}}}\right)^{2}\right)-\frac{1}{6}\left(\frac{v}{v_{\mathrm{p}}}\right)^{2}\left(4 \frac{v}{v_{\mathrm{p}}}-\ln \left(1-\left(\frac{v}{v_{\mathrm{p}}}\right)^{2}\right)\right)\right]+ \\
& +\int_{v_{2}}^{\infty} \mathrm{d} v f_{i}(v)\left[\frac{1}{3} \frac{v_{\mathrm{p}}}{v}\left(1+\frac{1}{2}\left(\frac{v}{v_{\mathrm{p}}}\right)^{3}\right) \ln \left(\frac{2 m_{\mathrm{e}} v_{\mathrm{p}}\left(v_{\mathrm{p}}+v\right)}{\hbar \omega}\right)+\right. \\
& +\frac{1}{2}\left(\left(1+\frac{v}{v_{\mathrm{p}}}\right)-\frac{\hbar \omega}{2 m_{\mathrm{e}} v_{\mathrm{p}}^{2}}\right)-\frac{v_{\mathrm{p}}}{36 v}\left(\left(\frac{v}{v_{\mathrm{p}}}+2\right)^{3}-\left(\sqrt{\left(\frac{v}{v_{\mathrm{p}}}\right)^{2}+\frac{2 \hbar \omega}{m_{\mathrm{e}} v_{\mathrm{p}}^{2}}}\right)^{3}\right)+ \\
& -\frac{v}{3 v_{\mathrm{p}}}\left(\frac{v}{v_{\mathrm{p}}}+2-\sqrt{\left(\frac{v}{v_{\mathrm{p}}}\right)^{2}+\frac{2 \hbar \omega}{m_{\mathrm{e}} v_{\mathrm{p}}^{2}}}\right)+ \\
& \left.\left.-\frac{1}{6}\left(\frac{v}{v_{\mathrm{p}}}\right)^{2} \ln \left(\frac{\sqrt{1+\frac{2 \hbar \omega}{m_{\mathrm{e}} v^{2}}}+1}{\left(1+\frac{v}{v_{\mathrm{p}}}\right)\left(\sqrt{1+\frac{2 \hbar \omega}{m_{\mathrm{e}} v^{2}}}-1\right)}\right)\right]\right\}
\end{aligned}
$$

Finally, the limits of the integrals over $\mathrm{d} v$ in the above expressions for the terms $C_{1 i}$ and $C_{2 i}$ are given by

$$
\left\{\begin{array}{ll}
v_{1}=v_{2}=v_{\mathrm{p}}\left(1-\frac{\hbar \omega}{2 m_{\mathrm{e}} v_{\mathrm{p}}^{2}}\right) & \text { for } \hbar \omega<2 m_{\mathrm{e}} v_{\mathrm{p}}^{2} \\
v_{1}=0, v_{2}=v_{\mathrm{p}}\left(\frac{\hbar \omega}{2 m_{\mathrm{e}} v_{\mathrm{p}}^{2}}-1\right) & \text { for } \hbar \omega>2 m_{\mathrm{e}} v_{\mathrm{p}}^{2}
\end{array} .\right.
$$

In the above expressions, $N_{i}$ and $f_{i}(v)$ are the number of electrons in the $i$ shell and their VD, respectively. Ishii et al. (2008) assumed the VD of the electrons to be given by the $1 \mathrm{~s}$ hydrogen wave function (see Equation (2.3.15)) for all shells of the target atom.

Figure 2.9 shows the comparisons between experiment and Equation (2.3.38) for silver and gold targets, a beam of protons with a kinetic energy of $1.5 \mathrm{MeV}$ and a photon emission angle of $\theta=90^{\circ}$. When comparing these results with Figure 2.8, obtained using the PWBA theory, it 
(a) Ag target

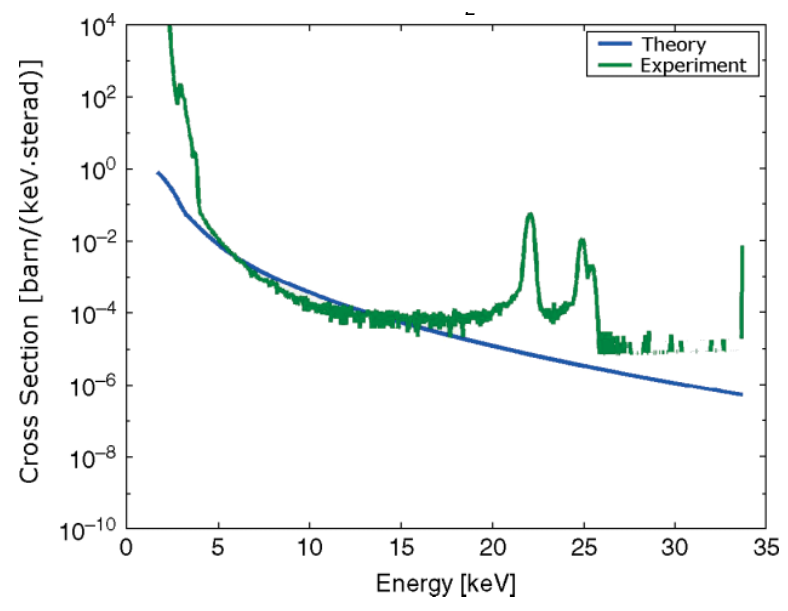

(b) Au target

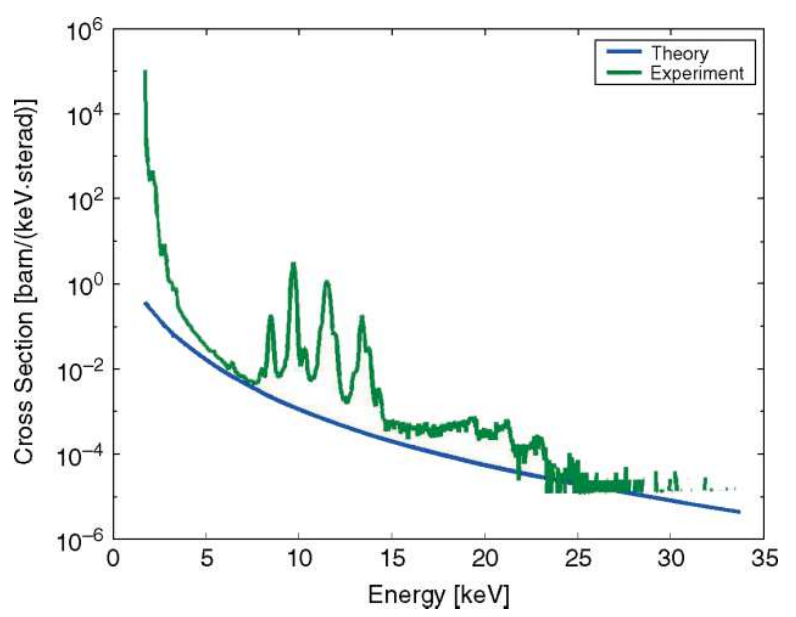

Figura 2.9: $A B$ cross sections of (a) silver and (b) gold targets bombarded with $1.5 \mathrm{MeV}$ protons and measured at $90^{\circ}$. The solid line shows the calculation within the BEA approximation. Source: Ishii et al. (2008).

can be noted that the $\mathrm{BEA}$ calculations of the $\mathrm{AB}$ process do not overestimate the results as much as the PWBA approach. Therefore, Ishii et al. (2008) concluded that Equations (2.3.38)-(2.3.40), derived in the $\mathrm{BEA}$, must be used to calculate the $\mathrm{AB}$ process.

\subsubsection{Quasi-Free Electron Bremsstrahlung}

In the Quasi-Free Electron Bremsstrahlung (QFEB) process, the target orbital electrons are seen as incident particles in the projectile's reference frame. If they are scattered by the Coulomb field of the proton, they can produce radiation, as illustrated in Figure 2.10.

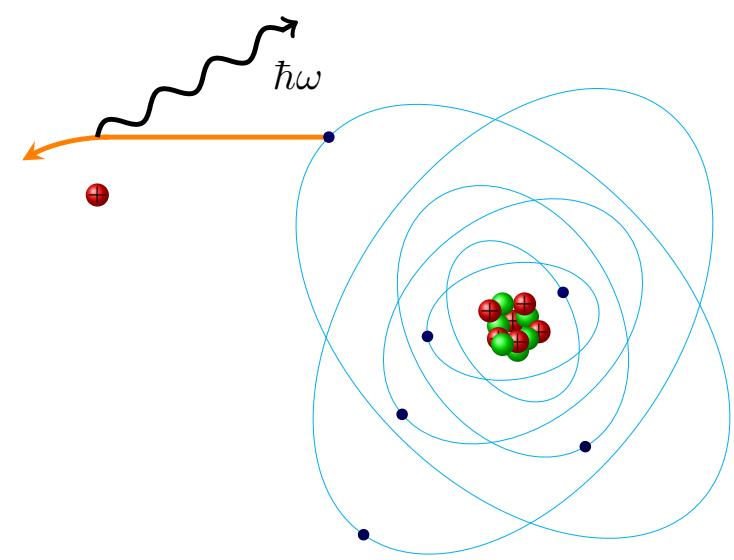

Figura 2.10: Schematic representation of the quasi-free electron bremsstrahlung (QFEB) process. 
As a first step, the electron is described as being free and at rest, so that its orbital speed, $v_{e}$, is not taken into account. Note that such a condition requires proton energies much above those of interest here. In this case, the kinetic energy of the orbital electron in the reference frame of the proton is given by Equation (2.3.9b). The cross section for the electronbremsstrahlung production is calculated in the PWBA theory for the relativistic case, without screening effect and considering the Sommerfeld correction. It is thus expressed, in the proton reference system, by (Chu et al., 1981; Heitler, 1954)

$$
\frac{\mathrm{d}^{2} \sigma^{\mathrm{QFEB}}}{\mathrm{d}(\hbar \omega) \mathrm{d} \Omega}=\left\{\begin{array}{l}
\frac{Z_{p}^{2} Z}{\pi}\left(\frac{e^{2}}{m_{\mathrm{e}} c^{2}}\right)^{2} \alpha \frac{m_{\mathrm{e}} c^{2}}{\hbar \omega T_{\mathrm{QF}}} g\left(\xi_{0}, \xi\right)\left\{\left[\sin ^{2} \theta+\frac{1}{4}(1+\mathcal{T})\left(3 \cos ^{2} \theta-1\right)\right]\right. \\
\quad \times \ln \left(\frac{1+\mathcal{T}}{1-\mathcal{T}}\right)+\frac{\beta_{\mathrm{QF}}}{2} \cos \theta\left[(7-\mathcal{T}) \sin ^{2} \theta+\frac{1}{2}\left(\frac{5}{2} \cos ^{2} \theta-\frac{3}{2}\right)\right. \\
\left.\times\left(10 \mathcal{T}+3-3 \mathcal{T}^{2}\right)\right]-\frac{1}{2} \sqrt{\mathcal{T}}\left(3 \cos ^{2} \theta-1\right) \\
\left.-2 \beta_{\mathrm{QF}} \sqrt{\mathcal{T}} \cos \theta\left(3 \cos ^{2} \theta-2\right)\right\}, \quad \text { for } \hbar \omega \leq T_{\mathrm{QF}} \\
0, \quad \text { for } \hbar \omega \geq T_{\mathrm{QF}}
\end{array}\right.
$$

In the above equation, $T_{\mathrm{QF}}$ and $\beta_{\mathrm{QF}}=\left(2 T_{\mathrm{QF}} / m_{\mathrm{e}} c^{2}\right)^{1 / 2}$ are the energy and the speed of the radiating electron, respectively, $\mathcal{T}=\left(T_{\mathrm{r}}-\hbar \omega\right) / T_{\mathrm{r}}$, the angle $\theta$ of radiation emission is relative to the direction of the incident electron, and, finally, $g\left(\xi_{0}, \xi\right)$ is the Sommerfeld correction term (see Section 2.2 and Equation (2.2.4)), with

$$
\begin{aligned}
& \xi_{0}=Z_{p} \sqrt{\mathcal{R} / T_{\mathrm{QF}}}, \\
& \xi=Z_{p} \sqrt{\mathcal{R} /\left(T_{\mathrm{QF}}-\hbar \omega\right)} .
\end{aligned}
$$

Equations (2.3.43) are equivalent to the definitions given after Equation (2.2.4) with $\beta=\beta_{\mathrm{QF}}$ and $\mathcal{R}=m_{\mathrm{e}} c^{2} \alpha^{2} / 2$, the Rydberg constant.

Nonetheless, in order to compare the theory with experimental data, it is necessary to express Equation (2.3.42) in the laboratory frame. This is accomplished by a Lorentz transformation (Chu et al., 1981; Jackson, 1999)

$$
\begin{aligned}
& \hbar \omega=\frac{1-\beta_{\mathrm{QF}} \cos \theta_{L}}{1-\beta_{\mathrm{QF}}^{2}} \hbar \omega_{L}, \\
& \cos \theta=\frac{\cos \theta_{L}-\beta_{\mathrm{QF}}}{1-\beta_{\mathrm{QF}} \cos \theta_{L}}
\end{aligned}
$$

where the subscript $L$ indicates that the variables are measured in the laboratory reference 
frame. The cross section is thus obtained by

$$
\frac{\mathrm{d}^{2} \sigma^{\mathrm{QFEB}}}{\mathrm{d}\left(\hbar \omega_{L}\right) \mathrm{d} \Omega_{L}}\left(T_{\mathrm{QF}}, \hbar \omega_{L}, \theta_{L}\right)=\frac{1-\beta^{2}}{1-\beta \cos \theta_{L}} \frac{\mathrm{d}^{2} \sigma^{\mathrm{QFEB}}}{\mathrm{d}(\hbar \omega) \mathrm{d} \Omega}\left(T_{\mathrm{QF}}, \hbar \omega, \theta\right)
$$

In the present first step, where the electron is initially at rest in the reference of the target atom, $\beta_{\mathrm{QF}}=v_{\mathrm{p}} / c$ and $T_{\mathrm{QF}}=T_{\mathrm{r}}$ where $T_{\mathrm{r}}$ is given by Equation (2.3.9b).

As a second step, the approximation of an electron being free and at rest can be further refined by taking into account an electron with velocity components $\left(v_{x}, v_{y}, v_{z}\right)$. This improved description can be used for proton energies that are lower than those necessary for the previous description, with an electron initially at rest, to be accurate. Considering that the $z$ axis is taken in the direction of the incident beam, the projectile has velocity components $\left(0,0, v_{\mathrm{p}}\right)$. In this case, the relative kinetic energy of the electron is given by

$$
\begin{aligned}
T_{\mathrm{r}}^{\prime} & =\frac{1}{2} m_{\mathrm{e}}\left[v_{x}^{2}+v_{y}^{2}+\left(v_{\mathrm{p}}-v_{z}\right)^{2}\right] \\
& =T_{\mathrm{r}}\left[\left(\frac{v_{x}}{v_{\mathrm{p}}}\right)^{2}+\left(\frac{v_{y}}{v_{\mathrm{p}}}\right)^{2}+\left(1-\frac{v_{z}}{v_{\mathrm{p}}}\right)^{2}\right] \\
& \approx T_{\mathrm{r}}\left(1-\frac{v_{z}}{v_{\mathrm{p}}}\right)^{2} .
\end{aligned}
$$

The approximation in Equation (2.3.46) is valid for $v_{\mathrm{p}} \gg v_{e}$, so the $z$ component is the only one that contributes to $T_{\mathrm{r}}^{\prime}$.

Now, the PDFs for the velocity component in the $z$ direction for the orbital electrons must be considered. This is obtained by

$$
f_{z i}\left(v_{z}\right)=\sum_{i} N_{i} \iint f_{i}\left(v_{x}, v_{y}, v_{z}\right) \mathrm{d} v_{x} \mathrm{~d} v_{y},
$$

where $f_{i}\left(v_{x}, v_{y}, v_{z}\right)$ is the VD for the $N_{i}$ electrons in the $i$-th atomic shell. The summation indicates that the contribution of all shells are added. If, following Chu et al. (1981), the hydrogen atom wave functions are employed, the $\operatorname{VD} f_{i}$ can be calcuated analytically and the PDF for the velocity component in the $z$ direction $f_{z i}$ as well. For example, orbitals $1 \mathrm{~s}$ and $2 \mathrm{~s}$ 
Tabela 2.2: Proton energies and corresponding electron kinetic energies used in the calculation of the QFEB process.

\begin{tabular}{|c|c|}
\hline$E_{\mathrm{p}}(\mathrm{MeV})$ & $T_{\mathrm{r}}(\mathrm{keV})$ \\
\hline 1 & 0.545 \\
\hline 4 & 2.179 \\
\hline 20 & 10.892 \\
\hline
\end{tabular}

give

$$
\begin{array}{ll}
f_{1 s}(v)=\frac{32 v_{0}^{5}}{\pi} \frac{v^{2}}{\left(v_{0}^{2}+v^{2}\right)^{4}} & \Rightarrow f_{z 1 s}\left(v_{z}\right)=\frac{8}{3 \pi} \frac{v_{0}^{5}}{\left(v_{0}^{2}+v_{z}^{2}\right)^{3}} \\
f_{2 s}(v)=\frac{256 v_{0}^{5}}{\pi^{2}} \frac{\left(4 v^{2}-v_{0}^{2}\right)^{2}}{\left(4 v^{2}+v_{0}^{2}\right)^{6}} \quad \Rightarrow \quad f_{z 2 s}\left(v_{z}\right)=\frac{128 v_{0}^{5}}{15 \pi} \frac{\left(40 v_{z}^{4}-10 v_{0}^{2} v_{z}^{2}+v_{0}^{4}\right)}{\left(4 v_{z}^{2}+v_{0}^{2}\right)^{5}}
\end{array}
$$

where, as usual, $U_{i}=\frac{1}{2} m_{\mathrm{e}} v_{i}^{2}$ is the binding energy of the respective shell.

Finally, the cross section for the QFEB process is obtained using Equations (2.3.45), (2.3.46) and (2.3.47) and integrating over the velocities in the $z$ direction (Chu et al., 1981)

$$
\frac{\mathrm{d}^{2} \sigma^{\mathrm{QFEB}}}{\mathrm{d}\left(\hbar \omega_{L}\right) \mathrm{d} \Omega_{L}}\left(\hbar \omega_{L}, \theta_{L}\right)=\int_{-\infty}^{v_{\mathrm{p}}\left(1-\left(\hbar \omega / T_{\mathrm{r}}\right)^{1 / 2}\right)} \frac{\mathrm{d}^{2} \sigma^{\mathrm{QFEB}}}{\mathrm{d}\left(\hbar \omega_{L}\right) \mathrm{d} \Omega_{L}}\left(T_{\mathrm{r}}^{\prime}, \hbar \omega_{L}, \theta_{L}\right) f_{z}\left(v_{z}\right) \mathrm{d} v_{z}
$$

where now $T_{\mathrm{QF}}=T_{\mathrm{r}}^{\prime}$ and, as above, $\beta_{\mathrm{QF}}=\left(2 T_{\mathrm{QF}} / m_{\mathrm{e}} c^{2}\right)^{1 / 2}$.

The upper limit in the the integration of Equation (2.3.49) is determined by the fact that the maximum photon energy is the relative kinetic energy of the electron, that is

$$
T_{\mathrm{r}}^{\prime}=T_{\mathrm{r}}\left(1-\frac{v_{z}}{v_{\mathrm{p}}}\right)^{2} \geq \hbar \omega \Rightarrow v_{z}^{\max }=v_{\mathrm{p}}\left[1-\left(\frac{\hbar \omega}{T_{\mathrm{r}}}\right)^{1 / 2}\right]
$$

Therefore, the QFEB cross section is characterized by the relative kinetic energy $T_{\mathrm{r}}$. This quantity can be written as $T_{\mathrm{r}}=\left(m_{\mathrm{e}} / m_{\mathrm{p}}\right) E_{\mathrm{p}}$, where $E_{\mathrm{p}}$ is the proton kinetic energy. So the maximum photon energy is reduced by a factor $\left(m_{\mathrm{e}} / m_{\mathrm{p}}\right)$ relative to the proton energy. The QFEB spectrum is thus limited to very low energies when compared to the other processes. Table 2.2 shows examples of the relative kinetic energies for proton energies of 1,4 and $20 \mathrm{MeV}$.

To validate Equations (2.3.45) and (2.3.49), Chu et al. (1981) compared to experimental data for $19.22 \mathrm{MeV}$ protons impinging on a beryllium target and emitting photons at $\theta_{L}=90^{\circ}$. The energy of $19.22 \mathrm{MeV}$ was estimated by Chu et al. (1981) to take into account the energy 


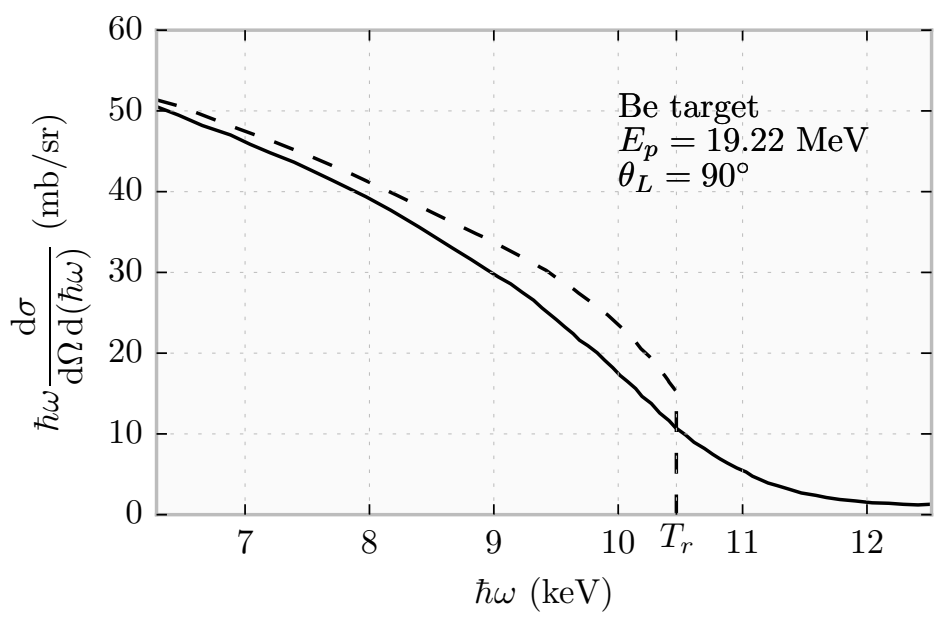

Figura 2.11: QFEB spectrum near the end-point energy, for a beryllium target and $19.22 \mathrm{MeV}$ protons, calculated by Chu et al. (1981). The solid line takes into account the VD of the orbital electrons, in contrast to the dashed line, which does not include this effect.

lost by the incident beam when penetrating the target. Besides, they subtract the contribution of secondary electron bremsstrahlung (for definition, see Section 2.3.1) by approximating it with a function of the form $\sum_{n=0} a_{n}\left(\theta_{L}\right)(\ln \hbar \omega)^{n}$, where the coefficients $a_{n}$ are determined from least-squares fit to the experimental spectrum. As can be seen in their paper (Chu et al., 1981), the authors obtain good agreement with Equation (2.3.49).

Figure 2.11 shows only the spectra near the end-point energy, calculated by Chu et al. (1981), in order to see the effects of the VD of the electron on the QFEB spectrum. The dashed curve shows the results for Equation (2.3.45), without the VD of the orbital electrons. On the other hand, the solid curve, obtained from Equation (2.3.49), considers the VD from 1s and 2s electrons (Equation (2.3.48)). In the first case, the spectrum presents a sharp high-energy cut-off, at $T_{\mathrm{r}}=10.468 \mathrm{keV}$, as expected. In the other case, however, the spectrum continues beyond $T_{\mathrm{r}}$ and gradually decrease to zero. This results clearly show the effect of the VD of the orbital electrons on the QFEB process.

\subsubsection{Nuclear Bremsstrahlung}

The Nuclear Bremsstrahlung (NB) process refers to the radiation produced by the projectile through Coulomb scattering by the atomic nucleus in the target material, as illustrated in Figure 2.12. In this case, the bremsstrahlung production cross section is calculated by first Born approximation and can be expressed by the following equation (Heitler, 1954; Ishii, 2006; Jackson, 1999): 


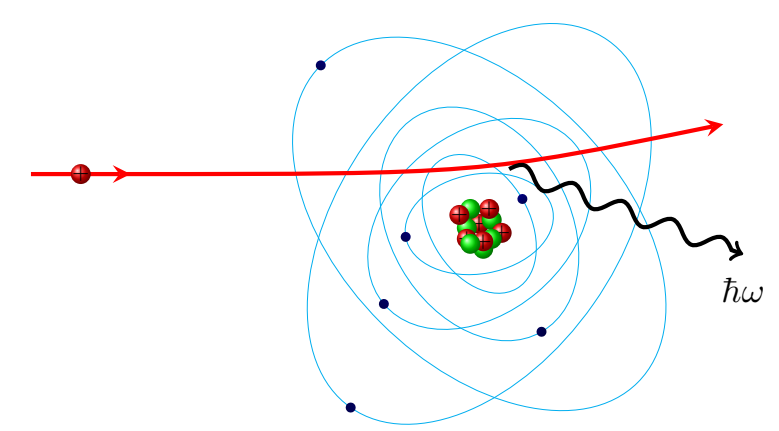

Figura 2.12: Schematic representation of the nuclear bremsstrahlung (NB) process.

$$
\begin{aligned}
\frac{\mathrm{d}^{2} \sigma^{\mathrm{NB}}}{\mathrm{d}(\hbar \omega) \mathrm{d} \Omega}= & \frac{Z_{\mathrm{p}}^{4} Z^{2}}{\pi \hbar \omega}\left(\frac{e^{2}}{m_{\mathrm{e}} c^{2}}\right)^{2}\left(\frac{m_{\mathrm{e}}}{m_{\mathrm{p}}}\right)^{2} \frac{e^{2}}{\hbar c}\left(\frac{c}{v_{\mathrm{p}}}\right)^{2}\left(1-\frac{m_{\mathrm{p}} Z}{Z_{\mathrm{p}} M}\right)^{2} \times \\
& \times\left[\ln \frac{4 E_{\mathrm{p}}}{\hbar \omega}-\ln \frac{Z_{\mathrm{p}} Z e^{2}}{\hbar v_{\mathrm{p}}}-\frac{1}{2}-\frac{1}{2}\left(\ln \frac{4 E_{\mathrm{p}}}{\hbar \omega}-\ln \frac{Z_{\mathrm{p}} Z e^{2}}{\hbar v_{\mathrm{p}}}-\frac{3}{2}\right) \sin ^{2} \theta\right],
\end{aligned}
$$

where $M$ is the atomic mass of the target material.

Equation (2.3.50) represents the doubly differential cross section (DDCS) for the NB process as a function of the photon energy. Figure 2.13 shows the results calculated by Ishii et al. (1999) for aluminum target bombarded with $1.5 \mathrm{MeV}$ protons. The graph compares values for $\theta=45^{\circ}$ and $\theta=90^{\circ}$, where $\theta$ is the photon emission angle. In this case, it is clear that the bremsstrahlung cross section is smaller at $\theta=90^{\circ}$.

As it will be seen, the cross section for NB is much smaller than the AB or the SEB ones in the photon energy region of interest for the present work. In fact, when compared to electron-nucleus bremsstrahlung, the NB cross section is reduced by a factor $\left(m_{\mathrm{e}} / m_{\mathrm{p}}\right)^{2}$, because the Coulomb forces are the same in electron-nucleus and proton-nucleus scattering, but the masses differ by a factor of $m_{\mathrm{p}} / m_{\mathrm{e}}=1836$. So the proton experiences much less deceleration and thus the intensity of its emitted radiation is much smaller than that of the electrons.

The factor $\ln \left(Z_{\mathrm{p}} Z e^{2} / \hbar v_{\mathrm{p}}\right)$ in Equation (2.3.50) was introduced by Alder et al. (1956) to account for target nuclei with higher atomic numbers. In the case of light-atom target and $\mathrm{MeV}$ incident protons, this term can be neglected in comparison to the factor $\ln \left(4 E_{\mathrm{p}} /(\hbar \omega)\right)$.

It can also be noted that, depending on the projectile and target element, the term $\left(1-\left(m_{\mathrm{p}} Z / Z_{\mathrm{p}} M\right)\right)^{2}$ might cancel the nuclear bremsstrahlung emission. In this case, the bremsstrahlung is dominated by the electric quadrupole emission Jakubaßa-Amundsen and Kleber 


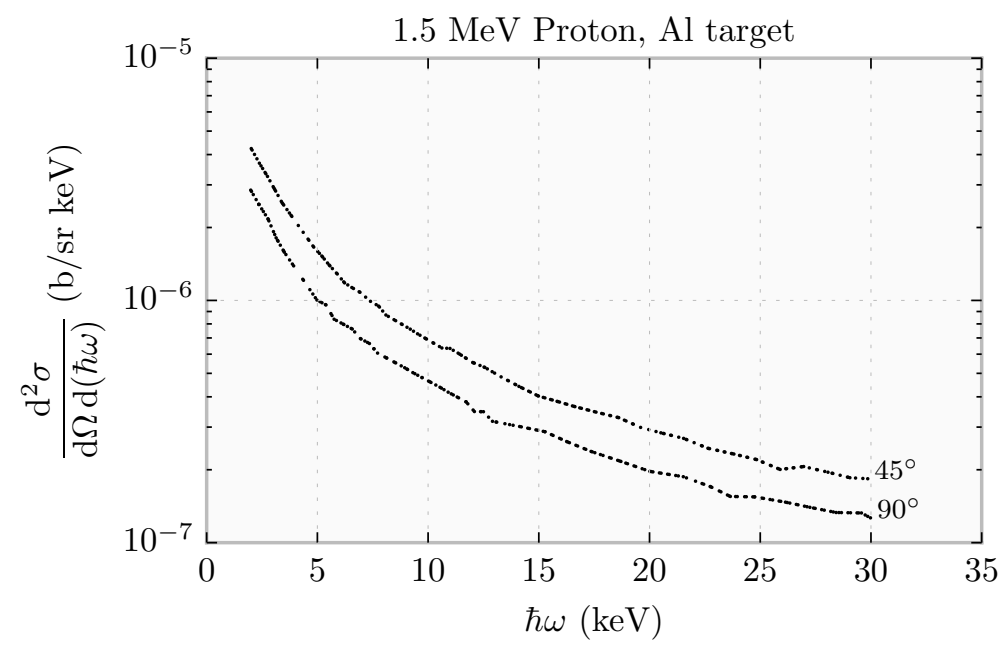

Figura 2.13: Nuclear bremsstrahlung cross sections for aluminum target and $1.5 \mathrm{MeV}$ protons Ishii et al. (1999).

(1975), whereas Equation (2.3.50) accounts for the electric dipole only (Alder et al., 1956). 


\subsection{PIXE}

In the PIXE analytical method, charged particles are produced in an accelerator and enter a vacuum chamber with a prepared sample of the material to be analyzed. These particles induce ionization of the atoms in the specimen, causing the electrons in the shells to rearrange and, therefore, emit characteristic x-rays or Auger electrons. However, x-rays are much easier to measure for practical reasons. The x-ray spectrum thus obtained is analyzed with suitable spectroscopic techniques in order to determine, in a non-destructive manner, the constituent elements of the material (Johansson et al., 1995).

Tandem accelerators used currently for PIXE are relatively small machines, generally operating with proton beams of energies between 1 and $4 \mathrm{MeV}$. The emitted x-rays are measured with semiconductor detectors, usually $\mathrm{Si}(\mathrm{Li})$ detectors, because of their suitable detection efficiency and energy resolution (see Chapter 4.2). PIXE can be applied to different types of specimens, most commonly being used to analyze thin films. In this case, the thickness of the specimen is such that incident particles lose small amounts of energy in its passage through the material, so that the energies at which the excitation of the target atoms occur are well defined. Moreover, the absorption of the emitted x-rays by the specimen is minimized (Johansson et al., 1995).

Figure 2.14 illustrates a typical PIXE spectrum obtained in archaeological investigations on the composition of nine small iron beads dated to circa 3200 BC, from Egypt (Rehren et al., 2013). This figure show that it is possible to infer the elements composing the material by identifying the characteristic peaks corresponding to the emission lines of each atomic species. Additionally, it can be seen that the continuous part of the spectrum affects the area of the peaks. This continuous spectrum is essentially composed of the bremsstrahlung from the impinging proton and the secondary electrons, besides the gamma ray production in nuclear reactions (Johansson et al., 1995).

A very important aspect of the IBA methods is the detection limit, i.e. the minimal element concentration that can be detected as a function of atomic number and projectile energy. In PIXE spectroscopy, one of the main factors determining the detection limit is the continuous spectrum superimposed on the characteristic x-ray peaks. Therefore, a quantitative approach to the PIXE method requires the knowledge of the cross sections for both the ionization and the bremsstrahlung processes.

There are no generally available programs yet to calculate the bremsstrahlung spectrum along with the characteristic x-rays, what imposes a limit to the PIXE technique in a selfconsistent approach. To work around this problem, usually a polynomial fit is performed to the background and extended to the area under the peaks, so the fitted background can be 


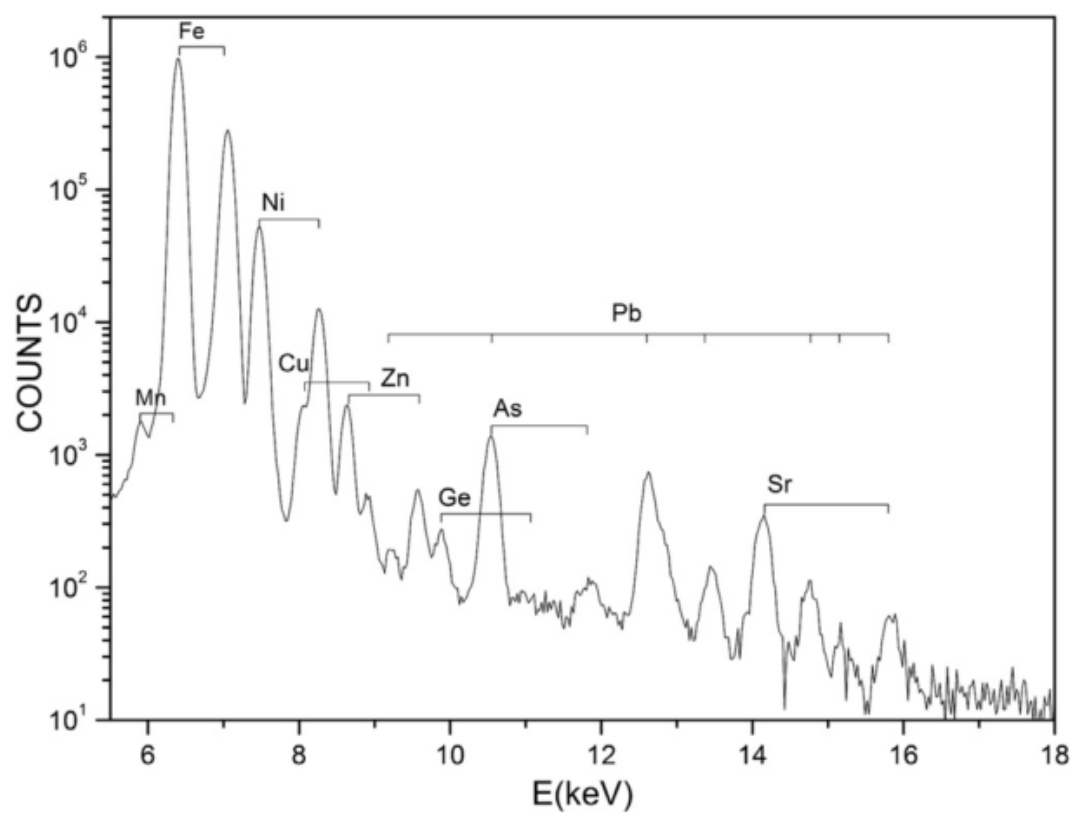

Figura 2.14: PIXE spectrum of an iron artifact dated to circa $3200 \mathrm{BC}$ showing the $\mathrm{K} \alpha$ and $\mathrm{K} \beta$ lines of the composing elements. Source: Rehren et al. (2013).

subtracted from the spectrum. As an example, in the case of Figure 2.14, the authors used the computer software GUPIX (Campbell et al., 2000) to process the data, which employs a top-hat digital filter to remove the background in order to calculate the area of the peaks (Rehren et al., 2013). This procedure, however, may lead to errors in the calculation of the area of the peaks, because the removed background is an approximation.

\subsubsection{Characteristic x-rays}

When an electron is ejected from the innermost shell of an atom by impact of a proton, another one from an outer shell of the atom fills that vacancy. The excess excitation energy results in the emission of $\mathrm{x}$-ray photons or auger electrons, in accordance with the selection rules of quantum mechanics and the energy levels of the elements in the specimen. Figure 2.15 illustrates a diagram for the transitions allowed in the emission of $\mathrm{x}$-rays by the usual selection rules $\Delta l= \pm 1$ and $\Delta j=0, \pm 1$.

The nomenclature of the transitions is given according to the initial and final electron shells. The K and L series, exemplified in the diagram of Figure 2.15, denote the transitions that occur towards K and L shells, respectively. Transitions occurring from L-shell to K-shell are called $\mathrm{K} \alpha$ lines; transitions occurring from $\mathrm{M}$-shell are called $\mathrm{K} \beta$ lines. Furthermore, due to fine structure splitting, both $\mathrm{K} \alpha$ and $\mathrm{K} \beta$ lines are doublets, as shown in the diagram. The $\mathrm{L}$ lines are more numerous for heavier elements, so that the total x-ray spectrum can be quite complex. 


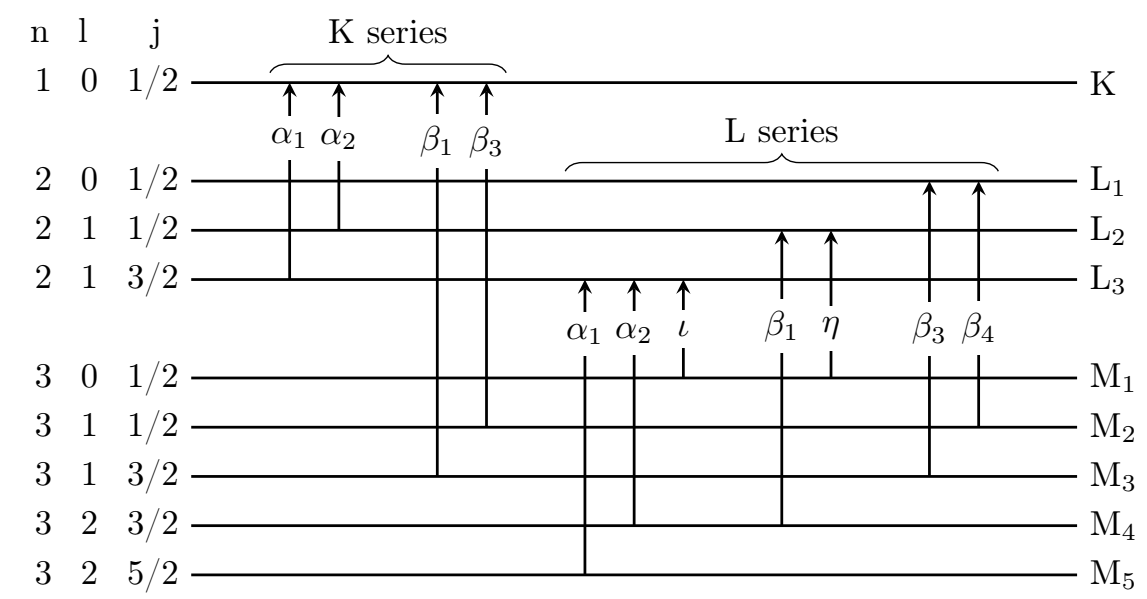

Figura 2.15: Diagram (not to scale) of $K$ and $L$ energy levels and transitions allowed by quantum mechanics selection rules $\Delta l= \pm 1$ and $\Delta j=0, \pm 1$.

The x-ray production cross sections of the $k$-th shells, $\sigma_{\mathrm{k}}^{\mathrm{X}}$, are determined by combining the following terms:

- Ionization cross sections, $\sigma_{\mathrm{k}}^{\mathrm{I}}$ (Pia et al., 2009);

- Coster-Kronig transition rates, $f_{\mathrm{k} i j}$ and $f_{\mathrm{k} i j}^{\prime}$, for a vacancy moving from the $i$-th subshell to the $j$-th subshell within the $k$ shell (Cohen et al., 2015; Schoonjans et al., 2011, 2015);

- Fluorescence yields, $\omega_{\mathrm{k}}$ for the transition to result in x-ray emission (Schoonjans et al., 2011, 2015);

- Line emission rates, $\Gamma_{\mathrm{k}}$ (Schoonjans et al., 2011, 2015).

Therefore, the x-ray production cross sections are given by (Cohen et al., 2015):

\section{K-shell:}

$$
\sigma_{\mathrm{K}}^{\mathrm{X}}=\sigma_{\mathrm{K}}^{\mathrm{I}} \omega_{\mathrm{K}} \Gamma_{\mathrm{K}}
$$

\section{L-shell:}

$$
\begin{aligned}
& \sigma_{\mathrm{L}_{1}}^{\mathrm{X}}=\sigma_{\mathrm{L}_{1}}^{\mathrm{I}} \omega_{\mathrm{L}_{1}} \Gamma_{\mathrm{L}_{1}} \\
& \sigma_{\mathrm{L}_{2}}^{\mathrm{X}}=\left(\sigma_{\mathrm{L}_{1}}^{\mathrm{I}} f_{\mathrm{L} 12}+\sigma_{\mathrm{L}_{2}}^{\mathrm{I}}\right) \omega_{\mathrm{L}_{2}} \Gamma_{\mathrm{L}_{2}} \\
& \sigma_{\mathrm{L}_{3}}^{\mathrm{X}}=\left(\sigma_{\mathrm{L}_{1}}^{\mathrm{I}}\left(f_{\mathrm{L} 12} f_{\mathrm{L} 23}+f_{\mathrm{L} 13}+f_{\mathrm{L}_{13}}^{\prime}\right)+\sigma_{\mathrm{L}_{2}}^{\mathrm{I}} f_{\mathrm{L} 23}+\sigma_{\mathrm{L}_{3}}^{\mathrm{I}}\right) \omega_{\mathrm{L}_{3}} \Gamma_{\mathrm{L}_{3}}
\end{aligned}
$$




\section{M-shell:}

$$
\begin{aligned}
\sigma_{\mathrm{M}_{1}}^{\mathrm{X}}= & \sigma_{\mathrm{M}_{1}}^{\mathrm{I}} \omega_{\mathrm{M}_{1}} \Gamma_{\mathrm{M}_{1}} \\
\sigma_{\mathrm{M}_{2}}^{\mathrm{X}}= & \left(\sigma_{\mathrm{M}_{1}}^{\mathrm{I}} f_{\mathrm{M} 12}+\sigma_{\mathrm{M}_{2}}^{\mathrm{I}}\right) \omega_{\mathrm{M}_{2}} \Gamma_{\mathrm{M}_{2}} \\
\sigma_{\mathrm{M}_{3}}^{\mathrm{X}}= & \left(\sigma_{\mathrm{M}_{1}}^{\mathrm{I}}\left(f_{\mathrm{M} 12} f_{\mathrm{M} 23}+f_{\mathrm{M} 13}\right)+\sigma_{\mathrm{M}_{2}}^{\mathrm{I}} f_{\mathrm{M} 23}+\sigma_{\mathrm{M}_{3}}^{\mathrm{I}}\right) \omega_{\mathrm{M}_{3}} \Gamma_{\mathrm{M}_{3}} \\
\sigma_{\mathrm{M}_{4}=}^{\mathrm{X}}= & \left(\sigma_{\mathrm{M}_{1}}^{\mathrm{I}}\left(f_{\mathrm{M} 12} f_{\mathrm{M} 23} f_{\mathrm{M} 34}+f_{\mathrm{M} 13} f_{\mathrm{M} 34}+f_{\mathrm{M} 12} f_{\mathrm{M} 24}+f_{\mathrm{M} 14}\right)+\right. \\
\sigma_{\mathrm{M}_{5}=}^{\mathrm{X}=} & \left(\sigma _ { \mathrm { M } _ { 1 } } ^ { \mathrm { I } } \left(f_{\mathrm{M} 12} f_{\mathrm{M} 23} f_{\mathrm{M} 34} f_{\mathrm{M} 45}+f_{\mathrm{M} 12} f_{\mathrm{M} 24} f_{\mathrm{M} 45}+f_{\mathrm{M} 12} f_{\mathrm{M} 23} f_{\mathrm{M} 35}+f_{\mathrm{M} 14} f_{\mathrm{M} 45}+f_{\mathrm{M} 13} f_{\mathrm{M} 35}+\right.\right. \\
& \left.\left.+f_{\mathrm{M} 12} f_{\mathrm{M} 25}+f_{\mathrm{M} 15}\right)+\sigma_{\mathrm{M}_{2}}^{\mathrm{I}}\left(f_{\mathrm{M} 23} f_{\mathrm{M} 34} f_{\mathrm{M} 45}+f_{\mathrm{M} 24} f_{\mathrm{M} 45}+f_{\mathrm{M} 25}\right)+\sigma_{\mathrm{M}_{4}}^{\mathrm{I}}\right) \omega_{\mathrm{M}_{4}} \Gamma_{\mathrm{M}_{4}} \\
& \left.+\sigma_{\mathrm{M}_{3}}^{\mathrm{I}}\left(f_{\mathrm{M} 34} f_{\mathrm{M} 45}+f_{\mathrm{M} 35}\right)+\sigma_{\mathrm{M}_{4}}^{\mathrm{I}} f_{\mathrm{M} 45}+\sigma_{\mathrm{M}_{5}}^{\mathrm{I}}\right) \omega_{\mathrm{M}_{5}} \Gamma_{\mathrm{M}_{5}}
\end{aligned}
$$

Although the cross sections for x-ray emission can be calculated using the equations given above, the experimental spectrum is heavily affected by inherent effects of the radiation detector. Due to the resolution of the detector, lines with close energy values, as for instance the doublet $\mathrm{K} \alpha_{1}$ and $\mathrm{K} \alpha_{2}$, can appear under the same peak, being thus indistinguishable. The efficiency of the detector is also an important aspect of the experiment, as it can suppress lines in some energy ranges. For example, the efficiency of $\mathrm{Si}(\mathrm{Li})$ detectors decreases rapidly for photon energies outside the range 5 to $30 \mathrm{keV}$, approximately (see Section 4.2.2).

Additionally, some transitions occur with low intensity and are often indistinguishable from the bremsstrahlung spectrum. Figure 2.16 shows the energy of characteristic x-ray lines as a function of atomic number, $Z$. As it can be seen, $\mathrm{K} x$-rays are efficiently detected, employing a $\mathrm{Si}(\mathrm{Li})$ sensor, for elements in the interval $20<Z<60$, whilst $\mathrm{L} x$-rays require $Z>60$. In practice, typical PIXE spectra for light-medium elements present good resolution for the $\mathrm{K} \alpha$ and $\mathrm{K} \beta$ peaks, though it is possible to observe L lines in case of heavier elements (Johansson et al., 1995).

\subsubsection{PIXE spectroscopy}

As an analytical method based on the x-ray emission from charged-particle-irradiated specimens, PIXE spectroscopy is intended to measure the energy distribution of the resulting $\mathrm{x}$ rays. In this case, the technique employs semiconductor diode detectors, typically of the lithium- 


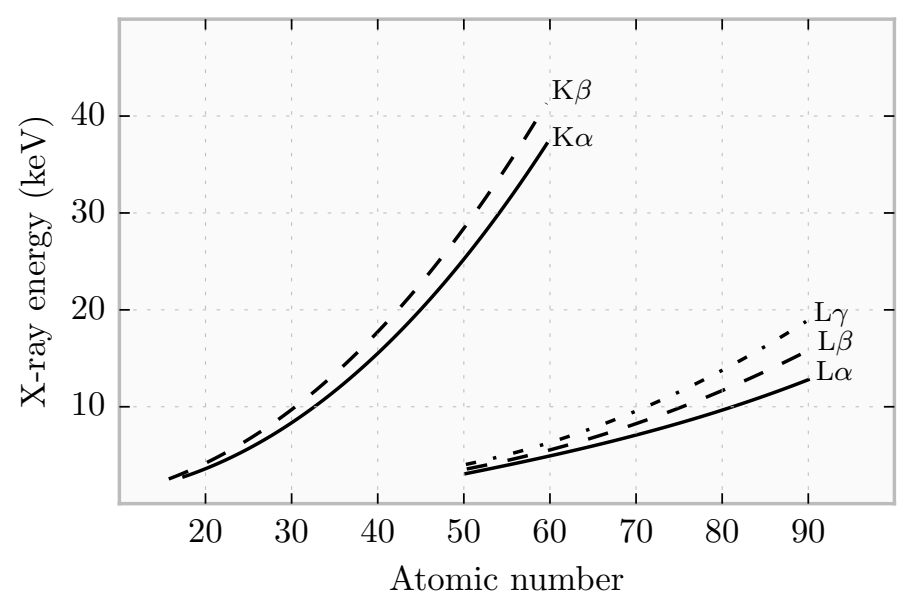

Figura 2.16: Energy of the main $\mathrm{K}$ and $\mathrm{L}$ transitions as a function of atomic number (Johansson et al., 1995).

drifted silicon type, $\mathrm{Si}(\mathrm{Li})$, as mentioned. When a charged particles impinges on a semiconductor detector, it losses energy by ionisation producing electron-hole pairs and, in analogy to the electron-ion pairs created in gas-filled detectors, an electric field is applied to generate the electrical signal. Therefore, these signals carry information about the charge generated by the radiation in the detection medium and are recorded in a pulse height spectrum. When the incident radiation is composed by neutral particles, like photons, it is first necessary that their energy is transferred to a charged particle, typically an atomic electron by a photoelectric or Compton interaction (see Subsection 2.4.3).

The analysis of the resulting PIXE spectra requires knowledge on the intrinsic characteristics of the detector, as well as some aspects of the electronics necessary for the data acquisition. Some properties of the detector have considerable influence on the resulting spectrum. In this sense, the use of semiconductors as the detection medium is of great importance in the PIXE technique. As opposed to a gas-filled detector, the solid detection medium allows the dimensions of the detector to be smaller, while maintaining relatively good detection efficiency and good energy resolution.

As will be shown in Chapter 4, the efficiency of a $\mathrm{Si}(\mathrm{Li})$ detector rapidly decreases for energies above $\sim 30 \mathrm{keV}$. In cases where the K-lines from elements of higher atomic number need to be recorded, high-purity germanium (HPGe) detectors are generally used to maintain detector efficiency up to $100 \mathrm{keV}$. However, because K-shell ionization cross sections from proton impact decrease with increasing atomic number, L-lines are more suitable for heavy target elements (see Figure 2.16), in which case $\mathrm{Si}(\mathrm{Li})$ detectors can be accordingly used.

Moreover, the energy resolution of the detector is reflected on the shape of the peak recorded with a source of monoenergetic radiation. Potential effects broadening the response 
peak are the noise of the electronics of the signal-processing system, deviations from the ideal operation of the detector, such as the leakage current fluctuations or charge collection non uniformities, and the statistical nature of the generation of charges in the detector. Thus the width of the response peak reflects the energy resolution of the detector. Higher energy resolutions mean smaller widths of the characteristic peaks, allowing to resolve closer emission lines. Typically, Si(Li) detectors present typical energy resolution values of about $150 \mathrm{eV}$ (FWHM), for $5.9 \mathrm{keV}$ photons (Johansson et al., 1995), which allows a good separation for most emission lines of neighboring elements.

In Chapter 4, some important aspects of detection effects in PIXE spectroscopy are discussed. These concepts are reviewed in order to be applied to theoretical calculations of the DDCSs for proton bremsstrahlung, thus enabling the comparison between the theories presented in Section 2.3 and experimental measurements performed at LAMFI, shown in Chapter 5. However, it is appropriate to briefly review the mechanisms of interaction of x-rays with matter, as presented in the following Section 2.4.3.

\subsubsection{Interactions of $\mathrm{x}$-rays with matter}

The processes of interaction of radiation with matter are crucial aspects of the operation of radiation detectors. In this sense, the x-ray photons incident on the detector must undergo some kind of interaction with the medium so that energy can be transferred to charged particles and a signal can be produced. In the case of radiation measurement in PIXE spectroscopy, which generally employs $\mathrm{Si}(\mathrm{Li})$ detectors, the most important interaction processes are the photoelectric absorption and the Compton scattering.

In these processes, the incident photon can be absorbed or deflected from its original path by a large angle while losing an amount of its energy. As a result, a beam a photons has its intensity attenuated when passing through the material. Besides, the cross sections for these processes are much smaller than that of the collisions of charged particles in matter. Therefore, $\mathrm{x}$-rays have a larger depth of penetration in the material when compared to charged particles.

\section{Photoelectric absorption}

The photoelectric absorption is an interaction of the photon with an atom as a whole, in which the photon is absorbed. The atom, in turn, ejects an energetic photoelectron, while the nucleus takes the recoil momentum. The photoelectric absorption by free electrons can not happen because of energy-momentum conservation. The ejected photoelectron has the energy given by

$$
E=\hbar \omega-U,
$$


where $U$ is the binding energy for the shell from which the electron has been ejected (the kinetic energy of the recoiling atom is very small and can be neglected).

Figure 2.17 shows the cross section for the photoelectric absorption (solid line) for silicon, as function of the photon energy. As it can be seen, the cross section has a discontinuity near the binding energy of the electrons in the K-shell. The sudden drop occurs because, below this point, the photon does not have enough energy to eject the photoelectron, which is bound in the atom. Such discontinuity is called the absorption edge, which, in this case, is the K absorption edge with an energy of $1.838 \mathrm{keV}$. As expected, further absorption edges, such as the $\mathrm{L}$ and $\mathrm{M}$ edges, are also observed in the photoelectric cross section.

In the case of a monoenergetic radiation source, the ideal process of photoelectric absorption, where no photons or photoelectrons escape from the detector, results in a single peak in the response function of the detector. This is called the full energy peak and it appears at the energy of the incident $\mathrm{x}$-ray photons.

In general, the resulting vacancy in the shell of the ionized atom can be filled by the rearrangement of the electrons from the other shells. Consequently, one or more characteristic $\mathrm{x}$-ray photons or Auger electrons can be emitted. In general, their energy is reabsorbed due to subsequent photoelectric effects or energy loss in the medium, respectively. However, there is the possibility of the escape of a characteristic x-ray from the detector, especially when it is produced near a surface of the sensitive volume. The escape of Auger electrons occurs with rather low probabilities, due to their small range in solid media. This phenomenon gives rise to the so called escape peak in the response function of the detector, as discussed in Section 4.2.1.

\section{Compton scattering}

The Compton scattering is an interaction between the $\mathrm{x}$-ray photon and an atomic electron. If the energy of the photon is much greater than the binding energy, the electron can be considered essentially free. In this process, the incident photon, with energy $\hbar \omega$, is scattered by the electron, which is initially at rest, through an angle $\theta$ relative to its original direction. The electron thus absorbs part of the energy of the photon and is scattered through an angle $\phi$ with respect to the direction of incidence of the photon.

The cross section for the Compton scattering process on a free electron is given by the Klein-Nishina formula (Knoll, 2000; Leo, 1994), which is a differential cross section for the angular distribution of the scattered photons. Its integration over the solid angle (Leo, 1994) gives the total cross section for the Compton scattering on a free electron.

In practice, however, the electrons are bound to the atom, so that the Klein-Nishina formula must be multiplied by a correction term called incoherent scattering function (Hubbell, 1969). The resulting cross section for the Compton scattering is illustrated in Figure 2.17 (dashed 
line) for the silicon atom. It can be seen that, for the energy range of interest to the present work (up to $\sim 20 \mathrm{keV}$ ), the cross section for the Compton scattering is much smaller than that of the photoelectric absorption.

The energy of the scattered photon, $\hbar \omega^{\prime}$, in terms of the scattering angle $\theta$, is given by (Knoll, 2000)

$$
\hbar \omega^{\prime}=\frac{\hbar \omega}{1+\left(\hbar \omega / m_{\mathrm{e}} c^{2}\right)(1-\cos \theta)},
$$

where $m_{\mathrm{e}} c^{2}=0.511 \mathrm{MeV}$ is the rest energy of the electron. An interesting limit case for this formula is that of a head-on collision $\theta=\pi$, in which the maximum transfer of energy from the photon to the recoil electron occurs. In this case, Equation (2.4.5) gives

$$
\left.\hbar \omega^{\prime}\right|_{\theta=\pi}=\frac{\hbar \omega}{1+2 \hbar \omega / m_{\mathrm{e}} c^{2}}
$$

Therefore, the maximum recoil energy, know as the Compton edge, is given by

$$
T_{C o m p}=\hbar \omega-\left.\hbar \omega^{\prime}\right|_{\theta=\pi}=\hbar \omega\left(\frac{2 \hbar \omega / m_{\mathrm{e}} c^{2}}{1+2 \hbar \omega / m_{\mathrm{e}} c^{2}}\right) .
$$

Because all scattering angles can occur in the detector, a continuum of recoil energy is observed, with a sharp drop corresponding to the Compton edge. In the present work, an upper limit for the energy of the photons can be considered to be of the order of $\hbar \omega=20 \mathrm{keV}$, for which Equation (2.4.7) gives $T_{\text {Comp }}=1.452 \mathrm{keV}$. The Compton edge is thus easier to see in spectra produced by high energy photons. In addition to the fact that the probability of a Compton scattering to occur is much smaller than that of the photoelectric absorption, the energy interval covered by the Compton continuum is small. Therefore, Compton scattering is not considered in the model of the response function of the detector (see Section 4.2).

\footnotetext{
${ }^{3}$ Source: https://www.nist.gov/pml/xcom-photon-cross-sections-database. Accessed on May 2018.
} 
(a)



(b)



Figura 2.17: Photoelectric absorption (solid lines) and Compton scattering (dashed lines) cross sections for silicon (a) and gold (b) ${ }^{3}$.

\section{Summary}

The emission of radiation from accelerated charged particles is called bremsstrahlung. In particular, the scattering of charged particles by the screened Coulomb field of atomic nuclei is associated with the emission of a continuous photon spectrum.

General aspects of the proton bremsstrahlung mechanisms have been revisited in this Chapter, along with the concepts involved in the available theories and the expressions currently in use. Additionally to the production of bremsstrahlung during the scattering of the proton in the field of a target atom, the impact of the projectile ejects target electrons within the sample. As a consequence, the secondary electrons also produce bremsstrahlung. In general, five processes of proton bremsstrahlung production must be considered: Nuclear Bremsstrahlung (NB), Secondary Electron Bremsstrahlung (SEB), Atomic Bremsstrahlung (AB), Radiative Ionization (RI), and Quasi-Free Electron Bremsstrahlung (QFEB).

In the NB process, the radiation is produced by the scattering of the proton in the Coulomb field of the target nucleus. The QFEB, on the other hand, refers to the radiation produced by an orbital electron of the target atom when regarded as the impinging particle in the reference frame of the proton. The other processes, $A B$ and $S E B$, account for the bremsstrahlung produced by target electrons ejected by the impact of the proton. In the SEB mechanism, the ejected electron loses energy as it travels through the material, and produces 
bremsstrahlung in the field of another nucleus.

In currently published works, the derivation of the SEB DDCS is based on the Binary Encounter Approximation (BEA) for the ionization DDCS. In such an approach to the ionization by impact of the proton, the collision is treated in terms of nonrelativistic quantum mechanics. The target electron is assumed to be free and the nucleus establishes its initial momentum distribution. In this sense, the model for the VD of the electrons has an important impact in the calculation of the ionization DDCS. In all previously published results for processes of proton bremsstrahlung where electrons are involved, their VD was derived from the square modulus of the wave function of the $1 \mathrm{~s}$ orbital of the hydrogen atom in momentum space. In the present work, this analytical expression has been presented along with the possibility of the implementation of numerical calculations with Dirac-Hartree-Fock-Slater (DHFS) VDs, suggesting an improvement to the calculations of the ionization DDCSs in the BEA and, consequently, the SEB or AB+RI DDCSs.

Finally, the PIXE analytical method is discussed. The technique can provide experimental data for the study of proton bremsstrahlung processes, as the PIXE spectrum is composed of the characteristic $\mathrm{x}$-rays and the continuous spectrum due to bremsstrahlung. However, a comparison with the theoretical calculations of the proton bremsstrahlung DDCSs requires the understanding of the interactions of radiation with matter. The concepts introduced in this part of the text are needed for the description of the modeling of the detector response function, discussed in Chapter 4. 


\section{Capítulo 3}

\section{Theoretical calculations}

In this chapter, an alternative calculation of the SEB DDCS is proposed. This approach addresses the inconsistencies present in the reference articles, which might possibly have occurred due to misprint issues. Here, the SEB DDCS is expressed in terms of an exponential integral function. In this way, the dimensions of the integral to be computed numerically are reduced from 5D to 3D. Moreover, the terms entering the SEB DDCS are analyzed in more details, namely the ionization and electron bremsstrahlung DDCSs and the energy loss formula.

In order to evaluate the currently available expressions of the proton bremsstrahlung DDCS, a FORTRAN code has been developed. The expressions presented in the previous chapter have been properly implemented according to the corresponding references, except for the SEB expressions. Moreover, the implemented calculations are based on the BEA, which depends on the VD of the electrons. As reviewed in the previous chapter, the analytical formula of the hydrogenic 1s VD has been adopted in all previously published works. Therefore, the hydrogenic VD has been used throughout the validation of the code to allow a comparison to the DDCSs available in the reference articles. After validating the code, an approach is proposed for the calculation of the ionization, SEB, and AB+RI DDCSs employing the VDs obtained with the Dirac-Hartree-Fock-Slater method.

In Section 3.1, the expression of the SEB DDCS is studied and its formulation based on the exponential integral functions is presented. In Section 3.2, a brief description of the main aspects of the code is presented. Lastly, in Section 3.3, the results of the calculations of each process are presented in comparison to the reference values.

\subsection{Secondary Electron Bremsstrahlung}

As studied in the previous chapter, SEB is one of the main components in the bremsstrahlung spectrum induced by the impact of protons. The process is described in two stages, 
where, firstly, the impinging proton ejects an electron from a target atom, which consequently looses energy as it moves through the medium; then, the secondary electron produces bremsstrahlung by scattering in the field of another nucleus.

Therefore, the SEB DDCS is expressed in terms of a 5D integral, namely over the speed of the electron in the orbital of the target atom $(\mathrm{d} v)$, the energy $\left(\mathrm{d} E_{\mathrm{e}}\right)$ and the angles $\left(\mathrm{d} \theta_{\mathrm{e}}, \mathrm{d} \varphi_{\mathrm{e}}\right)$ of the ejected electron, and the energy of the electron after a certain fraction has been lost in the material $\left(\mathrm{d} E_{\mathrm{e}}^{\prime}\right)$. Equation (2.3.2) defines the general expression of the SEB DDCS (Ishii et al., 1976). Alternatively, Equation (2.3.4) states the same DDCS in terms of more explicit expressions (Yamadera et al., 1981), where only the integral over the VD needs to be performed numerically while the other four have been performed analytically under some approximations.

However, despite all efforts for accurately implementing the numerical calculation of the SEB DDCS based on Equation (2.3.4), according to Yamadera et al. (1981), the numerical results show persistent discrepancies when compared to the plots from the reference article. Moreover, results from this implementation are consistently lower than the values obtained by Ishii et al. (1976), as will be show in the following.

A possible hint to the cause of the problem is the existence of small differences between the expressions for the $g_{2}(s)$ function (Equation (2.3.8)) in the paper by Yamadera et al. (1981) and in the review article by Ishii (2006). These small discrepancies suggest typographical misprints that would lead to incompatible results, which is possible to occur considering the complexity and length of the expressions. Indeed, because of such minor discrepancies in the $g_{2}(s)$ function, only the expression published by Yamadera et al. (1981) was implemented herein, because the other expression results in a discontinuous function.

Therefore, an alternative approach to express the SEB DDCS based on Equation (2.3.2) is proposed in the following sections. Before describing this alternative expression, an overview of the terms entering into the SEB DDCS, as first calculated by Ishii et al. (1976), is presented. This step allows to understand the approximations that have been made so far and to compare the results with some more recent and accurate ones.

Specifically, from the expression for the SEB DDCS given by Equation (2.3.2) (Ishii et al., 1976), the terms that will be reviewed here are: $\mathrm{d}^{2} \sigma_{i}^{\text {ion }} / \mathrm{d} E_{\mathrm{e}} \mathrm{d} \Omega_{\mathrm{e}}$, the ionization DDCS for impact of a proton with a given subshell; $S\left(E_{\mathrm{e}}^{\prime}\right)$, the energy loss formula for the ejected electron; and $\mathrm{d}^{2} \sigma^{\mathrm{br}} / \mathrm{d}(\hbar \omega) \mathrm{d} \Omega_{\mathrm{br}}$, the electron bremsstrahlung DDCS (Equation (2.3.3)).

\subsubsection{Ionization DDCS}

As discussed in Section 2.3.1.1, the BEA approach for the ionization DDCS brings in a dependency on the VD of the electrons in each subshell of the target atom. The ionization DDCS is defined in terms of the DDCS for ejection of an electron with a certain initial velocity 
by the impact of the impinging proton, which within the BEA, depends on the VD of the electrons of the shell, as can be seen in Equation (2.3.11). Moreover, the integration limits $v^{\text {min }}$ and $v^{\text {max }}$ in the ionization DDCS are defined by applying the laws of momentum and energy conservation, as summarized by Bonsen and Vriens (1970). Consequently, these limits depend on $E_{\mathrm{e}}$ and $\theta_{\mathrm{e}}$, besides $E_{\mathrm{p}}$, therefore determining the sequence in which the integrations must be performed in Equation (2.3.2).

Usually, the hydrogenic 1s VD has been adopted for all orbitals (Ishii, 2006; Ishii et al., 1976; Yamadera et al., 1981) in the calculations of the SEB DDCS, as well as the AB+RI and the QFEB DDCSs, which also depend on the VD of the electron. In this case, the formula for the VD of the electrons is obtained from the 1s hydrogenic wave function, as expressed in Equation (2.3.15), where $v_{0}$ is related to the binding energy, $U$, of the corresponding atomic shell as $U=\frac{1}{2} m_{\mathrm{e}} v_{0}^{2}$. In this chapter, however, use of numerical calculations of the PDFs from the DHFS method is proposed, in order to investigate the impact of the VD of the electrons on the SEB DDCS.

In this sense, calculations of the ionization DDCS in the BEA using the analytical hydrogenic VD can be validated using data from the reference articles (Bonsen and Vriens, 1970; Ishii et al., 1976). Additionally, the results applying the numerical DHFS PDFs can be directly compared with the results using the hydrogenic PDF. Nonetheless, the ionization DDCS based on the DHFS PDFs should also be studied in comparison to current values of the ionization DDCS calculated from other theories, rather than the BEA.

However, because of its applications in PIXE technique, as well as other IBA methods, most tables provide the total cross section for the ionization by impact of a proton, instead of the DDCS. The cross section for ionization of inner shells is largely used in the calculation of characteristic x-ray emission. Consequently, multiple data sources are available for the ionization cross section, mostly for inner shells (Pia et al., 2009). Those tables include data calculated from the most relevant models for the ionization process, such as the PWBA and the ECPSSR. Therefore, it is possible to investigate the accuracy of the BEA with realistic DHFS VDs on the calculation of the ionization cross section.

In order to obtain the total ionization cross section, it is necessary to integrate the corresponding DDCS over the energy and emission angles of the ejected electron. Hence, the ionization cross section for the $i$-th subshell by an impinging proton with energy $E_{\mathrm{p}}, \sigma_{i}^{\text {ion }}\left(E_{\mathrm{p}}\right)$, is calculated using Equation (2.3.11) as

$$
\sigma_{i}^{\text {ion }}\left(E_{\mathrm{p}}\right)=\int \mathrm{d} E_{\mathrm{e}} \int \mathrm{d} \Omega_{\mathrm{e}} \frac{\mathrm{d}^{2} \sigma_{i}^{\text {ion }}}{\mathrm{d} E_{\mathrm{e}} \mathrm{d} \Omega_{\mathrm{e}}}\left(E_{\mathrm{p}} ; E_{\mathrm{e}}, \theta_{\mathrm{e}}\right) .
$$

For each energy of the proton and each subshell of the target element, the energy 
distribution of the ejected electron must be calculated using Equation (2.3.11). Then, calculations of the ionization cross section, see Equation (3.1.1), are performed for a few energies of the proton, $E_{\mathrm{p}}$, between 0.1 and $20 \mathrm{MeV}$. The integration over $\mathrm{d} E_{\mathrm{e}}$ implies that the range of the energy of the ejected electron must be defined in such a way that the integral is not underestimated. Here, the range in energy of the ejected electron $E_{\mathrm{e}}$ runs from $0.1 \mathrm{eV}$ to $1 \mathrm{MeV}$. For simplicity, these limits have been chosen to be the same for all shells and proton energies. These values are appropriate because the ionization DDCS highly increases on the lower energy limit, mainly for the outer shells, while its contribution from the K shell on the high energy limit is still significant.

In this way, the ionization DDCS in the BEA can be studied in terms of its sensitivity to the model of the VD of the electrons. The effects on the SEB and AB+RI DDCSs are discussed in Chapter 5.

\subsubsection{Comparison between the models for the VD}

As reviewed in Section 2.3.1.2, numerical solutions of the relativistic wave equation in the DHFS method provide more realistic VDs of the speed of the electrons, as opposed to the analytical solutions of the nonrelativistic wave equation for a pure Coulomb field. The DHFS PDFs take into account the relativistic effects, mainly important in the inner shells of atoms with higher values of the atomic number. Moreover, they account for the screening of the potential felt by the electrons in the outer shells of the atom. Therefore, it is interesting to compare both the DHFS and the hydrogenic VDs.

In order to calculate the DHFS PDFs, the radial wave functions $P_{n \kappa}(r)$ and $Q_{n \kappa}(r)$, which are solutions to the Equations (2.3.24), must be calculated. In this work, numerical calculations of $P_{n \kappa}(r)$ and $Q_{n \kappa}(r)$ and of the final VDs have been provided by J. M. FernándezVarea, employing the DHFS program developed at the University of Barcelona (Salvat and Fernández-Varea, 2019). This program consists of a FORTRAN subroutine package implemented by Salvat and Fernández-Varea (2019) in order to obtain numerical solutions of Dirac (relativistic) wave equations coupled to a DHFS self-consistent procedure.

More specifically, it contains the RADIAL package, developed to solve the radial equations for central potentials $V(r)$, such that $r V(r)$ is finite for all $r$ and tends to constant values when $r \rightarrow 0$ and $r \rightarrow \infty$. In the case of the DHFS method, in which the central potential is given by Equation (2.3.23), the code can be used to solve the Radial Equations (2.3.24), giving the radial wave functions $P_{n \kappa}(r)$ and $Q_{n \kappa}(r)$. The corresponding wave functions in the momentum space are obtained from Equations (2.3.25) and (2.3.26). Finally, the DHFS VDs can be calculated using Equation (2.3.27).

Alternatively, the wave functions in momentum space, for the first few values of the 
quantum numbers, are available in textbooks (Bransden and Joachain, 2003). However, these expressions become increasingly complex for the outer subshells. Therefore, in this work, the hydrogenic VDs are employed for the (sub)shells K, L, and M, while for the N and outer (sub)shells $(n \geq 4)$, the hydrogenic 1s VD (Equation (2.3.15)) is used.

The VDs have been calculated for aluminum, silver, and gold, and the results for the L subshells are shown in Figure 3.1, where $\beta$ is the speed of the electron divided by the speed of light. The dashed curves represent the hydrogenic VDs, while the solid curves show the DHFS VDs; different colors are assigned to each subshell. The binding energies of each subshell are listed in Table 3.3: note that the $\mathrm{L}_{2}$ and $\mathrm{L}_{3}$ subshells have similar values of the binding energies, both for aluminum and silver. This fact causes their corresponding hydrogenic VDs to be almost identical. On the other hand, the hydrogenic and the DHFS VDs display differences in shape. These differences occur because the potential felt by the electron in the L-shell is not Coulombian. Besides, in the case of gold, relativistic effects are no longer negligible and are considered in the DHFS approach.

Furthermore, the VDs of the $\mathrm{L}_{1}$ subshells present a dip, resultant of the node of the $2 \mathrm{~s}$ reduced radial wave function. The DHFS VDs appear to be shifted towards higher values of $\beta$, as compared to the hydrogenic ones. In particular, they exhibit larger probabilities in the high momentum tails, which have strong impact on the cross section results derived with them.

\subsubsection{Electron bremsstrahlung DDCS}

Equation (2.3.3) is an approximation for the electron bremsstrahlung production cross section, Equation (2.2.2), near the low energy limit $\hbar \omega \ll E_{\mathrm{e}}^{\prime}$. To study the validity of this approximation, Ishii et al. (1976) compared it to the values from the tables calculated by Tseng and Pratt (1971) with exact partial wave expansions in a realistic central atomic potential, for the case of an aluminum target, with $E_{\mathrm{e}}^{\prime}=5 \mathrm{keV}$ and $\hbar \omega=4 \mathrm{keV}$. From the comparison, they found an agreement within $10 \%$ of accuracy.

In order to confirm the result obtained by Ishii et al. (1976), Equation (2.3.3) and the tables by Tseng and Pratt (1971) are compared here for aluminum and gold and for electron kinetic energies of $E_{\mathrm{e}}^{\prime}=5$ and $20 \mathrm{keV}$. The results are presented in Figure 3.2, where the solid lines were obtained through Equation (2.3.3) and the dashed lines were interpolated from the reference tables (Kissel et al., 1981; Tseng and Pratt, 1971). The graphs also show the results for different emission angles $\theta_{\mathrm{br}}: 45^{\circ}$ (red lines), $75^{\circ}$ (green), 90 (blue) and $135^{\circ}$ (pink). It is clear that the maximum cross section values occur approximately at angles of $75^{\circ}$ and $90^{\circ}$ for the calculations by Tseng and Pratt (1971) and Equation (2.3.3) by Ishii et al. (1976), respectively.

An important feature visible in Figure 3.2 is that the values are most likely to agree for energies of the photon comparable to that of the electron, i.e. $\hbar \omega \approx E_{\mathrm{e}}^{\prime}$. The graphs get 
(a)

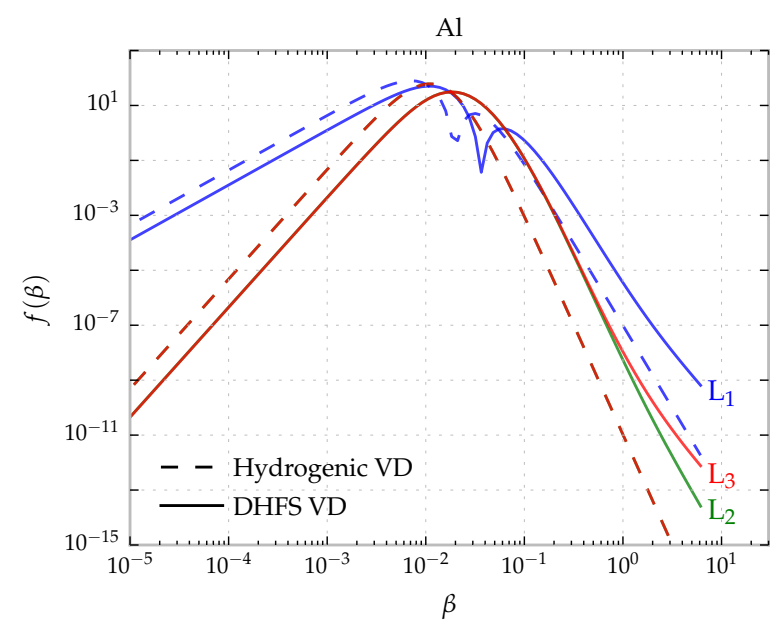

(b)

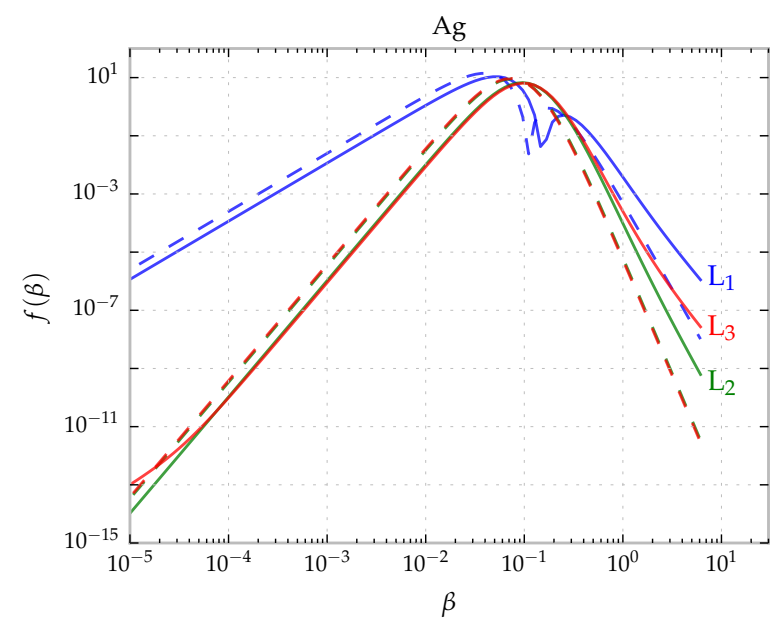

(c)

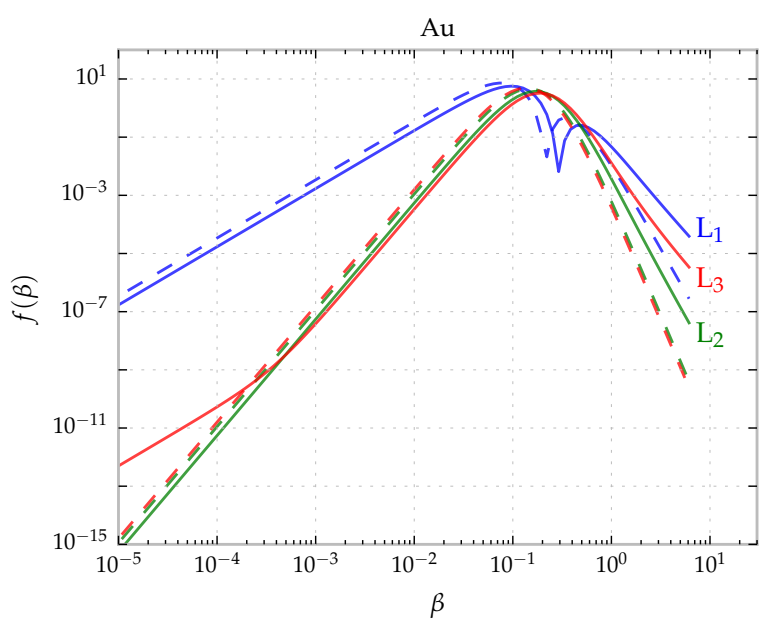

Figura 3.1: Hydrogenic VDs (dashed lines) and DHFS VDs (solid lines) for the L subshells of (a) $\mathrm{Al}$, (b) Ag, and (c) Au. The hydrogenic VDs of the $\mathrm{L}_{2}$ and $\mathrm{L}_{3}$ subshells of $\mathrm{Al}$ and $\mathrm{Ag}$ can not be distinguished in the figure since the respective binding energies are very similar.

further apart, though, for lower energies and are in large disagreement for the gold target with $E_{\mathrm{e}}^{\prime}=5 \mathrm{keV}$. These differences are expected, since Equation (2.3.3) does not include the effect of screening by the atomic electrons. Nevertheless, the equation is kept in the following approach to the SEB cross section, to allow for the analytic integration described in Section 3.1.5.

\subsubsection{Energy loss}

Equation (2.3.32) consists of an approximation to the electron energy loss derived by Bethe (1932), Equation (2.3.29). To verify the validity of this approximation, Equation (2.3.32) 
(a)

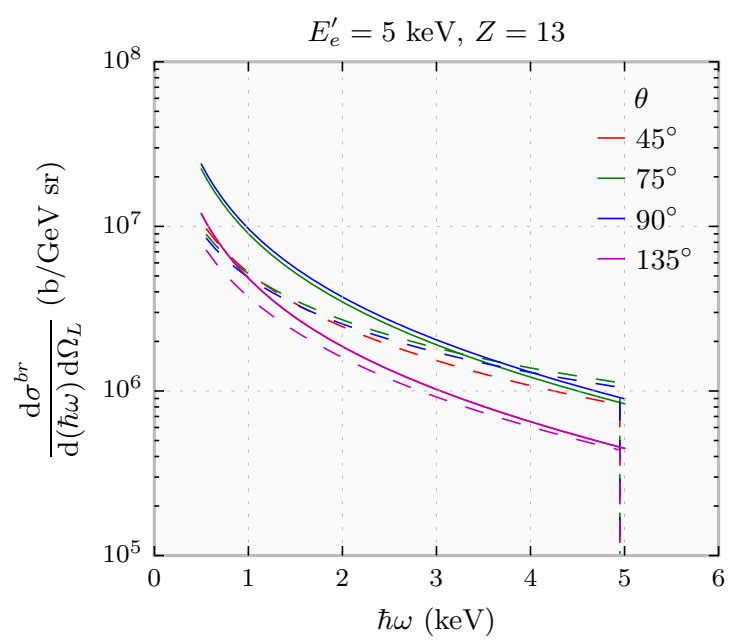

(c)

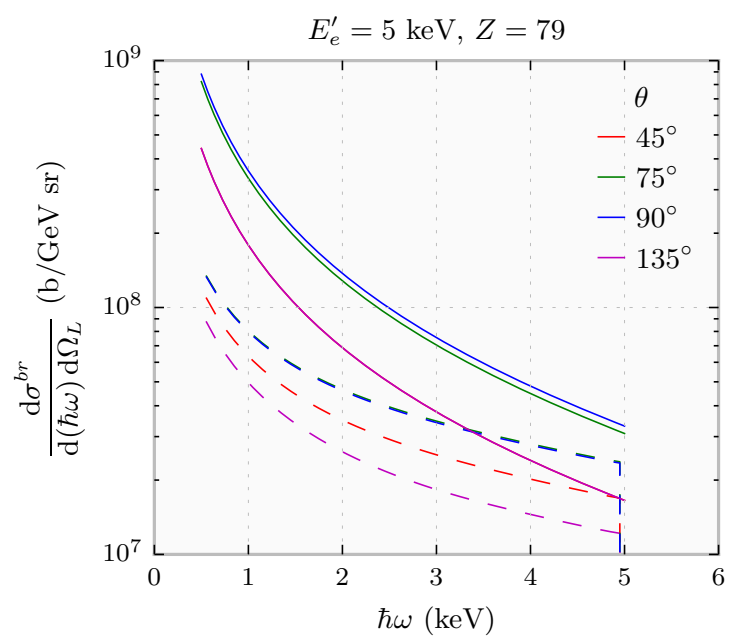

(b)

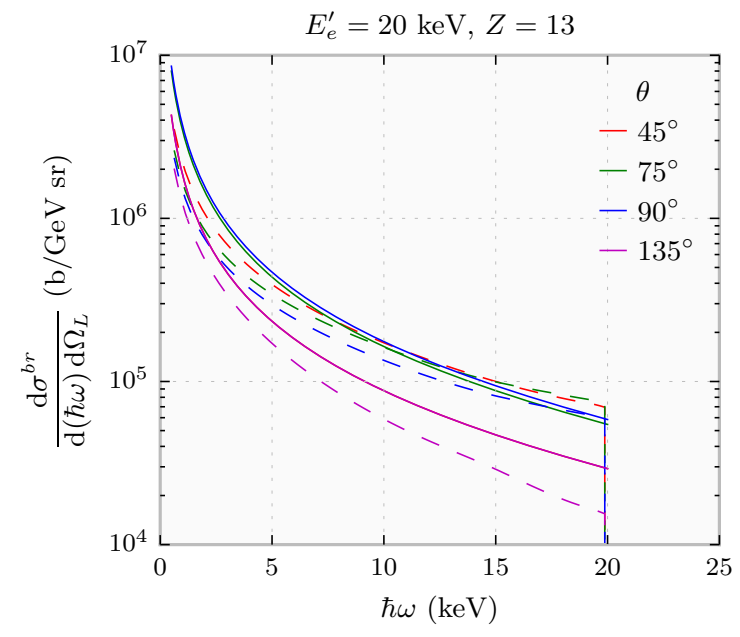

(d)

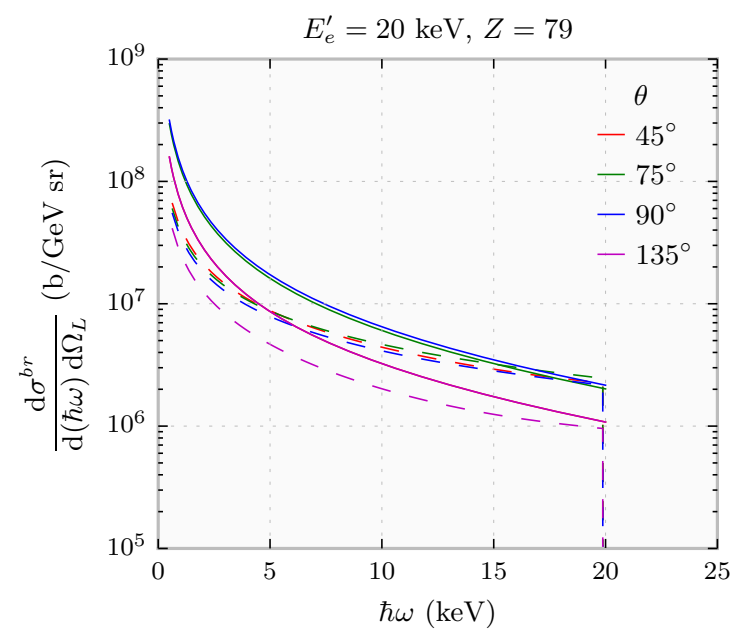

Figura 3.2: Electron bremsstrahlung production cross section. Comparison between Equation (2.3.3) (Ishii et al., 1976) (solid lines) and the published tables (Kissel et al., 1983; Tseng and Pratt, 1971) (dashed lines).

can be compared to values retrieved from the NIST ESTAR database (Berger et al., 2017). ESTAR consists of stopping power and range tables for electrons, based on the ICRU Report 37 (ICRU, 1984), which correspond to the use of an energy loss formula, more complete than Eqs. (2.3.30) and (2.3.31), accounting for the shell, Barkas, and density corrections. ESTAR includes 72 materials and electron kinetic energies ranging from $10 \mathrm{keV}$ to $1000 \mathrm{MeV}$. The results are illustrated for aluminum and gold in Figure 3.3. Besides the stopping force, $S_{\mathrm{f}}=-d E / d x$, the CSDA range values, $\Delta x$, defined by Equation (2.3.34), are also displayed.

A good agreement between Equation (2.3.32) and the database is visible, for both materials, up to energies of about $1 \mathrm{MeV}$. In this sense, when considering, for instance, an 
(a)

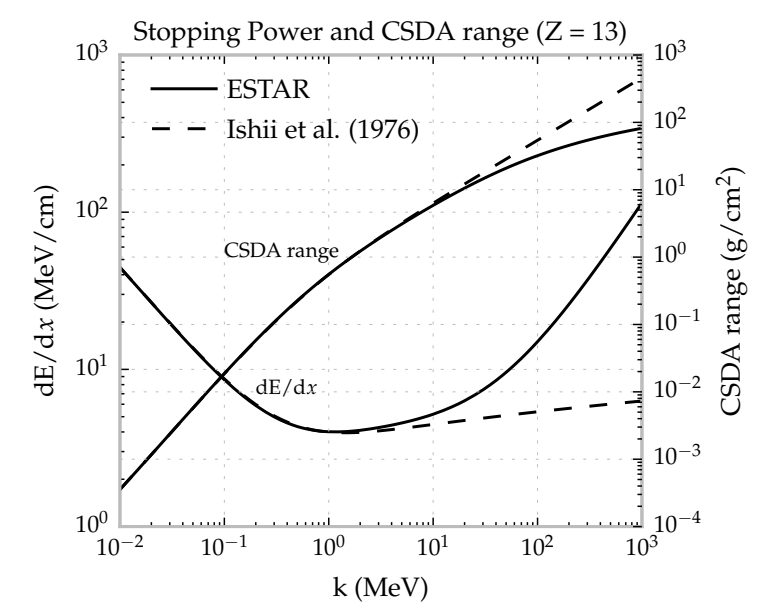

(b)

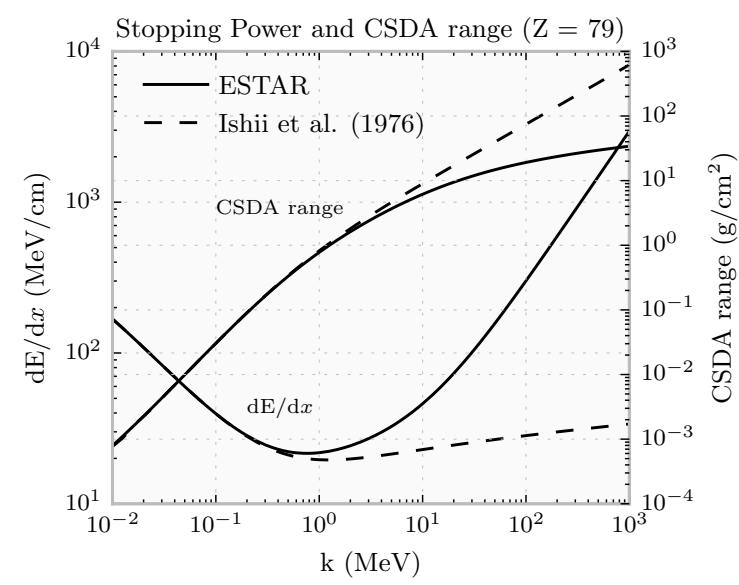

Figura 3.3: Comparison of the stopping power and CSDA range between the expression used by Ishii et al. (1976) (dashed lines) and the NIST ESTAR database (Berger et al., 2017) (solid lines), for (a) aluminum and (b) gold elements.

impinging proton with a kinetic energy of $E_{\mathrm{p}}=4 \mathrm{MeV}$, an elastic collision with a free electron can transfer a maximum amount of energy of $T_{\max }=4\left(m_{\mathrm{e}} / m_{\mathrm{p}}\right) E_{\mathrm{p}} \simeq 8.7 \mathrm{keV}$. Therefore, Equation (2.3.32) can be considered a good approximation to the electron energy loss in the SEB process for the energies of interest in this work.

\subsubsection{Calculation of the SEB DDCS}

With the results presented above about the expressions for the ionization DDCS, the bremsstrahlung DDCS, and the energy loss, it is possible to consider the numerical integration of all terms in Equation (2.3.2) in order to calculate the SEB DDCS. However, the integrals over $\mathrm{d} \varphi_{\mathrm{e}}$ and $\mathrm{d} E_{\mathrm{e}}^{\prime}$ can be evaluated analytically. This approach reduces the dimensions of the integral to be computed numerically from five to three, with a consequent gain in computation time. In particular, using Equations (2.3.3), (2.3.1), and (2.3.32), the terms involving the integrals over 
$\mathrm{d} \varphi_{\mathrm{e}}$ and $\mathrm{d} E_{\mathrm{e}}^{\prime}$ give

$$
\begin{aligned}
& \int_{0}^{2 \pi} \mathrm{d} \varphi_{\mathrm{e}} \int_{\hbar \omega}^{E_{\mathrm{e}}} \frac{\mathrm{d} E_{\mathrm{e}}^{\prime}}{S\left(E_{\mathrm{e}}^{\prime}\right)} \frac{\mathrm{d}^{2} \sigma_{\mathrm{br}}}{\mathrm{d}(\hbar \omega) \mathrm{d} \Omega_{\mathrm{br}}}= \\
&=\int_{0}^{2 \pi} \mathrm{d} \varphi_{\mathrm{e}} \int_{\hbar \omega}^{E_{\mathrm{e}}} \mathrm{d} E_{\mathrm{e}}^{\prime} \frac{v_{\mathrm{e}}^{\prime 2}}{4 \pi r_{0}^{2} Z m_{\mathrm{e}} c^{2}} \frac{2 Z^{2} \alpha r_{0}^{2}}{\pi v_{\mathrm{e}}^{\prime 2} \hbar \omega} \ln \left(4 \frac{E_{\mathrm{e}}^{\prime}}{\hbar \omega}\right)\left[\ln \left(\frac{1.16}{\bar{I}} E_{\mathrm{e}}^{\prime}\right)\right]^{-1}, \\
& \quad \times\left(1-\left(\cos \theta_{\mathrm{e}} \cos \theta+\sin \theta_{\mathrm{e}} \sin \theta \cos \varphi_{\mathrm{e}}\right)^{2}\right)= \\
&=A\left(2 \pi-2 \pi \cos ^{2} \theta_{\mathrm{e}}+3 \pi \cos ^{2} \theta_{\mathrm{e}} \sin ^{2} \theta-\pi \sin ^{2} \theta\right) \int_{1}^{E_{\mathrm{e}} / \hbar \omega} \mathrm{d} x \frac{\ln (a x)}{\ln (b x)}
\end{aligned}
$$

where $A=\alpha Z / 2 \pi^{2} m_{\mathrm{e}} c^{2} \mathcal{N}, a=4, b=1.16 \hbar \omega / \bar{I}$ and $x=E_{\mathrm{e}}^{\prime} / \hbar \omega$. The integral over $\mathrm{d} x$ in the above equation can be evaluated using the formula

$$
\int \mathrm{d} x \frac{\ln (a x)}{\ln (b x)}=\frac{[\ln (a x)-\ln (b x)] \operatorname{Ei}(\ln (b x))}{b}+x+\text { constant }
$$

where $\operatorname{Ei}(y)=-\int_{-y}^{\infty} e^{-t} / t \mathrm{~d} t$ is the exponential integral (Abramowitz and Stegun, 1970). Thereby, Equation (3.1.2) results in

$$
\begin{gathered}
\left.A\left(2 \pi-2 \pi \cos ^{2} \theta_{\mathrm{e}}+3 \pi \cos ^{2} \theta_{\mathrm{e}} \sin ^{2} \theta-\pi \sin ^{2} \theta\right)\left[\frac{\ln (a / b)}{b} \operatorname{Ei}(\ln (b x))+x\right]\right|_{1} ^{E_{\mathrm{e}} / \hbar \omega}= \\
=\frac{Z \alpha}{2 \pi m_{\mathrm{e}} c^{2} \hbar \omega}\left(2 \sin ^{2} \theta_{\mathrm{e}}+3 \cos ^{2} \theta_{\mathrm{e}} \sin ^{2} \theta-\sin ^{2} \theta\right)\left\{\frac{\bar{I}}{1.16} \ln \left(\frac{4 \bar{I}}{1.16 \hbar \omega}\right) \times\right. \\
\left.\times\left[\operatorname{Ei}\left(\ln \left(\frac{1.16}{\bar{I}} E_{\mathrm{e}}\right)\right)-\operatorname{Ei}\left(\ln \left(\frac{1.16}{\bar{I}} \hbar \omega\right)\right)\right]+E_{\mathrm{e}}-\hbar \omega\right\} .
\end{gathered}
$$

Finally, this result can be inserted into Equation (2.3.2) and the terms can be rearranged to give 
the following expressions

$$
\begin{aligned}
\frac{\mathrm{d}^{2} \sigma^{\mathrm{SEB}}}{\mathrm{d}(\hbar \omega) \mathrm{d} \Omega}= & \mathcal{N} \sum_{i} N_{i} \int_{\hbar \omega}^{E_{\mathrm{p}}-U_{i}} \mathrm{~d} E_{\mathrm{e}} \int_{0}^{\pi} \sin \theta_{\mathrm{e}} \mathrm{d} \theta_{\mathrm{e}} \int_{v^{\min }\left(E_{\mathrm{e}}, \theta_{\mathrm{e}}\right)}^{v^{\max }\left(E_{\mathrm{e}}, \theta_{\mathrm{e}}\right)} \mathrm{d} v f_{i}(v) \frac{\mathrm{d}^{2} \sigma^{\mathrm{e}}\left(E_{\mathrm{e}}, \theta_{\mathrm{e}}, v\right)}{\mathrm{d} E_{\mathrm{e}} \mathrm{d} \Omega_{\mathrm{e}}} \\
& \times \int_{0}^{2 \pi} \mathrm{d} \varphi_{\mathrm{e}} \int_{\hbar \omega}^{E_{\mathrm{e}}} \frac{\mathrm{d} E_{\mathrm{e}}^{\prime}}{S\left(E_{\mathrm{e}}^{\prime}\right)} \frac{\mathrm{d}^{2} \sigma_{\mathrm{br}}\left(E_{\mathrm{e}}^{\prime}, \hbar \omega, \theta_{\mathrm{br}}\right)}{\mathrm{d}(\hbar \omega) \mathrm{d} \Omega_{\mathrm{br}}}= \\
= & \sum_{i} N_{i} \int_{\hbar \omega}^{E_{\mathrm{p}}-U_{i}} \mathrm{~d} E_{\mathrm{e}} \int_{0}^{\pi} \sin \theta_{\mathrm{e}} \mathrm{d} \theta_{\mathrm{e}} \int_{v^{\max }\left(E_{\mathrm{e}}, \theta_{\mathrm{e}}\right)}^{\max \left(E_{\mathrm{e}}, \theta_{\mathrm{e}}\right)} \mathrm{d} v f_{i}(v) \frac{\mathrm{d}^{2} \sigma^{\mathrm{e}}\left(E_{\mathrm{p}}, v ; E_{\mathrm{e}}, \theta_{\mathrm{e}}\right)}{\mathrm{d} E_{\mathrm{e}} \mathrm{d} \Omega_{\mathrm{e}}} \\
& \times \frac{Z \alpha}{2 \pi m_{\mathrm{e}} c^{2} \hbar \omega}\left(2 \sin ^{2} \theta_{\mathrm{e}}+3 \cos ^{2} \theta_{\mathrm{e}} \sin ^{2} \theta-\sin { }^{2} \theta\right)\left\{\frac{\bar{I}}{1.16} \ln \left(\frac{4 \bar{I}}{1.16 \hbar \omega}\right)\right. \\
\times & \left.\left.\frac{Z Z_{\mathrm{p}}^{2} \alpha}{2 \pi^{2} m_{\mathrm{e}} c^{2} \hbar \omega} \sum_{i} N_{i}\left[\left(3 C_{2 i}-C_{1 i}\right) \sin ^{2} \theta+2\left(\frac{1.16}{\bar{I}} E_{\mathrm{e}}\right)\right)-\operatorname{Ei}\left(\ln \left(\frac{1.16}{\bar{I}} \hbar \omega\right)\right)\right]+E_{\mathrm{e}}-\hbar \omega\right\}
\end{aligned}
$$

with $C_{1 i}$ and $C_{21}$ given by

$$
\begin{aligned}
& C_{1 i}=\int_{\hbar \omega}^{E_{\mathrm{p}}-U_{i}} \mathrm{~d} E_{\mathrm{e}} \int_{0}^{\pi} \mathrm{d} \theta_{\mathrm{e}} \sin \theta_{\mathrm{e}} g_{i}\left(E_{\mathrm{p}} ; E_{\mathrm{e}}, \theta_{\mathrm{e}}\right), \\
& C_{2 i}=\int_{\hbar \omega}^{E_{\mathrm{p}}-U_{i}} \mathrm{~d} E_{\mathrm{e}} \int_{0}^{\pi} \mathrm{d} \theta_{\mathrm{e}} \cos ^{2} \theta_{\mathrm{e}} \sin \theta_{\mathrm{e}} g_{i}\left(E_{\mathrm{p}} ; E_{\mathrm{e}}, \theta_{\mathrm{e}}\right)
\end{aligned}
$$

and

$$
\begin{array}{r}
g_{i}\left(E_{\mathrm{p}} ; E_{\mathrm{e}}, \theta_{\mathrm{e}}\right)=\int_{v^{\min }\left(E_{\mathrm{e}}, \theta_{\mathrm{e}}\right)}^{v^{\max }\left(E_{\mathrm{e}}, \theta_{\mathrm{e}}\right)} \mathrm{d} v f_{i}(v) \times\left\{\frac{\bar{I}}{1.16} \ln \left(\frac{4 \bar{I}}{1.16 \hbar \omega}\right)\right. \\
\times\left[\operatorname{Ei}\left(\ln \left(1.16 \frac{E_{\mathrm{e}}}{\bar{I}}\right)\right)-\operatorname{Ei}\left(\ln \left(1.16 \frac{\hbar \omega}{\bar{I}}\right)\right)\right] \\
\left.\quad+E_{\mathrm{e}}-\hbar \omega\right\} \frac{\mathrm{d}^{2} \sigma^{\mathrm{e}}\left(E_{\mathrm{e}}, \theta_{\mathrm{e}}\right)}{\mathrm{d} E_{\mathrm{e}} \mathrm{d} \Omega_{\mathrm{e}}}\left(E_{\mathrm{p}}, v ; E_{\mathrm{e}}, \theta_{\mathrm{e}}\right) .
\end{array}
$$

The resulting Equation (3.1.5) contains integrations over $\mathrm{d} E_{\mathrm{e}}, \mathrm{d} \theta_{\mathrm{e}}$, and $\mathrm{d} v$, besides the 
exponential integral function. This expression has been implemented in the program along with Equation (2.3.4) (Yamadera et al., 1981) and the results are compared to the calculations by Ishii et al. (1976) in Section 3.3.

\subsection{Code implementation}

In order to study the main features of the proton bremsstrahlung mechanisms presented in Sections 2.3.1-2.3.4 and to reproduce the reference published results, a FORTRAN code has been developed to evaluate the DDCSs of each process. The program is designed to receive several input parameters and calculate the DDCSs as a function of the energy or the polar angle of the emitted photons for each bremsstrahlung process separately. To validate the results thus obtained, direct comparisons with the graphs shown in the reference articles have been made. In the following, a brief description of the program functionalities and usability is presented, along with minor details faced during the code implementation.

The numerical integrations are performed using the QUADPACK library, a set of FORTRAN 77 codes for numerical evaluation of integrals (Piessens et al., 1983). The package is part of the SLATEC Common Mathematical Library as a public domain software ${ }^{1}$. It is a solidly established and optimized code, being reimplemented in C language by the GNU Scientific Library $^{2}$ and interfaced in Python language through the Scipy library ${ }^{3}$ (Virtanen et al., 2020).

The QUADPACK library implements adaptive quadrature algorithms for one-dimensional functions. Specifically, the subroutines DQAG and DAQGI (Piessens et al., 1983) are applied, depending on the limits of integration being finite or infinite, respectively. The first one is an adaptive quadrature algorithm which allows the user to choose between 6 pairs of Gauss-Kronrod quadrature formulae as the basic rule. The second subroutine is designated to evaluate integrals over infinite intervals. In this case, the program analyses the integrand so that the infinite interval is mapped onto a finite one and an approach similar to the first routine is performed.

In both cases, the integrand function is specified to the subroutines with a FORTRAN symbol of type function, and must be defined together with the absolute or relative error tolerance. The program then adaptively selects the points where to perform the evaluation of the function, considering the requested precision on the final result.

Multi-dimensional problems can be approached by suitably combining the one-dimensional subroutines (Kahaner et al., 1989). In this case, the solution is to create multiple copies of the needed subroutine and perform nested calls to them. However, numerical errors must be

\footnotetext{
${ }^{1}$ The main repository for the library is http://www.netlib.org/

${ }^{2} \mathrm{https} / / / \mathrm{www} . \mathrm{gnu}$. org/software/gsl/

${ }^{3}$ https://www.scipy.org/
} 
handled accordingly. Nesting one-dimensional routines for adaptive quadrature in order to achieve multi-dimensional integrals requires the calculations of the inner integrals with higher precision (Kahaner et al., 1989). Consequently, as the number of nested integrals grows, the computation becomes less efficient.

A practical rule is to set the inner integral to be computed within an accuracy ten times greater than the outer one. Therefore, the relative tolerances are given by (Kahaner et al., 1989)

$$
\begin{aligned}
\epsilon_{o} & =0.9 \epsilon, \\
\epsilon_{i} & =\frac{\epsilon}{10(b-a)},
\end{aligned}
$$

where $\epsilon_{o}$ and $\epsilon_{i}$ are the outer and inner integrals relative tolerances, $[a, b]$ is the outer integral interval and $\epsilon$ is the input relative tolerance.

With the techniques discussed above, all proton bremsstrahlung processes have been properly implemented according to the corresponding references. The numerical implementation of the SEB DDCS is expected to be the most laborious, either due to the complexity of the equations derived by Yamadera et al. (1981) or due to the 5D integral needed for computing the generalized expression, defined in Equation (2.3.2). Moreover, the alternative formulation shown in Equation (3.1.5) is implemented as an alternative to Equation (2.3.4), because of the uncertainty about the probable misprints in the $g_{2}(s)$ function from Equation (2.3.8b).

A first difficulty in the evaluation of Equation (2.3.4) is represented by the discontinuity in the first derivative of the functions $g_{0}$ and $g_{2}$, see Equations (2.3.8), as it can be seen in the plots of Figure 3.4a. To circumvent this problem, numerical calculations must be performed in such a way that the integration limits correspond to the point of discontinuity of these functions. The subroutines for numerical quadrature are thus called separately for each interval.

Moreover, the function $g_{2}(s)$ requires attention when calculated numerically. It can be seen that this function tends to zero as $s \rightarrow \infty$. However, truncation errors due to limited decimal places of the floating point representation, an inherent characteristic of any computational approach, distort the behavior of the function making it strongly oscillating. The effects are shown in Figure 3.4b: the gray curve has been calculated with Mathematica ${ }^{\circledR}$ within machine precision (roughly 16 decimal digits), thus showing strong oscillations of the function for $s>1000$; the red curve was calculated with 32 decimal digits and represents the asymptotic behavior. Because the function is piecewise-defined, this numerical problem concerns only the upper part of the domain, for $\left|\frac{\gamma}{4}-1\right| \leq s \leq \infty$.

Direct numerical calculation of the $g_{2}(s)$ function with the double precision supported in hardware is therefore not always possible, then the problem is addressed as follows. 
(a)

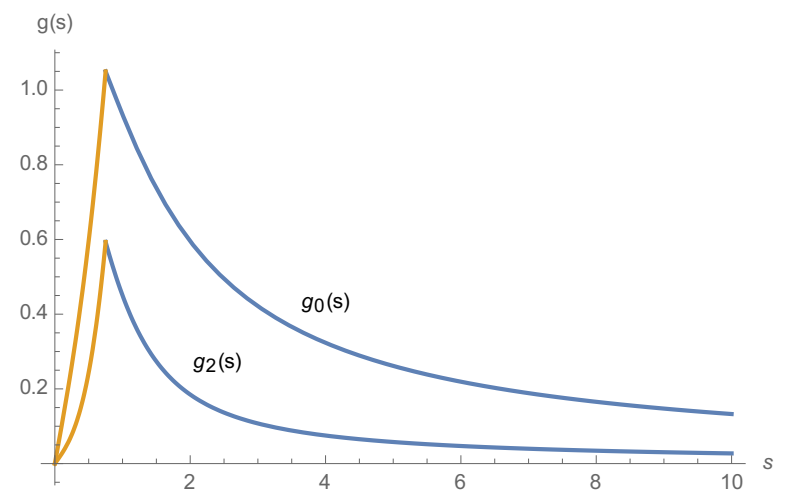

(b)

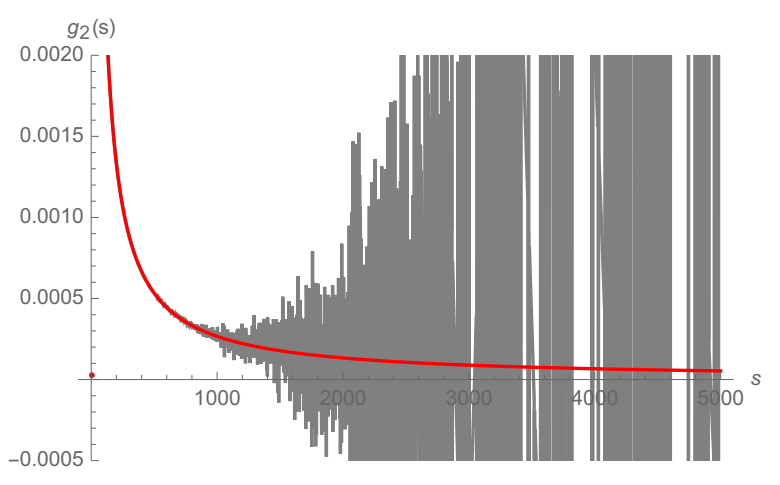

Figura 3.4: (a) Plots of the functions $g_{0}(s)$ e $g_{2}(s)$, for $\gamma=1$ and low values of $s$. (b) Plot of the function $g_{2}(s)$, with $s \geq\left|\frac{\gamma}{4}-1\right|$ and high values of $s$. The gray line was calculated with machine precision; the red line was calculated using 32 decimal digits of precision.

Firstly, the asymptotic expansion of $g_{2}(s)$, for $\left|\frac{\gamma}{4}-1\right| \leq s \leq \infty$, is calculated, which can be expressed as

$$
g_{2}(s) \approx \frac{4}{15 s}+\frac{2(7 \gamma+24)}{105 s^{3}}+\frac{\gamma^{3}}{48 s^{4}}+O\left(\left(\frac{1}{s}\right)^{6}\right)
$$

Its precision is estimated by comparing the terms at $O(3)$ and $O(4)$. This number is then compared to the input relative accuracy, $\epsilon_{\text {rel }}$, to establish whether the asymptotic expression is sufficient.

If the asymptotic expansion approach fails, the number of decimal digits for an accurate direct calculation of $g_{2}(s)$ is estimated, based on the values of $s, \gamma$, and the input relative tolerance, $\epsilon_{\text {rel }}$. If such value is greater than that available for double precision calculations, then an implementation of multiple precision arithmetics is employed. This is achieved through a FORTRAN package called MP (multiple-precision), develop by Brent (2010). The package consists of several routines that implement basic operations with arbitrary numerical precision, by representing each value as an array of integers. Therefore, the mathematical operations are re-implemented as functions and their return values are arrays. Consequently, the impact in computing time is noticeable, but the results are quite satisfactory.

The other approach to the integral involving the SEB process, discussed in Section 3.1, has also been implemented in the program through nested calls to QUADPACK library. The exponential integral is calculated numerically with the function DEXPIN (CERNLIB-C337), from the widely distributed CERNLIB library (Application Software Group, 1996).

The separate evaluation of the ionization DDCS in the BEA has also been implemented 
in the program, as discussed in Section 3.1.1. In this way, the ionization DDCSs can either be individually analyzed or used in the calculations of the SEB DDCS.

The AB+RI DDCSs can be calculated according to Equation (2.3.37), based on the BEA theory. Only a one dimensional integral over the VD is involved and is handled with a call to the QUADPACK library.

For the QFEB, the two versions, with and without the effect of the VD of the electrons in the target atom, see Equation (2.3.45) and (2.3.49), respectively, have been implemented. In the case of Equation (2.3.49), an integral over the PDF for the velocity component of the target electrons in the $z$ direction is performed with a call to the QUADPACK library. The calculations are first made within the reference frame of the proton. Then the Lorentz transformation to the laboratory frame is applied according to Equations (2.3.44).

Finally, for the NB, the implementation of Equation (2.3.50) does not require the evaluation of any integral.

For each process, besides the usual doubly differential cross section at fixed photon polar angle, different options are available. For example, the singly differential cross section or the power spectrum for a specific process can be requested. To give control over these variants, the program is intended to receive a set of input parameters specifying the process to be computed, the modes and types of calculation, and the physical parameters of the related formulae. This approach grants flexibility to perform any calculations within the validity of the implemented theories.

In practice, the program reads an input file with keyword arguments to set the parameters for the calculations. Table 3.1 details the input variables necessary for the program to be executed. The first group relates to the type of calculation, while the second one lists the physical variables. Note that not all these variables are necessary for all the processes. The programs automatically checks if all the needed information has been provided, once a specific bremsstrahlung process has been chosen. Because some variables depend on the atomic sub-shells, one input file per sub-shell is required. The VD of the electrons can also be read from an external file, if requested. Consequently, one output is generated for each sub-shell, which allows for their isolated contributions to be analyzed. 
Tabela 3.1: Input parameters for the program performing proton bremsstrahlung calculations.

\begin{tabular}{ll}
\hline Parameter & Description \\
ICAL & Type of calculation \\
IPRO & Proton bremsstrahlung process \\
MODI & Theoretical model control (specific cases) \\
PWR & Power spectrum required \\
DSDT & Cross section per polar angle required \\
RTOL & Relative accuracy of numerical integration \\
NELE & Energy loss model \\
\hline TPR & Proton kinetic energy \\
ZT & Target atomic number \\
NSHE & Number of electrons in the shell \\
NPB & Model of the VD of the shell \\
BSHE & Binding energy of the shell \\
IBET & Mean excitation energy of the target material \\
THE & Fixed photon angle \\
K & Fixed photon energy \\
KSC & Photon energy interval \\
TELS & Ejected electron kinetic energy range \\
TEL & Fixed ejected electron kinetic energy \\
TSC & Ejected electron angular range \\
\hline FNAM & Output filename \\
\hline
\end{tabular}

\subsection{Code validation}

In order to validate the FORTRAN code implementing the calculations of the DDCSs of the proton bremsstrahlung processes, several tests have been performed and the results have been compared to the corresponding cases available in the published works. Table 3.2 lists the particular reference used for benchmarking the program for each bremsstrahlung process.

In the processes where the VD of the electrons is necessary, namely $\mathrm{SEB}, \mathrm{AB}+\mathrm{RI}$, and QFEB, the 1s wave function has been used, so that the resulting DDCS can be compared with those from the references. The formula is given by Equation (2.3.15), where $v_{0}$ is related to the binding energy, $U_{i}$, of the corresponding atomic shell as $U_{i}=\frac{1}{2} m_{\mathrm{e}} v_{i}^{2}$. Particularly for the calculations of the QFEB DDCSs, the PDFs of the component of the velocity in the $z$ direction 
Tabela 3.2: Available theoretical references for benchmarking the program developed for the calculation of proton bremsstrahlung cross sections.

\begin{tabular}{llcccc}
\hline Process & Reference & Equation & Target material & Proton energy & Photon angle \\
\hline SEB & Ishii et al. (1976) & $(3.1 .5)$ & $\mathrm{Al}$ & 1 and $4 \mathrm{MeV}$ & $90^{\circ}$ \\
$\mathrm{AB}+\mathrm{RI}$ & Ishii et al. (2008) & $(2.3 .37)$ & $\mathrm{Al}, \mathrm{Ag}, \mathrm{Au}$ & $1.5 \mathrm{MeV}$ & $90^{\circ}$ \\
QFEB & Chu et al. (1981) & $(2.3 .49)$ & $\mathrm{Be}$ & $20 \mathrm{MeV}$ & $90^{\circ}$ \\
QFEB & Chu et al. (1981) & $(2.3 .45)$ & $\mathrm{Be}$ & $20 \mathrm{MeV}$ & $50^{\circ}, 90^{\circ}, 130^{\circ}$ \\
NB & Ishii et al. (1999) & $(2.3 .50)$ & $\mathrm{Al}$ & $1.5 \mathrm{MeV}$ & $45^{\circ}, 90^{\circ}$ \\
\hline
\end{tabular}

Tabela 3.3: Some reference values of the binding energy ${ }^{4}$, in $\mathrm{eV}$, for each atomic shell, used in the calculations of $A B+R I, Q F E B$, and SEB cross sections. Not all values are available.

\begin{tabular}{|c|c|c|c|c|c|c|c|c|c|c|c|c|c|}
\hline \multirow[b]{2}{*}{ Z } & \multicolumn{13}{|c|}{ Binding energy $(\mathrm{eV})$} \\
\hline & K & L1 & $\mathrm{L} 2$ & L3 & M1 & M2 & M3 & M4 & M5 & $\mathrm{N} 1$ & $\mathrm{~N} 2$ & $\mathrm{~N}$ & \\
\hline 4 & 111.5 & & & & & & & & & & & & \\
\hline 13 & 1559.6 & 117.8 & 72.95 & 72.55 & & & & & & & & & \\
\hline 47 & 25514 & 3806 & 3524 & 3351 & 719 & 603.8 & 573 & 374 & 368.3 & 97 & 63.7 & 58. & 3.3 \\
\hline 79 & 80725 & 14353 & 13734 & 11919 & 3425 & 3148 & 2743 & 2291 & 2206 & 762.1 & 642 & $7 \quad 546$ & \\
\hline & & & & & & & & & (Cont & inuatio & & & \\
\hline & & & & & & $\mathrm{Z}$ & $\mathrm{N} 4$ & N5 & N6 & N7 & O1 & $\mathrm{O} 2$ & $\mathrm{O} 3$ \\
\hline & & & & & & 79 & 353.2 & 335.1 & 87.6 & 84.0 & 107.2 & 74.2 & 57.2 \\
\hline
\end{tabular}

for the 1s and 2s orbitals (Equation (2.3.48)) are considered in order to allow a comparison with the results by Chu et al. (1981). Table 3.3 lists the reference values of the binding energy used in the calculations presented herein. However, because not all values are available for all elements, the practical procedure has been to allocate all the remaining electrons in one last shell with a binding energy $10 \%$ lower than the smaller one available. This method results in good agreement with the references.

The following subsections show the results for each process in comparison with the corresponding references. Additionally, the calculations of the ionization DDCSs in the BEA are discussed separately from the SEB or AB+RI DDCSs, and the results are compared to the calculations by Ishii et al. (1976), Bonsen and Vriens (1970), and J. M. Fernández-Varea.

\footnotetext{
${ }^{4}$ Source: http://xdb.lbl.gov/Section1/Table_1-1.pdf. Accessed on March, 2018.
} 
(a)

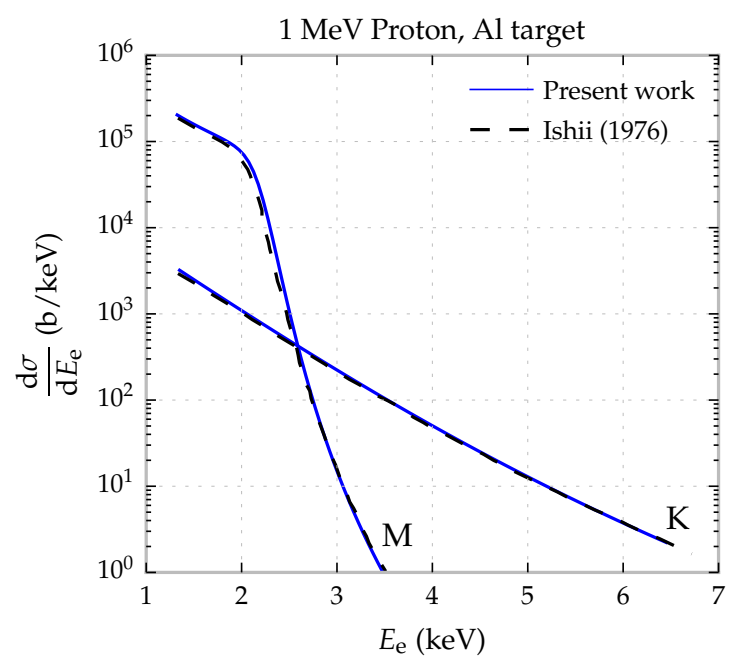

(b)

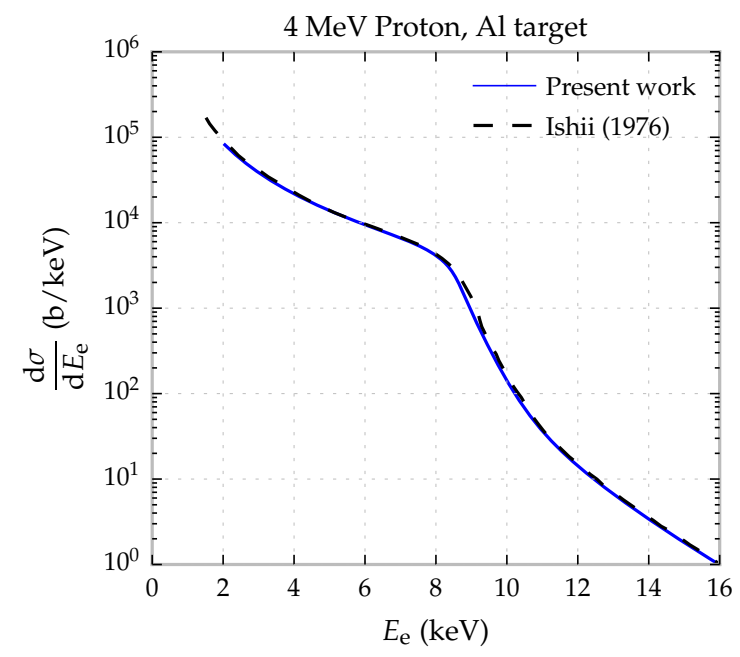

Figura 3.5: Energy distribution of ejected electrons from an aluminum target due to the impact of protons with energies of (a) $1 \mathrm{MeV}$ and (b) $4 \mathrm{MeV}$. Figure (a) shows the contributions of $\mathrm{K}$ and $M$ shells separately, and Figure (b) shows the total cross section. The dashed lines are the reference values (Ishii et al., 1976) and the solid lines have been calculated with the program developed in this work.

\subsubsection{Ionization DDCS}

As a first step, the theoretical values obtained by Ishii et al. (1976) have been employed to validate the program adopting the 1s hydrogenic VD, Equation (2.3.15). Figure 3.5 shows the results for an aluminum target, with (a) $E_{\mathrm{p}}=1$ and (b) $4 \mathrm{MeV}$. In the first panel, the contributions for the $\mathrm{K}$ and $\mathrm{M}$ shells are shown separately, while the second panel represents the total differential cross section, summing up the contributions from all shells. The plots show a very good agreement with the reference values.

Moreover, the results obtained by Bonsen and Vriens (1970) have also been compared. In this case, the angular distribution of the electrons, ejected from an helium atom by a proton with an energy of $300 \mathrm{keV}$, has been calculated for several ejection energies in Figure 3.6. These calculations also display a good agreement with the reference values.

Finally, another validation of the total cross section has been performed by comparing the results with another program developed by J. M. Fernández-Varea, which implements the formulae derived by Catlow and McDowell (1967). In their approach, the integrals over the energy and the angle are obtained analytically, and only the integral over the VD must be calculated numerically. The results by J. M. Fernández-Varea utilize the hydrogenic VDs, so the same has been done in the present work to allow a direct comparison. The calculations have been carried out for aluminum, silver, and gold targets, with energies ranging from 0.1 


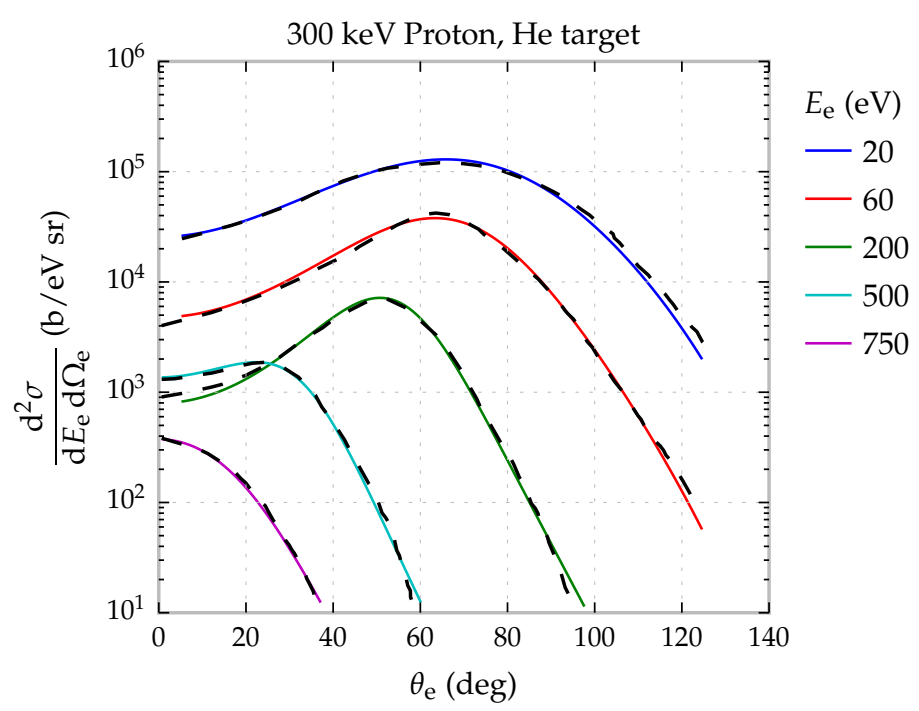

Figura 3.6: Angular distribution of ejected electrons from an helium atom due to the impact of $300 \mathrm{keV}$ protons. Several energies of the ejected electron are considered. The dashed lines are the reference values (Bonsen and Vriens, 1970) and the solid lines have been calculated with the program developed in this work.

to $5 \mathrm{MeV}$, as shown in Figure 3.7. The results are displayed for each shell, summing the cross sections obtained for the corresponding subshells. It can be noted that the two methods present a very good agreement.

\subsubsection{Secondary Electron Bremsstrahlung DDCS}

Calculations of the SEB DDCS were performed for an aluminum target and proton energies of 1 and $4 \mathrm{MeV}$. Figure 3.8 shows the results obtained from Equation (3.1.5) (blue lines) in comparison to the results from Equation (2.3.4) (red lines). Moreover, the dashed lines correspond to the values obtained numerically by Ishii et al. (1976). The results from Equation (2.3.4) by Yamadera et al. (1981) are about one order of magnitude lower than the previously published ones by the same group (Ishii et al., 1976). However, as discussed in the beginning of the chapter, it has to be noted that there are some uncertainties about the expressions involved in this formulation, possibly due to misprints in the cited articles (Ishii, 2006; Yamadera et al., 1981).

On the contrary, the formulae derived in Section 3.1 are consistent with the results obtained by Ishii et al. (1976). Therefore, Equation (3.1.5) has been used in all further calculations of the SEB DDCS for comparisons with the experiments performed at LAMFI (see Section 5.2). Besides, it has been noted that the computation times of Equation (3.1.5) are much lower than those of Equation (2.3.4). 
(a)

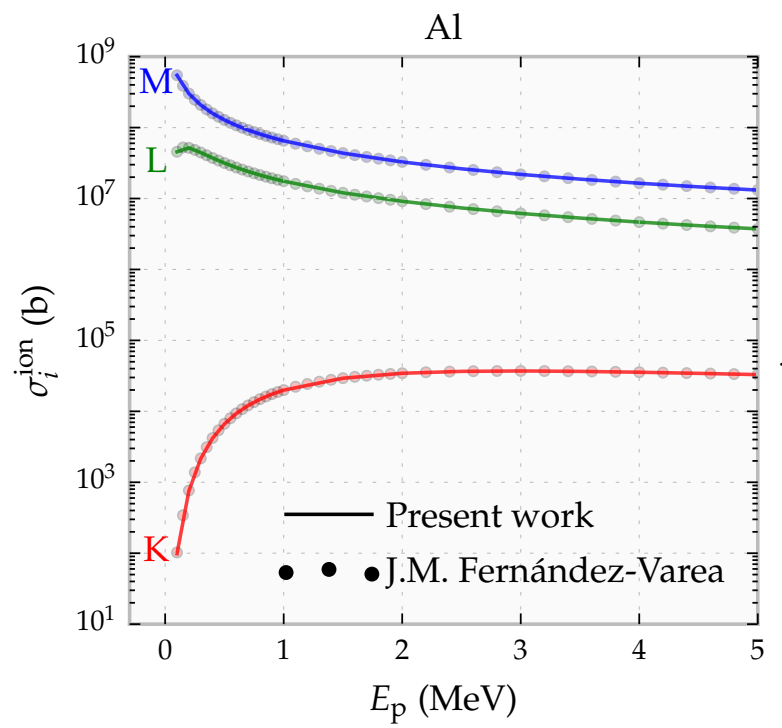

(b)

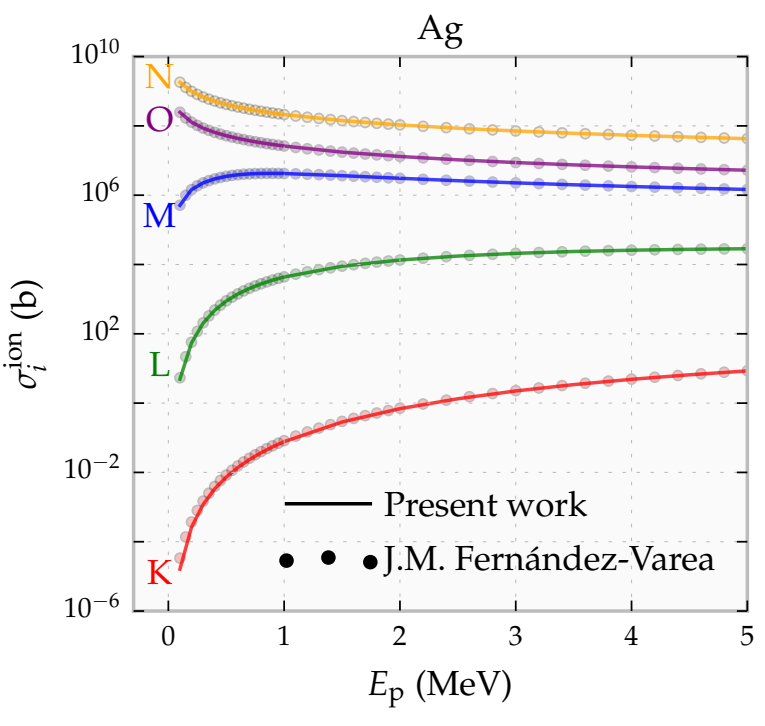

(c)

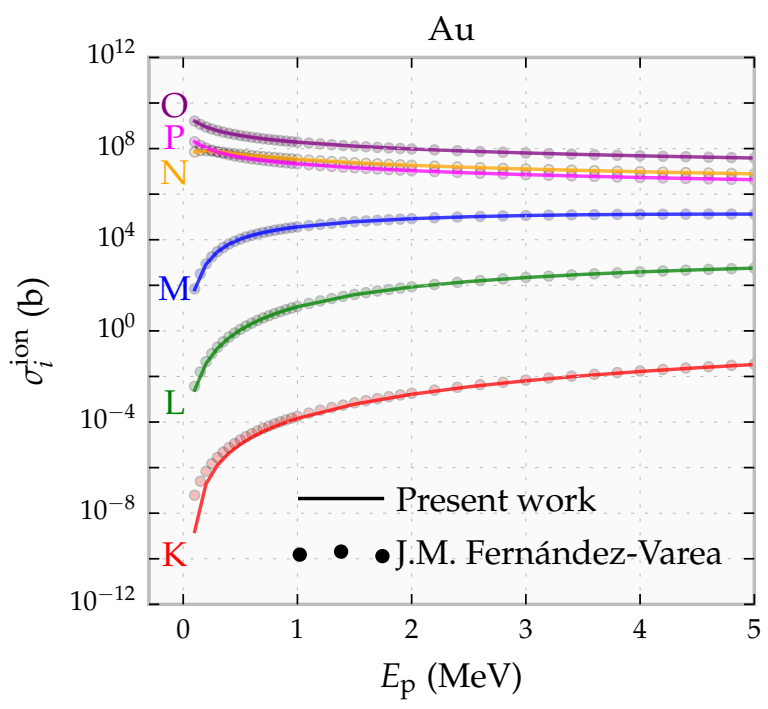

Figura 3.7: Ionization cross sections for (a) $\mathrm{Al}$, (b) $\mathrm{Ag}$, and (c) $\mathrm{Au}$, for each shell, comparing the results obtained by J. M. Fernández-Varea (points), using the formulae by Catlow and McDowell (1967), and the calculations performed in the present work (solid lines). Both cases implement the hydrogenic VDs.

\subsubsection{Atomic Bremsstrahlung and Radiative Ionization DDCS}

Calculations of the AB+RI DDCS have been performed for aluminum, silver, and gold targets and a proton energy of $1.5 \mathrm{MeV}$ (see Equation (2.3.37)). The program has been run for each atomic subshell and then the outputs summed up to give the total DDCS. Each subshell is characterized by a value of the binding energy which enters into the VD of the electrons (see Equation (2.3.15)). The results are displayed in Figure 3.9 with a pink line, while the dashed 
(a)

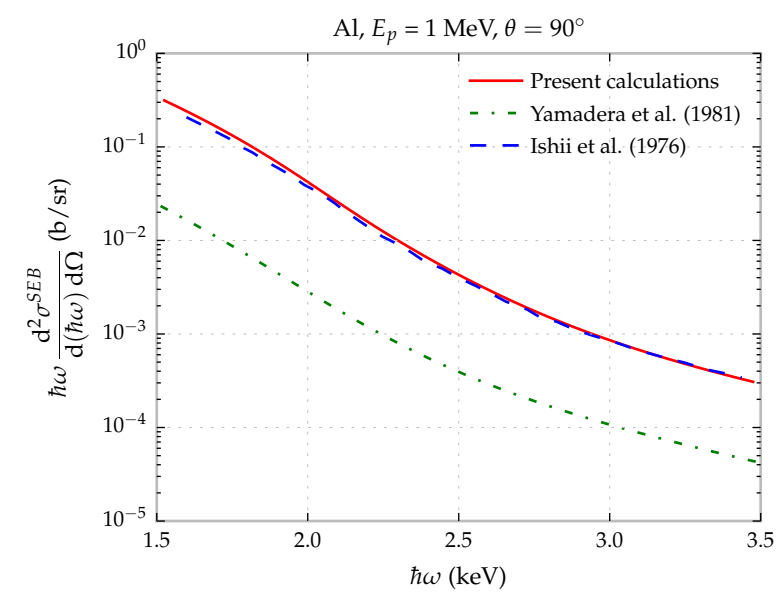

(b)

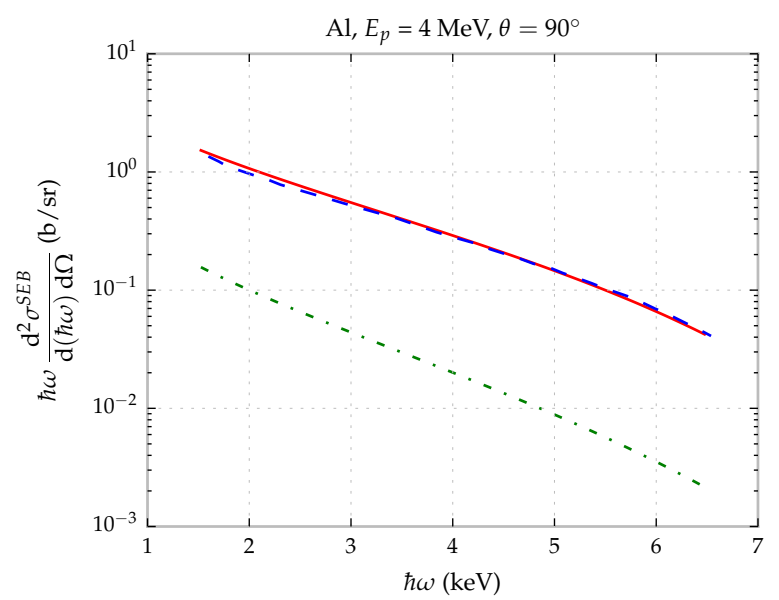

Figura 3.8: Calculations of the SEB cross sections for an aluminum target, proton energies of (a) $1 \mathrm{MeV}$ and (b) $4 \mathrm{MeV}$, and a photon emission angle of $90^{\circ}$. The blue lines correspond to the approach proposed in the present work, see Equation (3.1.5); the red lines correspond to the analytical expression obtained by Yamadera et al. (1981), see Equation (2.3.4). The dashed lines represents the numerical results obtained by Ishii et al. (1976).

line represents those obtained by Ishii et al. (2008). It is notable that a very accurate agreement could be achieved in these cases. The comparison also indicates that the values of the binding energy from Table 3.3 must be close to the ones used by Ishii et al. (2008).

\subsubsection{Quasi-Free Electron Bremsstrahlung DDCS}

Calculations of the QFEB DDCS have been performed for a beryllium target and a proton energy of $20 \mathrm{MeV}$. In this case, the relatively high energy of the proton allows to produce more energetic photons, that could be easily detected by Chu et al. (1981). Low energy protons, as employed for the measurements shown in Section 5.2, result in a contribution of the QFEB at very small photon energies (see Table 2.2), making it difficult to be measured.

Note that the reference article (Chu et al., 1981) presents the results as a power spectrum. That implies the multiplication of the cross section by $\hbar \omega$, which is easily performed by the program.

Figure 3.10 shows the results for Equation (2.3.45), without the effect of the PDF of the component of the velocity in the $z$ direction of the atomic electrons, for emission angles of (a) $50^{\circ}$, (b) $90^{\circ}$ and (c) $130^{\circ}$. The dashed lines represent the published values ${ }^{5}$ (Chu et al., 1981). In all these cases, the results are in reasonable good agreement with the reference.

Figure 3.11 shows the results for Equation (2.3.49), which considers the integration

\footnotetext{
${ }^{5}$ These lines appear to be noisy due to difficulties in scanning the original plots.
} 
(a)

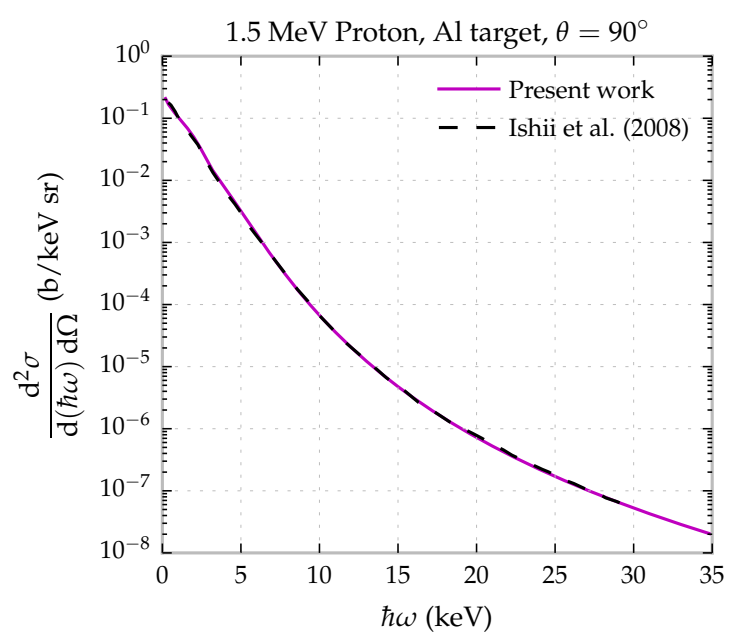

(c)

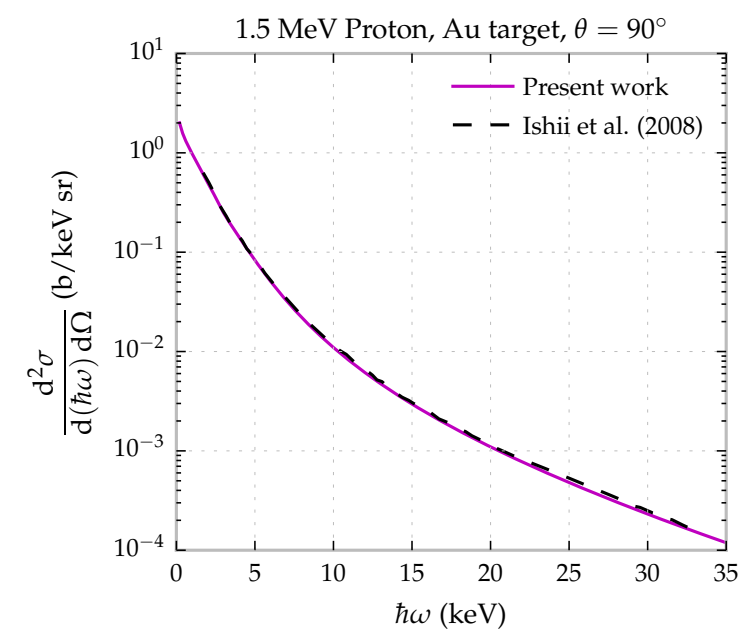

(b)

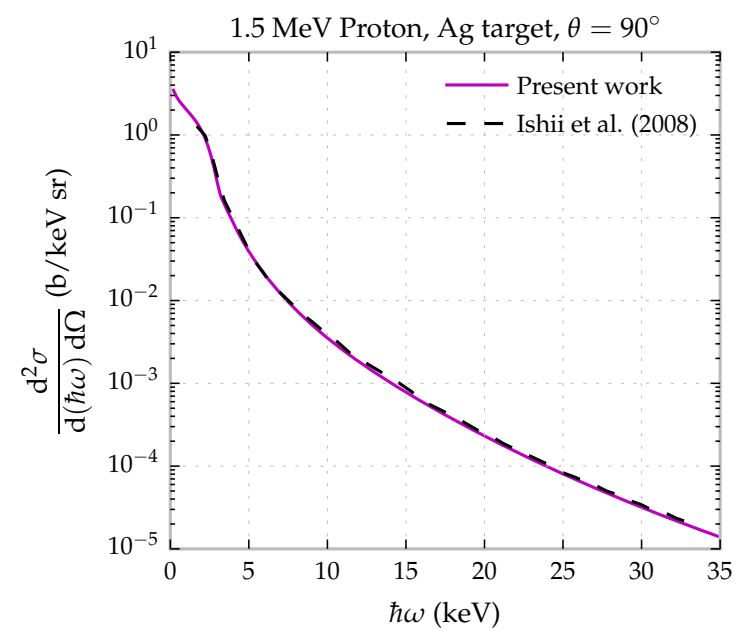

Figura 3.9: Calculations of the $A B$ cross sections for (a) aluminum, (b) silver and (c) gold targets, 1.5 MeV protons, and a photon emission angle of $90^{\circ}$. The pink lines correspond to the present implementation of Equation (2.3.37) and the dashed ones represent the theoretical results obtained by Ishii et al. (2008).

over the $z$ component (along the beam direction) of the velocity, for a photon emission angle of $90^{\circ}$. In this case, two approaches for the PDFs of the velocity component in the $z$ direction of the atomic electrons are considered. The plot in Figure 3.11(a) has been calculated with the 1s hydrogenic PDF of the velocity component in the $z$ direction, Equation (2.3.48a), for both the K and L shells. On the other hand, the graph in Figure 3.11(b) has been obtained by applying the 1s wave function, as in the first case, to K shell only, and the 2s PDF of the electron velocity 
(a)

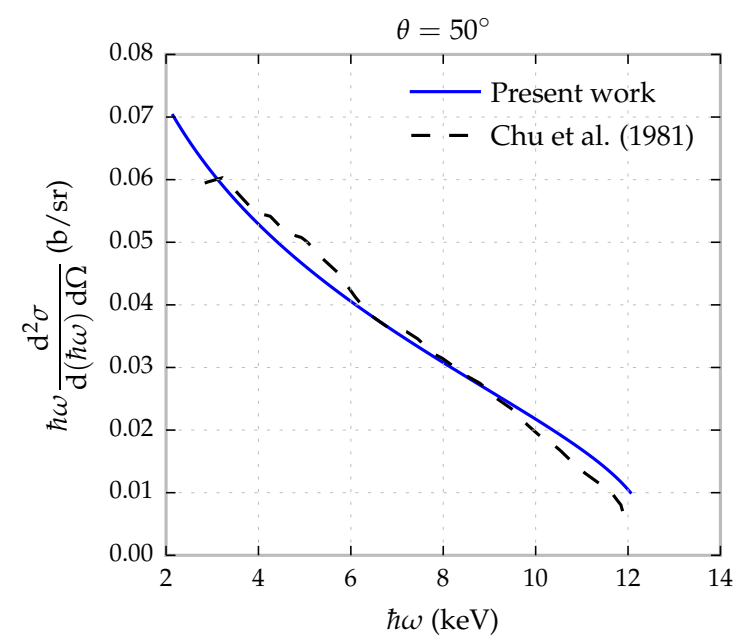

(c)

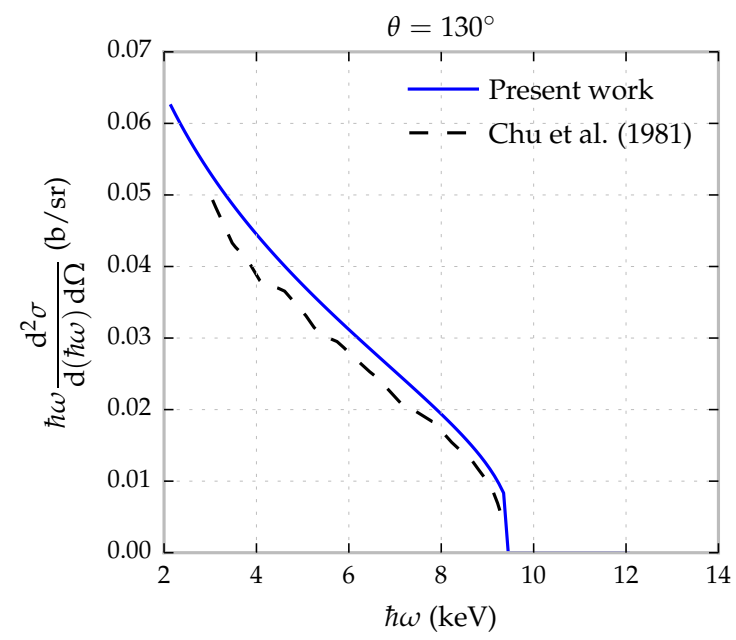

(b)

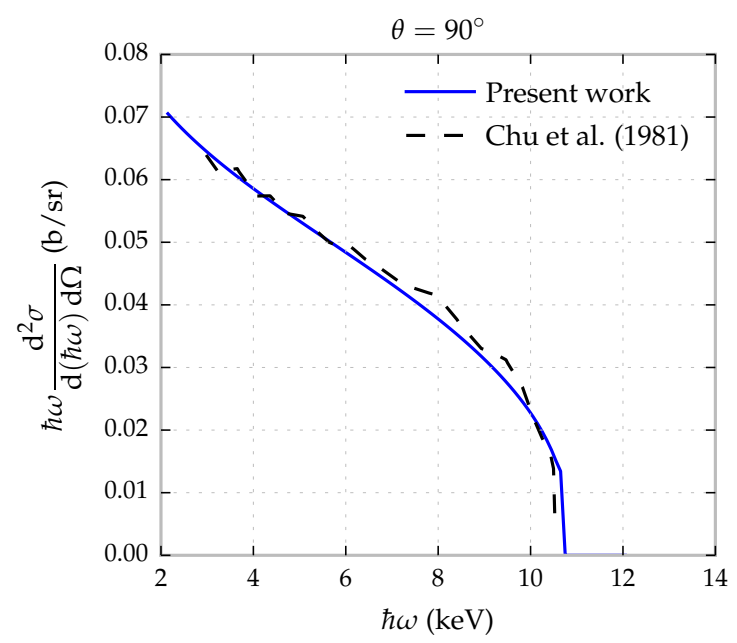

Figura 3.10: Calculations of the QFEB cross section for a beryllium target, $20 \mathrm{MeV}$ protons, and photon emission angles of (a) $50^{\circ}$, (b) $90^{\circ}$, and (c) $130^{\circ}$. The solid lines correspond to Equation (2.3.45) without the PDFs of the velocity component in the $z$ direction of the atomic electrons. The dashed lines represent the reference values (Chu et al., 1981).

in the $z$ direction, Equation (2.3.48b), to the $\mathrm{L}$ shell. This is different from the validation of the SEB and $\mathrm{AB}+\mathrm{RI}$ calculations, where only the 1s hydrogenic VD has been tested. Although the reference paper (Chu et al., 1981) mentions the use of both functions, the first situation, with calculations for one function only, appears to be in reasonably better agreement with the reference values. The results demonstrate the importance of taking into account the PDF of the velocity component in the $z$ direction of the orbital electrons in the evaluation of QFEB DDCS. 
(a)

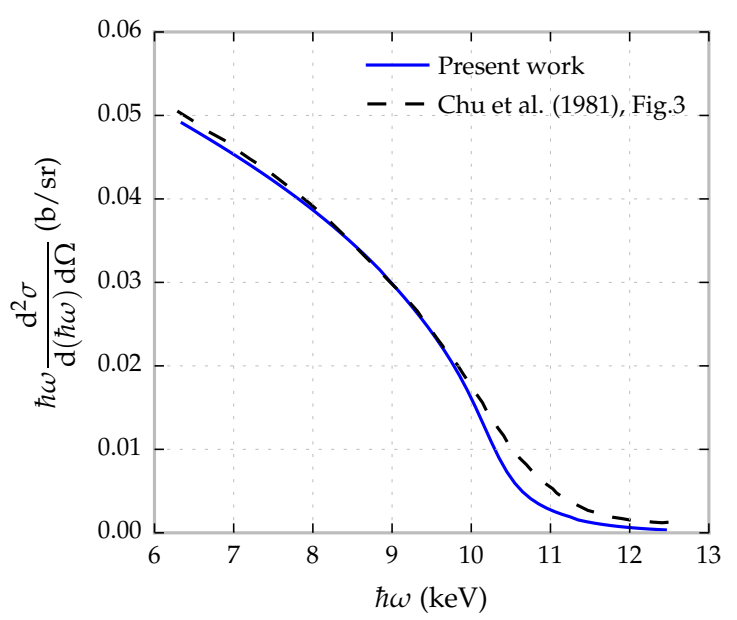

(b)

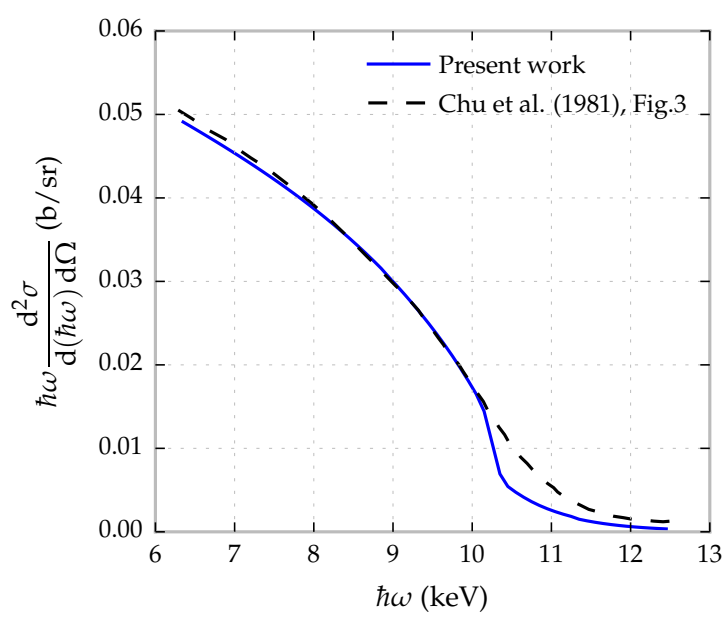

Figura 3.11: Calculations of the QFEB cross section for a beryllium target, $20 \mathrm{MeV}$ protons, and a photon emission angle of $90^{\circ}$. The solid lines correspond to Equation (2.3.49) with the PDFs of the velocity component in the $z$ direction of the atomic electrons. The following cases are considered: (a) $f_{1 s}\left(v_{z}\right)$ (Equation (2.3.48a)) applied for both K and L shells; (b) $f_{1 s}\left(v_{z}\right)$ and $f_{2 s}\left(v_{z}\right)$ (Equation (2.3.48b)) applied for K and L shells, respectively. The dashed lines represent the reference values (Chu et al., 1981).

\subsubsection{Nuclear Bremsstrahlung DDCS}

NB DDCS calculations have been performed for an aluminum target, with a proton energy of $1.5 \mathrm{MeV}$, and photon emission angles of $45^{\circ}$ and $90^{\circ}$, according to Equation (2.3.50). Figure 3.12 shows the results obtained for (a) $45^{\circ}$ and (b) $90^{\circ}$, in comparison to the reference values (dashed lines) calculated by Ishii et al. (1999). The program is in very good agreement with the reference. 
(a)

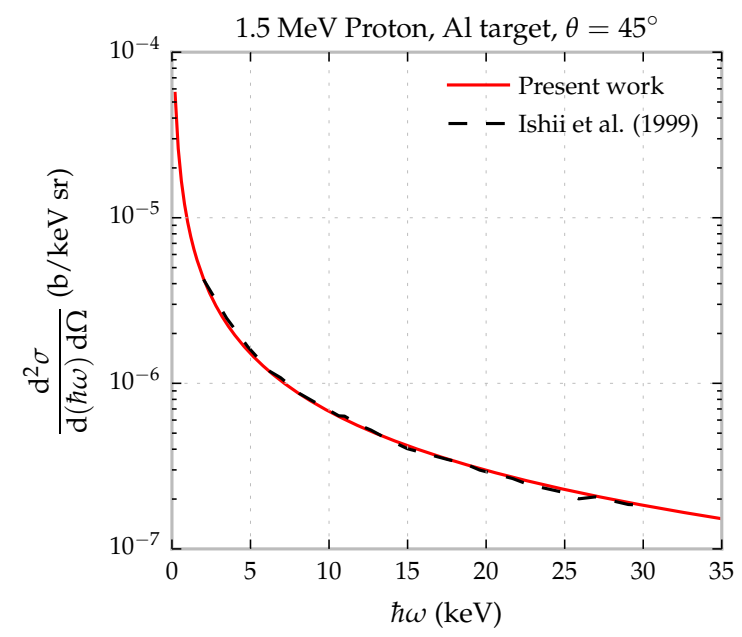

(b)

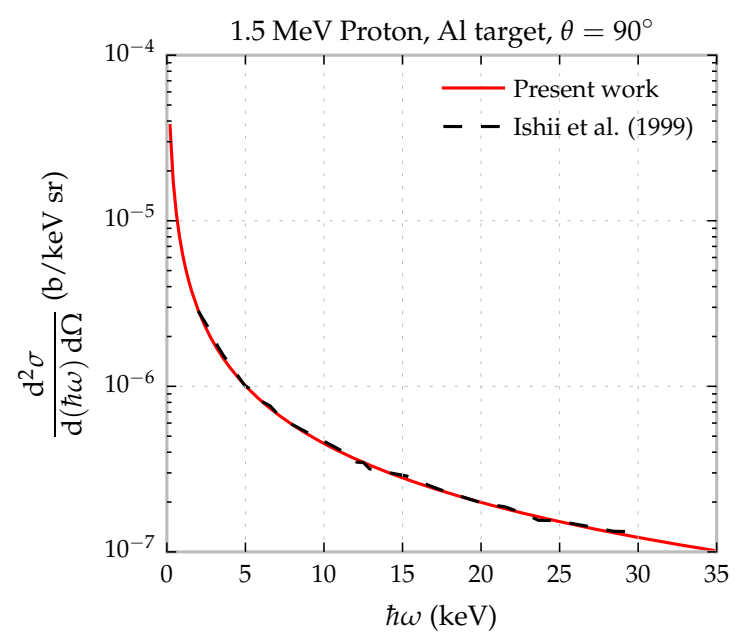

Figura 3.12: Calculations of the $\mathrm{NB}$ cross sections for an aluminum target, $1.5 \mathrm{MeV}$ protons, and photon emission angles of (a) $45^{\circ}$ and (b) $90^{\circ}$. The red lines correspond to Equation (2.3.50) and the dashed ones represent the theoretical results obtained by Ishii et al. (1999).

\section{Summary}

In order to study the main features of the proton bremsstrahlung DDCSs and to reproduce the results presented in the reference publications, a FORTRAN code has been developed to implement the calculations. The program reads an input file with keywords specifying the process to be computed, the modes and types of the calculation, and the physical parameters needed in the related formulae. Because some variables depend on the atomic sub-shells, one input file per sub-shell is required. Table 3.1 lists the input variables necessary for the program to be executed.

All proton bremsstrahlung processes have been properly implemented according to the corresponding references. The analytical expressions of the SEB cross section (Yamadera et al., 1981), however, could not be employed to reproduce the results published by the same group. In this case, a different approach has been suggested, resulting in the implementation of the SEB cross section as given by Equation (3.1.5). Moreover, some aspects of the SEB formula have been analyzed in more detail, such as the expressions of the electron bremsstrahlung and ionization cross sections, and the energy loss.

Table 3.2 lists the content of the references used for benchmarking the program for each bremsstrahlung process. One important point in common to these calculations is the integration over the electron VD, for which the analytic formula for the $1 \mathrm{~s}$ orbital has been applied to all atomic shells. 
Finally, the values obtained from the program (solid colored lines) and the reference ones from the corresponding papers (dashed lines) are compared in a series of plots. In all cases, a good agreement can be seen. 



\section{Capítulo 4}

\section{Experimental measurements}

Measurements of the x-ray spectrum induced by the proton impact have been performed at LAMFI, Laboratório de Análise de Materiais e Feixes Iônicos, at the University of São Paulo. The experiment has been carried out by T. F. da Silva, obtaining two PIXE spectra for a gold target and proton energies of 2 and $3 \mathrm{MeV}$. In this way, the theoretical models for proton bremsstrahlung, reviewed in Chapter 2, can be analyzed in comparison to the experiments. Moreover, the proposed expression for the SEB process, presented in Chapter 3, can be validated, and the effects of the implementation of the DHFS VDs in the SEB DDCS can be studied in comparison to data.

Nonetheless, the comparison of the calculated DDCSs for proton bremsstrahlung with data must take into account the detection effects. More precisely, the analysis of the experiment requires knowledge of the detector, namely its efficiency and response function, and the electronic chain, to describe the pile-up effect, besides the effects of self attenuation of the photon in the target medium. The model of the response function of the detector and the calibration of the parameters involved have been performed with the help of T. F. da Silva. In this work, the detection effects are applied to the theoretical calculations, thus allowing a quantitative comparison with the measured PIXE spectra, see Chapter 5.

The experimental aspects of the work are discussed in the present Chapter. Section 4.1 presents the experimental arrangement used at LAMFI to measure the PIXE spectra. The detection effects are reviewed in Section 4.2 and the method adopted to determine the response function of the detector is discussed in Section 4.3. Additionally, a method for estimating a suitable thickness of a target, mainly for studying the SEB process, is discussed in Section 4.4, as a remark for future experiments that might come to interest. 


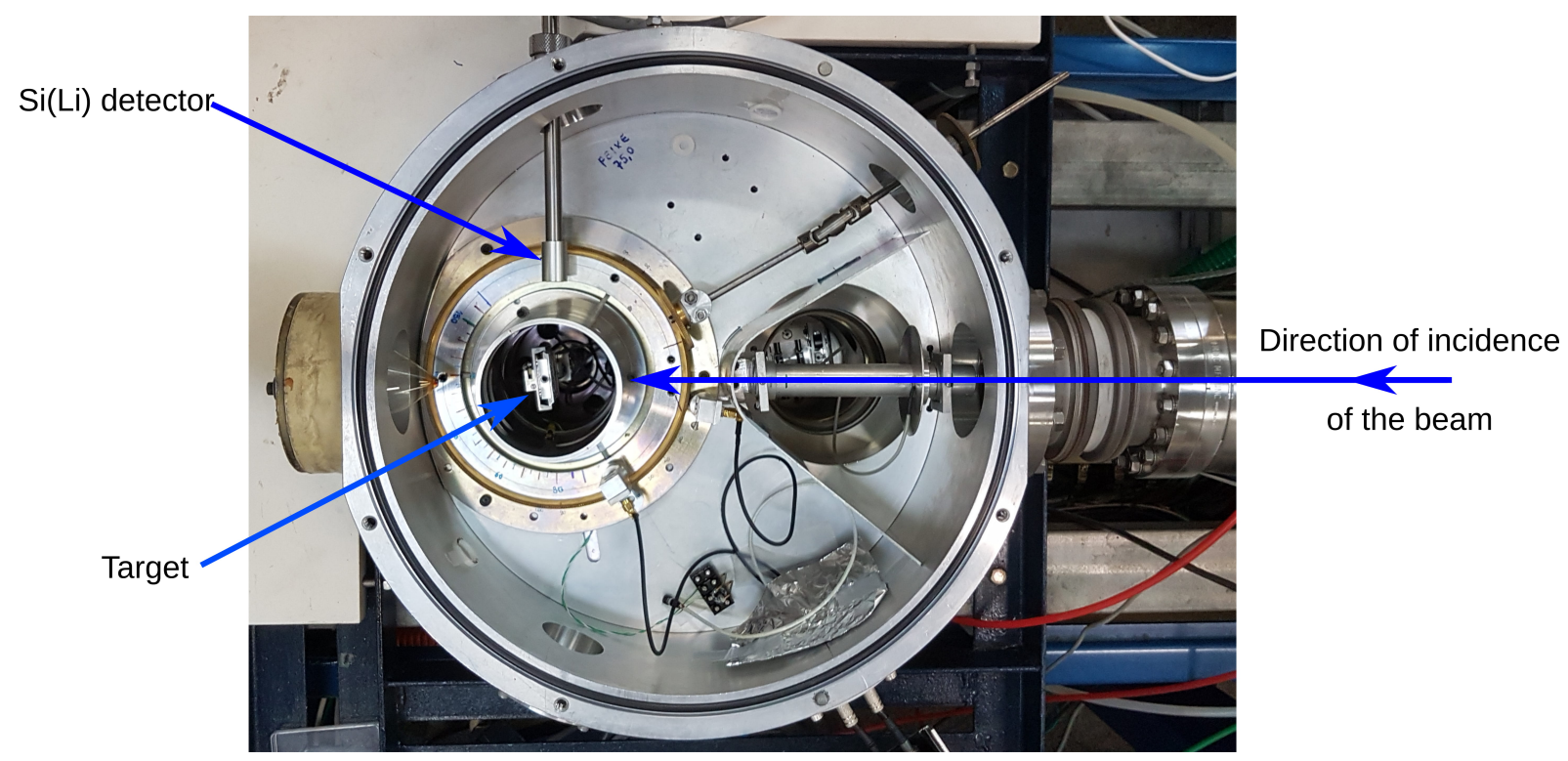

Figura 4.1: Chamber of the PIXE setup at LAMFI, USP.

Figura 4.2: Schematic representation of the experimental PIXE setup at LAMFI, USP.

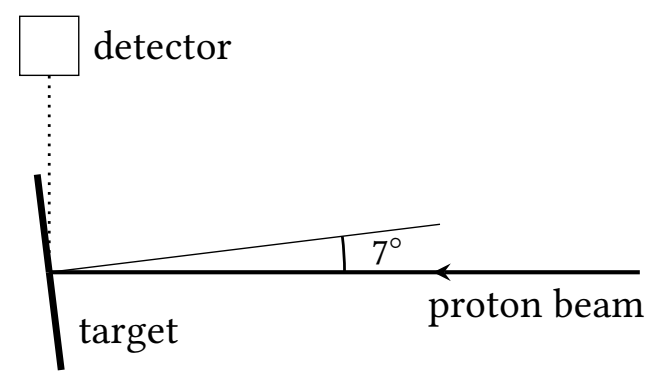

\subsection{Experimental setup}

Measurements of two PIXE spectra have been collected at LAMFI, Laboratório de Análise de Materiais e Feixes Iônicos, at the University of São Paulo. Figure 4.1 displays an upper view of the chamber of the PIXE setup at LAMFI. The support for the target can be seen, in this picture, near the center of the chamber, the detector is positioned on the side of the target, and, finally, the direction of incidence of the beam is indicated with an arrow. The energies of the incident beam of $\mathrm{H}^{+}$have been set to 2.0 and $3.0 \mathrm{MeV}$. The total deposited charge has been chosen as $20 \mu \mathrm{C}$. The angle of detection of the $\mathrm{x}$-rays relative to the beam is fixed at $90^{\circ}$, which is a construction characteristic of the chamber in use at LAMFI. Besides, the x-rays have been measured with a cylindrical $\mathrm{Si}(\mathrm{Li})$ detector with dimensions of $10 \mathrm{~mm}^{2} \times 150 \mu \mathrm{m}$.

The target consists of a self-supporting gold film, positioned with an angle of incidence of $7^{\circ}$, as represented in Figure 4.2. The thickness of the target has been measured at LAMFI with the Rutherford Back Scattering (RBS) technique. The value obtained is $26.34(14) \mu \mathrm{g} / \mathrm{cm}^{2}$, so the effective thickness of the material as seen by the impinging protons in the PIXE measu- 
rements, due to the angle of incidence, is obtained by dividing $26.34 \mu \mathrm{g} / \mathrm{cm}^{2}$ by $\cos 7^{\circ}$, giving $26.54 \mu \mathrm{g} / \mathrm{cm}^{2}$.

This target specimen has been kindly made available by LAMFI and has been employed because it suited rather well the development schedule of the present work. Unfortunately, it has not been specifically manufactured for an experiment intended to measure the bremsstrahlung effects on the PIXE spectrum. Some considerations, useful for selecting the thickness of the target in future measurements, are presented in Section 4.4.

Table 4.1 summarizes the main aspects of the experiment performed at LAMFI. The naming convention adopted in the laboratory has been kept. Finally, the resulting spectra are shown in Figure 4.3, for energies (a) 3 and (b) $2 \mathrm{MeV}$. The data files generated at LAMFI contain metadata in the first 25 lines, hence the gap at the very left of the spectra in Figure 4.3. For example, line 9 holds the total running time of the corresponding experiment.

Tabela 4.1: PIXE spectra obtained at LAMFI, on April 10, 2017.

\begin{tabular}{|c|c|c|c|c|c|}
\hline Spectrum & Proton Energy & Element & Detection angle & Deposited charge & Thickness \\
\cline { 1 - 4 } $\mathrm{R} 040094 \mathrm{X}$ & $3.0 \mathrm{MeV}$ & $\mathrm{Au}$ & $90^{\circ}$ & $20 \mu \mathrm{C}$ & $26.34(14) \mu \mathrm{g} / \mathrm{cm}^{2}$ \\
\cline { 1 - 2 } $\mathrm{R} 040097 \mathrm{X}$ & $2.0 \mathrm{MeV}$ & & & & \\
\hline
\end{tabular}

In order to compare the calculated DDCSs with the measured spectra, the detection effects inherent to the measurement must be taken into account. The following aspects of the experiment must be considered:

- self attenuation,

- detector response function $(F)$,

- detector efficiency $(\mathcal{E})$,

- solid angle of the detector $\left(\Omega_{d}\right)$,

- inverse energy width of the channel $(\mathrm{d} E)$,

- energy position of the channel $E$,

- number of incident protons $\left(N_{p}\right)$,

- thickness of the specimen $(t)$,

- pile-up effect.

The first step is to calculate the inverse energy width of the channels, $1 / \mathrm{d} E$, i.e. number of channels per unit of energy, which, in this work, is considered to be constant. In this case, $1 / \mathrm{d} E$ can be calculated by fitting a function of the form $C(x)=x / \mathrm{d} E+b$, where $x$ is the energy of the photon, $C(x)$ is the channel number as a function of the photon energy, and $b$ is a 
(a)



(b)

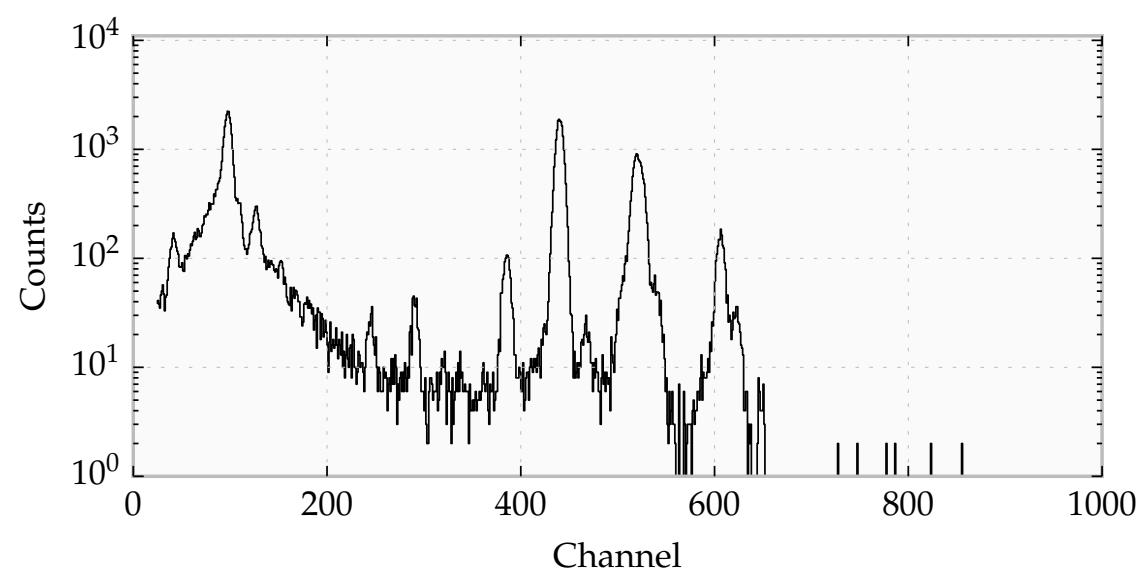

Figura 4.3: PIXE spectra (a) R040094X, obtained with a $3 \mathrm{MeV}$ proton beam, and (b) R040097X, with a $2 \mathrm{MeV}$ proton beam, for a gold target and a detection angle of $\theta=90^{\circ}$.

constant. By manually identifying a few emission lines in each spectrum, it is possible to fit the parameters $1 / \mathrm{d} E$ and $b$. Naturally, the most intense lines are the easiest to encounter and thus serve better this purpose.

For the gold target, the $\mathrm{L}$ and $\mathrm{M}$ series are visible and some of the most intense lines can be distinguished. In particular, the emission lines $\mathrm{M} \alpha_{1}, \mathrm{~L} \alpha_{1}, \mathrm{~L} \beta_{1}$, and $\mathrm{M} \gamma_{1}$ have energies $2.1216,9.713,11.4425$, and $13.3816 \mathrm{keV}$, respectively (Pia et al., 2009). They can be identified in the spectrum R040094X, for example, where the center of the corresponding peaks are located at channels 97, 440, 519, and 607, respectively, in Figure 4.3 a. Fitting the linear equation results in $\mathrm{d} E=0.022 \mathrm{keV} /$ channels and $b=-0.017$ channels. With this procedure, the spectra can be converted to photon energy. Figure 4.4 shows the result for spectrum R040094X. Three additional peaks can be spotted in Figure 4.4: they are produced by impurities in the target or, more probably, by the material of the chamber or the target holder hit by a stray beam. They 


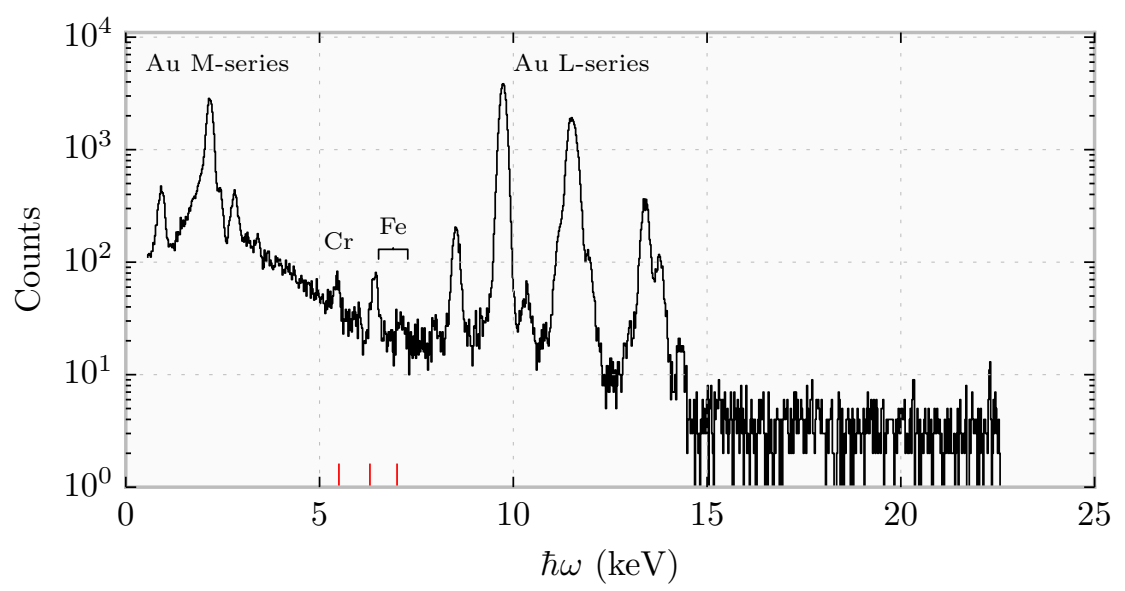

Figura 4.4: PIXE spectrum R040094X, obtained for a gold target with a $3 \mathrm{MeV}$ proton beam. In this case, the $\mathrm{L}$ and $\mathrm{M}$ series of characteristic $\mathrm{x}$-rays are visible. The peaks correspond to Fe and $\mathrm{Cr}$ (impurities) are also indicated.

correspond to the $\mathrm{K} \alpha$ and $\mathrm{K} \beta$ lines of iron and the $\mathrm{K} \alpha$ line of chrome, as indicated by the labels above them.

Additionally, the attenuation of the photons in the target material has been considered. By working the geometry involved in the positioning of the target relative to the proton beam and the detector, represented in Figure 4.2, it can be shown that a photon travels a maximum path inside the target material given by $t_{\max }=t / \sin \left(\theta_{\text {beam }}\right)$, where $\theta_{\text {beam }}$ is the angle between the direction of incidence of the proton beam and the direction perpendicular to the target surface. In the present case, this results in $t_{\max }=216.1 \mu \mathrm{g} / \mathrm{cm}^{2}$.

The exponential attenuation is given by $I / I_{0}=\exp \left[-(\mu / \rho) t_{\max }\right]$, where $\mu / \rho=\sigma_{\mathrm{pe}} / u A$ is the mass attenuation coefficient, with $u$, the atomic mass unit; $A$, the relative atomic mass of the target element; and $\sigma_{\mathrm{pe}}$, the cross section for the interaction of the photons with the medium. Here, $\sigma_{\mathrm{pe}}$ is the photoelectric absorption cross section, as discussed in Section 2.4.3, and it is plotted in Figure 2.17b for the case of gold. Finally, the transmission coefficient of the photons in the target material is represented in Figure 4.5, which is applied to the calculated cross sections in Sections 4.3 and 5.1.3. 


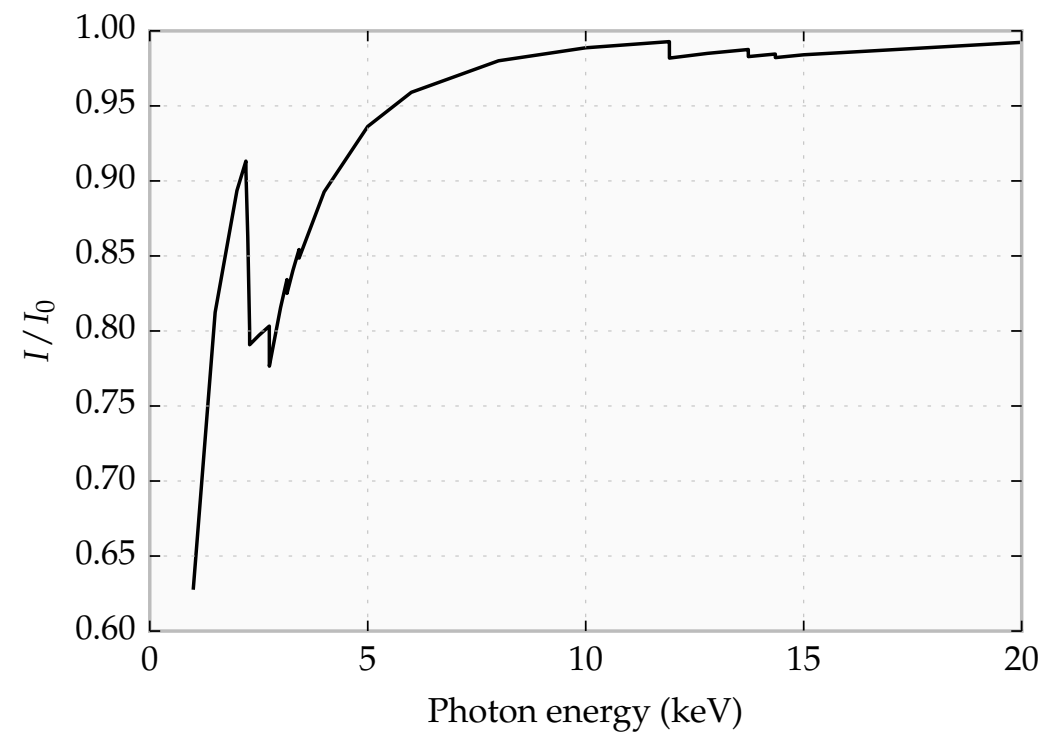

Figura 4.5: Transmission coefficient for the gold target, whose characteristics are given in Table 4.1, and the geometry of the PIXE setup employed at LAMFI. 


\subsection{Detection effects}

The detection effects considered in the present work are those associated to the response function, the efficiency, and the pile-up in the electronic chain.

\subsubsection{Detector response function}

In order to analyze the PIXE spectra obtained with $\mathrm{Si}(\mathrm{Li})$ detectors, it is crucial to know how the detector responds to a photon with a well defined energy. Indeed, the main feature of the recorded pulse-height distribution is a Gaussian peak, whose width is associated with the energy resolution of the spectrometry system. However, such Gaussian peak is not the only feature present, but typically a low energy tail is found as well. The resulting pulse height spectrum is actually called the response function of the detector.

The response function primarily reflects the different ways in which the radiation interacts with the detection medium, as discussed in Subsection 2.4.3, consequently it depends on the photon energy and the material of the sensitive volume. It is also a consequence of the inherent statistical fluctuations of the measured signal made up from discrete charges, besides the design and geometry of the detector. The response function for the $\mathrm{Si}(\mathrm{Li})$ detector can be essentially modeled by the formula (Reed and Ware, 1972)

$$
\begin{aligned}
& F\left(E, E_{0}\right)=\frac{1-k}{\sqrt{2 \pi \sigma^{2}}} \exp \left(-\frac{\left(E-E_{0}\right)^{2}}{2 \sigma^{2}}\right)+\frac{k}{\sqrt{2 \pi \sigma^{2}}} \exp \left(-\frac{\left(E-\left(E_{0}-E_{\mathrm{SiK} \alpha}\right)\right)^{2}}{2 \sigma^{2}}\right)+ \\
& k=\frac{0.035 \epsilon}{1-0.035 \epsilon} \quad, \\
& \epsilon=\left\{\begin{array}{l}
\text { erfc }\left(-\frac{\left(E-E_{0}\right)^{2}}{\sigma \sqrt{2}}\right), \\
0 \quad \text { if } \quad E_{0} \leq U_{\mathrm{SiK}} \quad, \\
\int_{0}^{\pi / 2}\left[\int_{0}^{d} \exp \left\{-\left(\mu_{S i K} \sec \theta+\mu_{S i}\right) x\right\} \mu_{S i} \mathrm{~d} x\right] \frac{1}{2} \sin \theta \mathrm{d} \theta .
\end{array}\right.
\end{aligned}
$$

Equation (4.2.1a) represents the detector response as a function of the recorded energy $E$ for an incident primary photon of energy $E_{0}$. Here, the shape of the peaks is assumed to be a Gaussian, where $\sigma$ is the standard deviation. Then, $\sigma$ is related to the full width at half 
maximum (FWHM) of the Gaussian curve by FWHM $=2.35 \sigma$. In general, if the FWHM is dominated by the contribution of the electronic chain, it can be assumed to be constant and equal to the detector resolution. For simplicity, the FWHM is considered to be constant in the present work.

In Equation (4.2.1a), the first Gaussian term is the full energy peak, while the second term corresponds to the escape peak (see below), where $E_{\mathrm{Si} \alpha}=1.739 \mathrm{keV}$ is the energy of the $\mathrm{K} \alpha$ line for silicon. The third term, modeled by an error function, represents the incomplete charge collection in the detector volume. The multiplicative constant $c$ must be estimated from the measured spectra.

In general, the Compton scattering contributes to the response function as a continuous distribution below the full energy peak, since the secondary electron ejected from the atom has a continuous spectrum. However, in the present case, the cross section for the Compton scattering is much smaller than that of the photoelectric absorption and only affects rather low recorded energies (as discussed in Section 2.4.3, see Figure 2.17). Therefore, the Compton scattering is not included in the response function adopted here, Equation (4.2.1a).

The escape peak can occur when the ionization of a silicon atom causes the emission of an x-ray from the filling of a vacancy in the K-shell of Si. This photon can escape the detection volume, resulting in a small peak in the response function at an energy $E_{0}-E_{\mathrm{SiK} \alpha}$. In the present case, only the $\mathrm{K} \alpha$ line is considered, because the intensity of the silicon $\mathrm{K} \beta$ line is less than $2 \%$ of that of the former (Reed and Ware, 1972).

The normalization of the Gaussian terms in Equation (4.2.1a) is given by the intensity ratio $k$, Equation (4.2.1b), between the escape peak and the full energy peak. In turn, $k$ takes into account the proportion of $\mathrm{K}$-shell ionizations that emit silicon $\mathrm{K} \alpha$ photons relative to the total number of ionizations produced in the detector, by the factor 0.035 (Reed and Ware, 1972). Moreover, $\epsilon$, appearing in Equation (4.2.1b), denotes the fraction of silicon $\mathrm{K} \alpha \mathrm{x}$-rays that can escape from the detector and it is given by Equation (4.2.1c) (Reed and Ware, 1972). The escape peak is produced only by incident photons with an energy $E_{0}$ greater than the silicon $\mathrm{K}$ absorption edge, $U_{\mathrm{Si} K}=1.839 \mathrm{keV}$. Therefore, the response function of the $\mathrm{Si}(\mathrm{Li})$ detector consists of the full energy Gaussian peak at $E_{0}$, if $E_{0} \leq U_{\mathrm{Si} \mathrm{K}}$, and the full energy peak at $E_{0}$ plus the escape peak at $E_{0}-E_{\mathrm{SiK} \alpha}$, if $E_{0}>U_{\mathrm{SiK}}$. The flat continuum, corresponding to the incomplete charge collection and described by the term with the error function, is always present.

The fraction $\epsilon$ is obtained from geometric considerations, as illustrated in Figure 4.6, leading to Equation (4.2.1c), where $x$ is the distance of the ionized silicon atom from the face of the detector and $\theta$ is the angle in which the characteristic x-ray has been emitted relative to the direction perpendicular to the face of the detector. Besides, $\mu_{S i K}$ is the linear attenuation 


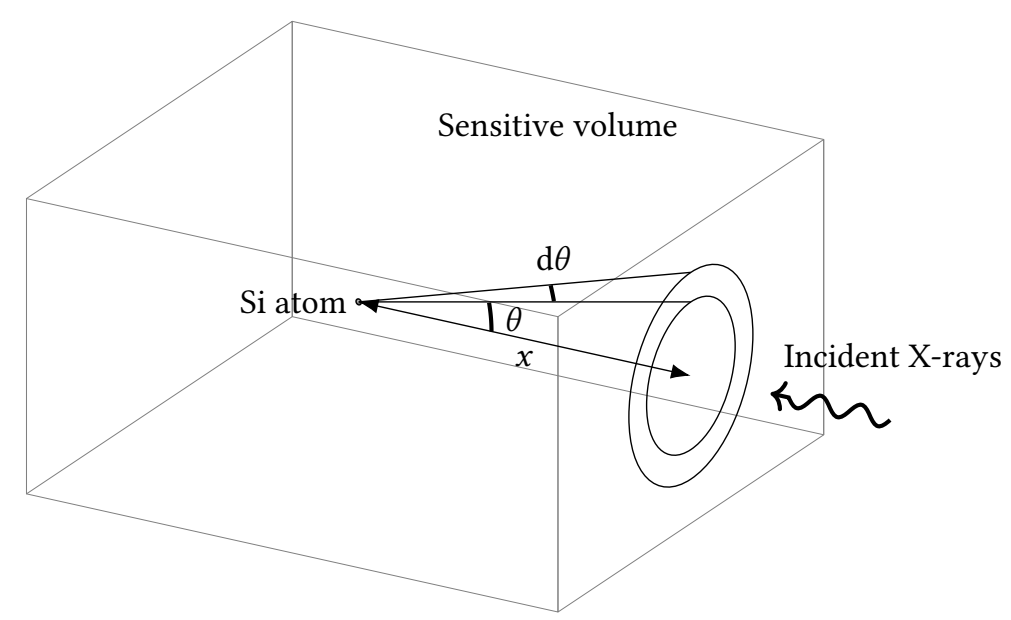

Figura 4.6: Diagram of the geometrical for the calculation of the parameter $\epsilon$ given by Equation $(4.2 .1 \mathrm{c})$.

coefficient for the silicon $\mathrm{K} \alpha$ x-rays $(1.739 \mathrm{keV}), \mu_{S i}$ is the linear attenuation coefficient for the energy of the incident photon and $d$ is the thickness of the sensitive volume of the detector.

Figure 4.7 illustrates an example of the response function for $E_{0}=4 \mathrm{keV}$. The curve in black corresponds to Equation (4.2.1a), while the colored ones represent each individual term of the equation.

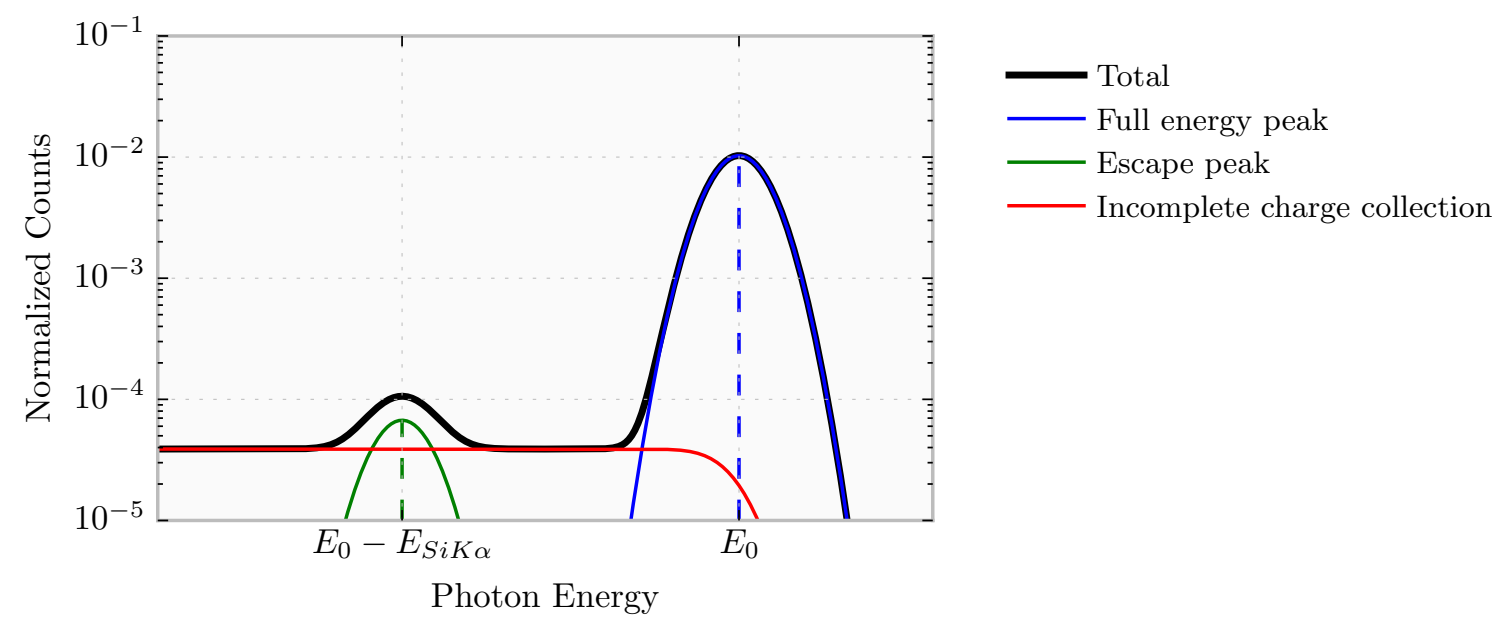

Figura 4.7: Example of the response function of the $\mathrm{Si}(\mathrm{Li})$ detector. Each contribution to Equation (4.2.1a) is represented individually (coloured lines), along with their sum being the total response function (black line). The blue line represents the full energy peak (at $E_{0}=4 \mathrm{keV}$ ) and the green line is the escape peak (at $E_{0}-E_{\mathrm{SiK} \alpha}$ ), besides the flat continuum due to incomplete charge collection, drawn in red.

In the present work, knowledge of the response function of the $\mathrm{Si}(\mathrm{Li})$ detector used at 
LAMFI is essential for the comparison between theoretical and experimental bremsstrahlung spectra. The theoretical DDCS for proton bremsstrahlung, i.e. the sum of the contributions from the five processes presented in Section 2.3, or the x-ray production cross sections discussed in Section 2.4 are calculated and a convolution of the resulting spectrum with the response function of the detector is performed. Denoting, for example, the DDCS for proton bremsstrahlung by $\mathrm{d}^{2} \sigma / \mathrm{d} \Omega \mathrm{d}(\hbar \omega)$, the convolution with the response function $F$ of the detector is given by

$$
\left(\frac{\mathrm{d}^{2} \sigma}{\mathrm{d} \Omega \mathrm{d}(\hbar \omega)} * F\right)(E)=\int_{0}^{E_{\mathrm{p}}} \frac{\mathrm{d}^{2} \sigma}{\mathrm{d} \Omega \mathrm{d}(\hbar \omega)}(\varepsilon) F(E, \varepsilon) \mathrm{d} \varepsilon
$$

where the symbol $*$ denotes, as usual, the convolution of two functions, $E$ is the recorded energy (given by the measured pulse-height applying the calibration), $\varepsilon$ is the energy of the primary photon impinging on the detector, and $E_{\mathrm{p}}$ is the energy of the proton. The determination of the parameters defining the response function from Equation (4.2.1a) is discussed in Section 4.3.

\subsubsection{Detector efficiency}

When a charged particle enters the sensitive volume of a detector, it immediately excites and ionizes the medium, rapidly forming ion pairs in its path thus producing a pulse signal. In this case, every incident particle entering the detector can be, in principle, recorded as a pulse. The detector thus has a counting efficiency of $100 \%$.

On the other hand, when an x-ray photon enters the sensitive volume of a $\mathrm{Si}(\mathrm{Li})$ detector, other processes (i.e. photoelectric absorption or Compton scattering, see Section 2.4.3) must take place in the medium in order to produce secondary charged particles that in turn generate electron-hole pairs thus resulting in a signal. Because the sensitive volume of the detector has a finite dimension (hundreds of micrometers to few millimeters), the x-ray photon may escape without interactions. In this case, the number of recorded pulses is less than the number of photons that enters the sensitive volume of the detector. Therefore, the detector has a counting efficiency less than $100 \%$.

This counting efficiency is called the intrinsic efficiency of the detector, $\varepsilon_{\mathrm{i}}$, and it is a function of the energy of the incident radiation. The intrinsic efficiency also depends on the thickness and the material of the sensitive volume. In addition, the total efficiency of a semiconductor detector must take into account the presence of layers of material external to the sensitive volume. They cause the absorption of the incident photons and are accounted as correction factors to the efficiency.

In the case of a $\mathrm{Si}(\mathrm{Li})$ detector, a correction factor for the attenuation due to a beryllium window on the external face of the detector, $f_{\mathrm{Be}}$, and a thin gold contact on the face of the detector, $f_{\mathrm{Au}}$, are considered. Therefore, the efficiency $\mathcal{E}$ of the $\mathrm{Si}(\mathrm{Li})$ detector, as a function of 
the photon energy $E$ is given by (Hansen et al., 1973)

$$
\mathcal{E}=\varepsilon_{\mathrm{i}} f_{\mathrm{Be}} f_{\mathrm{Au}}
$$

The intrinsic efficiency in Equation (4.2.3), for a sensitive volume with depth $d$, is given by

$$
\varepsilon_{\mathrm{i}}=1-\exp \left(-\mu_{S i} d\right)
$$

where $\mu_{S i}$ is the linear attenuation coefficient for silicon. The correction factors $f_{\mathrm{Au}}$ and $f_{\mathrm{Be}}$ are obtained by the formula

$$
f_{j}=\exp \left(-\mu_{j} x_{j}\right)
$$

where $\mu_{j}$ and $x_{j}$ are the linear attenuation coefficient and the thickness of the layer of element $j$, respectively.

Figure 4.8 illustrates the contributions of the intrinsic efficiency and the correction factors to the total efficiency of the detector as given by Equations (4.2.3)-(4.2.5). The values for the depth of the sensitive volume of the detector and the thickness of the beryllium and gold layers have been obtained as discussed in Section 4.3, being applicable to the detector installed at LAMFI USP. The values of the respective attenuation coefficients have been extracted from the NIST database ${ }^{1}$.

In this figure, the dashed red curve represents the intrinsic efficiency, which starts at a value of 1 , for low energy photons, and rapidly decreases for energies above $\approx 10 \mathrm{keV}$. The dependence of $\varepsilon_{\mathrm{i}}$ on the thickness of the sensitive volume of the detector, see Equation (4.2.4), shows that larger dimensions yield more interactions between the incident radiation and the medium, increasing the intrinsic efficiency for higher photon energies. The blue and yellow dashed curves are obtained from Equation (4.2.5) with $\mu_{B e}$ and $\mu_{A u}$ the linear attenuation coefficient of beryllium and gold, respectively. The graph shows a considerable absorption of low energy photons by the beryllium window, which has a more significant effect when compared to the gold contact deposited on the detector face.

Finally, the resulting detector efficiency (black solid line), calculated from Equation (4.2.3), shows that the detector is suitable for photon energies above $\sim 1.5 \mathrm{keV}$ and has considerable attenuation effects for energies above $\sim 10 \mathrm{keV}$. The detector efficiency is, therefore, one important aspect to be taken into account in the comparison of theory with data. In particular, it excludes any sensitivity of the measurements to the QFEB contribution.

\footnotetext{
${ }^{1}$ https://www.nist.gov/pml/x-ray-mass-attenuation-coefficients. Accessed on March 2020.
} 


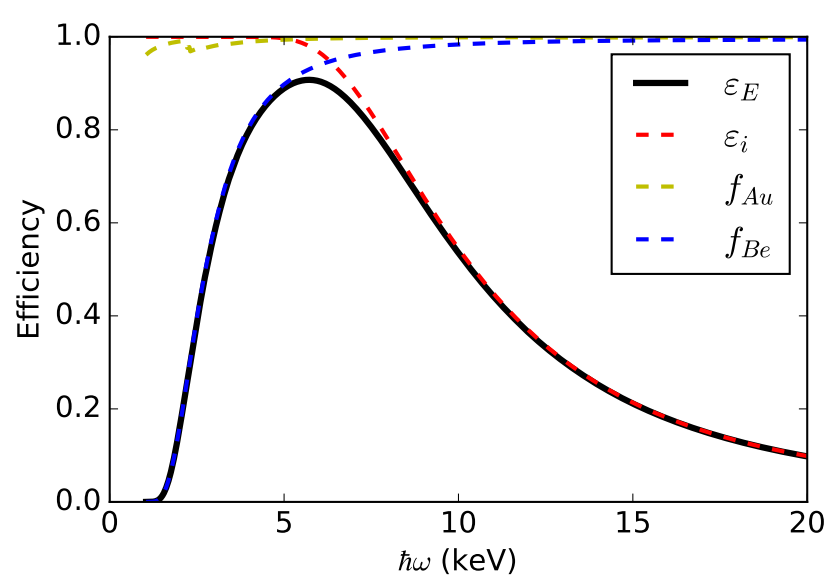

Figura 4.8: Detector efficiency (red dashed line) and correction factors for the attenuation due to the beryllium window (blue dashed line) and the gold contact on the face of the detector (yellow dashed line). The total efficiency is represented by the black solid line.

\subsubsection{Pile-up effect}

The x-ray photons produced by the impact of protons arrive at the detector at randomly spaced time intervals. It is thus possible that two consecutive photons enter the sensor in a short time interval and are recorded as one single count. As a result, the two initial events are not separated and only one count is recorded, causing a distortion in the final spectrum. An event, in this case, is the interaction (photoelectric absorption) of a photon with the detector. A count, on the other hand, is the final electronic signal actually registered by the spectroscopy system.

This effect is called pile-up and it is very sensitive to the characteristics of the electronics employed. The pulse received by the amplifier from the first signal has an exponential tail characterized by a time $\tau$ of order of $\mu$ s (Leo, 1994). When the consecutive signal arrives within a time less than $\tau$, the previous pulse is increased. Because the amplitude of the pulses is proportional to the energy, the resulting information on the photon energy is distorted.

Usually, an electronic circuit called pile-up rejector (PUR) is employed to reduce the pile-up effect in PIXE spectroscopy. This method essentially enables the system to discard a pulse that is expected to be distorted by the pile-up effect of two close events (Johansson et al., 1995). Moreover, there are different methods to calculate the pile-up effect. In this sense, the most accurate methods apply Monte Carlo simulations of both the detection and electronic signal formation processes. Nonetheless, Monte Carlo simulations still tend to cost too much computation time, besides requiring many details about the detection system (Barradas and Reis, 2006).

Therefore, in order to calculate the pile-up effect in PIXE spectra, the analytical algo- 
rithm of Wielopolski and Gardner (Gardner and Wielopolski, 1977; Wielopolski and Gardner, 1976, 1977), as described by Barradas and Reis (2006), has been employed in the present work. This method is based on a statistical analysis and requires few experimental parameters, such as the shaping time of the amplifier and the total pulse rate of the experiment.

In this approach, the pile-up of two consecutive events, which would have amplitudes $A_{i}$ and $A_{j}$ individually recorded by the detector, causes the system to register only one count with amplitude $A_{k}$. This resulting pulse is called the sum peak. In the final PIXE spectrum, the pile-up of the two initial pulses means that they are not accounted in their corresponding channels, $i$ and $j$, but in channel $k$ as one single pulse. The amplitude $A_{k}$ depends on the initial amplitudes $A_{i}$ and $A_{j}$ and the time separation between those events. In fact, $A_{k}$ can have any value in the interval $\max \left(A_{i}, A_{j}\right) \leq A_{k} \leq A_{i}+A_{j}$ (Barradas and Reis, 2006; Wielopolski and Gardner, 1976). Moreover, the amplitude $A_{i}$ of the signal is linearly related to the channel $i$, that is

$$
A_{i}=a i+b
$$

where $a$ and $b$ are constants depending on the electronics of the system. Therefore, in the following equations, the amplitudes can be expressed in terms of the corresponding channels.

As usual, the Poisson distribution gives the probability $P_{n}$ of observing $n$ events in a time interval $t$

$$
P_{n}=\frac{\mu^{n}}{n !} e^{-\mu}
$$

where $\mu=N_{t} t$ is the event rate, that is, the average number of events observed during time $t$, with $N_{t}$ the total pulse rate of the experiment. The probability for the pile-up of events from channels $i$ and $j$ forming the count in channel $k$, denoted by $P_{i j k}$, is obtained by combining three probabilities (Barradas and Reis, 2006; Wielopolski and Gardner, 1976), all derived from the Poisson distribution. First, the probability that no pulses occur between pulses $i$ and $j$, where $t^{\prime}$ is the time between them. This is obtained from Equation (4.2.7) with $n=0$ and $\mu=N_{t} t^{\prime}$

$$
P_{1}=\left(N_{t} t^{\prime}\right)^{0} \exp \left(-N_{t} t^{\prime}\right) / 0 !=\exp \left(-N_{t} t^{\prime}\right)
$$

Second, the probability that the pulse $j$ occurs between $t^{\prime}$ and $t^{\prime}+\mathrm{d} t^{\prime}$, which is obtained with $n=1$ and $\mu=N_{t} \mathrm{~d} t^{\prime}$

$$
P_{2}=\left(N_{t} \mathrm{~d} t^{\prime}\right)^{1} \exp \left(-N_{t} \mathrm{~d} t^{\prime}\right) / 1 ! \approx N_{t} \mathrm{~d} t^{\prime}
$$




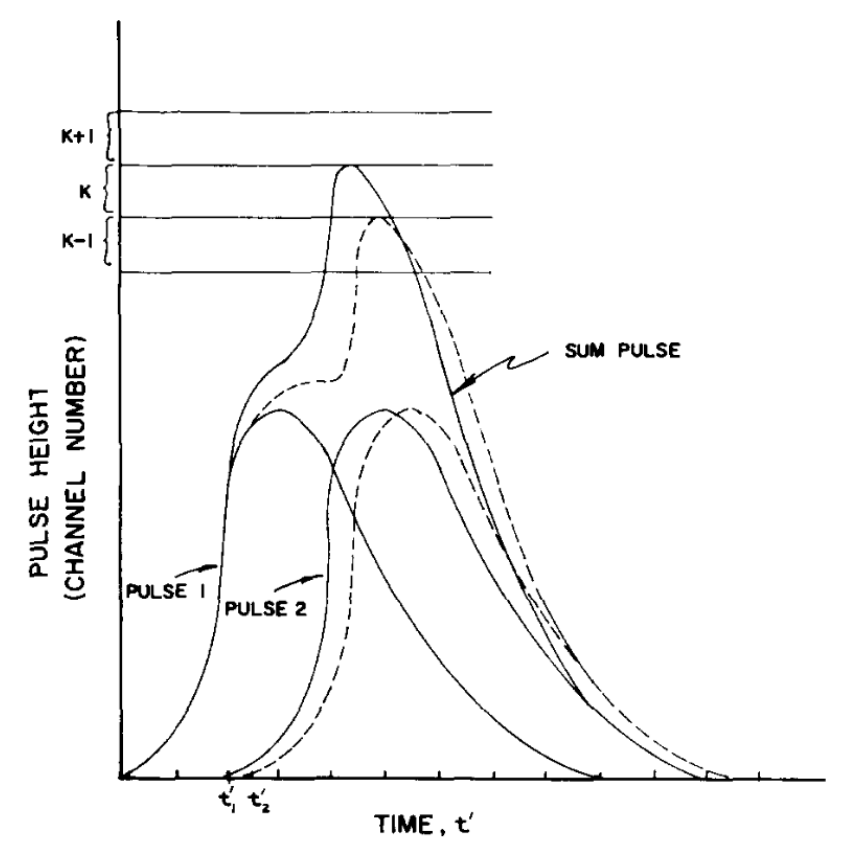

Figura 4.9: Pile-up of two pulses. Source: Wielopolski and Gardner (1976).

Lastly, the probability that there are zero pulses between $t^{\prime}+\mathrm{d} t^{\prime}$ and the time of the end of the first pulse, $T_{\text {shape }}$. In this case, the expression is obtained with $n=0$ and $\mu=N_{t}\left(T_{\text {shape }}-t^{\prime}\right)$

$$
\begin{aligned}
P_{3} & =\left(N_{t}\left(T_{\text {shape }}-t^{\prime}\right)\right)^{0} \exp \left(-N_{t}\left(T_{\text {shape }}-t^{\prime}\right)\right) / 0 ! \\
& =\exp \left(-N_{t}\left(T_{\text {shape }}-t^{\prime}\right)\right) .
\end{aligned}
$$

Finally, the probability $P_{i j k}$ is found by multiplying $P_{1}, P_{2}$, and $P_{3}$, which is a differential probability per unit time, and integrating it over the time period that results in a pulse pile-up with amplitude $k$. This time period, defining the interval of integration, spans from $t_{1}^{\prime}(i, j, k)$ to $t_{2}^{\prime}(i, j, k-1)$, and it can be better understood by analyzing Figure 4.9, from Wielopolski and Gardner (1976). In this graph, the first pulse starts at $0 ; t_{1}^{\prime}$ and $t_{2}^{\prime}$ are the times at which the second pulse must start so that the sum peak falls at the upper and lower borders of channel $k$, respectively. That is, if the second pulse starts at a time earlier than $t_{1}^{\prime}$, the sum peak will have amplitude $A_{k+1}$ or greater, and if it starts at a time later than $t_{2}^{\prime}$, the sum peak will be registered with amplitude $A_{k-1}$ or smaller.

The probability $P_{i j k}$ is thus given by

$$
\begin{aligned}
P_{i j k} & =\int_{t_{1}^{\prime}(i, j, k)}^{t_{2}^{\prime}(i, j, k-1)}\left(\mathrm{d} P_{i j k} / \mathrm{d} t^{\prime}\right) \mathrm{d} t^{\prime} \\
& =N_{t} \exp \left(-N_{t} T_{\text {shape }}\right) \Delta t_{i j k},
\end{aligned}
$$


with

$$
\begin{aligned}
& \Delta t_{i j k}=t^{\prime}(i, j, k-1)-t^{\prime}(i, j, k), \\
& t^{\prime}(i, j, k)=T_{\text {rise }}\left[\frac{(i+j-k)(i+j)}{i j}\right]^{1 / 2},
\end{aligned}
$$

where $T_{\text {shape }}$ is the shaping time depending on the amplifier, and $T_{\text {rise }}$ is the time the pulse takes to reach its maximum value. Equation (4.2.12) has been obtained by Wielopolski and Gardner (1976) approximating the pulse shape with a parabola. The channel $k$, where the resulting pulse is recorded, is expected to be in the interval $\max (i, j) \leq k \leq i+j$.

Therefore, with Equation (4.2.11), the lost events $L_{i j k, i}$ and $L_{i j k, j}$ from channels $i$ and $j$, respectively, and the gained counts $G_{i j k, k}$ in channel $k$ are given by (Barradas and Reis, 2006; Wielopolski and Gardner, 1976)

$$
L_{i j k, i}=L_{i j k, j}=G_{i j k, k}=N_{t} P_{i} P_{j} P_{i j k}
$$

In the case of a calculated theoretical spectrum without pile-up, $P_{i}$ and $P_{j}$ correspond to the yield in channels $i$ and $j$ divided by the integral yield. Finally, Equations (4.2.11)-(4.2.13) allow to calculate the lost and gained counts in each channel of a theoretical spectrum.

In this work, the procedure described above is applied to a calculated bremsstrahlung spectrum, as discussed in Chapter 5, in order to compare the theoretical models for proton bremsstrahlung with the experimental data obtained in laboratory at LAMFI USP. The parameters needed for the pile-up calculation are described in Section 5.2.

\subsection{Determination of the parameters of the response func- tion from experiment}

This section describes the method employed to estimate the parameters involved in the calculations of the detector response function and efficiency. Although the spectrum R040094X is taken as the reference case, the parameters obtained here are valid for the spectrum R040097X as well, since both have been measured sequentially under the same general conditions.

The detection efficiency is given by Equation (4.2.3). This formula considers the intrinsic efficiency of the sensitive volume and the attenuation factors due to the beryllium window and the gold contact on the face of the detector. The linear attenuation coefficients for beryllium, silicon, and gold necessary to calculate the detector efficiency have been obtained from the NIST database. Moreover, the geometry and material composition of the detector 
are also crucial for the detection efficiency. Table 4.2 lists the nominal values for the main dimensions of the $\mathrm{Si}(\mathrm{Li})$ detector used at LAMFI.

The response function of the detector is given by Equation (4.2.1). Normally, the response function is determined by calibrating the detector with a source of monoenergetic radiation. With this procedure, the resolution, $\sigma$, and the incomplete charge collection, $c$, parameters can be accurately determined. In the present case, however, the detector resolution has been set to the constant value stated by the manufacturer of $150 \mathrm{eV}$, and the incomplete charge collection has been estimated as follows.

Firstly, the characteristic peaks in one of the PIXE spectra are identified, see Section 2.4. Because the DDCS for bremsstrahlung tends to decrease with the photon energy, it is preferable to focus on higher energy peaks, such as those from the L-series, thus reducing the effects from bremsstrahlung in the spectrum. In the present work, the database by Pia et al. (2009) has been used to provide the energies of the characteristic x-rays.

The intensity of the emission lines must be calculated using Equations (2.4.1)-(2.4.3e), and the self attenuation depicted in Figure 4.5 is applied to the cross section. The value of the parameter $c$ (see Equation (4.2.1)) is adjusted so that the convolution of the characteristic yields with the response function produces Gaussian peaks as similar as possible to the experimental ones. The result is shown in Figure 4.10, representing the convolution of the cross section of the emission lines with the response function of the detector taking into account the self attenuation (in orange) and not including it (in green), for comparison. The value of the parameter $c$ thereby established is $c=4.0 \times 10^{-3}$.

When the distance $r$ between the detector and the target is much greater than the characteristic dimension of the face of the detector, the solid angle can be approximated by $\Omega_{d}=A / r^{2}$, where $A$ is the area of the detector (Knoll, 2000). In the case of the device available at LAMFI, the nominal values are $A=10 \mathrm{~mm}^{2}$ and $r=70 \mathrm{~mm}$ (see Table 4.2).

Lastly, the number of incident protons in the sample is simply calculated by the total deposited charge, measured in the experiment, divided by the elementary charge.

By taking into account the aspects of the experiment mentioned above with the parameters estimated here, the bremsstrahlung yield, i.e. the counting of photons per channel

Tabela 4.2: Geometry of the $\mathrm{Si}(\mathrm{Li})$ detector used at LAMFI.

\begin{tabular}{|l|c|c|}
\hline thickness of the sensitive volume & $d$ & $150 \mu \mathrm{m}$ \\
\hline thickness of the beryllium window & $x_{B e}$ & $100 \mu \mathrm{m}$ \\
\hline thickness of the gold contact & $x_{A u}$ & $50 \mathrm{~nm}$ \\
\hline area & $A$ & $10 \mathrm{~mm}^{2}$ \\
\hline distance from the source & $r$ & $70 \mathrm{~mm}$ \\
\hline
\end{tabular}




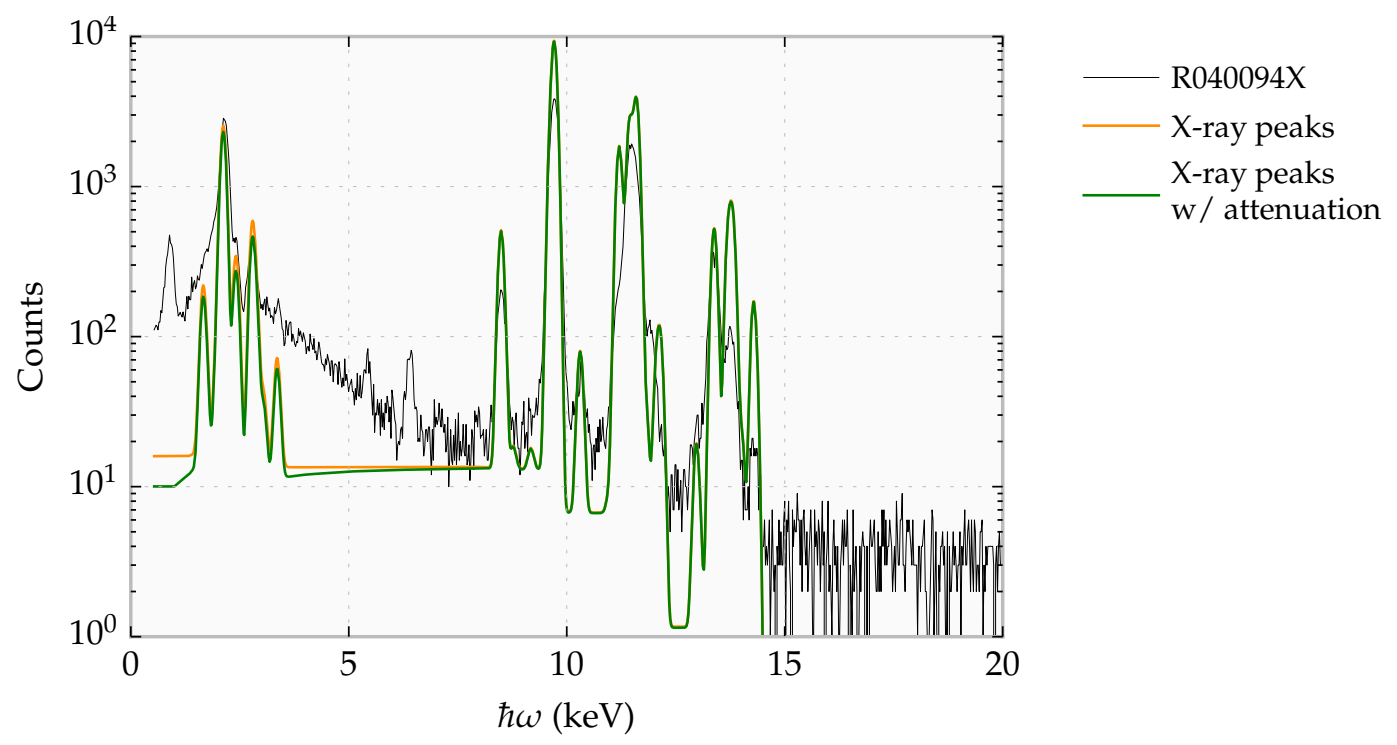

Figura 4.10: PIXE spectrum R040094X. The orange line represents the convolution of the characteristic $\mathrm{x}$-ray lines with the response function of the detector. The green line takes into account the self attenuation in the target.

as a function of the photon energy, $Y(E)$, can be calculated as

$$
Y(E)=\mathrm{d} E N_{p} t \frac{\Omega_{d}}{4 \pi}\left(\frac{\mathrm{d}^{2} \sigma}{\mathrm{d}(\hbar \omega) \mathrm{d} \Omega} e^{-\mu(\hbar \omega) t_{\max }} \mathcal{E}(\hbar \omega) * F\right)(E)
$$

where $\mu$ is the linear attenuation coefficient of the target for photons of energy $\hbar \omega$ and $t_{\max }$ is the thickness of the target seen by a photon emitted in the direction of the detector, see Section 4.1 .

\subsection{Estimation of the thickness of the target for future PIXE experiments}

An important contribution to the bremsstrahlung spectrum induced by protons with energies of few $\mathrm{MeV}$ comes from the SEB process. In this process, as discussed in Section 2.3.1, secondary electrons ejected by the impact of the proton travel through the material while losing energy in inelastic collisions, subsequently producing bremsstrahlung. Nonetheless, if the ejected electron escapes the material before producing bremsstrahlung, the contribution from the SEB process will be underestimated. Therefore, it is important that the target is thick enough to stop most of the ejected electrons, whilst not causing the impinging protons to lose too much energy (to allow a direct comparison with a calculation where a well defined value is assumed). 
Further experiments might be undertaken in the future in order to investigate the effects of the bremsstrahlung in PIXE spectra. In this case, the thickness of the target employed must be calculated according to the range of the secondary electrons, so that they do not escape from the medium at a too early stage. It is thus necessary to calculate the maximum energy of the ejected electrons for a given energy of the proton. The maximum energy that can be transferred from the impinging proton to an electron of the target atom considered at rest, $T_{\max }$, is given in the BEA by Equation (2.3.10). However, as noted in Section 2.3.1.1, because of the VD in the BEA model, the energy distribution of the ejected electrons present non zero values above $T_{\max }$. Hence, in order to estimate the maximum energy of the secondary electrons and define the thickness of the target, the following steps are considered.

1. Calculate the energy distribution, up to $100 \mathrm{keV}$, of the ejected electrons for $\theta_{\mathrm{e}}=10^{\circ}, 45^{\circ}$, and $90^{\circ}$, and $E_{\mathrm{p}}=1$ and $4 \mathrm{MeV}$, where $\theta_{\mathrm{e}}$ is the ejection angle of the electron and $E_{\mathrm{p}}$ is the energy of the proton;

2. Calculate the integral of the energy distribution of the ejected electrons up to $99 \%$ of the total area and take the corresponding upper limit of the integral as the maximum energy of the ejected electrons, $E_{\mathrm{e}}^{\max }$;

3. Calculate the range for the electrons with energy $E_{\mathrm{e}}^{\max }$, in the continuous slowing down approximation (CSDA);

4. Calculate the energy loss of the proton for the corresponding $E_{\mathrm{p}}$ in the thickness corresponding to the CSDA range of the electrons found in the previous step.

In the first step, the energy distribution of the electrons is calculated using Equation (2.3.12), in the BEA with the hydrogenic VD. The maximum transferable energies with $E_{\mathrm{p}}=1$ and $4 \mathrm{MeV}$ are $T_{\max }=2.2$ and $8.7 \mathrm{keV}$, respectively. Therefore, the upper limit of $100 \mathrm{keV}$ in the calculations of the energy distribution of the electrons is a safe value for the purpose of the next steps. Moreover, the calculations have been performed for three values of $\theta_{\mathrm{e}}$ in order to check the energy distribution for each case. They are shown in Figure 4.11 for a gold target and energies of the proton of (a) 1 and (b) $4 \mathrm{MeV}$. Smaller ejection angles represent tighter constraints on the thickness of the target.

As a second step, the distributions shown in Figure 4.11 are integrated. With that, the upper limits of the integrals which correspond to $99 \%$ of the total area of each curve can be found. Figure 4.12 shows the energy distributions for $\theta_{\mathrm{e}}=10^{\circ}$ up to $20 \mathrm{keV}$. The hatched areas correspond to $99 \%$ of the total integral of the spectrum. The values thus obtained are $E_{\mathrm{e}}^{\max }=2.4$ and $9.8 \mathrm{keV}$, for $E_{\mathrm{p}}=1$ and $4 \mathrm{MeV}$, respectively. It can be noted that these values are close to the corresponding maximum transferable energies $T_{\max }$. 
(a)

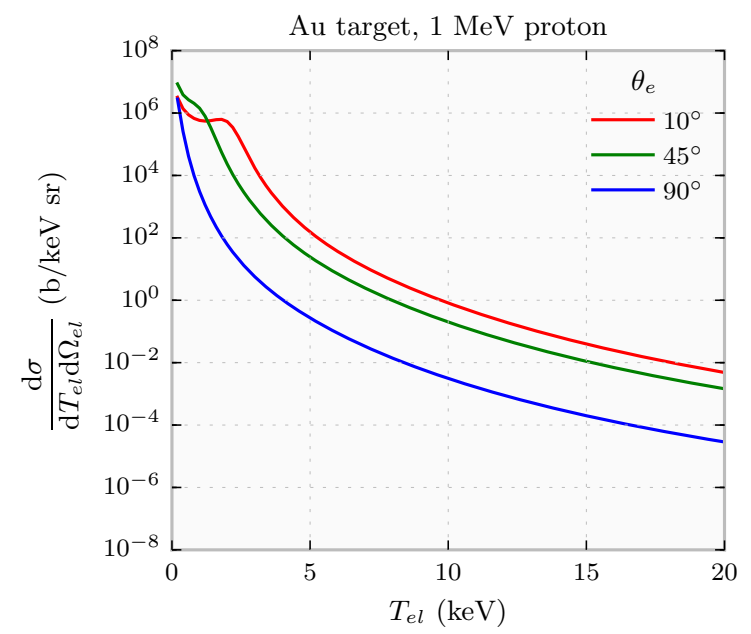

(b)

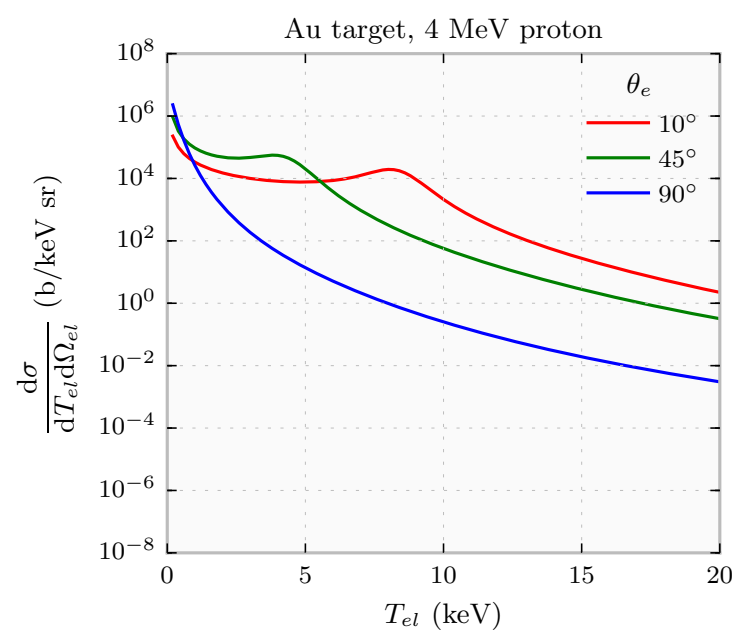

Figura 4.11: Energy distribution of the ejected electrons. Calculations were performed up to $100 \mathrm{keV}$, although the graphs show only the part up to $20 \mathrm{keV}$.

(a)

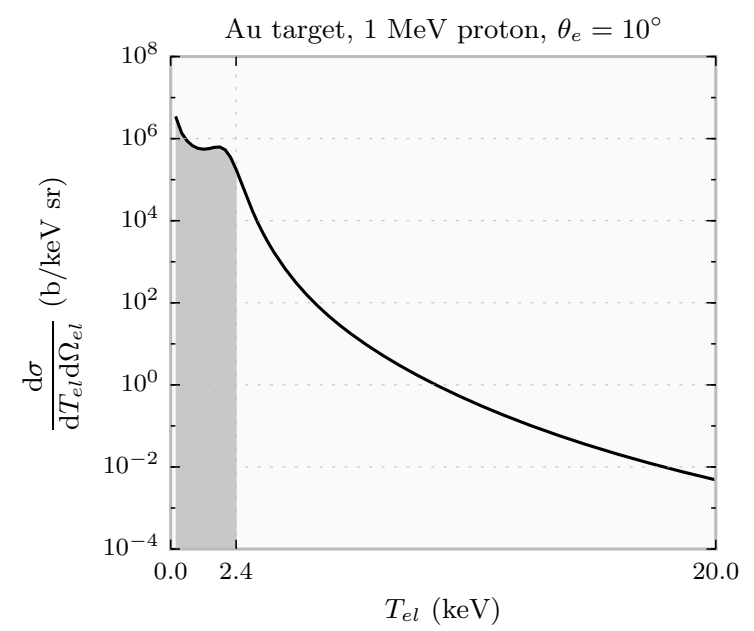

(b)

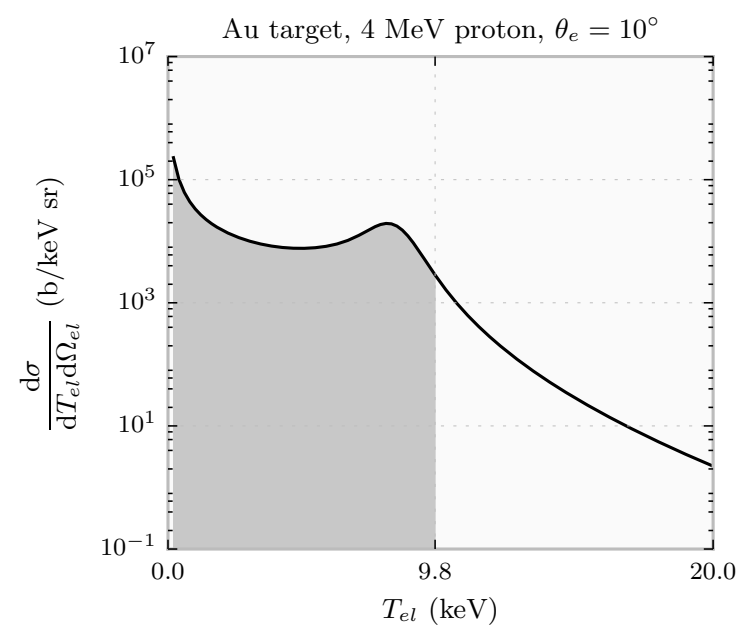

Figura 4.12: The hatched areas correspond to $99 \%$ of the integral of the total spectrum, which has been calculated up to an energy of the ejected electrons of $100 \mathrm{keV}$.

Finally, the CSDA range for the secondary electrons can be calculated for $E_{\mathrm{e}}^{\max }$ using the NIST ESTAR database ${ }^{2}$. In the case of $E_{\mathrm{e}}^{\max }=9.8 \mathrm{keV}$, the value obtained for the thickness of the material is around $790 \mu \mathrm{g} / \mathrm{cm}^{2}$.

Additionally, it is possible to calculate the energy loss of the proton in the target, considering the thickness obtained from the analysis discussed above. In this case, the energy

\footnotetext{
${ }^{2}$ https://physics.nist.gov/PhysRefData/Star/Text/ESTAR.html. Accessed on April 2020.
} 
loss has been calculated employing the NIST PSTAR database ${ }^{3}$. When considering $E_{\mathrm{p}}=1 \mathrm{MeV}$ and the angle of incidence equal to $7^{\circ}$, the energy loss of the proton in $790 \mu \mathrm{g} / \mathrm{cm}^{2}$ of gold is around $5.1 \%$.

Therefore, it can be noted that the thickness of the target used in the experiment partially allows for the ejected electrons to escape the material before producing bremsstrahlung. That can lead to an overestimated value of the calculated SEB contribution, mainly for higher energies of the photons. It must be noted that bremsstrahlung is mostly produced at the beginning of the slowing down of the secondary electrons, ionization of the medium being more and more important for lower and lower energy electrons. On the other hand, in the present case, the energy loss of the impinging proton can be fully neglected.

\section{Summary}

Measurements of the PIXE spectra have been performed at LAMFI, USP, to be compared in the next Chapter 5 with the models of the proton bremsstrahlung processes implemented in the program described in Chapter 3. The target consists of a self-supporting gold film, with a thickness of $26.34 \mu \mathrm{g} / \mathrm{cm}^{2}$. The energies of the incident proton beam have been set to 2 and $3 \mathrm{MeV}$. The angle of detection of the x-rays is fixed by the setup at $90^{\circ}$.

However, the PIXE spectrum is the result of the characteristic $x$-rays and the bremsstrahlung emission taking into account self-absorption in the target, the efficiency of the detector, as well as the convoluted with its response function, and the pile-up effect inherent to the measurement system. Therefore, knowledge of the characteristics of the detector and some aspects of the electronics of the system are important in order to perform a comparison between the theoretical DDCS for bremsstrahlung and the experimental spectrum. In this chapter, the self-attenuation, the efficiency, the response function of the detector, and the pile-up effect are discussed, so that these aspects of the measurements can be included in the calculated spectrum.

PIXE spectroscopy typically relies on $\mathrm{Si}(\mathrm{Li})$ detectors, which present good detection efficiency and energy resolution. The response signal recorded by the detector when submitted to monoenergetic radiation is called the response function of the detector. In this case, the width of the response peak represents the energy resolution of the detector. The $\mathrm{Si}(\mathrm{Li})$ employed at LAMFI present an energy resolution of about $150 \mathrm{eV}$ (FWHM), for $5.9 \mathrm{keV}$ photons, a typical value.

The intrinsic efficiency of the detector is a function of the energy of the radiation and it depends on the thickness and the material of the sensitive volume. In addition, the

\footnotetext{
${ }^{3}$ https://physics.nist.gov/PhysRefData/Star/Text/PSTAR.html. Accessed on April 2020.
} 
total efficiency of the detector must take into account the attenuation due to layers of passive materials deposited on the front of the active part of the device. Generally, the detector efficiency decreases for energies outside the interval $\sim 5-30 \mathrm{keV}$, as is also the present case.

The pile-up effect occurs when two consecutive photons enter the detector in a sufficiently short interval of time, causing the system to record one single count with distorted amplitude. The method for pile-up calculation reviewed here is based on a statistical analysis and has the advantage of requiring only a few experimental parameters describing the electronics of the spectrometry system.

The parameters of the response function, which has to be convoluted with the expected emission lines from the L-series of gold, have been adjusted so that the measured spectra are well reproduced in a region where the continuous contribution from proton bremsstrahlung is negligible. The cross section of the characteristic $\mathrm{x}$-rays is calculated according to the equations described in Section 2.4 .

At last, as a remark, a method is described to estimate the optimal thickness of the target in future PIXE experiments aimed at better analyzing the SEB process. These calculations are necessary because the secondary electrons ejected by the proton can escape the target medium before producing bremsstrahlung, in which case the theoretical SEB DDCS would overestimate the experiment. Moreover, the thickness of the target must take into account the energy lost by the impinging proton and the attenuation of the emitted photons in the material. 



\section{Capítulo 5}

\section{Results}

The ionization DDCS in the BEA is a function of the velocity of the electrons and therefore it is sensitive to the model of the VD. As a consequence, the SEB and AB+RI DDCSs are also affected by the VDs of the electrons. Before the present work, calculations of the SEB and $\mathrm{AB}+\mathrm{RI} \mathrm{DDCSs}$ have only been performed using the analytical expression of the hydrogenic $1 \mathrm{~s}$ VD. Moreover, the same expression is generally applied to all subshells of the target atom.

In the present work, the influence of the VD on the ionization cross section and the $\mathrm{SEB}$ and $\mathrm{AB}+\mathrm{RI} \mathrm{DDCSs}$ is investigated. For this purpose, the analytical hydrogenic VD is replaced by numerical calculations of the DHFS VDs. With this approach, relativistic effects on the VD for the inner shells and the screening of the Coulomb field in the outer shells of the target atom can be accounted for. Additionally, in this Chapter, the hydrogenic 1s VD is replaced by the analytical expressions for the hydrogenic VD of the K, L, and M (sub)shells.

Calculations have been performed for aluminum, silver, and gold targets, with proton energies of 1 and $4 \mathrm{MeV}$, using the hydrogenic and the DHFS VDs for both the ionization and the SEB and $\mathrm{AB}+\mathrm{RI}$ DDCSs. The total cross sections for ionization within the BEA are compared to the tabulated values obtained from the PWBA and the ECPSSR theories. In this way, the ionization DDCS with DHFS VDs can be studied before being implemented in the calculations of the SEB and AB+RI DDCSs. Moreover, the results are presented for individual shells separately, thus allowing for a study of the effects of the VD in each shell of the target element.

Lastly, the calculations of the bremsstrahlung DDCS using the DHFS VDs are compared with the PIXE spectra obtained in laboratory. The experiments have been performed for a gold target and proton energies of 2 and $3 \mathrm{MeV}$. The predicted spectrum is the sum of the proton bremsstrahlung DDCSs discussed in this work. Comparisons between the experimental spectra and the theoretical DDCSs are undertaken after including the experimental effects.

In this Chapter, Section 5.1 shows the comparisons between the results using the 
hydrogenic and the DHFS VDs, for the ionization DDCSs and total cross sections (Subsection 5.1.1), the SEB DDCSs (Subsection 5.1.2), and the AB+RI DDCSs (Subsection 5.1.3). Finally, the comparisons between the calculated DDCSs and the experimental PIXE spectra are shown in Section 5.2, taking into account the detection effects discussed in Section 4.2.

\subsection{Calculations with DHFS VDs}

The hydrogenic VDs have been obtained under two assumptions. The first and most straightforward option corresponds to the hydrogenic analytical expressions for the K, L, and $\mathrm{M}$ (sub)shells, according to Equation (2.3.16). In the case of the $\mathrm{N}$ subshells, the hydrogenic 1s wave functions is applied, as the analytical wave functions become very complex. Nonetheless, this method is somewhat different from that used in the previously published works, where the hydrogenic 1s VD was applied to all subshells. The second more realistic possibility is to employ the DHFS VDs, obtained from the radial wave functions of the DHFS method, see Equations (2.3.24). In particular, those adopted for the present work have been calculated numerically by J. M. Fernández-Varea, using the DHFS code developed at the University of Barcelona (Salvat and Fernández-Varea, 2019).

The results can be compared to the tabulated values from the PWBA and ECPSSR theories. The former is the plane-wave Born approximation and the latter is the most complete theory available to date that adds corrections for the energy loss (E), Coulomb deflection (C), binding-polarization through a perturbed-stationary-state approach (PSS), and relativistic effects (R) in the former. However, the chosen database by Pia et al. (2009) only contains total cross sections. Therefore total ionization cross section in the BEA have to be calculated numerically using Equation (3.1.1).

Moreover, the calculations of the SEB, $\mathrm{AB}+\mathrm{RI}$, and ionization DDCSs must be performed for each subshell of the target element. To accomplish this, the program developed to perform the numerical calculations, described in Chapter 3, receives the input parameters defining the target element, the energy of the proton, and the subshell identification of the target to select accordingly the binding energy, the occupation number, and the VD of the electrons. The following plot, however, for reasons of space, show the results for each shell, which are the sum of the contributions from every subshell with the correct occupation number (a different color is assigned to each shell).

The ionization cross sections are shown in Subsection 5.1.1 and the calculations of the SEB and AB+RI DDCSs are presented in Subsection 5.1.2 and 5.1.3, respectively. In all cases, the dashed curves represent the results from the calculations with the analytical expressions of the hydrogenic VDs, and the solid curves denote the results obtained with the numerical DHFS 
VDs.

The results presented in Subsection 5.1.1 and Subsection 5.1.2 have already been published (Ferro et al., 2020).

\subsubsection{Ionization cross sections}

Figure 5.1 shows the total ionization cross sections for each shell of the aluminum, silver, and gold target atoms as a function of the proton energy. The results in the BEA are compared to the reference values calculated with the PWBA (circles) and ECPSSR (triangles) theories, obtained from the mentioned database by Pia et al. (2009), for the K, L, and M shells. As expected, the outer shells, that have higher occupation numbers, have also higher ionization cross sections (and therefore contribute more to the SEB and $A B+R I$ processes). Note that the values have not been normalized to the occupation number of each shell, as is done in several of the published works.

As it can be seen in Figure 5.1, the cross sections calculated with the DHFS VDs are higher than the results obtained with the hydrogenic VDs in the region of higher energies of the proton. Furthermore, this behavior tends to invert for lower energies of the proton. The proton energy for which this inversion occurs, i.e. the crossing point of the two lines, depends on the element and shell. It must be noted that the crossing point can be located outside of the proton energy range shown in Figure 5.1.

Similar results have been obtained by Mukoyama (2015, 2017, 2018), who calculated the ionization cross sections in the BEA using VDs from hydrogenic, non-relativistic RoothaanHartree-Fock, and Dirac-Hartree-Slater wave functions, specifically for for the $\mathrm{M}_{5}$ subshell of gold (Mukoyama, 2015), the $\mathrm{M}_{5}$ subshell of bismuth (Mukoyama, 2017), and the $\mathrm{N}_{1}$ to $\mathrm{N}_{7}$ subshells of bismuth (Mukoyama, 2018). Therefore, the present results agree with the effect observed by Mukoyama and show that it remains valid for inner shells and when all shells are summed.

In general, the cross sections obtained within the BEA agree well with the PWBA and ECPSSR values. The results from the DHFS VDs tend to better match the PWBA ones for the $\mathrm{L}$ and $\mathrm{M}$ shells. A strong reduction of the cross sections for inner shells and low proton energies is present in the ECPSSR formalism, but not in the others, due to a better account of the binding effects. 

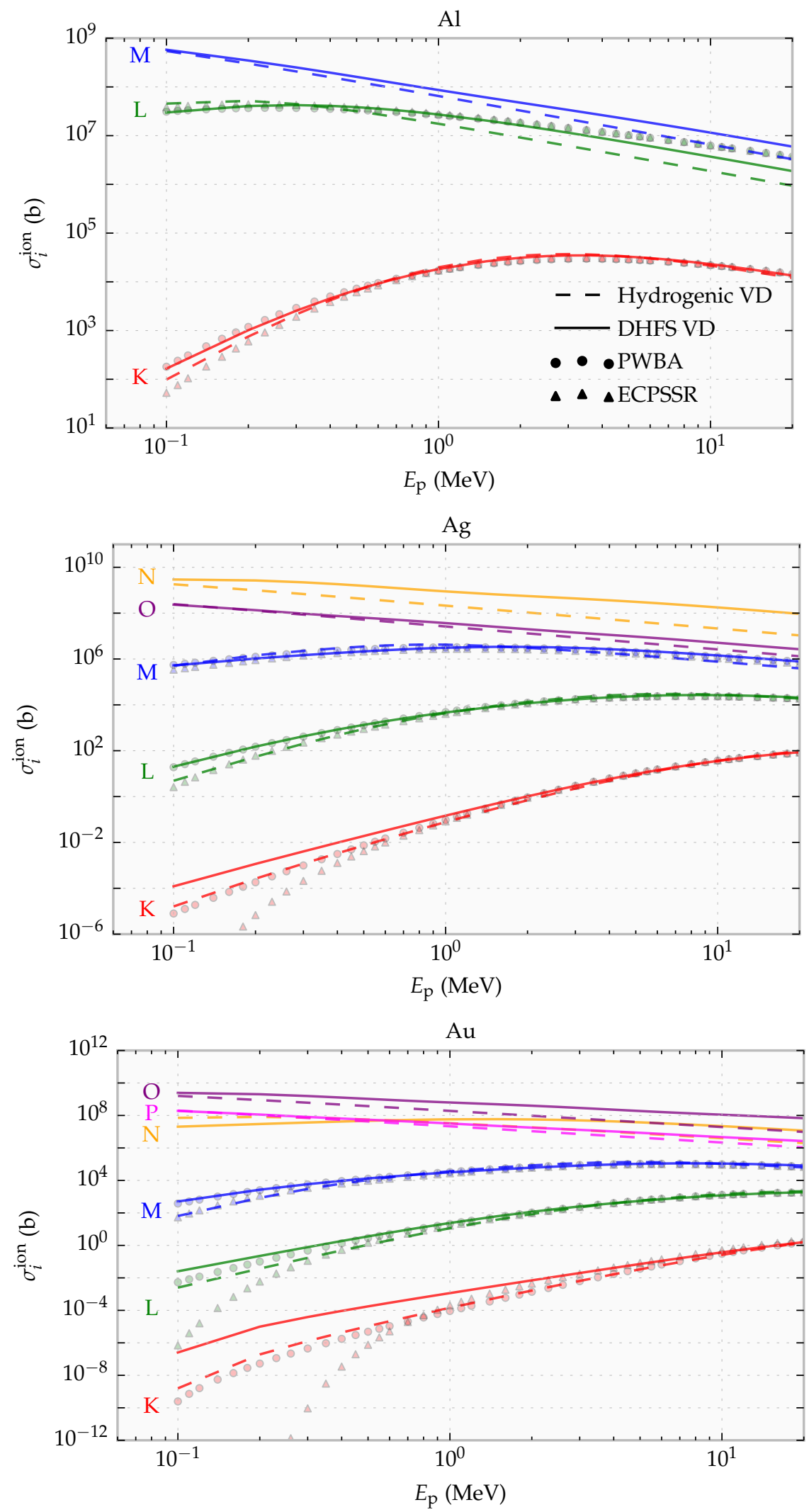

Figura 5.1: Ionization cross sections for $\mathrm{Al}, \mathrm{Ag}$, and $\mathrm{Au}$. Each line is the sum over the corresponding (sub)shells. The dashed and solid lines correspond to the BEA with hydrogenic and DHFS VDs, respectively. The circles and triangles are, respectively, PWBA and ECPSSR cross sections from the database by Pia et al. (2009). 


\subsubsection{SEB DDCSs}

The SEB DDCSs have been calculated using Equation (3.1.5), which has been introduced in the present work and employs a formulation in terms of the exponential integral function. In Chapter 3, Equation (3.1.5) has been validated with published references (Ishii et al., 1976; Yamadera et al., 1981). The comparison is performed for an aluminum target and proton energies of 1 and $4 \mathrm{MeV}$ (see Figure 3.8), using the VD from the analytical expression of the hydrogenic $1 \mathrm{~s}$ wave function, following the quoted references.

Here, following the discussion of the ionization cross sections in the previous section, the SEB DDCSs have been calculated for aluminum, silver, and gold targets; proton energies of 1 and $4 \mathrm{MeV}$; and a photon emission angle of $90^{\circ}$. As mentioned above, the calculations must be performed for each subshell with the corresponding VD. The results are first presented separately for each shell, i.e. by summing over the subshells, to analyze in detail of the effect of the VD model. Figures 5.2 and 5.3 show the plots for 1 and $4 \mathrm{MeV}$ proton energies, respectively. Finally, the total SEB DDCSs, i.e. summing the contributions from all electrons, are shown in Figure 5.4. In this way, it is possible to appreciate the overall influence of the VD on the SEB process.

Figures 5.2 and 5.3 clearly indicate that the outer shells contribute more to the SEB process. This result is consistent with the same effect observed for the ionization cross sections in Figure 5.1. Moreover, ionization cross sections based on the DHFS VDs are generally larger than those based on the hydrogenic ones especially for outer shells, see again Figure 5.1: the same effect is visible for the SEB DDCSs based on DHFS VDs.

To help the discussion of the impact of the VDs, the plots of Figures 5.2 and 5.3 also display $T_{\max }$, the maximum energy that the proton can transfer to an electron initially at rest (see Equation (2.3.10)), with a vertical line. The values are $T_{\max }=2.2 \mathrm{keV}$ and $T_{\max }=8.7 \mathrm{keV}$, for $E_{\mathrm{p}}=1 \mathrm{MeV}$ and $E_{\mathrm{p}}=4 \mathrm{MeV}$, respectively. As noted in Chapter 2, $T_{\max }$ reflects, as well, the maximum energy of the bremsstrahlung photon that the secondary electron can emit after being ejected by the proton. In this simple picture, the SEB DDCS should decrease sharply to zero at energies above $T_{\max }$. Since the electrons are not initially at rest, the inclusion of VDs leads to a smoothing of this transition. The approximation of an electron initially at rest is better for outer shells, as the speeds of the electrons are smaller, or higher proton energies. Indeed in such cases, Figures 5.2 and 5.3 exhibit sharper transitions.

Concerning the differences of the SEB DDCSs calculated with the DHFS and hydrogenic VDs, the former are generally larger than the latter for $\hbar \omega \gg T_{\max }$ because the DHFS VDs have typically stronger high momentum tails (see Section 3.1.2). On the other hand, the curves tend to coincide for $\hbar \omega<T_{\max }$ and outer shells, where the importance of the VD decreases as explained above. 

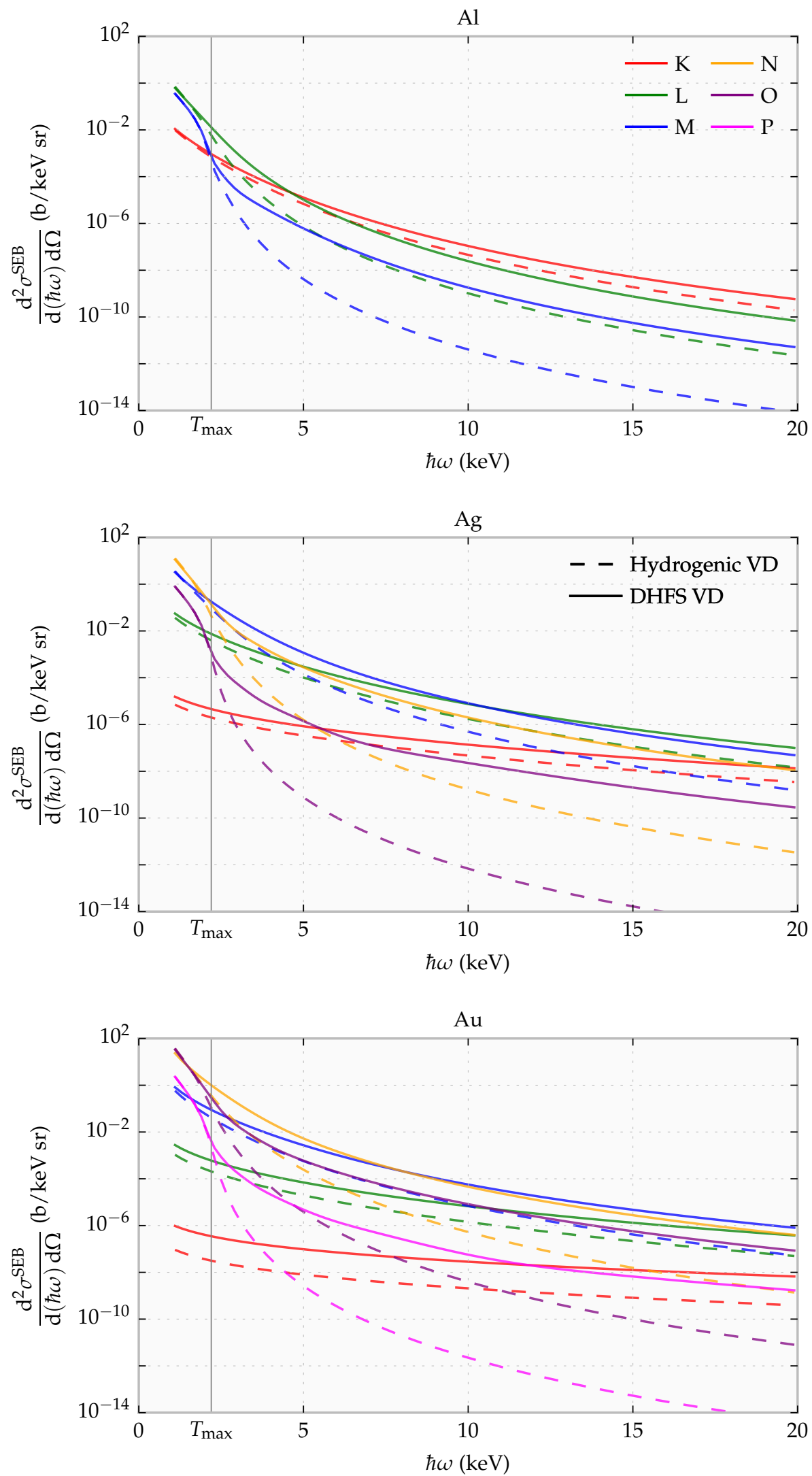

Figura 5.2: SEB DDCSs for protons impinging on $\mathrm{Al}, \mathrm{Ag}$, and $\mathrm{Au}$ with a kinetic energy of $1 \mathrm{MeV}$ radiating photons with an emission angle of $90^{\circ}$. Each line is the sum over the corresponding (sub)shells. Calculations employing hydrogenic VDs (dashed lines) and DHFS VDs (solid lines) are shown. 

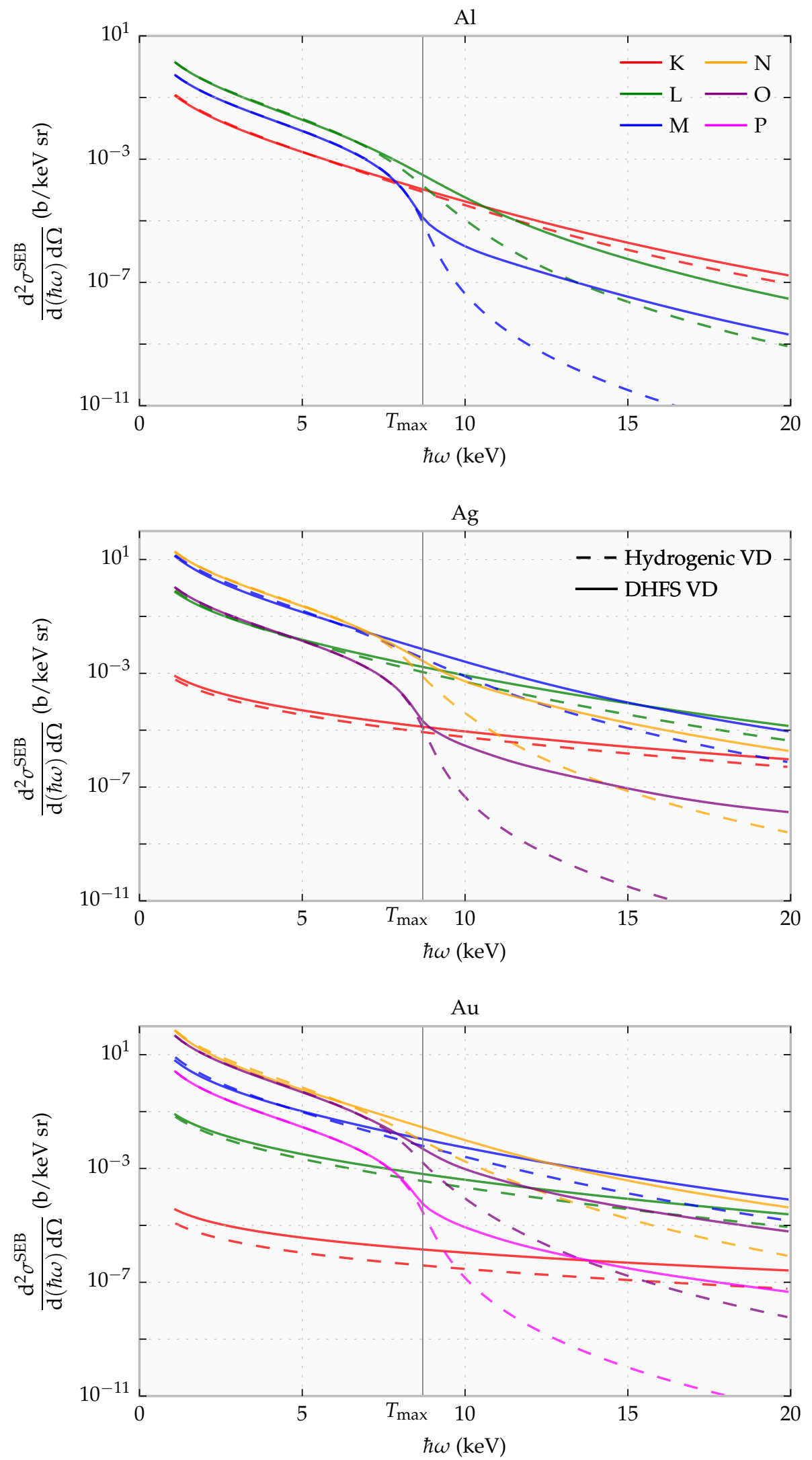

Figura 5.3: SEB DDCSs for protons impinging on $\mathrm{Al}, \mathrm{Ag}$, and $\mathrm{Au}$ with a kinetic energy of $4 \mathrm{MeV}$ radiating photons with an emission angle of $90^{\circ}$. Each line is the sum over the corresponding (sub)shells. Calculations employing hydrogenic VDs (dashed lines) and DHFS VDs (solid lines) are shown. 

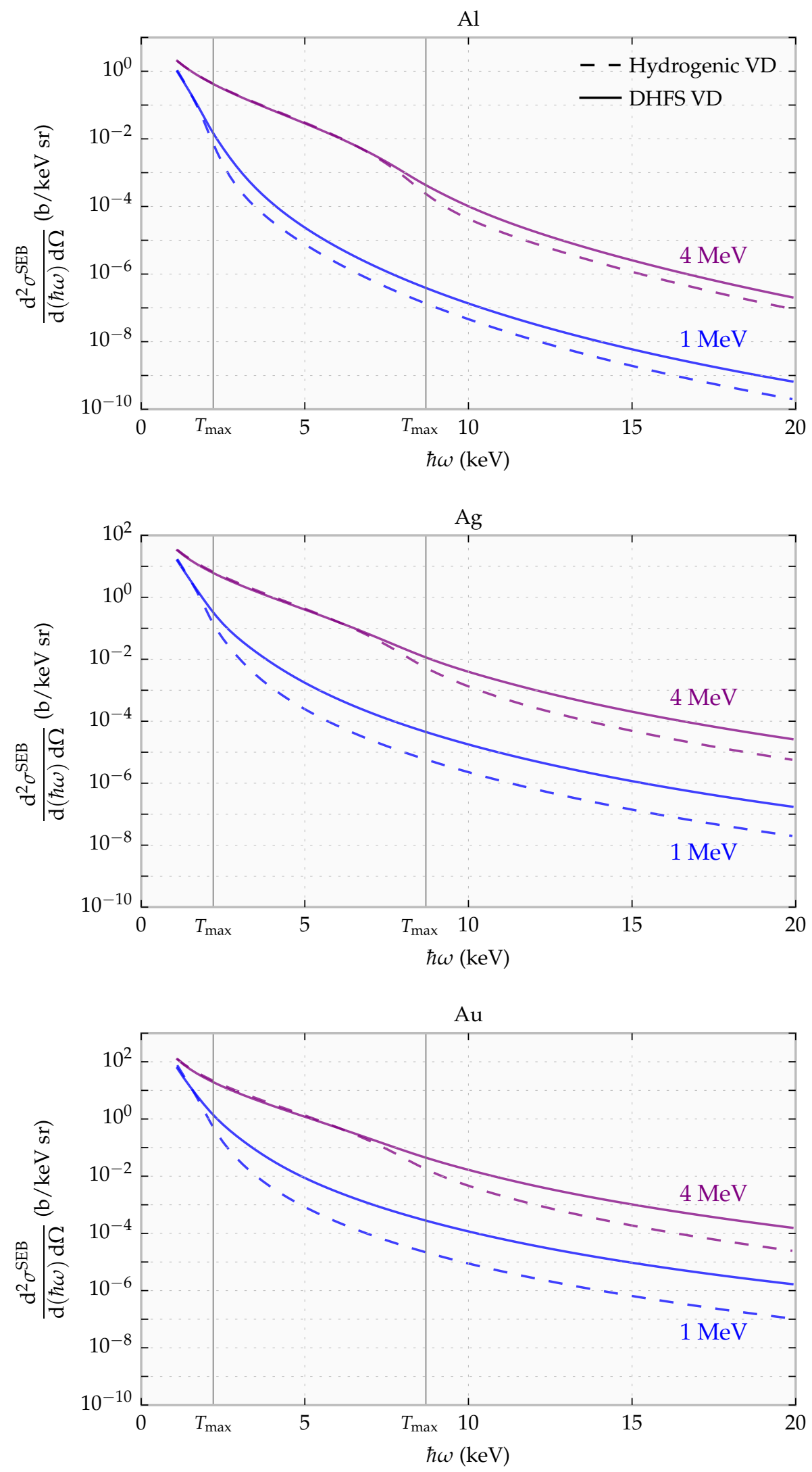

Figura 5.4: SEB DDCSs for protons impinging on $\mathrm{Al}, \mathrm{Ag}$, and $\mathrm{Au}$ with kinetic energies of $1 \mathrm{MeV}$ and $4 \mathrm{MeV}$ radiating photons with an emission angle of $90^{\circ}$. Calculations employing hydrogenic VDs (dashed lines) and DHFS VDs (solid lines) are shown. 
Finally, the influence of the VDs in the SEB DDCSs is also evident in Figure 5.4, where the contributions from all shells are summed. As before, the SEB DDCSs with hydrogenic and DHFS VDs coincide when $\hbar \omega<T_{\max }$, but the ones with the DHFS VDs are larger when $\hbar \omega \gg T_{\max }$.

\subsubsection{AB+RI DDCSs}

The $\mathrm{AB}+\mathrm{RI} \mathrm{DDCS}$ s have been calculated using Equations (2.3.38)-(2.3.40). Following the cases already discussed of the ionization cross sections and SEB DDCSs, the AB+RI DDCSs are presented for aluminum, silver, and gold targets; proton energies of 1 and $4 \mathrm{MeV}$; and a photon emission angle of $90^{\circ}$. Again, the subshells are calculated separately with the appropriate VDs, then the results are summed for each shell to reduce the number of curves to be plotted. Figures 5.5 and 5.6 display separately each shell for 1 and $4 \mathrm{MeV}$ proton energies, respectively, while the total AB+RI DDCSs are shown in Figure 5.7.

Similarly to Figures 5.2 and 5.3, the vertical line denotes $T_{\max }$ (see Equation (2.3.10)), the maximum energy that the proton can transfer to an electron initially at rest (and correspondingly the maximum energy of the photon that the electron can emit). In general, the transition around $T_{\max }$ is much sharper for AB+RI DDCSs than for SEB DDCSs (compare Figures 5.2, 5.3, and 5.4 with Figures 5.5, 5.6, and 5.7). The difference between inner and outer shells, where the approximation of an electron initially at rest works better, is also more pronounced than in the case of SEB DDCSs.

Outer shells give greater contributions to the $\mathrm{AB}+\mathrm{RI}$ process below and up to $T_{\max }$, as it can be observed in Figures 5.2 and 5.3; above that value, the DDCSs of the outer shells decrease abruptly, while the inner shells present a much smoother transition and tend to prevail at higher photon energies.

Moreover, in the region above $T_{\max }$, the results obtained with DHFS VDs are clearly larger than those calculated from the hydrogenic ones, again on account of the stronger high momentum tails present in the former (see Section 3.1.2). For photon energies lower than $T_{\max }$, the calculations with the hydrogenic VDs overshoot the ones ensuing from the DHFS VDs, contrary to the case of the SEB DDCSs, where the two converge to quite similar values (compare Figures 5.2, 5.3, and 5.4 with Figures 5.5, 5.6, and 5.7). The energy $T_{\max }$ thus marks the region where this inversion occurs, and the effect is more evident in the outer shells.

When the contributions of all shells are summed, as shown in Figure 5.7, the mentioned features and in particular the inversion of the larger DDCSs obtained with both VDs models are even more noticeable, especially in the case of $4 \mathrm{MeV}$ proton energy. 

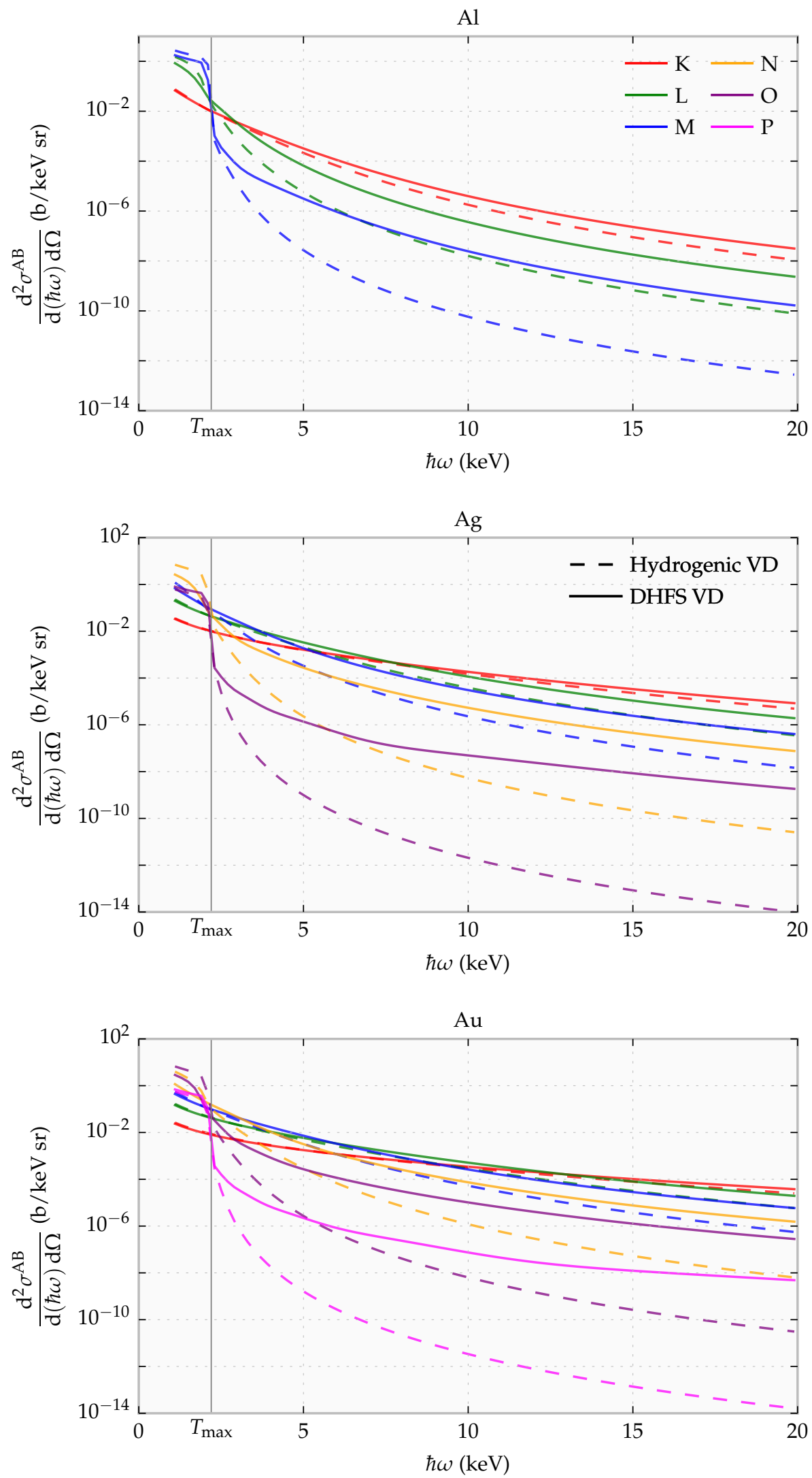

Figura 5.5: $\mathrm{AB}+\mathrm{RI} \mathrm{DDCS}$ for protons impinging on $\mathrm{Al}, \mathrm{Ag}$, and $\mathrm{Au}$ with a kinetic energy of $1 \mathrm{MeV}$ radiating photons with an emission angle of $90^{\circ}$. Each line is the sum over the corresponding (sub)shells. Calculations employing hydrogenic VDs (dashed lines) and DHFS VDs (solid lines) are shown. 

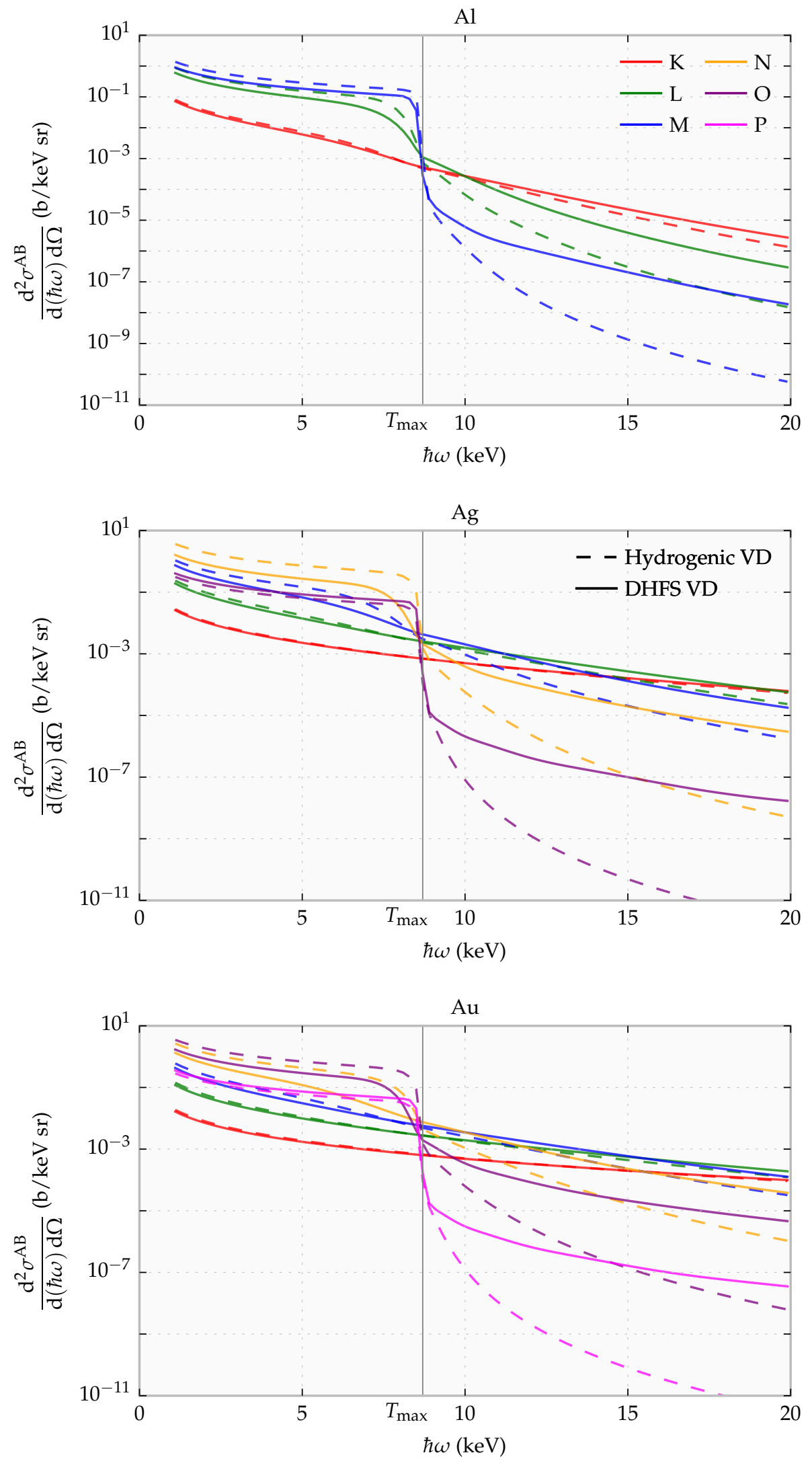

Figura 5.6: $\mathrm{AB}+\mathrm{RI} \mathrm{DDCSs}$ for protons impinging on $\mathrm{Al}, \mathrm{Ag}$, and $\mathrm{Au}$ with a kinetic energy of $4 \mathrm{MeV}$ radiating photons with an emission angle of $90^{\circ}$. Each line is the sum over the corresponding (sub)shells. Calculations employing hydrogenic VDs (dashed lines) and DHFS VDs (solid lines) are shown. 

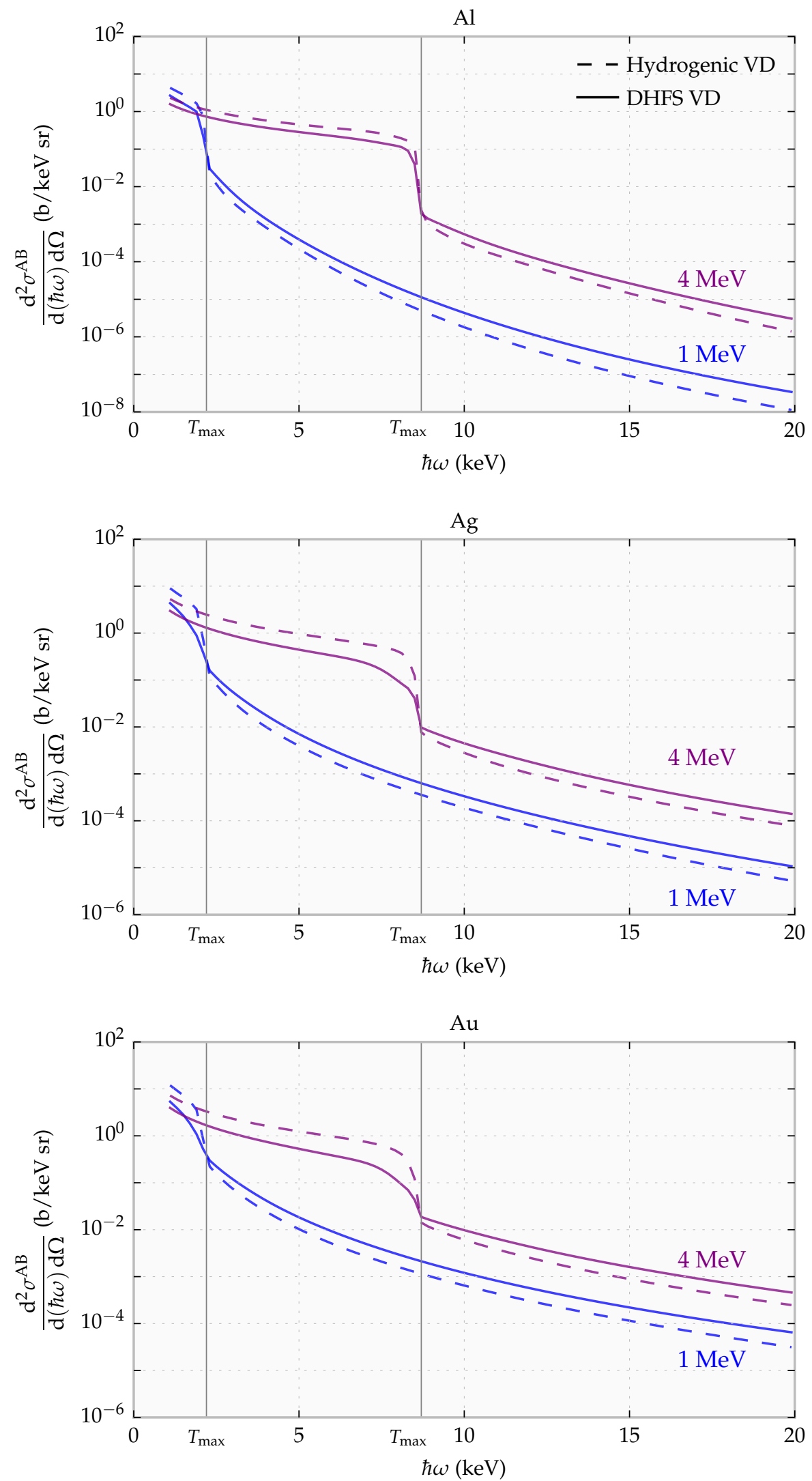

Figura 5.7: $\mathrm{AB}+\mathrm{RI} \mathrm{DDCS}$ for protons impinging on $\mathrm{Al}, \mathrm{Ag}$, and $\mathrm{Au}$ with kinetic energies of $1 \mathrm{MeV}$ and $4 \mathrm{MeV}$ radiating photons with an emission angle of $90^{\circ}$. Calculations employing hydrogenic VDs (dashed lines) and DHFS VDs (solid lines) are shown. 


\subsection{Comparison with experimental data}

To complement the investigation of the effects of the DHFS VDs on the SEB and $\mathrm{AB}+\mathrm{RI} \mathrm{DDCSs}$, the theoretical results are compared to the experimental spectra discussed in the previous Chapter 4. The proton bremsstrahlung DDCS is composed of the sum of the five processes reviewed in Chapter 2. Therefore, additionally to the SEB and the AB+RI DDCSs, those for NB and QFEB have been calculated for the cases presented in Table 4.1.

The proton bremsstrahlung DDCSs are shown for the case of the Au target bombarded with $3 \mathrm{MeV}$ protons in Figure 5.8 individually for each process in panel (a) (using different colors) and summed together in panel (b). As addressed in the previous section, the SEB and the $\mathrm{AB}+\mathrm{RI}$ DDCSs are calculated with the hydrogenic and DHFS VDs (plotted with dashed and continuous lines, respectively). The QFEB DDCSs, on the other hand, is calculated using Equation (2.3.48), which is the velocity component in the $z$ direction for an hydrogenic $1 \mathrm{~s}$ orbital, as discussed in Subsection 2.3.3. Such an approximation is not important since the dominant contributions come from the $\mathrm{AB}+\mathrm{RI}$ and $\mathrm{SEB}$ processes (see gain Figure 5.8a), while the QFEB mechanism only affects the total DDCS at very small energies of the photon. The NB DDCS is smaller than those from all the other processes: although it surpasses the SEB DDCS with hydrogenic VDs above photon energies of around $22 \mathrm{keV}$, it still remains below those with DHFS VDs up to roughly $34 \mathrm{keV}$. Note that at both 22 and $34 \mathrm{keV}$ the cross sections are too small to have any practical relevance.

In the case of 3 and $2 \mathrm{MeV}$ protons, $T_{\max }=6.5$ and $4.4 \mathrm{keV}$, respectively. When the DDCSs from all bremsstrahlung processes are summed, as shown in Figure 5.8b, significant contributions from the $\mathrm{AB}+\mathrm{RI}$ and SEB DDCSs below $T_{\max }$ form a strong shoulder in the curve that rapidly decreases towards higher photon energies. When hydrogenic VDs are employed, a sharp transition appears in the shoulder, which is smoothed out by DHFS VDs. As a matter of fact, the two curves cross each other close to $T_{\max }$, and the total bremsstrahlung DDCS calculated with the DHFS VDs remains substantially higher above $T_{\max }$.

In order to compare the results shown in Figure 5.8 with the PIXE spectrum measured at LAMFI (see Table 4.1), the bremsstrahlung DDCS must be corrected for the attenuation of the photons in the target material, the detector efficiency, and then convoluted with the detector response function, as shown in Equation (4.3.1).

As discussed in Section 4.1, because of the angle between the direction of incidence of the proton beam and the position of the detector relative to the target, the attenuation of the photons produced in the target can have significant impact on the measurements. The attenuation calculated as in Figure 4.5 is applied to the bremsstrahlung DDCS from Figure 5.8b. When considering this effect, the DDCS is reduced as depicted in Figure 5.9, where the red and 
(a)



(b)

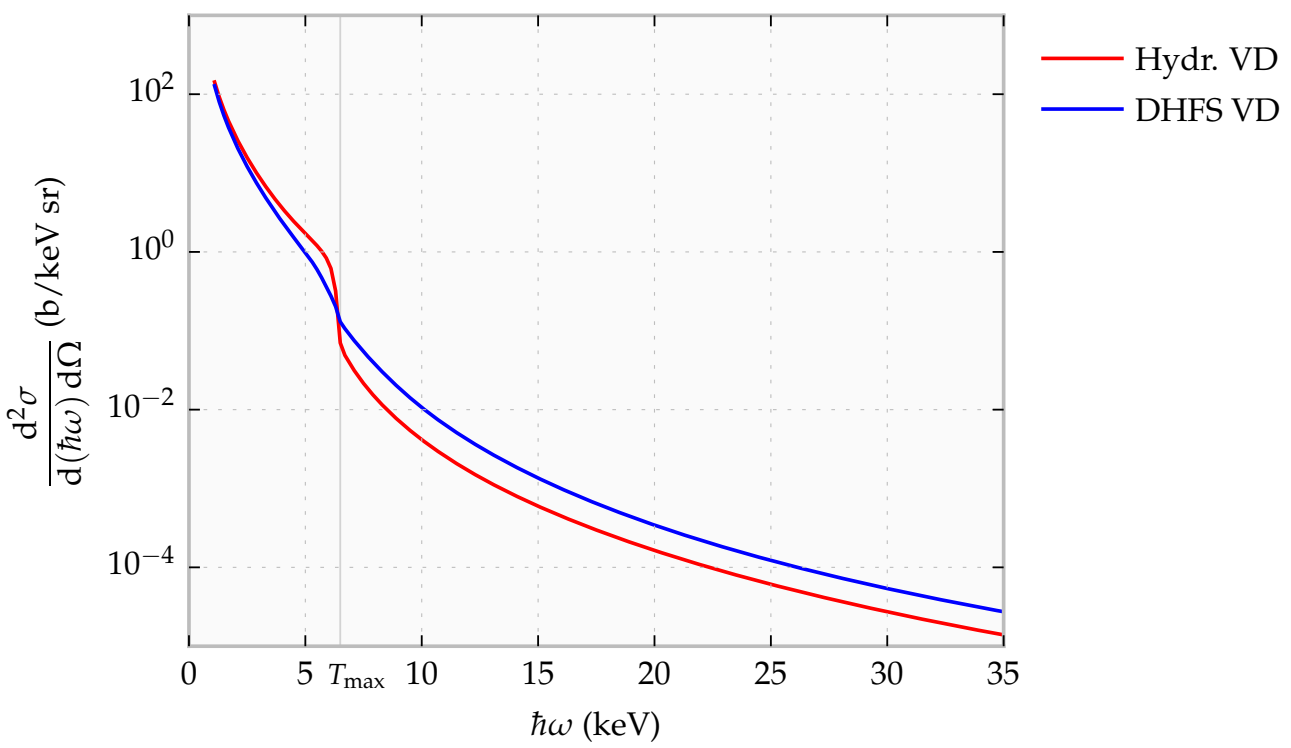

Figura 5.8: Contributions of each process to the DDCS (a) and total DDCS (b) for proton bremsstrahlung. The case considered is that of protons impinging on an Au target with a kinetic energy of $3 \mathrm{MeV}$ radiating photons with an angle of $90^{\circ}$. The hydrogenic (dashed lines) and DHFS (solid lines) VDs are also compared.

green curves show the primary and attenuated DDCSs, respectively. Only the case of hydrogenic VDs up to $9 \mathrm{keV}$, where the attenuation is stronger, is shown for a better visualization. The same calculation is applied to the spectrum with the DHFS VDs for further processing.

Afterwards, the detector efficiency and the convolution with its response function can 


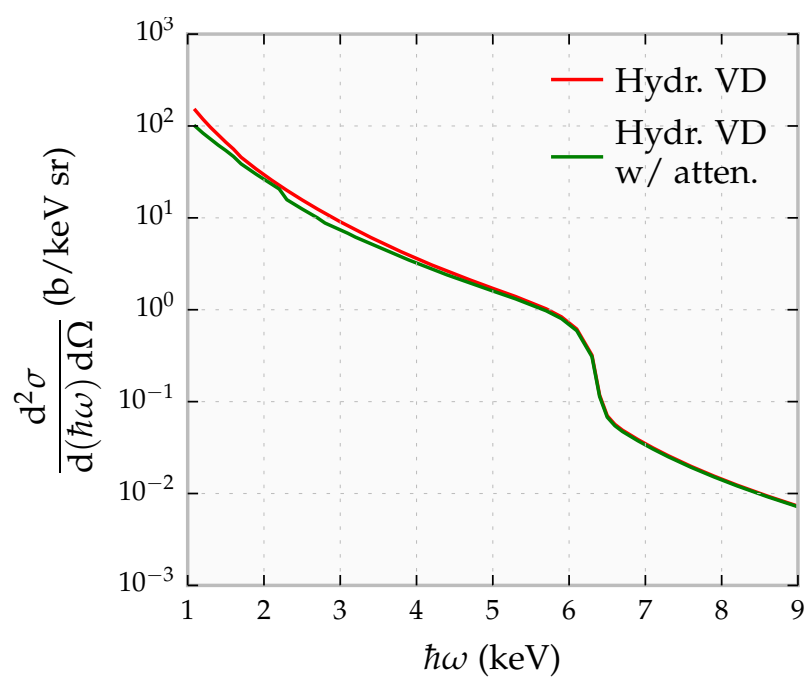

Figura 5.9: Attenuation of the calculated total DDCS for proton bremsstrahlung of Figure 5.8 by photon absorption inside the target. The red and green lines represent the primary and attenuated DDCS, respectively.

be applied, as described in the Subsection 4.3, so the DDCSs can be converted to a counting rate as a function of the energy of the photon. The parameters required for such a procedure are described in the previous Chapter 4: they have been estimated by fitting the most intense and most promptly recognizable characteristic lines, as discussed in Subsection 4.3. The resulting bremsstrahlung spectrum is shown in Figure 5.10, in comparison to the PIXE spectrum R040094X.

Figure 5.10 depicts the results for both calculations using the hydrogenic (red curve) and DHFS (blue line) VDs. As expected, the spectrum is strongly attenuated both at low and high energies of the photon, mainly below $2 \mathrm{keV}$ and above $8 \mathrm{keV}$. This effect is due to the detector efficiency, which includes the attenuation from the beryllium window, $f_{\mathrm{Be}}$, at lower photon energies, and the intrinsic efficiency, $\varepsilon_{\mathrm{i}}$, at higher photon energies, see Section 4.2.2. The bremsstrahlung yield calculated with the hydrogenic VD is generally greater than that obtained from the DHFS one over the photon energy interval where the attenuation from the detector efficiency is smaller. Above $T_{\max }$, most of the background counts registered in the spectrum are due to the pile-up effect, as will be shown below. Furthermore, the characteristic x-ray yield must be added in order to reproduce the spectrum above $T_{\max }$.

As a next step, the characteristic peaks shown in Figure 4.10 are added to the calculated spectrum of Figure 5.10, so that the main aspects of the PIXE spectrum, i.e. the emission lines plus the bremsstrahlung contributions, are taken into account. The characteristic emission lines have been corrected for the self-attenuation in the target and the detector efficiency and then convoluted with the response function of the detector in the same way as the calculated 


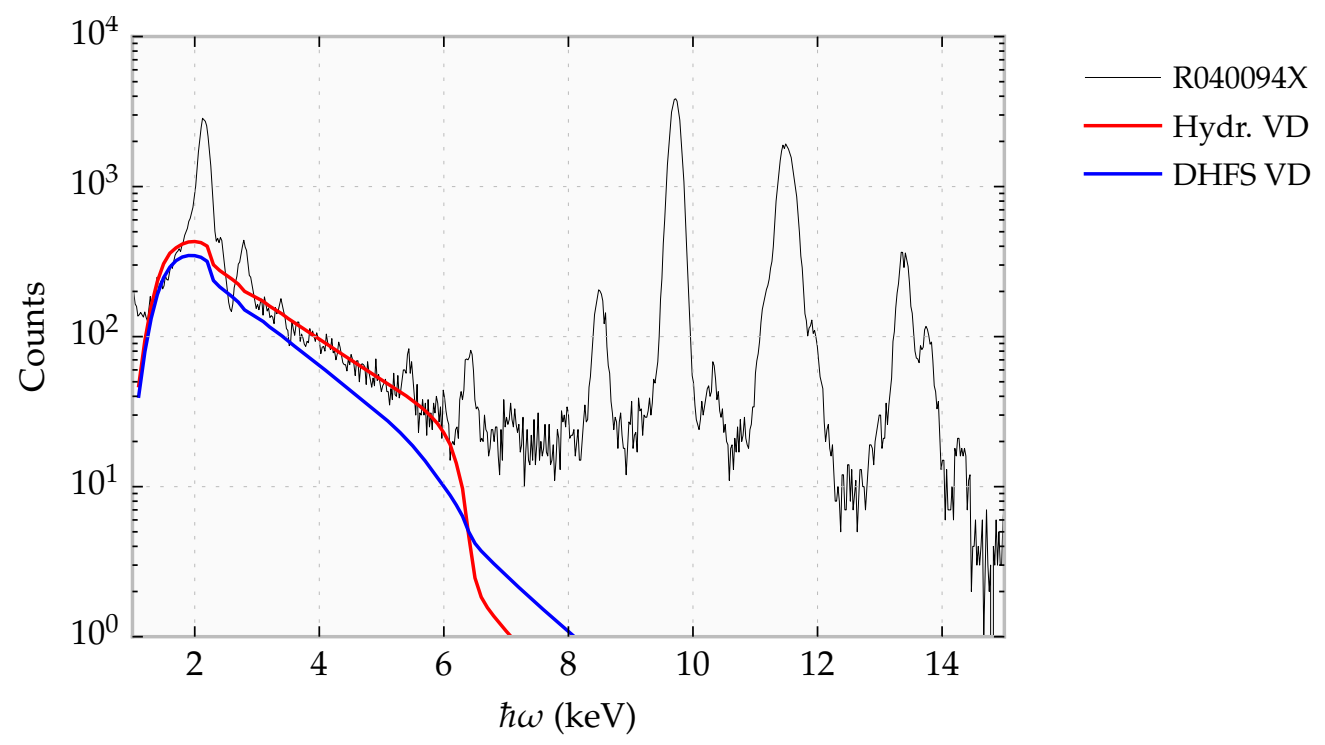

Figura 5.10: Measured PIXE spectrum R040094 (black line) and calculations with the hydrogenic (red line) and DHFS (blue line) VDs. The effect of self-attenuation in the target, the detector efficiency, and the convolution with the detector response function have been included in the theoretical results.

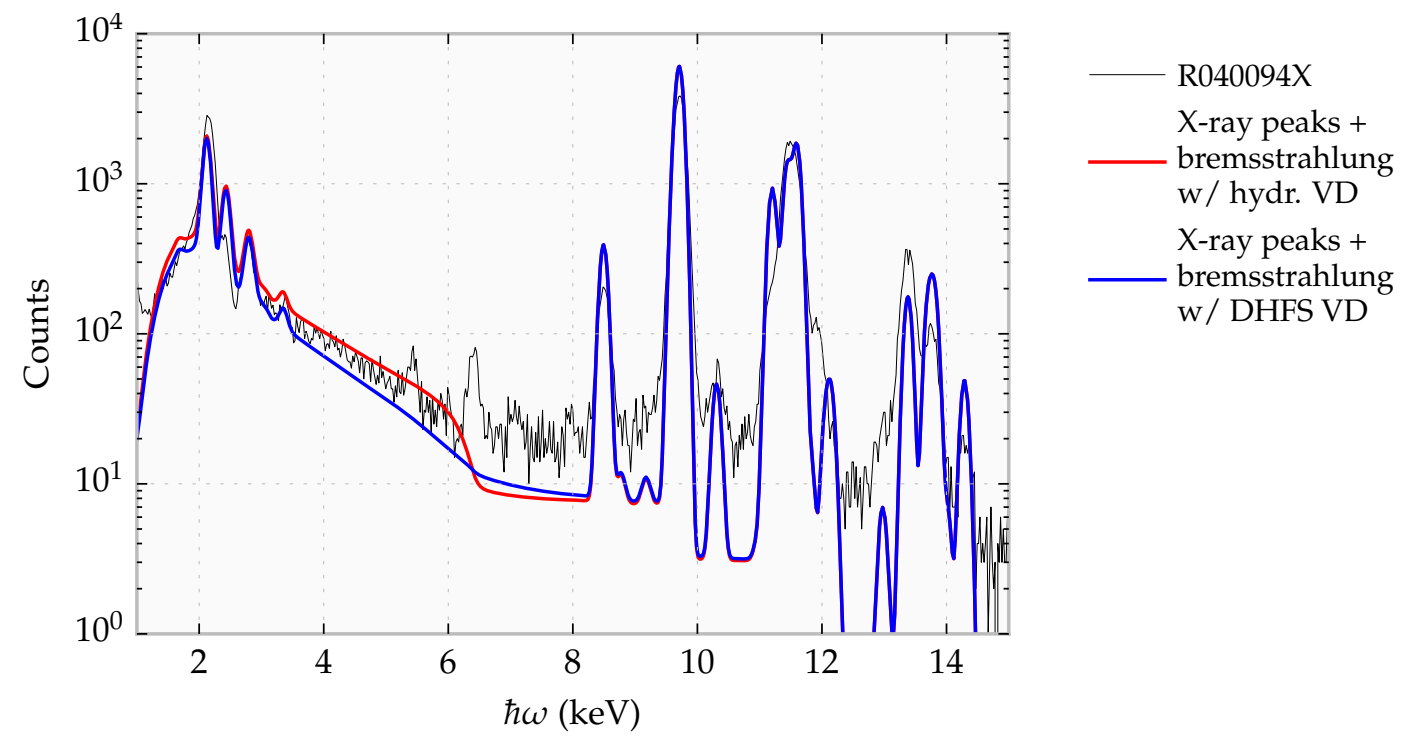

Figura 5.11: Measured PIXE spectrum R040094X (black line) and calculated characteristic peaks (see Figure 4.10) plus bremsstrahlung. The hydrogenic (red line) and DHFS (blue line) VDs are compared. The effect of self-attenuation in the target, the detector efficiency, and the convolution with the detector response function have been included in the theoretical results.

proton bremsstrahlung.

At this stage, the effect of pile-up can be calculated from the resulting theoretical spectrum shown in Figure 5.9, as described in Section 4.2.3. In such a case, the terms $P_{i}$ and $P_{j}$ in Equation (4.2.13) correspond to the yield in channels $i$ and $j$ divided by the yield 


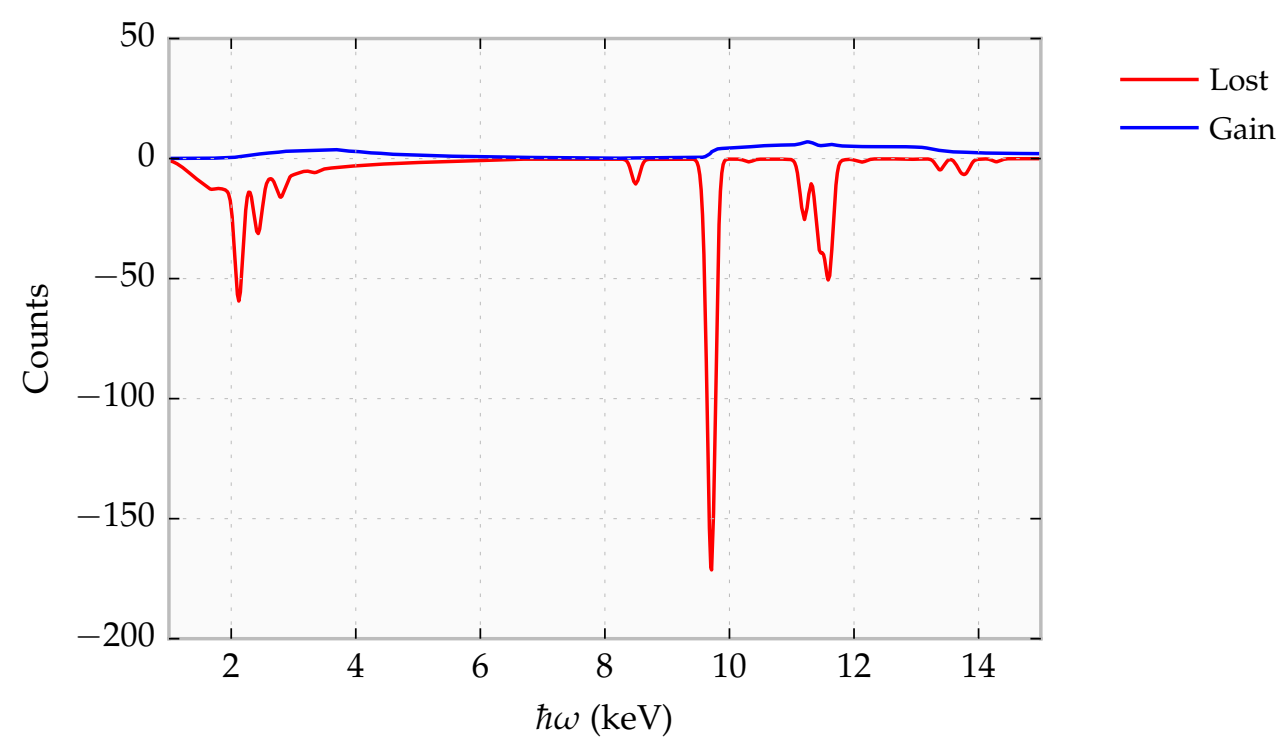

Figura 5.12: Pile-up effect calculated for the theoretical spectrum of Figure 5.11 (only the hydrogenic VDs are considered for sake of illustration). The red line represents the lost counts in the corresponding channel, while the blue line shows the gained ones.

integral, and $N_{t}$ is the yield integral divided by the measurement time of the experiment. The parameter $T_{\text {shape }}$ is a characteristic of the amplifier and here the value of $4 \mu \mathrm{sec}$ has been adopted according to the specifications given by the manufacturer of the equipment in use at the LAMFI laboratory. Additionally, the parameter $T_{\text {rise }}$ was adjusted to $300 \mu \mathrm{sec}$ in order to obtain reasonable results for the characteristic x-rays. With these choices, it is possible to calculate the values of $L_{i j k, i}, L_{i j k, j}$ and $G_{i j k, k}$, see Equation (4.2.13), as shown in Figure 5.12. In this plot, the red line corresponds to the lost counts per channel, while the blue line shows the gained pulses.

Finally, the lost and gained counts, illustrated in Figure 5.12, can be added to the total spectrum, so that the final spectrum takes into account the pile-up effect. The result is displayed in Figure 5.13 for the hydrogenic VDs. It can be seen that some counts move from the area underneath the peaks to the region between them and beyond the energy range of the bremsstrahlung spectrum. For energies above $10 \mathrm{keV}$, the pile-up effect gives a more important contribution than the bremsstrahlung itself.

Figures 5.14 and 5.15 show the final results for the PIXE spectra R040094X and R040097X, corresponding to impinging protons with energies of 3 and $2 \mathrm{MeV}$, respectively. In both cases, it can be noted that most of the aspects of the spectra are well reproduced by the theory. In principle, better agreement with the experimental spectra is expected with an improved calibration of the detector. However, these results already suggest that the bremsstrahlung calculations behave as expected, particularly for the region between $4-8 \mathrm{keV}$, where 


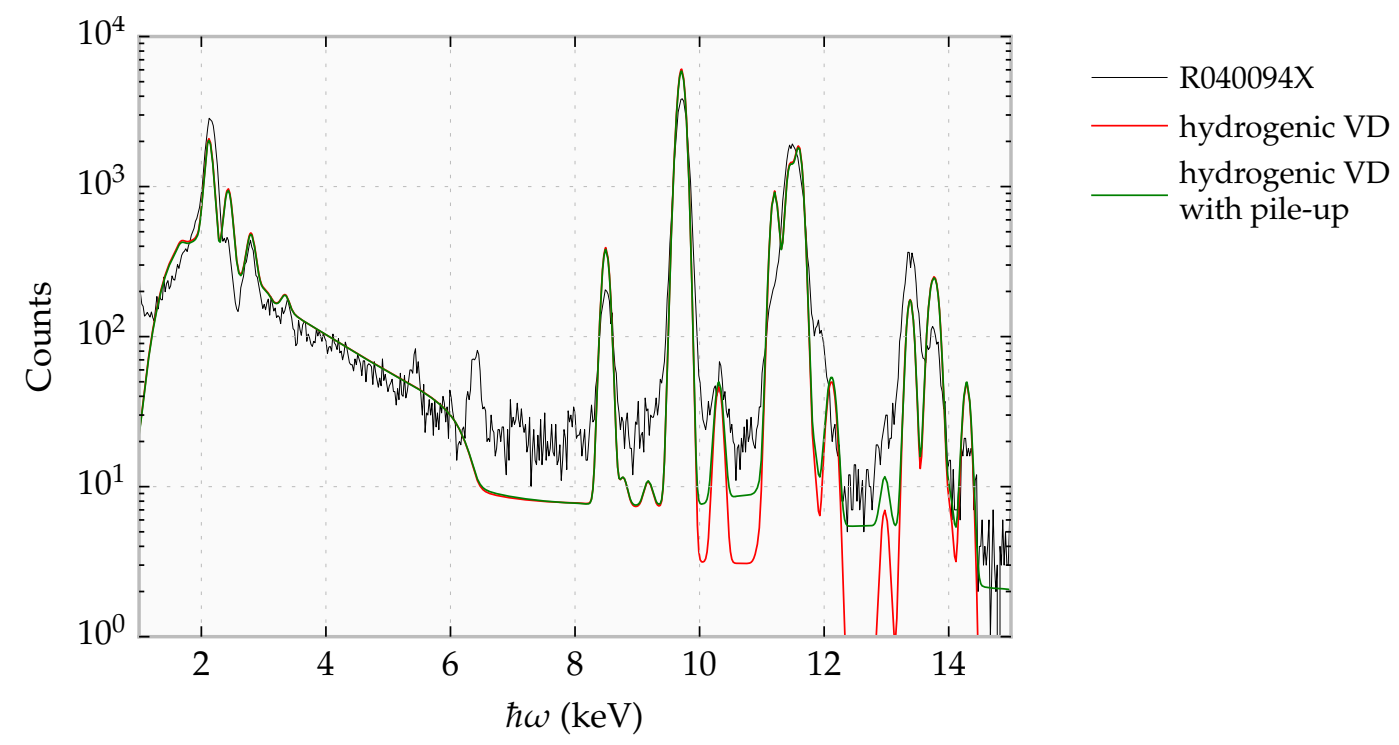

Figura 5.13: Comparison between the calculated spectrum without the pile-up effect (red line, same of Figure 5.11), the calculated spectrum taking into account that effect (green line), and the measured PIXE spectrum R040094X (only the hydrogenic VDs are considered for sake of illustration).

the bremsstrahlung contributions mostly explain the continuous background.

The results obtained with hydrogenic and DHFS VDs are represented with red and blue lines, respectively, in Figures 5.14 and 5.15. By comparing the two curves, in both figures, particularly in the energy region where the bremsstrahlung contribution is more prominent, it is possible to see that the spectrum calculated from the DHFS VDs is closer to the experimental data than that obtained using the hydrogenic VDs. Actually, the spectrum obtained from the hydrogenic VDs surpasses the data in the energy region below $6.5 \mathrm{keV}$, which corresponds to $T_{\max }$ for $E_{\mathrm{p}}=3 \mathrm{MeV}$ (see the red curve in Figure 5.14). Beyond that energy, the spectrum rapidly decreases due the sharp transition in the bremsstrahlung DDCS at $T_{\max }$, and it becomes smaller than the experimental spectrum. On the other hand, the blue curve, denoting to the calculations from the DHFS VDs, is closer to the experimental data in the same energy range. Because the transition at $T_{\max }$ in the corresponding bremsstrahlung DDCS is less prominent, the spectrum is smoother in that region, resulting in the mentioned better agreement with the experiment.

The same comparison between the two VD models can be carried out for $E_{\mathrm{p}}=2 \mathrm{MeV}$, as shown in Figure 5.15. As a matter of fact, this case is even clearer, since the spectrum calculated with the DHFS VDs presents a smoother transition around the energy $T_{\max }$, located in a more convenient region of the spectrum (less distorted by the response function of the detector), which is in better agreement with the experimental data.

In conclusion, with the procedures described above, quantitative comparisons between 
the theoretical calculations of the bremsstrahlung DDCSs and the experimental data could be performed in order to understand the impact of the VDs in the continuous part of the PIXE spectrum.

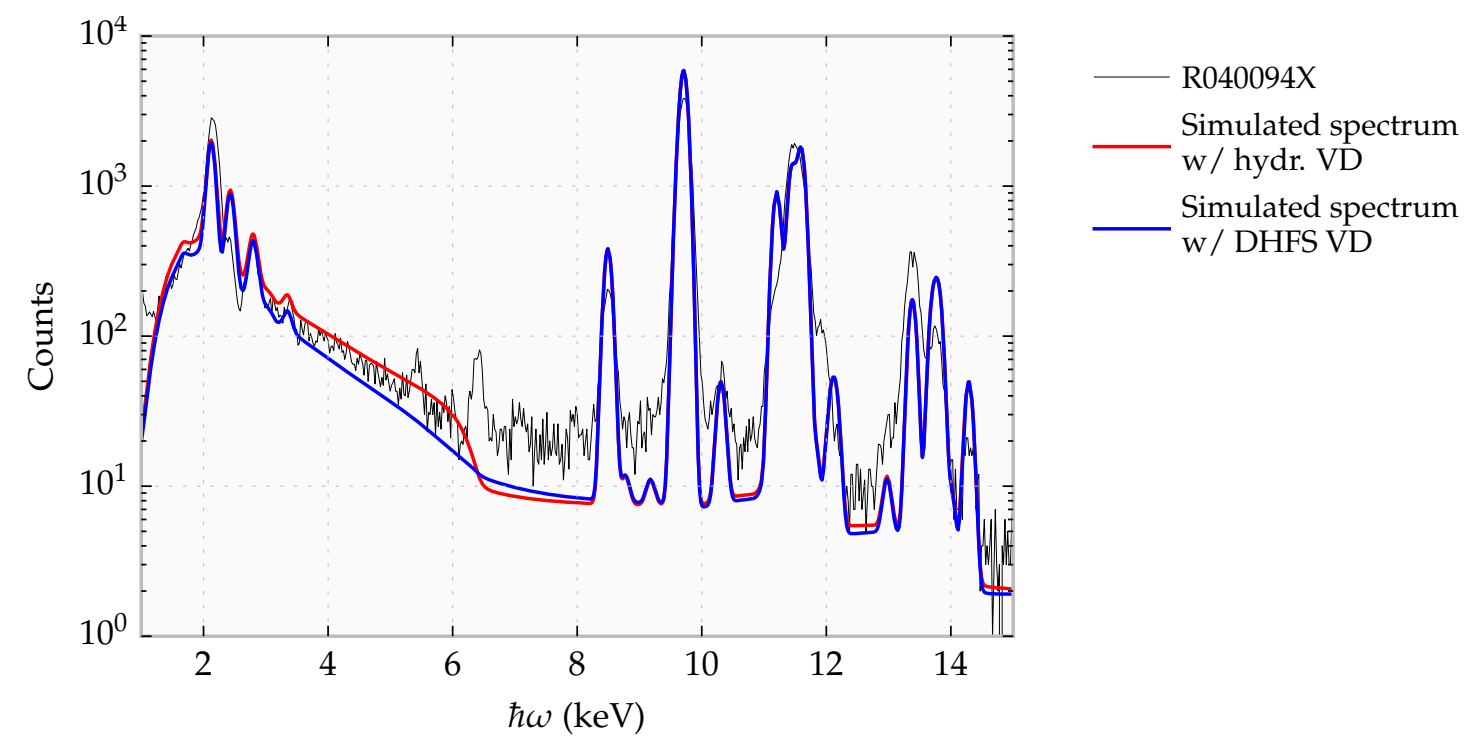

Figura 5.14: Comparison between the calculated and measured PIXE spectrum R040094X, for an $\mathrm{Au}$ target and a $3 \mathrm{MeV}$ proton beam. All experimental effects mentioned in the text (namely, self-attenuation in the target, detector efficiency and its response function, and pile-up in the electronic chain) have been included in the theory. The hydrogenic (red line) and DHFS (blue line) VDs are also compared.

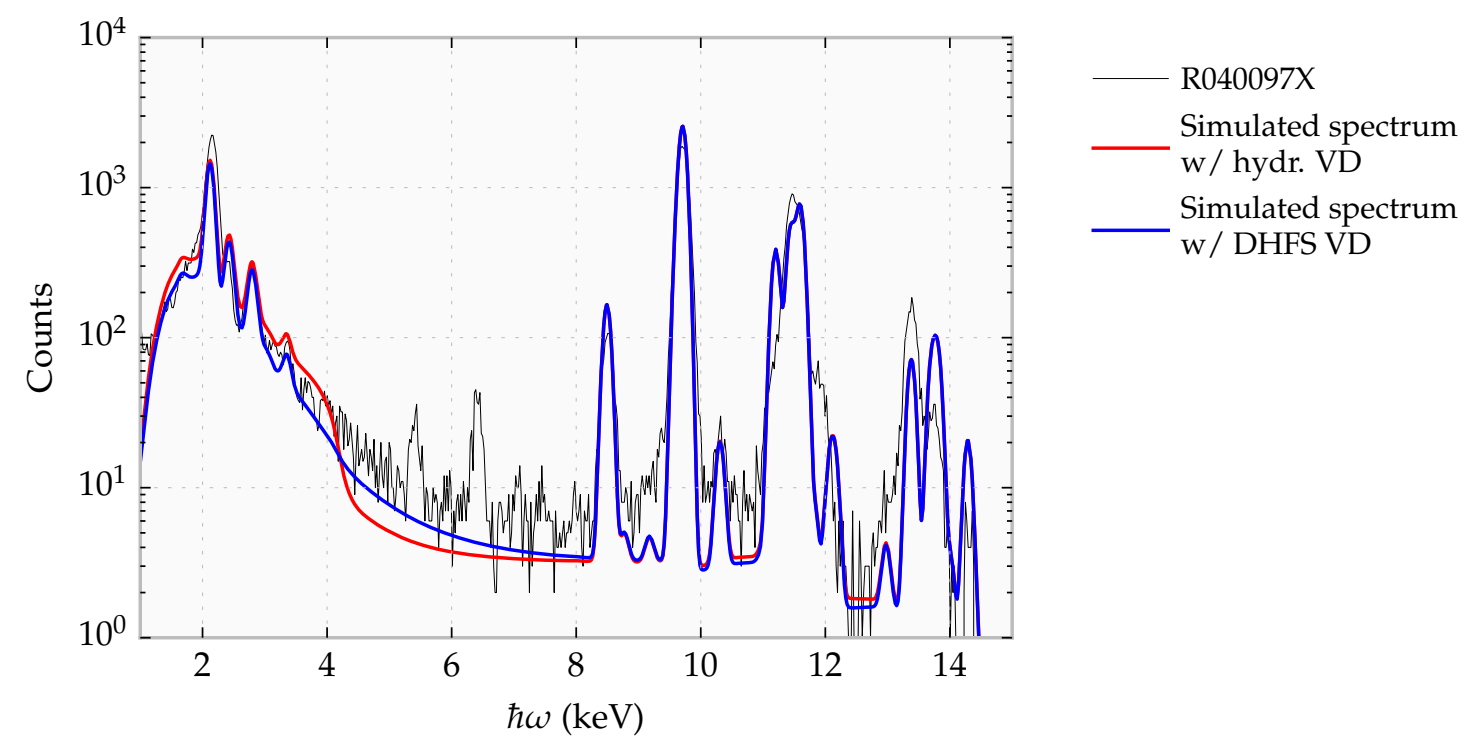

Figura 5.15: Same as Figure 5.14, but for the comparison with measured PIXE spectrum R040097X, for an $\mathrm{Au}$ target and a $2 \mathrm{MeV}$ proton beam. 


\section{Summary}

The ionization, SEB, and AB+RI DDCSs, obtained using the analytical hydrogenic and numerical DHFS VDs, have been compared to investigate the importance of this aspect of the calculations. The latter approach makes it possible to consider the relativistic effects for the inner shells and the screening of the Coulomb field for the outer ones. Additionally, the corresponding analytical expressions for the hydrogenic VDs of the K, L, and M (sub)shells have been implemented in this work.

The cross sections have been calculated for aluminum, silver, and gold targets, with proton energies of 1 and $4 \mathrm{MeV}$. The results for the ionization cross sections are shown to agree with previous similar studies, where the curves from both VDs reveal a crossing point, whose location in proton energy depends on the element and shell under consideration. The ionization cross sections have been compared with tabulations prepared with the PWBA and ECPSSR theories, to show the discrepancies associated with different approaches.

The SEB and AB+RI DDCSs exhibit relevant differences depending on the VDs used in the calculations, especially for the outer shells. When summing the results from all shells, the shoulder in the curve expected near the maximum energy, $T_{\max }$, that can be transferred by the proton to an electron at rest, is smoother in the calculations using the DHFS VDs. This remains visible in the total bremsstrahlung DDCSs, with the contributions from all processes added together.

The bremsstrahlung calculations have been compared to experimental PIXE spectra obtained at LAMFI, USP. The target consisted of a self-supporting gold film, with a thickness of $26.34 \mu \mathrm{g} / \mathrm{cm}^{2}$. The energies of the incident proton beam have been set to 2 and $3 \mathrm{MeV}$ and the angle of incidence to $7^{\circ}$. The angle of detection of the x-rays has been fixed at $90^{\circ}$.

In order to perform the comparison between the theoretical DDCSs and the experimental spectra, the characteristic x-ray lines, the self-attenuation in the target, the detector effects, and some aspects of the electronics of the detection system used at LAMFI have been considered. The calibration of the detector has been performed by adjusting the parameters of the response function convoluted with the emission lines of $\mathrm{Au}$, so that the measured characteristic peaks could be visually well reproduced. Then, the experimental effects have been applied to the calculated bremsstrahlung DDCSs and characteristic x-rays.

The calculations are in better agreement with the data when the DHFS VDs are employed. The spectra calculated using the hydrogenic VDs overestimate and underestimate the bremsstrahlung production below and above $T_{\max }$, respectively, because of the presence of a sharp transition. Near $T_{\max }$, the spectra calculated with the DHFS VDs present a less pronounced drop, as compared to the case of the hydrogenic VDs, and are closer to the behavior of the experimental spectra in that energy region. 


\section{Capítulo 6}

\section{Conclusions}

The five processes of proton-induced bremsstrahlung, namely the secondary electron bremsstrahlung (SEB), the atomic bremsstrahlung (AB), the radiative ionization (RI), the quasi-free electron bremsstrahlung (QFEB), and the nuclear bremsstrahlung (NB), have been reviewed. An alternative formulation for the SEB DDCS has been proposed, which expresses the integration over the energy loss of the secondary electron in terms of an exponential integral. This approach reduces the complexity for numerical calculations of the SEB DDCS and has been shown to be consistent with the equations derived by Ishii et al. (1976).

Moreover, the present work proposes to adopt numerical calculations with the DHFS method as an alternative to the analytical hydrogenic expressions, usually employed in current publications. The VD from the DHFS wave functions used for that purpose have been calculated by J. M. Fernández-Varea.

The ionization, SEB, and AB+RI DDCSs have been implemented using both the numerical DHFS and the analytical hydrogenic VDs, allowing for a comparison between the results. Moreover, the correct hydrogenic VDs in the case of the K, L, and M (sub)shells have been employed, as opposed to the frequently adopted approximation of using the 1s hydrogenic VD for all shells. For the outer shells, however, the DDCSs have been calculated using the 1s hydrogenic VD, due to the increasing complexity of the equations.

The results for the total ionization cross sections present a crossing point between the curves calculated with the DHFS VDs and the hydrogenic VDs. The location of that point in the energy range of the proton depends on the element and shell. Above the crossing point, the cross sections from the DHFS VDs are higher than the ones from the hydrogenic VDs. This behavior is in agreement with previous publications which covered the outer shells in different elements (Mukoyama, 2015, 2017, 2018).

In the SEB DDCSs, it has been noted that the results from the DHFS VDs are greater than those from the hydrogenic VDs above the maximum energy $T_{\max }$, that the proton can 
transfer to an electron of the target atom initially ta rest, mainly in the outer shells, which is consistent with the results observed for the ionization cross sections. Below that energy, however, the DDCSs from both models tend to be closer. Nonetheless, the sharp drop present in the SEB DDCSs near $T_{\max }$ is smoother in the calculations with the DHFS VDs. This behavior remains clearly visible even when the contributions from all the occupied shells are summed. The above results have been published by Ferro et al. (2020) and presented at the IBA conference (2019) as a poster.

For the AB+RI DDCSs, similar results have been found, where the DDCSs calculated using the DHFS VDs are higher than the hydrogenic ones above $T_{\max }$, again especially in the outer shells. In this case, however, the two curves cross each other near the energy $T_{\max }$, and the DDCSs from the hydrogenic VDs become larger than those from the DHFS calculations. This inversion can be clearly observed in the results summed over all the occupied shells.

Additionally, two PIXE spectra have been obtained, so that the bremsstrahlung calculations can be confronted with experiment. The measurements have been carried out by T. F. da Silva, at LAMFI, USP, for a gold target and energies of the proton beam of 2 and $3 \mathrm{MeV}$. In order to compare the theoretical bremsstrahlung with the PIXE data, several detector effects have been reviewed. The response function of the device used at LAMFI has been modeled and calibrated with the help of T. F. da Silva and it has been applied to the calculated spectra, containing the five proton-induced bremsstrahlung cross sections and the characteristic x-rays cross sections.

The results showing the sum of all the bremsstrahlung processes maintain the characteristic of a smoother transition around $T_{\max }$ in the DDCSs with the DHFS VDs, as observed separately for the SEB and the AB+RI DDCSs. After the convolution with the response function of the detector, it has been noted that the calculations using the DHFS VDs are closer to the data than the ones obtained with the hydrogenic VDs. Mainly in the region near $T_{\max }$, where the spectra sharply decrease, the less abrupt drop shown in the results with the DHFS VDs is in better agreement with the experiment. This conclusion is even more valid in the case of $2 \mathrm{MeV}$ protons.

Finally, despite the good results observed with the implementation of the DHFS VDs and an alternative expression for the SEB DDCS, more experimental measurements, for other target elements and proton energies, are desirable for establishing the accuracy of the theoretical calculations. As a matter of fact, the electron bremsstrahlung DDCSs employed in the SEB calculations is formulated in the non-relativistic dipole approximation without screening. This aspect can be improved by employing the numerical results obtained by Kissel et al. (1983) and by Tseng and Pratt (1971) on the basis of partial-wave expansions. Furthermore, the choice of the thickness of the targets must consider the range of the secondary electrons, as has been discussed in the present work. 




\section{Referências Bibliográficas}

Abramowitz, M. and Stegun, I. A. (1970). Handbook of mathematical functions : with formulas, graphs, and mathematical tables. Dover Publications.

Alder, K., Bohr, A., Huus, T., Mottelson, B., and Winther, A. (1956). Study of Nuclear Structure by Electromagnetic Excitation with Accelerated Ions. Reviews of Modern Physics, 28(4):432-542.

Anholt, R. (1985). X rays from quasimolecules. Reviews of Modern Physics, 57(4):995-1053.

Application Software Group (1996). CERNLIB - CERN Program Library Short Writeup, page 414. CERN, Geneva.

Barradas, N. P. and Reis, M. A. (2006). Accurate calculation of pileup effects in PIXE spectra from first principles. X-Ray Spectrometry, 35(4):232-237.

Berger, M. J., Coursey, J. S., Zucker, M. A., and Chang, J. (2017). Stopping-Power and Range Tables for Electrons, Protons, and Helium Ions.

Bethe, H. (1930). Zur Theorie des Durchgangs schneller Korpuskularstrahlen durch Materie. Annalen der Physik, 397(3):325-400.

Bethe, H. (1932). Bremsformel für Elektronen relativistischer Geschwindigkeit. Zeitschrift für Physik, 76:293.

Bethe, H. and Heitler, W. (1934). On the Stopping of Fast Particles and on the Creation of Positive Electrons. Proceedings of the Royal Society A: Mathematical, Physical and Engineering Sciences, 146(856):83-112.

Bethe, H. and Salpeter, E. E. (1957). Quantum Mechanics Of One-And Two-Electron Atoms. Springer-Verlag, New York.

Bonsen, T. and Vriens, L. (1970). Angular distribution of electrons ejected by charged particles. I. Ionization of He and H2 by protons. Physica, 47:307-319. 
Brandt, W. and Lapicki, G. (1979). L -shell Coulomb ionization by heavy charged particles. Physical Review A, 20(2):465-480.

Bransden, B. H. and Joachain, C. J. (2003). Physics of Atoms and Molecules. Pearson.

Bransden, B. H., Joachain, C. J. C. J., and Bransden, B. H. (2000). Quantum mechanics. Prentice Hall.

Brent, R. P. (2010). MP users guide.

Campbell, J. L., Hopman, T. L., Maxwell, J. A., and Nejedly, Z. (2000). The Guelph PIXE software package III: Alternative proton database. Nuclear Instruments and Methods in Physics Research Section B: Beam Interactions with Materials and Atoms, 170(1-2):193-204.

Catlow, G. W. and McDowell, M. R. C. (1967). A classical model for electron and proton impact ionization. Proceedings of the Physical Society, 92(4):875-879.

Chu, T., Ishii, K., Yamadera, A., Sebata, M., and Morita, S. (1981). Quasifree electron bremsstrahlung induced by 20-MeV-proton impact. Physical Review A, 24(4):1720-1725.

Cohen, D. D., Crawford, J., and Siegele, R. (2015). K, L, and M shell datasets for PIXE spectrum fitting and analysis. Nuclear Instruments and Methods in Physics Research Section B: Beam Interactions with Materials and Atoms, 363:7-18.

Cohen, D. D. and Harrigan, M. (1985). K- and L-shell ionization cross sections for protons and helium ions calculated in the ecpssr theory. Atomic Data and Nuclear Data Tables, 33(2):255-343.

Fermi, E. (1940). The Ionization Loss of Energy in Gases and in Condensed Materials. Physical Review, 57(6):485-493.

Ferro, R. M., Mangiarotti, A., and Fernández-Varea, J. M. (2020). Calculation of secondary electron bremsstrahlung in the binary encounter approximation using Dirac-Hartree-Fock-Slater velocity distributions. Nuclear Instruments and Methods in Physics Research Section B: Beam Interactions with Materials and Atoms, 478:70-79.

Folkmann, F., Gaarde, C., Huus, T., and Kemp, K. (1974). Proton induced X-ray emission as a tool for trace element analysis. Nuclear Instruments and Methods, 116(3):487-499.

Folkmann, F., Groeneveld, K. O., Mann, R., Nolte, G., Schumann, S., and Spohr, R. (1975). Continuous electron energy spectra ejected from solid carbon targets bombarded with light and heavy ions. Zeitschrift für Physik, 275(3):229-233. 
Gardner, R. P. and Wielopolski, L. (1977). A generalized method for correcting pulse-height spectra for the peak pile-up effect due to double sum pulses: Part I. Predicting spectral distortion for arbitrary pulse shapes. Nuclear Instruments and Methods, 140(2):289-296.

Hansen, J. S., Mcgeorge, J. C., Nix, D., Schmidt-Ott, W. D., Unus, I., and Fink, R. W. (1973). Accurate efficiency calibration and properties of semiconductor detectors for low-energy photons. Nuclear Instruments and Methods, 106:365-379.

Haug, E. and Nakel, W. (2004). The elementary process of Bremsstrahlung. World Scientific.

Heitler, W. (1954). The quantum theory of radiation. Clarendon Press, 3 edition.

Hubbell, J. (1969). Photon cross sections, attenuation coefficients and energy absorption coefficients. National Bureau of Standards Report NSRDS-NBS29, Washington DC.

ICRU (1984). Stopping Powers for Electrons and Positrons (Report 37). Technical report, ICRU Bethesda, MD.

Ishii, K. (2006). Continuous X-rays produced in light-ion-atom collisions. Radiation Physics and Chemistry, 75(10):1135-1163.

Ishii, K., Matsuyama, S., Yamazaki, H., Kikuchi, Y., Momose, G., and Satoh, T. (2008). Atomic bremsstrahlung of $\mathrm{Al}, \mathrm{Ag}$ and $\mathrm{Au}$ targets bombarded with $1.5 \mathrm{MeV}$ protons. $X-R a y$ Spectrometry, 37(2):121-124.

Ishii, K. and Morita, S. (1984). Continuum x rays produced by light-ion-atom collisions. Physical Review A, 30(5):2278-2286.

Ishii, K. and Morita, S. (1990). Continuous Backgrounds in PIXE. International fournal of PIXE, 01(01):1-29.

Ishii, K., Morita, S., and Tawara, H. (1976). Bremsstrahlung induced by proton and He 3 -ion bombardments in the 1-4-MeV/amu energy range. Physical Review A, 13(1):131-138.

Ishii, K., Satoh, T., Matsuyama, S., Yamazaki, H., Tokai, Y., Sugimoto, A., and Yamamoto, K. (1999). Observation of Interference Between Atomic Bremsstrahlung and Nuclear Bremsstrahlung. International fournal of PIXE, 09(01n02):1-10.

Ishii, K., Yamazaki, H., Matsuyama, S., Galster, W., Satoh, T., and Budnar, M. (2005). Contribution of atomic bremsstrahlung in PIXE spectra and screening effect in atomic bremsstrahlung. X-Ray Spectrometry, 34(4):363-365. 
Jackson, J. D. (1999). Classical Electrodynamics. Wiley.

Jakubaßa-Amundsen, D. H. and Kleber, M. (1975). Bremsstrahlung in heavy-ion reactions. Zeitschrift für Physik A, 273(1):29-35.

Jeynes, C., Webb, R. P., and Lohstroh, A. (2011). Ion Beam Analysis: A Century of Exploiting the Electronic and Nuclear Structure of the Atom for Materials Characterisation. Reviews of Accelerator Science and Technology, 04(01):41-82.

Johansson, S. A. E., Campbell, J. L., and Malmqvist, K. G. (1995). Particle-induced X-ray emission spectrometry (PIXE). Wiley.

Kahaner, D., Moler, C., and Nash, S. (1989). Numerical methods and software. Prentice Hall.

Kissel, L., MacCallum, C., and Pratt, R. H. (1981). Bremsstrahlung energy spectra from electrons of kinetic energy $1 \mathrm{keV} \leq \mathrm{T} \leq 2000 \mathrm{keV}$ incident on neutral atoms $1 \leq \mathrm{Z} \leq 92$.

Kissel, L., Quarles, C., and Pratt, R. H. (1983). Shape functions for atomic-field bremsstrahlung from electrons of kinetic energy $1-500 \mathrm{keV}$ on selected neutral atoms $1 \leq \mathrm{Z} \leq 92$. Atomic Data and Nuclear Data Tables, 28(3):381-460.

Knoll, G. F. (2000). Radiation detection and measurement. Wiley.

Leo, W. R. (1994). Techniques for nuclear and particle physics experiments : a how-to approach. Springer.

Liberman, D., Waber, J. T., and Cromer, D. T. (1965). Self-Consistent-Field Dirac-Slater Wave Functions for Atoms and Ions. I. Comparison with Previous Calculations. Physical Review, 137(1A):A27-A34.

Mangiarotti, A. and Jakubaßa-Amundsen, D. H. (2017). Higher-order theories for relativistic electron-atom bremsstrahlung in comparison with experiment. Physical Review A, 96(4):042701.

Mangiarotti, A. and Martins, M. N. (2017). A review of electron-nucleus bremsstrahlung cross sections between 1 and $10 \mathrm{MeV}$. Radiation Physics and Chemistry, 141(May):312-338.

Mukoyama, T. (2015). M-shell ionization cross sections by proton impact on gold in the binaryencounter approximation. Nuclear Instruments and Methods in Physics Research, Section B: Beam Interactions with Materials and Atoms, 354:155-158. 
Mukoyama, T. (2017). M-X-ray production cross sections of heavy elements by charged-particle impact. Nuclear Instruments and Methods in Physics Research, Section B: Beam Interactions with Materials and Atoms, 408:182-186.

Mukoyama, T. (2018). N-shell ionization cross sections by proton impact in the BEA. X-Ray Spectrometry, 47(4):320-326.

Pia, M. G., Weidenspointner, G., Augelli, M., Quintieri, L., Saracco, P., Sudhakar, M., and Zoglauer, A. (2009). PIXE Simulation With Geant4. IEEE Transactions on Nuclear Science, 56(6):3614-3649.

Piessens, R., Doncker-Kapenga, E., Uberhuber, C. W., and Kahaner, D. K. (1983). Quadpack : a Subroutine Package for Automatic Integration. Springer Berlin Heidelberg.

Pratt, R. H., Tseng, H. K., Lee, C. M., Kissel, L., MacCallum, C., and Riley, M. (1977). Bremsstrahlung energy spectra from electrons of kinetic energy $1 \mathrm{keV} \leq \mathrm{T} 1 \leq 2000 \mathrm{keV}$ incident on neutral atoms $2 \leq \mathrm{Z} \leq 92$. Atomic Data and Nuclear Data Tables, 20(2):175-209.

Purcell, E. M. and Morin, D. J. (2013). Electricity and Magnetism. Cambridge University Press, 3 edition.

Reed, S. J. B. and Ware, N. G. (1972). Escape peaks and internal fluorescence in X-ray spectra recorded with lithium drifted silicon detectors. Fournal of Physics E: Scientific Instruments, 5(6):582-583.

Rehren, T., Belgya, T., Jambon, A., Káli, G., Kasztovszky, Z., Kis, Z., Kovács, I., Maróti, B., Martinón-Torres, M., Miniaci, G., Pigott, V. C., Radivojević, M., Rosta, L., Szentmiklósi, L., and Szőkefalvi-Nagy, Z. (2013). 5,000 years old Egyptian iron beads made from hammered meteoritic iron. Journal of Archaeological Science, 40(12):4785-4792.

Salvat, F. and Fernández-Varea, J. M. (2019). RADIAL: A Fortran subroutine package for the solution of the radial Schrödinger and Dirac wave equations. Computer Physics Communications, 240:165-177.

Scherzer, O. (1932). Über die Ausstrahlung bei der Bremsung von Protonen und schnellen Elektronen. Annalen der Physik, 405(2):137-160.

Schnopper, H. W., Delvaille, J. P., Kalata, K., Sohval, A. R., Abdulwahab, M., Jones, K. W., and Wegner, H. E. (1974). X-Ray Measurements Of Bremsstrahlung And Electron Capture By Fast Positive Ions. Physics Letters A, 47(1). 
Schoonjans, T., Brunetti, A., Golosio, B., Sanchez del Rio, M., Solé, V. A., Ferrero, C., and Vincze, L. (2011). The xraylib library for X-ray-matter interactions. Recent developments. Spectrochimica Acta Part B: Atomic Spectroscopy, 66(11-12):776-784.

Schoonjans, T., Brunetti, A., Golosio, B., Sanchez del Rio, M., Solé, V. A., Ferrero, C., and Vincze, L. (2015). https://github.com/tschoonj/xraylib.

Segui, S., Dingfelder, M., Fernández-Varea, J. M., and Salvat, F. (2002). The structure of the Bethe ridge. Relativistic Born and impulse approximations. Fournal of Physics B: Atomic, Molecular and Optical Physics, 35(1):33-53.

Sommerfeld, A. (1931). Über die Beugung und Bremsung der Elektronen. Annalen der Physik, 403(3):257-330.

Tseng, H. K. and Pratt, R. H. (1971). Exact Screened Calculations of Atomic-Field Bremsstrahlung. Physical Review A, 3(1):100-115.

Virtanen, P., Gommers, R., Oliphant, T. E., Haberland, M., Reddy, T., Cournapeau, D., Burovski, E., Peterson, P., Weckesser, W., Bright, J., van der Walt, S. J., Brett, M., Wilson, J., Millman, K. J., Mayorov, N., Nelson, A. R. J., Jones, E., Kern, R., Larson, E., Carey, C. J., Polat, I., Feng, Y., Moore, E. W., VanderPlas, J., Laxalde, D., Perktold, J., Cimrman, R., Henriksen, I., Quintero, E. A., Harris, C. R., Archibald, A. M., Ribeiro, A. H., Pedregosa, F., and van Mulbregt, P. (2020). SciPy 1.0: fundamental algorithms for scientific computing in Python. Nature Methods, pages $1-12$.

Wielopolski, L. and Gardner, R. P. (1976). Prediction of the pulse-height spectral distortion caused by the peak pile-up effect. Nuclear Instruments and Methods, 133(2):303-309.

Wielopolski, L. and Gardner, R. P. (1977). A generalized method for correcting pulse-height spectra for the peak pile-up effect due to double sum pulses: Part II. The inverse calculation for obtaining true from observed spectra. Nuclear Instruments and Methods, 140(2):297-303.

Yamadera, A., Ishii, K., Sera, K., Sebata, M., and Morita, S. (1981). Quasifree-electron bremsstrahlung induced by the projectile field. Physical Review A, 231.

Yuan, L. C. L. and Wu, C.-S. (1961). Nuclear Physics - Methods of Experimental Physics - Volume 5 Part A. Academic Press, New York. 